Open Access

OBM Geriatrics

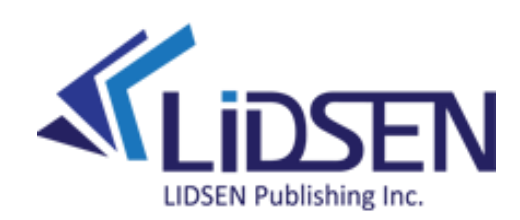

\section{OBM Geriatrics 2019}

\section{Volume 3, Issue 1}

Editor-in-Chief Professor Michael Fossel Printed Edition Published in OBM Geriatrics 


\section{Editorial Office}

73 Hongkong Middle Road, Qingdao, China

Tel./Fax: +86-532-8979-9572

E-Mail: geriatrics@lidsen.com

http://www.lidsen.com/journals/geriatrics

LIDSEN Publishing Inc.

2000 Auburn Drive, One Chagrin Highlands, Suite 200,

Beachwood, OH 44122, USA

Tel.: +1-216-370-7293

Fax: +1-216-378-7505

https://www.lidsen.com

This is a reprint of articles from the Issue 1 published online in the open access journal $O B M$ Geriatrics (ISSN 2638-1311) from January 01, 2019 to March 31, 2019.

Available at: http://lidsen.com/journals/geriatrics/geriatrics-issue-03-01

For citation purposes, cite each article independently as indicated on the article page online and as indicated below:

LastName, AA, LastName, BB, LastName, CC. Article Title. Journal Name Year;

Volume(Issue):Article Number; doi.

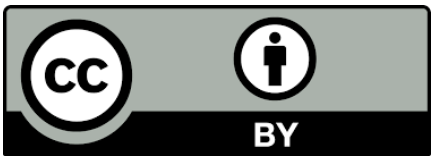

(c) 2019 by the authors. This is an open access article distributed under the conditions of the Creative Commons by Attribution License, which permits unrestricted use, distribution, and reproduction in any medium or format, provided the original work is correctly cited. 


\section{Contents}

\section{Ana Jessica Alfaro, Soledad Argüelles-Borge, Ashley M. Stripling, Paula M. Brochu}

The Positive Power of Perception in Late Life: A Study on Subjective Aging and Health among Ethnic Minorities

Reprinted from: OBM Geriatrics 2019;3(1):16; doi:10.21926/obm.geriatr.1901043

\section{Ray Marks}

Depression and Aging: Role of Social Support

Reprinted from: OBM Geriatrics 2019;3(1):4; doi:10.21926/obm.geriatr.1901042

Lei Zhang, Xian-Zhang Hu, Xiaoxia Li, Jacob Dohl, Tianzheng Yu, Robert J. Ursano

Association of Telomere Length, a Cellular Aging Marker, with Depression, PTSD and Hostility

Reprinted from: OBM Geriatrics 2019;3(1):14; doi:10.21926/obm.geriatr.1901041

\section{Robert J Emanuel}

Does Dementia Increase the Risk of Dental Decay?

Reprinted from: OBM Geriatrics 2019;3(1):10; doi:10.21926/obm.geriatr.1901040 35

Robert L. Conder, Jr., Christopher Friesen, Alanna A. Conder

Behavioural and Complementary Interventions for Healthy Neurocognitive Aging

Reprinted from: OBM Geriatrics 2019;3(1):15; doi:10.21926/obm.geriatr.1901039

\section{Ali Al-Ragawi, Sergey Zyryanov, Elena Ushkalova, Olga Butranova, Anton Pereverzev}

Prediction of ADRs and Estimation of Polypharmacy in Older Patient's Population: Retrospective Study in Russian Gerontology Center

Reprinted from: OBM Geriatrics 2019;3(1):13; doi:10.21926/obm.geriatr.1901038 60

Kristopher M. Struckmeyer, Alex J. Bishop, Brenda J. Smith, Brandt C. Gardner

Exploring the Paradox of Care: Differences in Older Adult and Proxy Ratings of Health and Subjective Well-Being

Reprinted from: OBM Geriatrics 2019;3(1):19; doi:10.21926/obm.geriatr.1901037 73

Vincenza Frisardi, Sara Faroni, Angela Bellani, Felice Biagi, Emanuela Galante, Alida Balzanelli, Erika Talassi, Lorella Frittoli, Giancarla Capiluppi, Claudia D’angelis, Antonella Taragnani, Donatella Terzi, Elena Podavini, Graziana Gazzoni, Graziana Simoncelli, Carmine Matarazzo, Mirko Avesani, Antonio Ventura, Consuelo Basili, Maurizio Galavotti, Carlo Maria Stucchi, Maria Cristina Cilia, Alfonso Ciccone

Improving the HealthCare of People with Dementia beyond the Diagnosis: The "Carlo Poma Dementia Care Pathway" Study Protocol

Reprinted from: OBM Geriatrics 2019;3(1):18; doi:10.21926/obm.geriatr.1901036 92 


\section{Steven R. Brenner}

Sporadic Alzheimer Disease and That Developing in Down's Syndrome: The Immune System Attacking Self Rather Than Suppressing Infectious Disease Invaders, Toll Like Receptors Triggering Excessive Cytosolic Calcium, Excess Calcineurin Activation, Overexpression of Regulator of Calcineurin1, Runaway Beta-Amyloid Production, Synaptic Loss, Destructive Inflammation and Dementia Reprinted from: OBM Geriatrics 2019;3(1):9; doi:10.21926/obm.geriatr.1901035 110

\section{Michael Fossel}

Cell Senescence, Telomerase, and Senolytic Therapy

Reprinted from: OBM Geriatrics 2019;3(1):14; doi:10.21926/obm.geriatr.1901034

\section{Marco Barale, Ruth Rossetto Giaccherino, Ezio Ghigo, Massimo Procopio}

Effect of Six-Months Supplementation with Cholecalciferol on Glycemic and Blood Pressure Control in Elderly Type 2 Diabetic Patients with Vitamin D Deficiency: A Pilot Study

Reprinted from: OBM Geriatrics 2019;3(1):15; doi:10.21926/obm.geriatr.1901033 133

\section{Rachel Mills, Louisa Jackman, Mithila Mahesh, Ian James}

Key Dimensions of Therapeutic Lies in Dementia Care: A New Taxonomy

Reprinted from: OBM Geriatrics 2019;3(1):12; doi:10.21926/obm.geriatr.1901032 148

\section{Michael Fossel}

Telomere Editorial - Perspectives on Telomeres and Aging

Reprinted from: OBM Geriatrics 2019;3(1):7; doi:10.21926/obm.geriatr.1901031 160

\section{Anya Ahmed, Paula Ormandy, Maaike L. Seekles}

An Examination of How the 'Household Model' of Care Can Contribute to Positive Ageing for Residents in the 'Fourth Age'

Reprinted from: OBM Geriatrics 2019;3(1):24; doi:10.21926/obm.geriatr.1901030 167

Elizabeth Orsega-Smith, Stephen Goodwin, Melissa Ziegler, Katie Greenawalt, Jennie Turner, Erica Rathie

Aging and the Art of Happiness: Time Effects of A Positive Psychology Program with Older Adults Reprinted from: OBM Geriatrics 2019;3(1):19; doi:10.21926/obm.geriatr.1901029

\section{Filomena Papa, Bartolomeo Sapio, Enrico Nicolò}

Acceptance of Information and Communication Technologies for Healthy and Active Aging: Results from Three Field Studies

Reprinted from: OBM Geriatrics 2019;3(1):22; doi: 10.21926/obm.geriatr.1901028. 210 
Original Research

\title{
The Positive Power of Perception in Late Life: A Study on Subjective Aging and Health among Ethnic Minorities
}

\author{
Ana Jessica Alfaro, Soledad Argüelles-Borge, Ashley M. Stripling ${ }^{*}$, Paula M. Brochu
}

Nova Southeastern University, 3301 College Avenue, Fort Lauderdale, Florida, USA; E-Mails: aa2106@mynsu.nova.edu, soledada@nova.edu, astripling@nova.edu, pbrochu@nova.edu

* Correspondence: Ashley M. Stripling; E-Mail: astripling@nova.edu

Academic Editor: Lisa Hollis-Sawyer

Special Issue: Got Aging? Examining Later-life Development from a Positive Aging Perspective

OBM Geriatrics

2019, volume 3, issue 1

doi:10.21926/obm.geriatr.1901043
Received: September 17, 2018

Accepted: March 25, 2019

Published: March 29, 2019

\begin{abstract}
Background: The world's aging population is growing and diversifying at an unprecedented rate. In preparation for this, the National Institutes of Health and the Centers for Disease Control and Prevention have issued calls to improve the overall health of older adults. A promising avenue of research to improve health in late life has been dedicated to subjective aging; the way people perceive their own aging process. The main objective of this empirical study was to investigate subjective aging in late life among ethnic minorities in the United States. Specifically, this socio-historic study examined age identity, or how old one feels, in relation to psychological well-being and functional health.
\end{abstract}

Methods: This study utilized data from the Midlife Development in the United States Study (MIDUS), Survey of Minority Groups.

Results: Results indicated the sampled population predominately reported a younger age identity, that is, felt younger than their actual age with higher age related to great discrepancy between felt and actual age. Although there were no differences in aging identity among specific ethnic backgrounds or levels of education, gender-related differences were found with men feeling younger than women. Another key finding was that younger age identity predicted higher levels of psychological well-being and functional

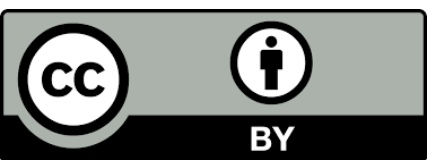

(C) 2019 by the author. This is an open access article distributed under the conditions of the Creative Commons by Attribution License, which permits unrestricted use, distribution, and reproduction in any medium or format, provided the original work is correctly cited. 
health. Lastly, psychological well-being mediated the association between age identity and functional health, even while controlling for age and gender.

Conclusions: These findings contribute to the literature by extending previous research on subjective aging and health to ethnic minorities. Moreover, these results have clinical and research implications for professionals in the field of geriatrics.

\section{Keywords}

Subjective aging; gerodiversity; positive aging; successful aging; psychological well-being

\section{Introduction}

The world's aging population is growing at an unprecedented rate. In 2010, 8 percent of the world's population was aged 65 or older [1]. By 2050, it is expected to nearly double to 16 percent [1]. In a like manner, the United States Census Bureau reported that in 2030 one in every five Americans is projected to be retirement age [2]. By 2060, older adults are predictable to make up nearly one-quarter of the population [2]. Largely caused by the aging of the baby boomers, this rapid growth will inevitably create a demographic shift in our nation. In preparation for this, the National Institutes of Health (NIH) and the Center for Disease Control and Prevention (CDC) issued a call for action to improve the overall health of older adults living in the United States [3]. In order to prepare for this foreseeable demand in geriatric care, it is important to take into account the ethnic and cultural diversity of our nation's aging population. The growth of older Americans defined as "minority" by the federal government (including African American, Hispanic or Latino, American Indian and Alaska Native, and Asian and Pacific Islanders) is projected to grow more rapidly than the non-Hispanic white population of older Americans throughout the next half century [4]. By mid-century, one-third of older Americans are projected to be from a minority population [5]. Consequently, the U.S. health care system has coined the term "ethnogeriatric imperative" to foreshadow the challenges of serving the fast-growing segment of ethnic minorities in late life [4]. As the United States continues to diversify, a commitment to cultural context needs to be more explicitly included in gerontological theories and research.

\subsection{Subjective Aging}

A promising avenue of research to improve health in late life has been dedicated to subjective aging, the way people perceive their own aging process [6]. According to Ryff, experiences of subjective aging and a person's awareness of aging are integral psychological processes of the aging self [7]. How a person understands and attributes meaning to their own aging process is a specific form of self-knowledge that may contribute to how individuals prevent and cope with illness in later life $[8,9]$. More compelling evidence revealed that subjective aging is better at predicting psychological and physical functioning than one's actual age [10-13]. Given these points, successful aging is largely subjective, and is a concept that has sparked empirical interest in the fields of social gerontology, life span psychology, and life course sociology [8]. 
Subjective aging is a broad concept that has been conceptualized in several different ways in the research literature. Two terms commonly used are self-perceptions of aging and age identity [9]. Self-perceptions of aging refer to a general evaluation of how aging experiences are interpreted, and is often associated with satisfaction towards aging [9, 13]. More specifically, age identity ( $\mathrm{Al}$ ) numerically captures the difference between one's chronological age and one's felt age, and is traditionally assessed by asking a person "how old do you feel?" $[10,14]$. Overall, the subjective aging literature has postulated that feeling younger and having positive representations of one's own aging process are both associated with better physical and psychological functioning in aging individuals $[8,9]$.

Although it is undisputable that the most objective measure of aging is age itself, the cultural maxim "age is nothing but a number" reveals a societal consensus that downplays the significance of a person's chronological age. Several lines of research support this consensus by showing that across the lifespan, the discrepancy between subjective and chronological age accelerates $[15,16]$. For example, a cross-sectional Danish study found that younger respondents felt older and older respondents felt younger [17]. Respondents 40 years and older reported they felt younger (roughly $20 \%$ younger) than their actual age [18]. This trend was also seen in the Berlin Aging study, where 6-year longitudinal data revealed that on average, adults aged 70 years and older felt about 13 years younger than their actual age over time [19]. In short, the ability to maintain positive self-perceptions of aging, such as feeling young, appears to be central to psychosocial adjustment throughout life $[17,20,21]$.

Subjective aging is thought to be shaped by both individual and sociocultural factors $[6,9]$. Based on the classical theories about the self and identity, two self-motivating processes may be at work in shaping age identities: Self-consistency, the motive to remain the same stable person, and self-enhancement, the motive to maintain a positive self-image over time [7, 22]. From this developmental perspective, feeling younger than one's actual age is the result of an assimilation process that maintains an existing identity. According to Westerhof and Wurm, "individuals are able to maintain consistency in late life by integrating new experiences into their existing selfconcepts and thereby identifying with the younger age they used to be" [9] (pg. 148). In youthcentered cultures like the United States, aging is seen as threating to an individuals' self-concept, therefore identifying with younger age groups can help maintain a consistent and positive selfconcept $[21,23]$. Sneed and Whitbourne deemed this as an assimilative strategy in terms of lifespan identity processes [24].

In a similar fashion, the discrepancy in age and felt age in late life can be understood as a selfenhancing strategy through the lens of age-denial theory [16]. This view postulated that denial of aging is a defense by which aging adults can dissociate themselves from the stigma related to growing old [16]. Living in an ageist environment can have debilitating effects on older adults as evidence by Levy who found that older adults who were primed with negative stereotypes of old age performed worse on memory tasks, handwriting, and walking compared to those primed with positive stereotypes [17]. Therefore, resisting the internalization of ageist stereotypes by feeling younger can compensate for the negative implications of ageism [25]. In spite of the differences in terminology, these theoretical explanations converge on the premise that subjective aging is an adaptive strategy in growing old [6].

On the sociocultural level, mass media, social policies, societal institutions, and cultural values are considered [9]. A tendency to report a youthful subjective age is more common in Western 
societies where youth is celebrated and growing old is stigmatized [20, 26]. For example, two studies that compared the United States to Finland and to Japan found that the discrepancy between felt age and chronological age was larger among Americans [27, 28]. Similarly, Westerhof, Barrett, and Steverink found that compared to Germans, Americans maintained more youthful identities, particularly at older ages [29]. Moreover, a cross-cultural study of Americans and Dutch persons aged 40 to 85 revealed that the self-enhancing function of youthful and positive aging perceptions was stronger in America than in the Netherlands [30]. Building on these empirical findings, one can conclude that positive perceptions of aging have a stronger impact and higher adaptive value in cultures that favor youthfulness to a greater degree like in Western societies.

In summary, the way individuals perceive their aging is an intricate process involving personal experiences, social interactions, cultural values, and societal structures [31]. Therefore, it is prudent to take individual and sociocultural contexts into account when examining subjective aging. Moreover, feeling younger in late life is considered a strategy used to adapt to age-related changes by maintaining a positive sense of identity and by distancing oneself from the stigma related to growing old, especially in cultures that devalue old age [24, 29, 32]. The effectiveness of this strategy has been empirically supported by studies finding that feeling younger than one's chronological age is related to well-being and health over time [6, 10, 32-34].

\subsection{Subjective Aging and Health}

Since the appearance of subjective aging in the literature, research has aimed to conceptualize, identify, and understand the pathways through which it is related to health. Westerhof and Wurm brought forth a heuristic model linking subjective aging, psychological resources, health, and survival within the individual and sociocultural context [9]. Used to guide further research, this model hypothesized that subjective aging contributes to the accumulation of psychological resources, which in turn helps maintain good health [9]. Today, accruing evidence supports this model by demonstrating that feeling younger than one's age is associated with well-being and a range of positive health-related outcomes.

To begin with, age identity has been demonstrated to be associated with facets of psychological well-being (PWB). Nearly 25 years ago, Ryff put forth a model of psychological wellbeing that offered a notable contrast in scientific research on well-being [7]. The Ryff model introduced an eudaimonic approach to well-being, which in turn, centered around finding meaning, self-realization, and experiences of optimal growth [35, 36]. The development of this integrated model of well-being drew on the union of life-span developmental theories, clinical theories of personal growth, and mental health perspectives [37]. This model has been widely used by other investigations, to study well-being in response to various life challenges such as health changes of later life [35, 38]. In fact, older age identity, that is feeling older than one's actual age, has been associated with lower psychological well-being and higher levels of negative affect among middle-aged and older adults [14]. Whereas younger age identity, feeling younger than one's age is related to higher levels of life satisfaction, positive affect, lower risk of major depressive episode and higher chance of flourishing mental health [21, 32, 39].

In addition to having an effect on psychological well-being, subjective aging has also been found to impact older adult's ability to perform activities of daily life. For instance, an experimental study of older adults induced a younger subjective age by providing positive 
feedback following their performance on a hand grip task [40]. Results revealed that those who received feedback exhibited improved performance on the second-hand grip task as opposed to those who did not receive feedback [40]. This study demonstrated that inducing a younger subjective age can promote physical functioning, a direct implication for improving functional health in late life.

The 2008 Physical Activity Guidelines for Americans published by the U.S. Department of Health and Human Services (HHS) defined functional ability as "the capacity of a person to perform tasks or behaviors that enable him or her to carry out everyday activities" [41] (pg.13). Examples of functional health include ability to walk up a flight of stairs or carry groceries, and a compilation of studies have found that having positive perceptions of aging can promote these abilities. For example, Sargent-Cox, Anstey and Luszcz found that positive self-perceptions of aging had an effect on objective physical performance tests such as balance, gait, and rising from a chair over a period of 16 years in adults aged 65 and older [13]. In a similar fashion, Levy, Slade, and Kasl found that adults aged 50 years and older with more positive self-perceptions of aging reported better functional health, such as ability to walk up and down stairs, than those with more negative selfperceptions of aging over an 18-year period [42]. In fact, this effect was significant even after controlling for baseline measures of functional health, self-rated health, age, gender, race, and socioeconomic status [42]. Furthermore, the Midlife in the United States (MIDUS) national study of health and well-being has also shown interest in this area of research as evident by the release of a newsletter titled "Subjective Aging". This publication reported that Caucasians who felt older than their age reported more difficulties with basic activities, such as bathing and dressing as well as more strenuous activities such as carrying groceries and climbing several flights of stairs compared to those who felt younger than their age [43].

In conclusion, the notion that positive self-perceptions of aging, such as feeling younger than one's age, is beneficial to one's psychological well-being and functional health in late life is well established in the literature; this association holds across diverse study samples and across different countries and cultures [10, 21, 44]. However, to the authors' knowledge, there remains a paucity of published research that examines age identity among older adults of ethnic minority backgrounds in America. Therefore, to effectively meet the challenges of a rapidly growing segment of our population, better understanding of how this group experiences aging within the context of American culture is warranted.

\subsection{Present Study}

In order to address this paucity of ethnogeriatric research examining perception of aging among ethnic minorities in late-life in the context of a youth-centered culture, this current study utilized a socio-historic paradigm explored subjective aging, specifically age identity, in late life among African Americans, Puerto Ricans, Dominicans, and Mexicans in the United States. On the basis of the literature, it was hypothesized that ethnic minorities in late life felt younger than their chronological age. Given that all respondents live within the same cultural context, differences in age identity were not expected between ethnicities. Second, this study examined PWB and functional health in relation to age and age identity. A positive association between PWB and functional health was hypothesized. It was also hypothesized that respondents would feel younger the older they were. Moreover, it was predicted that feeling younger than one's age would be 
associated with higher levels of PWB and functional health and that PWB would mediate the association between age identity and functional health. This study fills a gap in the literature by contributing to understanding the influence of age identity on psychological well-being and functional health, particularly among a sample that has been neglected in the field of subjective aging.

\section{Materials and Methods}

\subsection{Participants and Procedure}

This empirical study examined cases from the Midlife in the United States (MIDUS), Survey of Minority Groups. The survey was part of a larger project that aimed to investigate the patterns, predictors, and consequences of midlife development in the United States within the areas of physical health, psychological well-being, and social responsibility [45]. In particular, the minority survey assessed the well-being of urban, ethnic minority adults who lived in Chicago and New York. The data was collected from 1995 to 1996 through structured interviews and included minority residents 25 years or older living in both hyper-segregated neighborhoods and in areas with lower concentrations of minorities.

The data used for this socio-historic study is archival and a subset of the entire sample that was collected. Given the focus on late life, the sample only included respondents from ages 55 to 99 ( $N$ $=268, M=64.79, S D=7.957)$. Of the respondents, $54.9 \%$ were men and $45.1 \%$ were women. The ethnic minority groups represented were African Americans (34.7\%), Puerto Ricans (39.2\%), Dominicans (16.4\%), and Mexicans (9.7\%). Out of the 255 respondents who answered about their highest level of education, $25.7 \%$ of the sample reported some grade school education, $10.8 \%$ received anywhere between $6^{\text {th }}$ and $8^{\text {th }}$ grade education, $39.6 \%$ had high school education, and $19 \%$ having reported college or graduate level education.

The current study utilized secondary MIDUS data collection which is reviewed and approved by the Education and Social/Behavioral Sciences and the Health Sciences IRBs at the University of Wisconsin-Madison. For additional information please refer to the MIDUS website at https://www.icpsr.umich.edu/icpsrweb/ICPSR/studies/2856/summary.

\subsection{Measures}

\subsubsection{Subjective Aging}

Subjective aging has been conceptualized several different ways in the research literature [9]. In the context of this study, subjective aging is measured using age identity, or the difference between one's subjective (felt or perceived) age and one's chronological age [29]. One's subjective age is usually measured with a single-item such as "What age do you feel?" [46]. The participants in this study were asked "Many people feel older or younger than they actually are. What age do you feel most of the time?" The chronological age was obtained by examining date of birth. A variable was computed to capture the difference between participants' chronological age and the age they feel most of the time. If the result yielded a positive value, then the participant felt younger than their chronological age, thus reporting a younger age identity. Conversely, yielding a 
negative value is interpreted as endorsing an older age identity, where a participant felt older than their chronological age.

\subsubsection{Psychological Well-Being}

Psychological well-being was measured using Ryff's theoretical framework, an approach that has been used within other studies of social factors and psychological well-being [47-49]. Ryff proposes six dimensions to measure psychological well-being: autonomy, environmental mastery, personal growth, positive relations with others, purpose in life, and self-acceptance. Each domain was assessed on a 7-point Likert scale using 3 items per domain, for a total of 18 items. Items were reverse coded as needed and scores were summed to form a single composite measure of psychological well-being, where higher scores represented greater levels of psychological wellbeing.

\subsubsection{Functional Health}

Functional health, or one's ability to perform activities of daily living, was measured averaging the seven-item Physical Functioning subscale of the SF-36 Health Survey, with higher scores indicating better functional health [50]. The items related to the degree to which the participants' health limits them in certain activities (e.g., lifting or carrying groceries, bathing or dressing, climbing flights of stairs).

\subsubsection{Sociodemographic Variables}

The structured interviews included basic demographic questions relating to age, gender, race/ethnicity, and education. Age was examined as a continuous variable. Gender was coded 1 for men and 2 for women. Race was coded into the four ethnicities represented: African Americans, Puerto Ricans, Dominicans, and Mexicans. Highest level of education was coded using four categories: Some grade school education, anywhere between $6^{\text {th }}$ and $8^{\text {th }}$ grade education, high school education, and college or graduate level education.

\section{Results}

The main objective of this empirical study was to investigate the effects of subjective aging on health in late life among four ethnic minority groups living in the United States. The aims were trifold: (1) To examine age identity; (2) to explore the association between age, age identity, psychological well-being, and functional health; and (3) to investigate whether the association between age identity and functional health is mediated by psychological well-being. Each of the study's specific aims and their associated results are presented below. Descriptive statistics for these variables are available in Table 1. 
Table 1 Descriptive statistics for overall sample $(N=268)$.

\begin{tabular}{lllll}
\hline Measures & $M$ & SD & Min & Max \\
\hline 1. Age & 64.79 & 7.957 & 55 & 99 \\
2. Age Identity & 14.9328 & 15.85831 & -28 & 69 \\
3. PWB & 99.8358 & 14.68352 & 63 & 125 \\
4. Functional Health & 3.6067 & 0.37387 & 3 & 4 \\
\hline
\end{tabular}

\subsection{Age Identity}

\subsubsection{Research Question 1a}

Do late-life African Americans, Puerto Ricans, Dominicans, and Mexicans who participated in the Midlife in the United States (MIDUS) Survey feel younger than their chronological age?

Age identity was calculated by finding the difference between the respondents' chronological age and the age they felt most of the time. Following the computation of age identity, frequency data revealed that on average, participants reported a younger age identity, that is, felt younger than their chronological age $(M=14.9, S D=15.85)$.

\subsubsection{Research Question 1b}

Does age identity of late life African Americans, Puerto Ricans, Dominicans, and Mexicans who participated in the Midlife in the United States (MIDUS) Survey differ as a function of ethnicity, level of education, and gender?

A one-way between-subjects ANOVA was conducted to examine the effect of ethnicity on age identity among Puerto Ricans $(M=15.57, S D=17.31)$, Dominicans $(M=15.14, S D=13.54)$, African Americans $(M=15.04, S D=15.42)$, and Mexicans $(M=11.61, S D=15.33)$. No significant associations were observed between age identity and ethnicity or education. However, a significant difference in age identity was found between men $(M=16.67, S D=15.08)$ and women $(M=12.82, S D=16.6) ; t(266)=1.99, p=.047$.

\subsection{Association between Age Identity, Psychological Well-Being and Functional Health}

\subsubsection{Research Question 2a}

Are age and age identity related to psychological well-being and functional health?

Bivariate correlations were conducted to examine the association between chronological age, age identity, PWB, and functional health. As expected, age and age identity were closely related, $r(268)=.332, p<.01$, demonstrating that the older an individual, the higher the discrepancy between chronological and felt age. In addition, there was a positive correlation between PWB and functional health $r(268)=.155, p<.05$. Younger age identity was associated with higher levels of psychological well-being, $r(268)=.180, p<.01$ and functional health, $r(268)=.246, p<.01$. However, chronological age was not statistically associated with PWB nor functional health (see Table 2). 
Table 2 Correlations between measures for overall sample $(N=268)$.

\begin{tabular}{lllll}
\hline Measures & $\mathbf{1}$ & $\mathbf{2}$ & $\mathbf{3}$ & $\mathbf{4}$ \\
\hline 1. Age & -- & & & \\
2. Age Identity & $.332^{* *}$ & -- & & \\
3. PWB & .102 & $.180^{* *}$ & -- & \\
4. Functional Health & -.062 & $.246^{* *}$ & $.155^{*}$ & -- \\
\hline \multicolumn{5}{c}{$* p<.05 .^{* *} p<.01}$.
\end{tabular}

\subsubsection{Research Question 2b}

Are age, age identity, and gender predictive of PWB and functional health?

Two linear regressions were conducted to predict psychological well-being and functional health based on age, age identity, and gender to examine the predictive utility of age identity above and beyond these other predictors. Age identity significantly predicted psychological wellbeing $(\beta=.14, S E=.06, p=.019)$, whereas age and gender did not. The second regression analysis found that age $(\beta=-.01, S E=.003, p=.013)$, gender $(\beta=-.12, S E=.04, p=.007)$, and age identity $(\beta=.01, S E=.001, p<.001$ ) were all significantly predictive of functional health (see Table 3 and Table 4).

Table 3 Regression coefficients predicting functional health for $(n=268)$.

\begin{tabular}{lll}
\hline & \multicolumn{2}{l}{ Functional Health } \\
\cline { 2 - 3 } & $\beta$ & $p$ \\
\hline Age & -.007 & .013 \\
Gender & -.120 & .007 \\
Age Identity & .007 & .000 \\
\hline
\end{tabular}

Table 4 Regression coefficients predicting psychological well-being for $(n=268)$.

\begin{tabular}{lll}
\hline & \multicolumn{2}{l}{ Functional Health } \\
\cline { 2 - 3 } & $\beta$ & $p$ \\
\hline Age & .096 & .416 \\
Gender & -2.762 & .124 \\
Age Identity & .140 & .019 \\
\hline
\end{tabular}

\subsection{Mediation}

\subsubsection{Research Question 3}

Does PWB mediate the association between age identity and functional health? 
A mediation analysis was conducted following the recommendations of Preacher and Hayes to investigate the hypothesis that psychological well-being mediated the association between age identity and functional health [51]. The mediation analysis controlled for gender and age as covariates. The analysis showed that age identity was significantly associated with psychological well-being, $\beta=.14, S E=.06, p=.019$, and that psychological well-being was marginally associated with functional health, $\beta=.003, S E=.002, p=.069$. Age identity was also significantly associated with functional health, $\beta=.01, S E=.001, p<.001$ (see Figure 1). Most importantly for the mediation analysis, bootstrap analyses revealed that the $95 \%$ confidence interval for the size of the indirect effects excluded zero $(\mathrm{Cl}=.00001, .0012)$, indicating mediation [50]. Thus, psychological well-being mediated the association between age identity on functional health. Approximately $12 \%$ of the variance in functional health was accounted for by the predictors $\left(R^{2}\right.$ $=.12$ ) (see Figure 1).

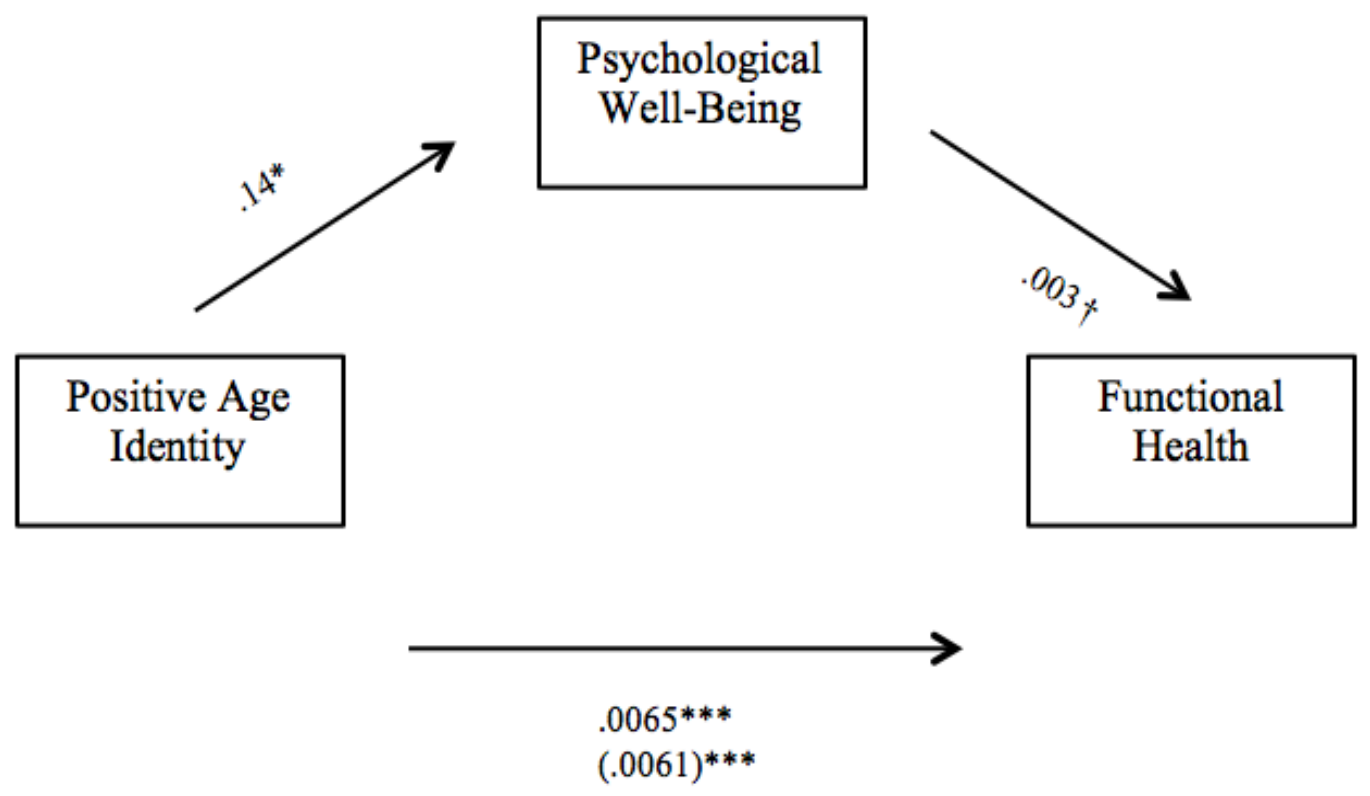

Note. Standardized betas are indicated on figure paths. Number in brackets represent beta when psychological well-being, was entered in the analysis. $R^{2}=.12$; adjusted $R^{2}=.11$.

${ }^{*} p<.05 .{ }^{* *} p<.01 .^{* * *} p<.001 . \dagger \mathrm{n}=.07$

Figure 1 Psychological well-being as a mediator of younger age identity on functional health.

\section{Discussion}

\subsection{Review of Study Findings}

The main purpose of this study was to explore age identity in late life among individuals from four ethnic minority groups in the United States, a neglected area in the subjective aging literature. The present study has six major findings. First, individuals aged 55 and older of African American, Puerto Rican, Dominican, and Mexican backgrounds felt younger than their actual age. However, 
when taking into account their specific ethnic background, no differences were found. Given the cultural context, this finding illustrated the adaptive value of feeling younger in America, a youthcentered society [21]. In addition, this study showed that getting older was associated with feeling younger. This is not surprising, considering the evidence that the discrepancy between felt and chronological age increases as individuals age [18,52]. Individual factors such as a motive to maintain a consistent and positive self-concept is an assimilative strategy that can help explain this discrepancy [24].

Second, the existence of gender-related differences in age identity is unresolved in the literature. Some studies have found that woman report greater discrepancies than men, whereas others have shown that men perceive themselves as younger more often than women $[16,53]$. To further emphasize the mixed results, some studies did not find any gender-related differences [18] Nevertheless, this study revealed that in late life, ethnic minority men felt younger than ethnic minority women. Culturally-specific factors may play a role in this finding and therefore merits future consideration. For example, machismo, a strong sense of masculinity that is related to a man's responsibility to protect and defend his family, could be considered [54]. Men with traditional gender identities tend to avoid situations in which they perceive themselves as helpless or weak, thereby the process of aging can result in a loss of machismo [55, 56]. As a result, feeling younger than their age can be understood as a way of coping that facilitates avoidance of agerelated loss in men of ethnic backgrounds [57].

Third, this study found that younger age identity was associated with higher levels of psychological well-being and functional health, whereas chronological age was not. This finding highlights the importance of age identity over chronological age in late life, which has direct implications for clinicians working with this population. In essence, simply asking a person's chronological age does not suffice. Assessing for an individual's perception of their own aging, such as by asking how old they feel, may reveal valuable information about the older adult's resilience and vitality if conducted in a manner that belies a dynamic understanding of the lived experiences of older adults. Another important point is that therapists should be cognizant of the way they communicate with this population to avoid engaging in ageist remarks that may influence the way their patients perceive their age.

Fourth, this study showed that age, gender, and age identity all predicted functional health, but only age identity predicted PWB. Age and gender are generally fixed; therefore, age identity seems to be the most feasible as a mean to promote functional health. Moreover, the strong predictive nature of age identity on both functional health and PWB demonstrated that one's perception of aging has a strong influence on successful aging. The fifth main finding revealed that psychological well-being also predicted functional health. This implies that promoting access to psychological services for older adults is an arena for advocacy. Moreover, these findings imply that solely treating physical ailments would be an injustice to the overall health of this population, and as a result, health professionals should be advised to refer their patients to seek psychological services when deemed necessary.

Lastly, as expected, PWB mediated the association between age identity and functional health. In short, the power of perception in late life is monumental in predicting facets of well-being and health. These results have numerous implications for clinicians working with this population and researchers interested in advancing the field of geriatrics. Ultimately, future intervention research 
on leveraging age identity and promoting positive perceptions of aging within individual and sociocultural contexts is warranted.

\subsection{Limitations and Future Research Directions}

Some limitations of the study should be noted. Self-report measures were used for all variables, and the associations between variables could be affected by a common measurement bias. Yet, psychosocial variables such as psychological well-being are typically measured using self-report, given the focus on perceptions and expectancies [58]. It is difficult, if not impossible, to include an objective measure of age identity because the nature of the construct is based solely on perception. However, future research can incorporate performance-based measures such as gait tasks to objectively assess functional health. Given that functional ability is only one area of health, future research is encouraged to explore the implications of younger age identity on other areas, such as physical health. Moreover, given the complexity of subjective aging, many argue that youthful age identity is only one aspect of the aging self. However, despite relying on a single-item measure, age identity ratings have shown considerable predictive validity and remain important in making strong empirical predictions in subjective aging research [8]. Future studies should examine other conceptualizations of subjective aging to establish stronger empirical foundation for the use of multidimensional versus unidimensional approaches and vice versa. In addition, regardless of the model or definitions used, most studies are based on researchers' conceptualizations and measures of successful aging and relatively few studies have addressed aging well from the perspective of older adults, especially those in minority groups. This is a concern, given the magnitude of projected population increases in ethnic minority groups in latelife by the mid-century.

Another limitation was the cross-sectional nature of the study and the use of historic data. While adding to greatly underexplored area, namely subjective aging in ethnic minorities in late life, the population assessed has likely already entered late life with many in the old-old category based on the latest census data. As such, future research should reexamine the population to better understand the nature of this relationship over time through longitudinal and intradindividual inquires thus increasing understanding of the variability and temporal relationship between age identity, functional health and psychological well-being. This would also allow for a current and more dynamic view of subjective aging. The issue of generalizability in terms of what the federal government considers "ethnic minorities" was another limitation. Lack of data collected on American Indians, Alaska natives, Asians, and Pacific Islanders prevents the findings to be considered inclusive of ethnic minorities in the United States [5]. Consequently, in order to prepare for the ethnogeriatrics imperative, examining subjective aging among these previously stated populations is a potential area of future research. Moreover, taking a closer look at culturespecific values that may influence successful aging, specifically age identity, lends itself to future avenues within the realm of subjective aging in ethnic minorities.

Lastly, this study did not take into consideration acculturation status, that is the process by which immigrants adapt psychologically and behaviorally to new sociocultural environments [59]. Based on the findings of this study, it is expected that subjective aging is less important for ethnic minorities from cultures that value the elderly, if they have not assimilated as strongly into US culture. In other words, ethnic minorities in late who have acculturated, may value youthfulness 
more than those who have recently arrived in this country or have assimilated to a lesser degree. In order to support this prediction, future research should aim at captivating the effects of acculturation status on the perceptions of aging and late life in ethnic minorities in the US.

\section{Conclusions}

Given the unprecedented rise in ethnic minorities reaching old age in the United States, new ways to promote physical and psychological well-being is warranted. Across multiple samples, cultures, and countries, studies have found that subjective aging is an important predictor of health and a viable psychological process used to adjust to challenges in late life. The current study adds to that body of research by showing that subjective age identity can influence levels of psychological well-being and functional health in ethnic minority older adults. As the research on subjective aging is limited in regard to the ethnic minority population in the United States, the hopes of the authors is that the current study guides future inquiry and general public health initiatives.

\section{Acknowledgments}

The authors would like to thank the participants who participated in the Midlife Development in the United States Study (MIDUS), Survey of Minority Groups.

\section{Author Contributions}

Dr. Argüelles-Borge contributed to the design and revisions of the manuscript. Dr. Stripling contributed to the revisions, format and submission of the manuscript. Dr. Brochu contributed to the revisions, design, and statistical data analysis presented in the manuscript.

\section{Competing Interests}

The authors have declared that no competing interests exist.

\section{References}

1. He W, Goodkind D, Kowal P. An aging world: 2015. Washington: U.S. Census Bureau; 2016.

2. Vespa J, Armstrong DM, Medina L. Demographic turning points for the United States: Population projections for 2020 to 2060. U S Census Bureau Curr Popul Rep. 2018; P25-1144.

3. Centers for Disease Control and Prevention. The state of aging and health in America. Atlanta: Centers for Disease Control and Prevention, US Department of Health and Human Services; 2013.

4. Yeo G. How will the US healthcare system meet the challenge of the ethnogeriatric imperative?. J Am Geriatr Soc. 2009; 57: 1278-1285.

5. Ortman JM, Velkoff VA, Hogan H. An aging nation: The older population in the United States. U S Census Bureau Curr Popul Rep. 2014; P25-1140.

6. Westerhof GJ, Miche M, Brothers AF, Barrett AE, Diehl M, Montepare JM, et al. The influence of subjective aging on health and longevity: A meta-analysis of longitudinal data. Psychol Aging. 2014; 29: 793-802. 
7. Ryff CD. Beyond Ponce de Leon and life satisfaction: New directions in quest of successful ageing. Int J Behav Develop. 1989; 12: 35-55.

8. Diehl $M$, Wahl $H$, Brothers $A$, Miche $M$. Subjective aging and awareness of aging: Toward a new understanding of the aging self. Ann Rev Gerontol Geriatr. 2015; 35: 1-28.

9. Westerhof GJ, Wurm S. Longitudinal research on subjective aging, health, and longevity: Current evidence and new directions for research. Ann Rev Gerontol Geriatr. 2015; 35: 145165.

10. Barak B, Stern B. Subjective age correlates: A research note. Gerontologist. 1986; 26: 571-578.

11. Havighurst RJ, Albrecht R. Older people. Oxford: Longmans, Green; 1953.

12. Knoll N, Rieckmann N, Scholz U, Schwarzer R. Predictors of subjective age before and after cataract surgery: Conscientiousness makes a difference. Psychol Aging. 2004; 19: 676-688.

13. Sargent-Cox KA, Anstey KJ, Luszcz MA. The relationship between change in self-perceptions of aging and physical functioning in older adults. Psychol Aging. 2012; 27: 750-760.

14. Mock SE, Eibach RP. Aging attitudes moderate the effect of subjective age on psychological well-being: Evidence from a 10-year longitudinal study. Psychol Aging. 2011; 26: 979- 986.

15. Galambos NL, Turner PK, Tilton-Weaver LC. Chronological and subjective age in emerging adulthood: The crossover effect. J Adol Res. 2005; 20: 538-556.

16. Montepare JM, Lachman ME. "You're only as old as you feel": Self-perceptions of age, fears of aging, and life satisfaction from adolescence to old age. Psychol Aging. 1989; 4: 73-78.

17. Levy BR. Mind matters: Cognitive and physical effects of aging self-stereotypes. J Gerontol Ser B Psychol Sci Soc Sci. 2003; 58: 203-211.

18. Rubin DC, Berntsen D. People over forty feel $20 \%$ younger than their age: Subjective age across the lifespan. Psychonomic Bull Rev. 2006; 13: 776-780.

19. Kleinspehn-Ammerlahn A, Kotter-Grühn D, Smith J. Self-perceptions of aging: Do subjective age and satisfaction with aging change during old age?. J Gerontol Ser B Psychol Sci Soc Sci. 2008; 63: 377-385.

20. Uotinen V. Age identification: A comparison between Finnish and North-American cultures. Int J Aging Human Develop. 1998; 46: 109-124.

21. Westerhof GJ, Barrett AE. Age identity and subjective well-being: A comparison of the United States and Germany. J Gerontol Ser B Psychol Sci Soc Sci. 2005; 60: 129-136.

22. Swann WB, Bosson JK. Self and identity. Handbook Soc Psychol. 2010; 2: 589-628.

23. Gergen KJ, Gergen MM. The new aging: Self-construction and social values. New York: Springer; 2000. p. 281-306.

24. Sneed JR, Whitbourne SK. Identity processing and self-consciousness in middle and later adulthood. J Gerontol Ser B Psychol Sci Soc Sci. 2003; 58: 313-319.

25. Heckhausen J, Schulz R. Motivation and self-regulation across the life span. Cambridge: Cambridge University Press; 1998. p. 50-77.

26. Fry CL. Handbook of aging and the social sciences. 4th ed. San Diego: Academic Press; 1996. P. 117-136.

27. Uotinen, V. Age identification: A comparison between Finnish and North-American cultures. Int J Aging Human Develop. 1998; 46: 109-124.

28. Ota H, Harwood J, Williams A, Takai J. A cross-cultural analysis of age identity in Japan and the United States. J Multilingual Multicultural Develop. 2008; 21: 33-41. 
29. Westerhof GJ, Barrett $A E$, Steverink N. Forever young? A comparison of age identities in the United States and Germany. Res Aging. 2003; 25: 366-383.

30. Westerhof GJ, Whitbourne SK, Freeman GP. The aging self in a cultural context: The relation of conceptions of aging to identity processes and self-esteem in the United States and the Netherlands. J Gerontol Ser B Psychol Sci Soc Sci. 2011; 67: 52-60.

31. Westerhof GJ, Miche M, Brothers AF, Barrett AE, Diehl M, Montepare JM, et al. Meanings of ageing and old age. London: Sage Publication; 2007.

32. Keyes CL, Westerhof GJ. Chronological and subjective age differences in flourishing mental health and major depressive episode. Aging Mental Health. 2012; 16: 67-74.

33. Weiss $D$, Lang FR. Thinking about my generation: Adaptive effects of a dual age identity in later adulthood. Psychol Aging. 2009; 24: 729-734.

34. Weiss D, Lang FR. "They" are old but "I" feel younger: Age-group dissociation as a selfprotective strategy in old age. Psychol Aging. 2012; 27: 153-163.

35. Keyes CL, Shmotkin D, Ryff CD. Optimizing well-being: The empirical encounter of two traditions. J Personality Soc Psychol. 2002; 82: 1007-1022.

36. Ryff CD, Singer BH. The exploration of happiness. Springer: Dordrecht; 2008. p. 97-116.

37. Ryff CD. Psychological well-being revisited: Advances in the science and practice of eudaimonia. Psychother Psychosom. 2014; 83: 10-28.

38. Heidrich SM, Ryff CD. The role of social comparisons processes in the psychological adaptation of elderly adults. J Gerontol. 1993; 48: 127-136.

39. Spuling SM, Miche M, Wurm S, Wahl HW. Exploring the causal interplay of subjective age and health dimensions in the second half of life. Eur J Health Psychol. 2013; 21: 5-15.

40. Stephan Y, Chalabaev A, Kotter-Grühn D, Jaconelli A. "Feeling younger, being stronger": An experimental study of subjective age and physical functioning among older adults. J Gerontol Ser B Psychol Sci Soc Sci. 2012; 68: 1-7.

41. 2008 physical activity guidelines for Americans: Be active, healthy, and happy! US Department of Health and Human Services; 2008. Available from: http://www.health.gov/paguidelines

42. Levy BR, Slade MD, Kasl SV. Longitudinal benefit of positive self-perceptions of aging on functional health. J Gerontol Ser B Psychol Sci Soc Sci. 2002; 57: 409-417.

43. Midlife in the United States: A National study of Health and Well-being. Subjective aging. (n.d.) Available from: http://www.midus.wisc.edu/newsletter/A2\%20Subjective\%20Aging\%20Brochure.pdf

44. Löckenhoff CE, De Fruyt F, Terracciano A, McCrae RR, De Bolle M, Costa PT, et al. Perceptions of aging across 26 cultures and their culture-level associates. Psychol Aging. 2009; 24: 941954.

45. Hughes DL, Shweder RA. Midlife in the United States (MIDUS): Survey of Minority Groups. Ann Arbor: Inter-university Consortium for Political and Social Research; 1995-1996. Available from: https://doi.org/10.3886/ICPSR02856.v4

46. Barrett $A E$. Socioeconomic status and age identity: The role of dimensions of health in the subjective construction of age. J Gerontol Ser B Psychol Sci Soc Sci. 2003; 58: 101-109.

47. Bookwala J, Boyar J. Gender, excessive body weight, and psychological well-being in adulthood. Psychol Women Quart. 2008; 32: 188-195. 
48. Greenfield EA, Reyes L. Continuity and change in relationships with neighbours: Implications for psychological well-being in middle and later life. J Gerontol Ser B Psychol Sci Soc Sci. 2014; 70: 607-618.

49. Son J, Wilson J. Volunteer Work and Hedonic, Eudemonic, and Social Well-Being. Sociol Forum. 2012; 27: 658-681.

50. Ware J, Sherbourne C. The MOS 36-item short-form health survey (SF-36): I. conceptual framework and item selection. Med Care. 1992; 30: 473-483.

51. Preacher KJ, Hayes AF. SPSS and SAS procedures for estimating indirect effects in simple mediation models. Behav Res Meth Instrum Comput. 2004; 36: 717-731.

52. Öberg P, Tornstam L. Youthfulness and fitness-identity ideals for all ages?. J Aging Identity. 2001; 6: 15-29.

53. Cooper $\mathrm{P}$, Thomas $\mathrm{E}$, Stevens $\mathrm{S}$. Subjective time experience in an intergenerational sample. Int J Aging Human Develop. 1981; 13: 183-193.

54. Morales E. Lesbians and gays in couples and families: A handbook for therapists. San Francisco: Jossey-Bass; 1996. p. 272-297.

55. O'Neil JM. Male sex role conflicts, sexism, and masculinity: Psychological implications for men, women, and the counseling psychologist. Couns Psychol. 1981; 9: 61-80.

56. Villereal GL, Cavazos Jr A. Shifting identity: Process and change in identity of aging MexicanAmerican males. J Sociol Soc Welfare. 2005; 32: 33-42.

57. Arciniega GM, Anderson TC, Tovar-Blank ZG, Tracey TJ. Toward a fuller conception of machismo: Development of a traditional machismo and Caballerismo scale. J Couns Psychol. 2008; 55: 19-33.

58. Lachman ME, Agrigoroaei S. Promotiong functional health in midlife and old age: Long-term protective effects of control beliefs, social support, and physical exercise. PLoS ONE. 2010; 5: e13297.

59. Rogler LH, Cortes DE, Malgady RG. Acculturation and mental health status among Hispanics: Convergence and new directions for research. Am Psychol. 1991; 46: 585-597.

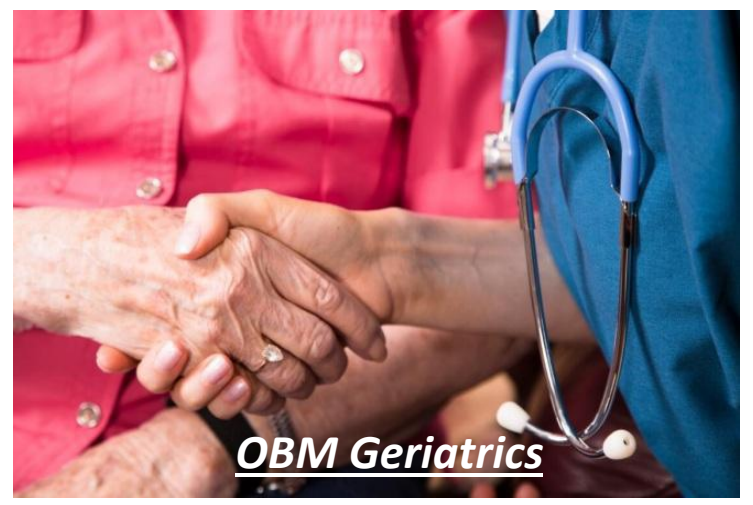

Enjoy OBM Geriatrics by:

1. Submitting a manuscript

2. Joining in volunteer reviewer bank

3. Joining Editorial Board

4. Guest editing a special issue

For more details, please visit: http://www.lidsen.com/journals/geriatrics 


\section{Editorial}

\section{Depression and Aging: Role of Social Support}

Ray Marks ${ }^{1,2, *}$

1. Department of Health and Human Performance, School of Health Sciences and Professional Studies, City University of New York, York College, NY 11451, United States;

2. Department of Health and Behavior Studies, Teachers College, Columbia University, NY 10027, United States; E-Mail: rm226@tc.columbia.edu

* Correspondence: Ray Marks; E-Mail: rm226@tc.columbia.edu

Special Issue: Depression and Aging: Role of Social Support

OBM Geriatrics

2019, volume 3, issue 1

doi:10.21926/obm.geriatr.1901042
Received: March 25, 2019

Accepted: March 26, 2019

Published: March 28, 2019

\section{Keywords}

Depression; aging; social support

Depression is a serious mood disorder associated with persistent feelings of sadness, loss of interest and pleasure in daily activities [1]. In addition to feelings of hopelessness, and low selfworth, individuals suffering from depression may experience poor sleep patterns, fatigue, excessive catastrophizing, interference with their daily activities, and appetite loss [1].

These symptoms, which may occur independently, or in reaction to the persistent presence of other illnesses, and/or adverse life events and losses, including mobility losses, can all influence the extent of suffering and disability as well as life quality associated with aging. Fortunately, depression can be reasonably well-diagnosed by taking a careful history, and by applying one or more validated scales to examine if indeed the individual is depressed, and if so, how severe the condition is.

However, even though research shows severe forms of depression affect 2-5\% of the United States population, and up to $20 \%$ may suffer from milder forms of the illness, especially after 70 years of age [2], or if they suffer from medical problems, and/or chronic disabling pain, measures

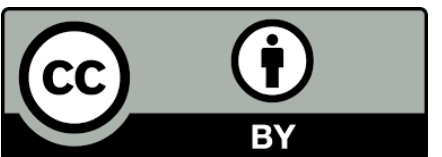

(C) 2019 by the author. This is an open access article distributed under the conditions of the Creative Commons by Attribution License, which permits unrestricted use, distribution, and reproduction in any medium or format, provided the original work is correctly cited. 
are not commonly put in place to identify depression, nor to treat the prevailing depression expected to prevail in older people in a holistic or integrated way. In particular, those suffering from severe depression [3], as well as those with more minor depression are not always evaluated for the important role of social support in moderating or mediating or offsetting the presence of depression.

Indeed, while the onset of depression may be genetic in origin, vulnerability towards depression may be increased by stress, loneliness, and isolation, among other factors, such as pain and chronic disability [4,5]. Since the treatments available for depression are not always efficacious, even if the condition is recognized, and unrelieved depression can be extremely injurious to the individual, efforts to prevent or ameliorate this condition among older adults are not only highly sought, but are of increasing importance in fostering successful aging. Alternately, in addition to a lower than desirable life quality, a failure to adequately attenuate depression among the elderly is strongly associated with a greater the risk of incurring a premature death, higher rates of inflammation, appetite changes, increased blood pressure, memory challenges, excess pain, a decreased desire for physical activities, weakness, trouble sleeping, anxiety, social withdrawal, and higher rates of osteoporosis, among other factors [2], including the overuse of existing health services.

Since depression is one of the most important predictors of health practitioner visits $[5,6]$, and can lead to suicide, as well as considerable physical, and social disability, minimizing, preventing or treating depression is strongly indicated among the older population. As well, given the fact that many elderly people live in poverty, and social adversity complicates the management and treatment of depression, through its impact on social network factors, interventions that can address depression as well as related social problems that exacerbate this are strongly indicated [6].

Social support, which is a term referring in part to the availability of help from others, if help is desired, as well as feelings or beliefs one is cared for, loved, esteemed and valued, a strong health determinant [7] is found to impact depression negatively and adversely, if this is unavailable or of poor quality. Key forms of social support, which are emotional, appraisal, informational and instrumental support [7], if mobilized appropriately are independently and collectively linked to being a stress buffer, can arguably influence overall wellbeing, and health outcomes positively and significantly. Alternately, the lack of adequate social support is linked to higher levels of depression than not, and may reflect a lack of psychological and material resources, as well as direct assistance and feedback by others, limitations in access to needed social services, counselling, psychotherapy, medications, cognitive behavioural therapy, and exercise opportunities.

Conversely, research points to the positive value of the application of one or more forms of social support in the context of either direct assistance, indirect assistance, tangible and intangible aspects of support in efforts to impact physical as well as mental health, as well as health behaviors positively and significantly.

Unsurprisingly, Nam et al. [8] identified social support as an important variable not only for older adults with chronic illness who lived alone, but for those with symptoms of depression, who may also suffer from suicidal ideation, a finding supported by Koizumi et al. [9]. Olagunju al. [10] argued that considering the burden of depression in the elderly and the influential roles of social support especially from family and significant others on depression; strengthening of informal 
social support and formal social support for the elders is advocated. In addition, design of community based geriatric mental health with social services and articulation of public policy to address old age needs are implied both by this group and Nagoor et al. [11].

Geriatric depression is a mental and emotional disorder affecting many older adults, that results in trouble concentrating, hopelessness, feelings of worthlessness, reduced motivation, decreased drive and reward for pleasurable activities, too much or too little sleep, doom and suicidal thoughts. Depression in older adults can hence reduce quality and years of life, especially if social support and the provision or development of a supportive environment or atmosphere is unavailable or perceived to be negative. Conversely, although late life depression is associated with a significant burden of disease [6], including the perception of inadequate received social support [12], the careful design of formal and informal social supports may prove helpful in alleviating the depression burden [10].

In the belief that concerted efforts to examine the role of social support in the context of geriatric depression, functional disability [13-15], this special edition focuses on the role of social support in explaining the burden of depression and its relationship and its amelioration, a relatively underreported topic.

Articles of any genre concerning the importance of social support in preventing and treating geriatric depression and its correlates are specifically sought.

\section{Author Contributions}

The author did all the works.

\section{Competing Interests}

The author has declared that no competing interests exist.

\section{References}

1. Edwards RR, Cahalan C, Mensing G, Smith M, Haythornthwaite JA. Pain, catastrophizing, and depression in the rheumatic diseases. Nat Rev Rheumatol. 2011; 7: 216-224.

2. Alexopoulos GS. Depression in the elderly. Lancet. 2005; 365: 1961-1970.

3. López-López A, Montorio I, Izal M, Velasco L. The role of psychological variables in explaining depression in older people with chronic pain. Aging Ment Health. 2008; 12: 735-745.

4. Alpass FM, Neville S. Loneliness, health and depression in older males. Aging Ment Health. 2003; 7: 212-216.

5. Areán PA, Mackin S, Vargas-Dwyer E, Raue P, Sirey JA, Kanellopolos $D$, et al. Treating depression in disabled, low-income elderly: A conceptual model and recommendations for care. Int J Geriatr Psychr. 2010; 25: 765-769.

6. Gonçalves-Pereira $M$, Prina AM, Cardoso AM, da Silva JA, Prince $M$, Xavier $M$, et al. The prevalence of late-life depression in a Portuguese community sample: A 10/66 Dementia Research Group study. J Affect Disord. 2019; 246: 674-681.

7. Cobb S. Social support as a moderator of lifestress. Psychosom Med. 1976; 38: 300-314. 
8. Nam EJ, Lee JE. Mediating effects of social support on depression and suicidal ideation in older korean adults with hypertension who live alone. J Nurs Res. 2018. doi: 10.1097/jnr.0000000000000292.

9. Koizumi Y, Awata S, Kuriyama S, Ohmori K, Hozawa A, Seki T, et al. Association between social support and depression status in the elderly: Results of a 1-year community-based prospective cohort study in Japan. Psychiatry Clin Neurosci. 2005; 59: 563-569.

10. Olagunju AT, Olutoki MO, Ogunnubi OP, Adeyemi JD. Late-life depression: Burden, severity and relationship with social support dimensions in a West African community. Arch Gerontol Geriatr. 2015; 61: 240-246.

11. Nagoor K, Darivemula SB, Reddy NB, Patan SK, Deepthi CS, Chittooru CS. Prevalence of mental illness and their association with sociodemographic factors in the rural geriatric population in Chittoor, Andhra Pradesh, India: A community-based study. J Educ Health Promot. 2018; 7: 165.

12. Rajapakshe OBW, Sivayogan S, Kulatunga PM. Prevalence and correlates of depression among older urban community-dwelling adults in Sri Lanka. Psychogeriatrics. 2018. doi: 10.1111/psyg.12389.

13. Travis LA, Lyness JM, Shields CG, King DA, Cox C. Social support, depression, and functional disability in older adult primary-care patients. Am J Geriatr Psychiatry. 2004; 12: 265-271.

14. Patra P, Alikari V, Fradelos EC, Sachlas A, Kourakos M, Rojas Gil AP, et al. Assessment of depression in elderly. Is perceived social support related? a nursing home study: depression and social support in elderly. Adv Exp Med Biol. 2017; 987: 139-150.

15. Steffens DC, Hays JC, Krishnan KR. Disability in geriatric depression. Am J Geriatr Psychiatry. 1999; 7: 34-40.

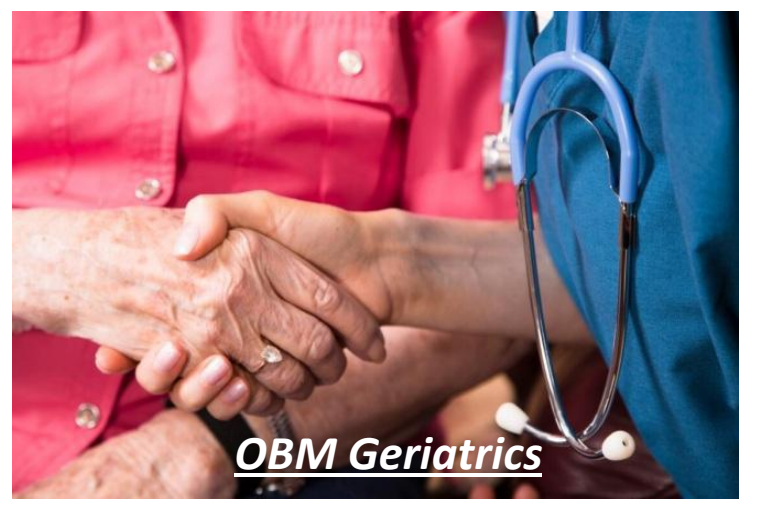

Enjoy OBM Geriatrics by:

1. Submitting a manuscript

2. Joining in volunteer reviewer bank

3. Joining Editorial Board

4. Guest editing a special issue

For more details, please visit: http://www.lidsen.com/journals/geriatrics 
Review

\title{
Association of Telomere Length, a Cellular Aging Marker, with Depression, PTSD and Hostility
}

Lei Zhang ${ }^{1,}{ }^{*}$, Xian-Zhang $\mathrm{Hu}{ }^{1}$, Xiaoxia $\mathrm{Li}^{1}$, Jacob Dohl ${ }^{2}$, Tianzheng $\mathrm{Yu}^{2}$, Robert J. Ursano ${ }^{1}$

1. Center for the Study of Traumatic Stress, Department of Psychiatry, Uniformed Services University of the Health Sciences, Bethesda, MD 20814, USA; E-Mails: lei.zhang@usuhs.edu; xianzhang.hu.ctr@usuhs.edu; xiaoxia.li@usuhs.edu; robert.ursano@usuhs.edu

2. Consortium for Health and Military Performance, Department of Military and Emergency Medicine, Uniformed Services University of the Health Sciences, Bethesda, MD 20814, USA; EMails: jacob.dohl.ctr@usuhs.edu; tianzheng.vu.ctr@usuhs.edu

* Correspondence: Lei Zhang; E-Mail: lei.zhang@usuhs.edu

Academic Editor: Michael Fossel

Special Issue: Perspectives on Telomeres and Aging

OBM Geriatrics

2019, volume 3 , issue 1

doi:10.21926/obm.geriatr.1901041
Received: December 28, 2018

Accepted: March 12, 2019

Published: March 21, 2019

\begin{abstract}
Depression, PTSD, and hostility are common mental conditions that are associated with aging. A growing body of research has highlighted the possible effects of depression, PTSD, and hostility on aging and telomere length ( $T L)$, a cellular aging marker. Individuals who exhibit excessive responses to stressors show notable circulating inflammatory responses with high cortisol reactivity, which increases cell turnover and oxidative stress and may subsequently contribute to shortened TL. This review focuses on the most recent discoveries in the relationship between TL and depression, PTSD and/or hostility, particularly in a unique military population (US active service members during the Afghanistan and Iraq wars). Current findings indicate that there is a solid association between leucocyte TL (LTL) and psychological conditions, such as depression, PTSD, and hostility. Future work should include controlled studies to test these associations.
\end{abstract}

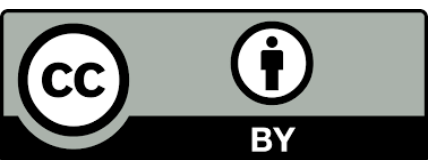

(C) 2019 by the author. This is an open access article distributed under the conditions of the Creative Commons by Attribution License, which permits unrestricted use, distribution, and reproduction in any medium or format, provided the original work is correctly cited. 


\section{Keywords}

Telomere length; cellular aging marker; depression; PTSD; hostility

\section{Introduction}

Mental disorders may be associated with aging. Accumulated evidence show that aging is a biological process in which there is a progressive telomere functional decline within the tissue [1, 2]. However, there are limited information available on aging biomarkers in mental disorders. Recently, several studies demonstrate a solid association between telomere length (TL) and mental conditions including depression, post-traumatic stress disorder, and hostility. In human, telomeres are tandem TTAGGG repeats at the ends of chromosomes, which are incompletely replicated during cellular division, eventually resulting in decreased telomere function. The decline in telomere function can result in an inability for cells to sustain replication and division and has consequences for aging $[1,2]$. The relationships between $T L$ and senescence have been demonstrated in cultured human cells. Hermann Muller was the first to name the "telomere" in the 1930s, describing it as a region of nonsense nucleotide sequences found at the end of each chromosome [3]. Without these special end structures, chromosomes would fuse and often break upon mitosis, resulting in chromosome instability which is detrimental to cells [3]. Muller and McClintock demonstrated that the basic roles of "telomeres" is providing chromosome stability, and ensuring faithful segregation of genetic material into daughter cells upon cell division [3]. Telomeres protect chromosomes from degradation, fusion, and recombination [4].The average TL is set and maintained in cells of the germline. In somatic cells, TL declines with age, contributing not only to overall cell loss, but incrementally progressive cellular dysfunction. With each cell division, TL declines, which can ultimately impact cell survival unless counteracted or reversed by the telomere-lengthening enzyme, telomerase [4-6]. Telomerase works by combining the function of an active enzyme, telomerase reverse transcriptase (TERT or hTERT in humans), with an essential RNA sequence, telomerase RNA component (hTERC), which provides the template for hTERT to generate additional tandem DNA repeats, in addition to several other stabilization and localization proteins including dyskerin, NHP2, NOP10 and GAR1. While certain cells including germ, stem, and cancer cells, demonstrate telomerase activity through the use of telomere maintenance mechanisms, somatic cells generally show little to no telomerase activity and are thus susceptible to TL shortening. Shortening of TL increases cellular susceptibility to apoptosis and cell death [7]. It is generally accepted that when telomeres are too short, DNA replication machinery cannot copy chromosome ends completely. As telomeres become critically short, both protein turnover and DNA repair proteins also begin to be down-regulated, resulting in an increase in DNA damage and denatured proteins (as well as the secondary results, such as mitochondrial inefficiency, lipid membrane defects, etc.) [8]. Subsequently, an "end replication problem" occurs and further cell division is impaired. Thus, telomeres have been repeatedly shown to determine cell senescence, i.e., the Hayflick limit, as resetting telomere length resets cell senescence [9-15].

$\mathrm{TL}$ is not only a robust indicator of human biological age (as distinguished from chronological age), but also a potential marker of disease risk showing in the earliest clinically oriented works [16-20]. TL may represent a cumulative log of factors such as the number of cell divisions and of 
exposure to cytotoxic processes such as excessive oxidation and inflammation [21-27]. In brief, the shortening of telomeres is associated with a number of diseases, from atherosclerosis to dementia $[16,17]$.

Recently, knowledge of the role telomeres play in diseases has advanced. In addition to the role of telomeres in cell fate and aging by adjusting the cellular response to biological stress, telomeres also play a role in response to psychological stress, which has been tested using leukocytes [28, 29]. In fact, TL shows both tissue and cell specificity. For example, shorter telomeres in leukocytes have been linked to psychosocial stress, depression, and PTSD [28]. Leukocytes TL (LTL) is significantly negatively correlated with lifetime depression. Chronically stressed individuals (e.g., maternal caregivers of chronically ill children and family caregivers of demented individuals) showed significantly shorter LTL compared to age matched controls [28, 29]. LTL is negatively correlated with the duration of caregiving. Women with a greater cumulative duration of caregiving stress had shorter telomeres [30]. Accelerated LTL shortening in individuals with Major Depressive Disorder (MDD) and PTSD could help explain the increased of medical morbidity seen in depression [7, 16, 21, 25, 31, 32] and accelerated aging in PTSD [33]. In addition, decreased in LTL is associated with an increase in hostility $[1,2]$.

The majority of studies on TL in mental disorders including MDD and Alzheimer's disease (AD) are based on leukocyte DNA. Subjects with AD had significantly shortening of LTL [34]. For example, shortened LTL is associated with risks for dementia and mortality [35] [36], and shorter LTL is a risk for earlier onset of dementia in females. Shorter LTL is also causally associated with a higher risk for AD [37]. There is a good correlation between TL in glial cell (particularly microglial cell) and CNS disease, such as AD [38]. There are potential implications of glial cell shortening for other CNS syndromes. Moreover, it is possible TL shortening is related to dementia [39]. Human subjects with high brain amyloid loads show a significantly higher degree of microglial dystrophy than amyloid-free controls [40]. In addition, the expression of serum telomere repeat binding factor 1 (TRF1) is significantly higher in patients with $A D$ than that of the controls [41]. The duration of $A D$ is significantly negatively correlated with telomere length [40].

In a rat model, significant telomere shortening and reduction of telomerase activity are also observed in microglia during normal aging. Telomere shortening correlates with microglial cell senescence. Mood stabilizer lithium effects the maintenance of TL in mouse model. Chronic lithium treatment was associated with longer telomeres in the samples from hippocampus and in the parietal cortex of triple-transgenic mouse model (3xTg-AD) compared to wild type mouse [42]. Here, we focus on the discussion of findings of the relationships between LTL and depression, PTSD, and hostility.

\section{Telomeres and Depression}

Major Depressive Disorder (MDD) is the most common psychiatric disorder and one of the leading causes of disability around the world. The worldwide number of subjects living with depression is estimated to be 322 million, $4.4 \%$ of the world's population [43]. The burden of depression reaches far beyond both mental condition and quality of life, extending to somatic health. It has been noted to not only increase the risk of cardiovascular diseases, stroke, diabetes and obesity morbidity, but also aging-related medical problems [43]. The association of depression and aging may partly be explained by processes of accelerated cellular aging, such as deterioration 
of telomere maintenance mechanisms [44]. TL is significantly negatively correlated with the duration of long-term depression and was inversely correlated with both inflammation and oxidative stress in depressed subjects [16, 32]. Average TL in depressed subjects who were above the median for long-term depression was 281 base pairs shorter than that in controls, corresponding to approximately seven years of accelerated cell aging [30]. Compared to healthy controls, subjects suffering from MDD had significantly shortened TL [16]. Shortening TL in MDD represents approximately 6-8 years of "accelerated aging" [45]. In addition, individuals with MDD at baseline have greater LTL shortening over two years than individuals without MDD, even after controlling for differences in age, sex, and body mass index [46]. Those with remitted MDD also had shorter TL than controls [47]. Moreover, MDD is associated with smaller right hippocampal volume. When corrected for age, sex, diagnostic group and total brain volume, TL was no longer associated with left or right hippocampal volume. These data indicate that cellular and neural processes may be mechanistically distinct during adolescence [48]. It is possible that TL shortening and hippocampal volume reduction are already present in early-onset MDD subjects [48]. Another study shows that MDD patients with diabetes who took Actos, an anti-diabetic drug specializing in lowering blood sugar levels, responded better to anti-depressant treatment and has longer telomeres, possibly indicating that lower blood sugar levels can result in less damage to both telomeres and the brain [49]. The shortened TL in depression may relate to the elevation of oxidative stress [50-53] and associates with a pro-inflammatory milieu [54-57], which is observed in PTSD and in preclinical models $[55,58-61]$. TL shortening is more likely to result in secondary epigenetic shifts, which can occur in the duration of depression or throughout a subject's lifetime $[30,62-67]$.

Those studies demonstrate novel insights into the relationship between depression and LTL. Shortened LTL is related to not only the MDD episode, but also the remitted stage. The mechanism for LTL shortening in MDD may involve the elevation of oxidative stress and pro-inflammatory milieu. In addition, Actos-induced LTL increase provides a possibility to identify drugs, which have new indication for alleviating aging induced LTL shortening. Further study is needed to elucidate whether increased TL shortening is the consequence of TL repair mechanisms failing to recover after a depressive episode, the result of long-term effects on cellular aging, or whether an underlying putative third factor, such as inflammation, altered metabolism, or shared genetic effects, impacts depression vulnerability, and accelerates TL shortening [68].

\section{Telomeres and PTSD}

TL is also associated with PTSD, which has been linked with biological aging as well [69, 70]. PTSD is an anxiety disorder that develops in individuals after exposure to traumatic stress and commonly accompanies age-related diseases, such as cardiovascular, autoimmune, neurodegenerative diseases, and early mortality [56, 71-74]. Repeated and prolonged stress responses potentially result in an accelerated rate of TL shortening. Higher levels of the stress hormone cortisol and catecholamines are associated with shorter TL $[75,76]$. Short LTL is also observed in both PTSD patients and subjects who experienced multiple categories of childhood trauma. A possible mechanism for TL shortening in PTSD is an inflammatory response with dysregulation of the hypothalamic-pituitary-adrenal (HPA) axis and sympathetic nervous system activation [77-80]. It is possible that inflammation may promote telomere shortening by increasing 
cell turnover and promoting the release of reactive oxygen species, which can damage telomeric DNA via oxidative stress $[69,81]$. The dysfunction of the HPA axis and inflammatory response may increase the risk of PTSD and the susceptibility to accelerated shortening of LTL. It seems early life traumatic experiences shape later life biological stress responses [82, 83]. It has been an accepted recommendation that cellular senescence may play roles in epigenetic and inflammation as well as induction of ROS. For example, the senescence possibly promotes inflammation [84] and increases both ROS and the ROS/ATP ratio as mitochondria lose efficiency due to decreased turnover of required aerobic enzymes and increasingly leaky mitochondrial lipid membranes $[85,86]$.

A recent study shows that, among the soldiers that served during the Iraq and/or Afghanistan war, participants with PTSD have shorter LTL than non-PTSD controls (Figure 1a) [33]. This data indicates that TL might be a potential blood based biomarker for PTSD. The younger non-PTSD controls who experienced more stressful life events tend to have longer LTL (Figure $1 \mathrm{~b}$ and $\mathrm{c}$ ). A negative association between LTL and age is observed in the non-PTSD controls, but not in PTSD subjects (Figure 1d). These data suggest that the mechanism of LTL was not the same in the PTSD and non- PTSD groups. It is possible that the age effects are not detectable in the PTSD group because they already have shorter telomeres, making it difficult to detect an additional age effect. However, LTL's are inherently unreliable as a measure of immune stress unless followed over long time intervals (e.g., months to years). The interpretation of these data should be further explored and/or determined.

There are several possible explanations for the relationships between LTL and PTSD. First, PTSD subjects with shorter LTL may be those with poor resilience for both PTSD and stress-related cellular aging. These data suggest that stress-related changes in telomere integrity may be one possible mechanism linking psychosocial stress and age-related disease [16, 25, 28, 67, 81, 87]. Second, those non-PTSD subjects who experienced more stressful life events and did not have a significant reduction of LTL may be a population resilient against both stress-related cellular aging and PTSD. That there are no significant differences in TL between subjects with and without childhood trauma events suggests that childhood trauma is not uniquely associated with changes in $\mathrm{TL}$ in that population. However, these findings contrast with a report [67] that childhood trauma was associated with changes in TL. These contradictory findings may be attributable to sampling differences (soldiers versus civilians), types of trauma exposure, or other factors including measuring current versus lifetime PTSD [67].

Taking all together, although substantial evidence indicates that TL alteration is associated with both traumatic stress and PTSD occurrence, further comprehensive research design is needed to test those contradictory findings. 


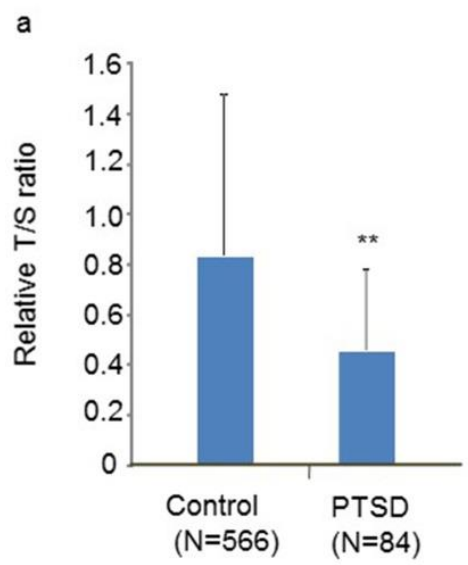

c

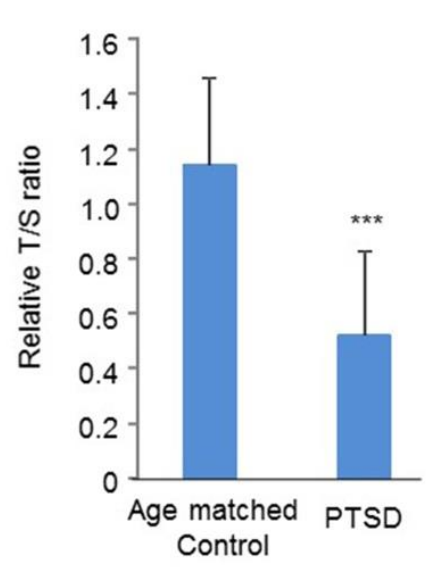

b

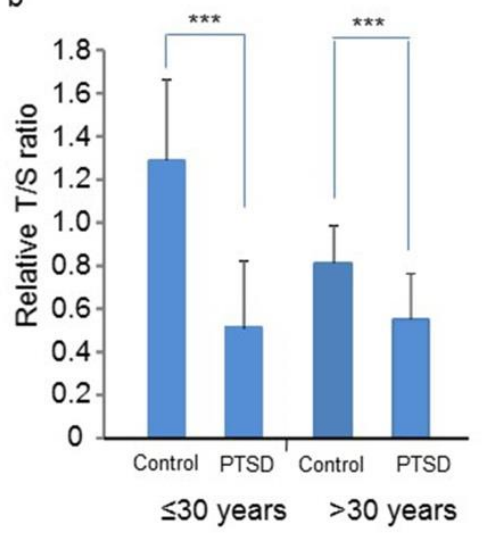

d

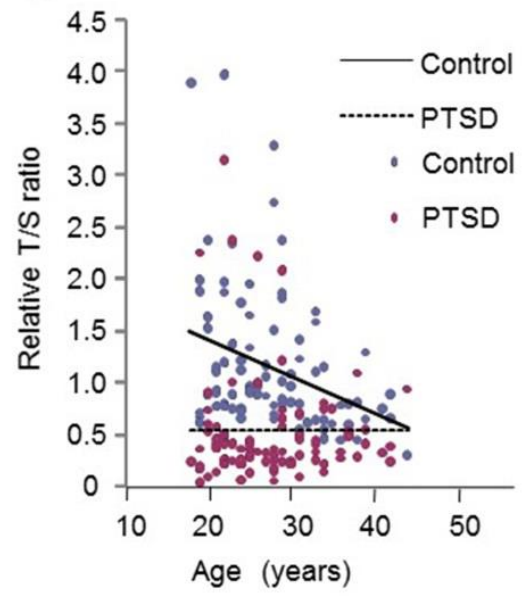

Figure 1 The telomere repeat copy number to single gene copy number (T/S) in subjects with and without PTSD. a. The relative T/S ratio in all subjects with or without PTSD. Subjects with PTSD $(n=84)$ had significantly lower relative $T / S$ ratio than non-PTSD controls $(n=566)$. b. The Relative T/S ratio in the PTSD and non-PTSD control aged $\leqslant 30$ or $>30$ years. c. Relative T/S ratio was significantly lower for the PTSD subjects $(n=84)$ compared with age-matched non-PTSD controls $(n=84)$. $d$. The relationships between age and relative T/S ratio in PTSD subjects and age-matched non-PTSD controls. The association of relative T/S ratio with age was observed in non-PTSD controls $(n=84 ; r=-$ $0.32, p<0.05)$. Such relationship was not obtained in the PTSD subjects $(n=84 ; r=-0.03$, $p>0.05$ ). The curves in different colors represent expected $T / S$ ratio for the proportion of non-PTSD subjects. Relative T/S ratio of subjects with PTSD was presented as open circles. $* * p<0.01 ; * * * p<0.001$.

\section{Telomeres and Hostility}

In addition to the association of TL with depression and PTSD, it is known that shortened telomeres are significantly associated with greater levels of hostility. Hostility is an enduring personality trait that reflects a predisposition to exhibit interpersonal animosity and mistrust. Hostility is associated with negative health outcomes, including age-related disorders [88, 89]. 
Individuals reporting higher-levels of hostility have shorter LTL than those reporting lower levels of hostility $[1,2]$.

A growing body of research has highlighted the possible effects of hostility and anger on cortisol and inflammatory marker levels $[90,91]$. Individuals who exhibit strong psychological reactions, including hostility, to environmental stressors show notable inflammatory responses and high cortisol reactivity, which increases cell turnover and oxidative stress and may subsequently contribute to shortened TL [64, 92-98]. Research further suggests that hostile individuals are more prone to undertake risky health behaviors (e.g., alcohol and tobacco), which also lessen TL [99-102]. There are varying levels of prevalence across sub-populations and demographics, and therefore warrant focused attention. For example, the prevalence rates of negative emotions (i.e., hostility and anger) and mental health outcomes (i.e., PTSD) increase in military populations following an operational deployment $[103,104]$. Such outcomes may serve as an underlying factor driving the acceleration of age-related health outcomes amongst military veterans $[1,96]$.

Recently, we have investigated the relationship between hostility, post-traumatic stress and LTL in a sample of United States Army Special Operations personnel $(n=474)$ who were deployed to Iraq and/or Afghanistan as part of combat operations. Hostility was measured with five items from the Brief Symptom Inventory (BSI). LTL was assessed using quantitative polymerase chain reaction methods, and linear regression analyses were conducted to determine the association of hostility and telomere length. We found that PTSD subjects reported higher hostility scores compared with those without PTSD. In addition, those reported high hostility score had shorter TL.

Univariate regression showed that the total score of hostility was negatively associated with LTL (95\% Cl: -0.06 to -0.002 , Beta $=-0.095, p<0.039$ ) and the presence of a significant correlation between $\mathrm{LTL}$, hostility impulse $(95 \% \mathrm{Cl}:-0.108$ to -0.009 , Beta= $-0.106, \mathrm{p}<0.021)$, and hostility control (95\% Cl: -0.071 to -0.002 , Beta $=-0.095, p<0.004)$. Multiple linear regression analyses revealed that hostility control does not enter the equation, while hostility impulse was still negatively correlated with the LTL $(p=0.021)$. Our data indicate that LTL is associated with hostility impulse.

In summary, the data particularly from a unique military sample demonstrate that TL shortening is associated with hostility, especially with impulsive hostility. Although the molecular mechanism for the interaction of TL with hostility is still unknown, prevention and treatment efforts designed to reduce hostility may help mitigate risk for LTL shortening, a process of cellular aging, and thus slow accelerated aged-related health outcomes.

\section{Conclusions}

This review presents direct evidence that short TL is associated with depression, PTSD, and hostility, particularly within PTSD subjects who have a substantial history of childhood trauma. One study demonstrates that even physically healthy young to middle-aged adults with PTSD and childhood trauma bear markers of cellular aging. However, another study within a military population did not find significant differences in TL between subjects with and without childhood trauma events. Those diverse results may be due to several factors, such as different stressors, methods, sample populations (soldiers vs. civilians), and the stages of the disorder in the studies. The intricate involvement of telomeres in both aging and mental disorders, such as depression, 
PTSD, and hostility, ensures that pathways involving telomeres and telomerase will remain the subject of intensive studies for many years to come.

\section{Author Contributions}

Lei Zhang, Xian-Zhang Hu, Xiaoxia Li, Jacob Dohl, Tianzheng Yu, and Robert J. Ursano wrote the manuscript. Lei Zhang and Xian-Zhang Hu contributed to the figures.

\section{Funding}

Sources of support in the form of grants: Center for the Study of Traumatic Stress, USUHS, USA.

\section{Competing Interests}

The authors have declared that no competing interests exist.

\section{References}

1. Watkins LE, Harpaz-Rotem I, Sippel LM, Krystal JH, Southwick SM, Pietrzak RH. Hostility and telomere shortening among u.S. Military veterans: Results from the national health and resilience in veterans study. Psychoneuroendocrinology. 2016; 74: 251-257.

2. Brydon L, Lin J, Butcher L, Hamer M, Erusalimsky JD, Blackburn EH, et al. Hostility and cellular aging in men from the whitehall ii cohort. Biol Psychiat. 2012; 71: 767-773.

3. Aubert G, Lansdorp PM. Telomeres and aging. Physiological reviews. 2008; 88: 557-579.

4. Blackburn EH. Structure and function of telomeres. Nature. 1991; 350: 569-573.

5. Blackburn EH. Telomere states and cell fates. Nature. 2000; 408: 53-56.

6. Beyne-Rauzy O, Prade-Houdellier N, Demur C, Recher C, Ayel J, Laurent G, et al. Tumor necrosis factor-alpha inhibits htert gene expression in human myeloid normal and leukemic cells. Blood. 2005; 106: 3200-3205.

7. Zhang $P$, Dilley $C$, Mattson MP. DNA damage responses in neural cells: Focus on the telomere. Neuroscience. 2007; 145: 1439-1448.

8. M. F. Cells, aging, and human disease. New York: Oxford University Press; 2004.

9. Harley $C B$, Futcher $A B$, Greider $C W$. Telomeres shorten during ageing of human fibroblasts. Nature. 1990; 345: 458-460.

10. Allsopp RC, Chang E, Kashefi-Aazam M, Rogaev El, Piatyszek MA, Shay JW, et al. Telomere shortening is associated with cell division in vitro and in vivo. Exp Cell Res. 1995; 220: 194-200.

11. Allsopp RC, Vaziri H, Patterson C, Goldstein S, Younglai EV, Futcher AB, et al. Telomere length predicts replicative capacity of human fibroblasts. P Natl Acad Sci USA. 1992; 89: 1011410118.

12. Hayflick L, Moorhead PS. The serial cultivation of human diploid cell strains. Exp Cell Res. 1961; 25: 585-621.

13. Bodnar AG, Ouellette M, Frolkis M, Holt SE, Chiu CP, Morin GB, et al. Extension of life-span by introduction of telomerase into normal human cells. Science. 1998; 279: 349-352.

14. Funk WD, Wang CK, Shelton DN, Harley CB, Pagon GD, Hoeffler WK. Telomerase expression restores dermal integrity to in vitro-aged fibroblasts in a reconstituted skin model. Exp Cell Res. 2000; 258: 270-278. 
15. Yang J, Nagavarapu U, Relloma K, Sjaastad MD, Moss WC, Passaniti A, et al. Telomerized human microvasculature is functional in vivo. Nat Biotechnol. 2001; 19: 219-224.

16. Simon NM, Smoller JW, McNamara KL, Maser RS, Zalta AK, Pollack MH, et al. Telomere shortening and mood disorders: Preliminary support for a chronic stress model of accelerated aging. Biol Psychiat. 2006; 60: 432-435.

17. Fitzpatrick AL, Kronmal RA, Gardner JP, Psaty BM, Jenny NS, Tracy RP, et al. Leukocyte telomere length and cardiovascular disease in the cardiovascular health study. Am J Epidemiol. 2007; 165: 14-21.

18. Fossel M. Telomerase and the aging cell: Implications for human health. JAMA: J Am Med Assoc. 1998; 279: 1732-1735.

19. Fossel M. Cell senescence in human aging: A review of the theory. In Vivo. 2000; 14: 29-34.

20. Banks DA, Fossel M. Telomeres, cancer, and aging. Altering the human life span. JAMA: J Am Med Assoc. 1997; 278: 1345-1348.

21. Blackburn EH, Greider CW, Szostak JW. Telomeres and telomerase: The path from maize, tetrahymena and yeast to human cancer and aging. Nat Med. 2006; 12: 1133-1138.

22. De Meyer T, Rietzschel ER, De Buyzere ML, Van Criekinge W, Bekaert S. Studying telomeres in a longitudinal population based study. Front Biosci. 2008; 13: 2960-2970.

23. Aviv A. Telomeres and human aging: Facts and fibs. Science of aging knowledge environment: SAGE KE. 2004; 2004: pe43.

24. Hezel AF, Bardeesy N, Maser RS. Telomere induced senescence: End game signaling. Curr Mol Med. 2005; 5: 145-152.

25. Epel ES. Psychological and metabolic stress: A recipe for accelerated cellular aging? Hormones (Athens). 2009; 8: 7-22.

26. Aviv A. Telomeres and human somatic fitness. J Gerontol A-Biol Sci Med Sci. 2006; 61: 871873.

27. Brouilette $S$, Singh RK, Thompson JR, Goodall AH, Samani NJ. White cell telomere length and risk of premature myocardial infarction. Arterioscl Throm Vasc Biol. 2003; 23: 842-846.

28. Epel ES, Blackburn EH, Lin J, Dhabhar FS, Adler NE, Morrow JD, et al. Accelerated telomere shortening in response to life stress. P Natl Acad Sci USA. 2004; 101: 17312-17315.

29. Damjanovic AK, Yang Y, Glaser R, Kiecolt-Glaser JK, Nguyen H, Laskowski B, et al. Accelerated telomere erosion is associated with a declining immune function of caregivers of alzheimer's disease patients. J Immunol. 2007; 179: 4249-4254.

30. Wolkowitz OM, Mellon SH, Epel ES, Lin J, Dhabhar FS, Su Y, et al. Leukocyte telomere length in major depression: Correlations with chronicity, inflammation and oxidative stress-preliminary findings. PloS one. 2011; 6: e17837.

31. Evans DL, Charney DS, Lewis L, Golden RN, Gorman JM, Krishnan KR, et al. Mood disorders in the medically ill: Scientific review and recommendations. Biol Psychiat. 2005; 58: 175-189.

32. Lung FW, Chen NC, Shu BC. Genetic pathway of major depressive disorder in shortening telomeric length. Psychiat Genet. 2007; 17: 195-199.

33. Zhang L, Hu XZ, Benedek DM, Fullerton CS, Forsten RD, Naifeh JA, et al. The interaction between stressful life events and leukocyte telomere length is associated with ptsd. Mol Psychiat. 2014; 19: 856-857.

34. Boccardi V, Arosio B, Cari L, Bastiani P, Scamosci M, Casati M, et al. Beta-carotene, telomerase activity and alzheimer's disease in old age subjects. Eur J Nutr. 2019. 
35. Herrmann M, Pusceddu I, Marz W, Herrmann W. Telomere biology and age-related diseases. Clin Chem Lab Med. 2018; 56: 1210-1222.

36. Scarabino D, Broggio E, Gambina G, Corbo RM. Leukocyte telomere length in mild cognitive impairment and alzheimer's disease patients. Exp Gerontol. 2017; 98: 143-147.

37. Zhan Y, Song C, Karlsson R, Tillander A, Reynolds CA, Pedersen NL, et al. Telomere length shortening and alzheimer disease--a mendelian randomization study. JAMA Neurol. 2015; 72: 1202-1203.

38. Flanary BE, Sammons NW, Nguyen C, Walker D, Streit WJ. Evidence that aging and amyloid promote microglial cell senescence. Rejuvenation Res. 2007; 10: 61-74.

39. Zhan Y, Hagg S. Telomere length shortening in alzheimer's disease: Procedures for a causal investigation using single nucleotide polymorphisms in a mendelian randomization study. Meth Mol Biol. 2018; 1750: 293-306.

40. Liu M, Huo YR, Wang J, Wang C, Liu S, Liu S, et al. Telomere shortening in alzheimer's disease patients. Ann Clin Lab Sci. 2016; 46: 260-265.

41. Wu Q, Han D, Zhang J, Li X. Expression of telomere repeat binding factor 1 and trf2 in alzheimer's disease and correlation with clinical parameters. Neurol Res. 2019: 1-6.

42. Cardillo GM, De-Paula VJR, Ikenaga EH, Costa LR, Catanozi S, Schaeffer EL, et al. Chronic lithium treatment increases telomere length in parietal cortex and hippocampus of tripletransgenic alzheimer's disease mice. J Alzheimers Dis. 2018; 63: 93-101.

43. Friedrich MJ. Depression is the leading cause of disability around the world. JAMA: J Am Med Assoc. 2017; 317: 1517.

44. Penninx BW, Milaneschi Y, Lamers F, Vogelzangs N. Understanding the somatic consequences of depression: Biological mechanisms and the role of depression symptom profile. BMC Med. 2013; 11: 129.

45. Hartmann N, Boehner M, Groenen F, Kalb R. Telomere length of patients with major depression is shortened but independent from therapy and severity of the disease. Depress Anxiety. 2010; 27: 1111-1116.

46. Vance MC, Bui E, Hoeppner SS, Kovachy B, Prescott J, Mischoulon D, et al. Prospective association between major depressive disorder and leukocyte telomere length over two years. Psychoneuroendocrinology. 2018; 90: 157-164.

47. Verhoeven JE, Revesz D, Epel ES, Lin J, Wolkowitz OM, Penninx BW. Major depressive disorder and accelerated cellular aging: Results from a large psychiatric cohort study. Mol Psychiat. 2014; 19: 895-901.

48. Henje Blom E, Han LK, Connolly CG, Ho TC, Lin J, LeWinn KZ, et al. Peripheral telomere length and hippocampal volume in adolescents with major depressive disorder. Transl Psychiat. 2015; 5: e676.

49. Rasgon N, Lin KW, Lin J, Epel E, Blackburn E. Telomere length as a predictor of response to pioglitazone in patients with unremitted depression: A preliminary study. Transl Psychiat. 2016; 6: e709.

50. Wolkowitz OM, Epel ES, Reus VI, Mellon SH. Depression gets old fast: Do stress and depression accelerate cell aging? Depress Anxiety. 2010; 27: 327-338.

51. Irie M, Miyata $M$, Kasai H. Depression and possible cancer risk due to oxidative DNA damage. J Psychiat Res. 2005; 39: 553-560. 
52. Forlenza MJ, Miller GE. Increased serum levels of 8-hydroxy-2'-deoxyguanosine in clinical depression. Psychosom Med. 2006; 68: 1-7.

53. Tsuboi H, Shimoi K, Kinae N, Oguni I, Hori R, Kobayashi F. Depressive symptoms are independently correlated with lipid peroxidation in a female population: Comparison with vitamins and carotenoids. J Psychosom Res. 2004; 56: 53-58.

54. Irwin MR, Miller AH. Depressive disorders and immunity: 20 years of progress and discovery. Brain Behav Immun. 2007; 21: 374-383.

55. Raison CL, Capuron L, Miller AH. Cytokines sing the blues: Inflammation and the pathogenesis of depression. Trends Immunol. 2006; 27: 24-31.

56. Cohen BE, Marmar CR, Neylan TC, Schiller NB, Ali S, Whooley MA. Posttraumatic stress disorder and health-related quality of life in patients with coronary heart disease: Findings from the heart and soul study. Arch Gen Psychiat. 2009; 66: 1214-1220.

57. Maes M, Yirmyia R, Noraberg J, Brene S, Hibbeln J, Perini G, et al. The inflammatory \& neurodegenerative (i\&nd) hypothesis of depression: Leads for future research and new drug developments in depression. Metab Brain Dis. 2009; 24: 27-53.

58. Leonard B. Stress, depression and the activation of the immune system. World J Biol Psychiat. 2000; 1: 17-25.

59. Aviv A. Telomeres, sex, reactive oxygen species, and human cardiovascular aging. J Mol Med (Berl). 2002; 80: 689-695.

60. Valdes AM, Andrew T, Gardner JP, Kimura M, Oelsner E, Cherkas LF, et al. Obesity, cigarette smoking, and telomere length in women. Lancet. 2005; 366: 662-664.

61. Ilmonen P, Kotrschal A, Penn DJ. Telomere attrition due to infection. PloS one. 2008; 3: e2143.

62. Prather AA, Puterman E, Lin J, O'Donovan A, Krauss J, Tomiyama AJ, et al. Shorter leukocyte telomere length in midlife women with poor sleep quality. J Aging Res. 2011; 2011: 721390.

63. Aviv A, Hunt SC, Lin J, Cao X, Kimura M, Blackburn E. Impartial comparative analysis of measurement of leukocyte telomere length/DNA content by southern blots and qpcr. Nucleic Acids Res. 2011; 39: e134.

64. O'Donovan A, Pantell MS, Puterman E, Dhabhar FS, Blackburn EH, Yaffe K, et al. Cumulative inflammatory load is associated with short leukocyte telomere length in the health, aging and body composition study. PloS one. 2011; 6: e19687.

65. Hoen PW, de Jonge P, Na BY, Farzaneh-Far R, Epel E, Lin J, et al. Depression and leukocyte telomere length in patients with coronary heart disease: Data from the heart and soul study. Psychosom Med. 2011; 73: 541-547.

66. Steptoe A, Hamer M, Butcher L, Lin J, Brydon L, Kivimaki M, et al. Educational attainment but not measures of current socioeconomic circumstances are associated with leukocyte telomere length in healthy older men and women. Brain Behave Immun. 2011; 25: 1292-1298.

67. O'Donovan A, Epel E, Lin J, Wolkowitz O, Cohen B, Maguen S, et al. Childhood trauma associated with short leukocyte telomere length in posttraumatic stress disorder. Biol Psychiat. 2011; 70: 465-471.

68. Verhoeven JE, Revesz D, Picard M, Epel EE, Wolkowitz OM, Matthews KA, et al. Depression, telomeres and mitochondrial DNA: Between- and within-person associations from a 10-year longitudinal study. Mol Psychiat. 2018; 23: 850-857. 
69. O'Donovan A, Hughes BM, Slavich GM, Lynch L, Cronin MT, O'Farrelly C, et al. Clinical anxiety, cortisol and interleukin-6: Evidence for specificity in emotion-biology relationships. Brain Behav Immun. 2010; 24: 1074-1077.

70. Heim C, Newport DJ, Mletzko T, Miller AH, Nemeroff CB. The link between childhood trauma and depression: Insights from hpa axis studies in humans. Psychoneuroendocrinology. 2008; 33: 693-710.

71. Durel LA, Carver CS, Spitzer SB, Llabre MM, Weintraub JK, Saab PG, et al. Associations of blood pressure with self-report measures of anger and hostility among black and white men and women. Health Psychol. 1989; 8: 557-575.

72. Boscarino JA, Forsberg CW, Goldberg J. A twin study of the association between ptsd symptoms and rheumatoid arthritis. Psychosom Med. 2010; 72: 481-486.

73. Yaffe K, Vittinghoff $E$, Lindquist $K$, Barnes D, Covinsky KE, Neylan T, et al. Posttraumatic stress disorder and risk of dementia among us veterans. Arch Gen Psychiat. 2010; 67: 608-613.

74. Qureshi SU, Pyne JM, Magruder KM, Schulz PE, Kunik ME. The link between post-traumatic stress disorder and physical comorbidities: A systematic review. Psychiat Q. 2009; 80: 87-97.

75. Parks CG, Miller DB, McCanlies EC, Cawthon RM, Andrew ME, DeRoo LA, et al. Telomere length, current perceived stress, and urinary stress hormones in women. Cancer Epidem Biomark Prev. 2009; 18: 551-560.

76. Epel ES, Lin J, Wilhelm FH, Wolkowitz OM, Cawthon R, Adler NE, et al. Cell aging in relation to stress arousal and cardiovascular disease risk factors. Psychoneuroendocrinology. 2006; 31: 277-287.

77. Hoge EA, Brandstetter K, Moshier S, Pollack MH, Wong KK, Simon NM. Broad spectrum of cytokine abnormalities in panic disorder and posttraumatic stress disorder. Depress Anxiety. 2009; 26: 447-455.

78. Young EA, Breslau N. Cortisol and catecholamines in posttraumatic stress disorder: An epidemiologic community study. Arch Gen Psychiat. 2004; 61: 394-401.

79. Yehuda R, Golier JA, Yang RK, Tischler L. Enhanced sensitivity to glucocorticoids in peripheral mononuclear leukocytes in posttraumatic stress disorder. Biol Psychiat. 2004; 55: 1110-1116.

80. Neylan TC, Brunet A, Pole N, Best SR, Metzler TJ, Yehuda R, et al. Ptsd symptoms predict waking salivary cortisol levels in police officers. Psychoneuroendocrinology. 2005; 30: 373381.

81. Miller GE, Rohleder N, Stetler C, Kirschbaum C. Clinical depression and regulation of the inflammatory response during acute stress. Psychosom Med. 2005; 67: 679-687.

82. Shonkoff JP, Boyce WT, McEwen BS. Neuroscience, molecular biology, and the childhood roots of health disparities: Building a new framework for health promotion and disease prevention. JAMA: J Am Med Assoc. 2009; 301: 2252-2259.

83. Hertzman C. The biological embedding of early experience and its effects on health in adulthood. Ann NY Acad Sci. 1999; 896: 85-95.

84. Lasry A, Ben-Neriah Y. Senescence-associated inflammatory responses: Aging and cancer perspectives. Trends Immunol. 2015; 36: 217-228.

85. Abdul-Ghani MA, Jani R, Chavez A, Molina-Carrion M, Tripathy D, DeFronzo RA. Mitochondrial reactive oxygen species generation in obese non-diabetic and type 2 diabetic participants. Diabetologia. 2009; 52: 574-582. 
86. Roussel $D$, Boel $M$, Mortz $M$, Romestaing $C$, Duchamp $C$, Voituron $Y$. Threshold effect in the h2o2 production of skeletal muscle mitochondria during fasting and refeeding. J Exp Biol. 2019; 222.

87. Liu Y, Bohr VA, Lansdorp P. Telomere, telomerase and aging. Mech Ageing Dev. 2008; 129: 12.

88. Elbogen EB, Wagner HR, Fuller SR, Calhoun PS, Kinneer PM, Mid-Atlantic Mental Illness Research E, et al. Correlates of anger and hostility in iraq and afghanistan war veterans. Am J Psychiat. 2010; 167: 1051-1058.

89. Miller TQ, Smith TW, Turner CW, Guijarro ML, Hallet AJ. A meta-analytic review of research on hostility and physical health. Psychol Bull. 1996; 119: 322-348.

90. Coccaro EF, Lee R, Coussons-Read M. Elevated plasma inflammatory markers in individuals with intermittent explosive disorder and correlation with aggression in humans. JAMA Psychiat. 2014; 71: 158-165.

91. Marsland AL, Prather AA, Petersen KL, Cohen S, Manuck SB. Antagonistic characteristics are positively associated with inflammatory markers independently of trait negative emotionality. Brain Behav Immun. 2008; 22: 753-761.

92. Graham JE, Robles TF, Kiecolt-Glaser JK, Malarkey WB, Bissell MG, Glaser R. Hostility and pain are related to inflammation in older adults. Brain Behav Immun. 2006; 20: 389-400.

93. Ranjit N, Diez-Roux AV, Sanchez B, Seeman T, Shea S, Shrager S, et al. Association of salivary cortisol circadian pattern with cynical hostility: Multi-ethnic study of atherosclerosis. Psychosom Med. 2009; 71: 748-755.

94. Smith TW, Glazer K, Ruiz JM, Gallo LC. Hostility, anger, aggressiveness, and coronary heart disease: An interpersonal perspective on personality, emotion, and health. J Personality. 2004; 72: 1217-1270.

95. Smith TW, Ruiz JM, Uchino BN. Mental activation of supportive ties, hostility, and cardiovascular reactivity to laboratory stress in young men and women. Health Psychol. 2004; 23: 476-485.

96. Brydon L, Strike PC, Bhattacharyya MR, Whitehead DL, McEwan J, Zachary I, et al. Hostility and physiological responses to laboratory stress in acute coronary syndrome patients. J Pychosom Res. 2010; 68: 109-116.

97. Chida $\mathrm{Y}$, Hamer M. Chronic psychosocial factors and acute physiological responses to laboratory-induced stress in healthy populations: A quantitative review of 30 years of investigations. Psychol Bull. 2008; 134: 829-885.

98. Suarez EC, Kuhn CM, Schanberg SM, Williams RB, Jr., Zimmermann EA. Neuroendocrine, cardiovascular, and emotional responses of hostile men: The role of interpersonal challenge. Psychosom Med. 1998; 60: 78-88.

99. Suzuki N, Nakanishi K, Yoneda M, Hirofuji T, Hanioka T. Relationship between salivary stress biomarker levels and cigarette smoking in healthy young adults: An exploratory analysis. Tob Induc Dis. 2016; 14: 20.

100. Appleton KM, Woodside JV, Arveiler D, Haas B, Amouyel P, Montaye M, et al. A role for behavior in the relationships between depression and hostility and cardiovascular disease incidence, mortality, and all-cause mortality: The prime study. Ann Behav Med. 2016; 50: 582-591. 
101. Kim D, Kubzansky LD, Baccarelli A, Sparrow D, Spiro A, 3rd, Tarantini L, et al. Psychological factors and DNA methylation of genes related to immune/inflammatory system markers: The va normative aging study. BMJ Open. 2016; 6: e009790.

102. Potijk MR, Janszky I, Reijneveld SA, Falkstedt D. Risk of coronary heart disease in men with poor emotional control: A prospective study. Psychosom Med. 2016; 78: 60-67.

103. McHugh T, Forbes D, Bates G, Hopwood M, Creamer M. Anger in ptsd: Is there a need for a concept of ptsd-related posttraumatic anger? Clin Psychol Rev. 2012; 32: 93-104.

104. Heesink L, Rademaker A, Vermetten E, Geuze E, Kleber R. Longitudinal measures of hostility in deployed military personnel. Psychiat Res. 2015; 229: 479-484.

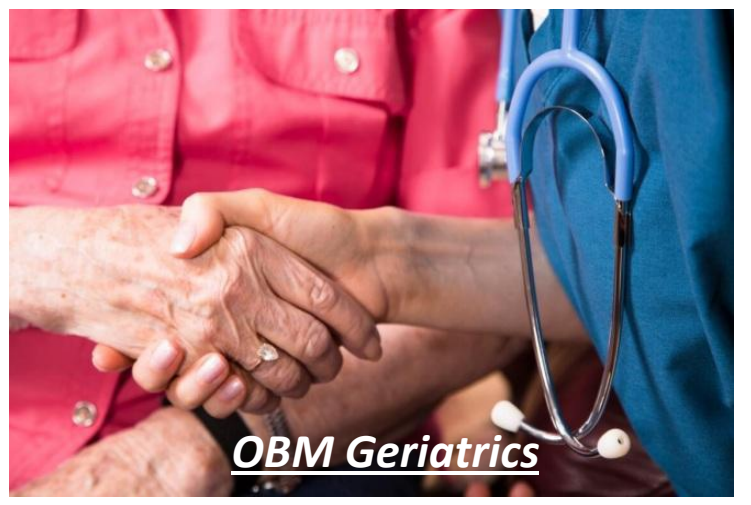

Enjoy $O B M$ Geriatrics by:

1. Submitting a manuscript

2. Joining in volunteer reviewer bank

3. Joining Editorial Board

4. Guest editing a special issue

For more details, please visit:

http://www.lidsen.com/journals/geriatrics 
Review

\title{
Does Dementia Increase the Risk of Dental Decay?
}

\author{
Robert J Emanuel $^{*}$
}

Haywards Heath Health Centre, Heath Road, Haywards Heath, West Sussex, RH16 3BB, UK; E-Mail: robert.emanuel@nhs.net

* Correspondence: Robert J Emanuel; E-Mail: robert.emanuel@nhs.net

Academic Editor: Michael Fossel

Special Issue: Treatment of Dementia

OBM Geriatrics

2019, volume 3, issue 1

doi:10.21926/obm.geriatr.1901040
Received: January 09, 2019

Accepted: March 20, 2019

Published: March 21, 2019

\begin{abstract}
The amount of patients who suffer with dementia is set to rise with an ageing population. As well as living longer, patients will be retaining their natural teeth into their later life and will be less reliant on dentures. From both the current evidence available in the literature, and anecdotally from clinicians experienced in treating patients with dementia, it can be concluded that on balance, patients with dementia appear to be at a greater risk from dental caries (dental decay). However, more research in this area would help produce a firmer evidence base to support this conclusion and should be encouraged. To improve clinical outcomes for patients with dementia, it is important for clinicians to ensure that patients have access to high quality preventive care which should include dietary advice and fluoride supplementation where appropriate to help mitigate this increased risk.
\end{abstract}

\section{Keywords}

Oral health; dementia; dental caries; dental caries risk; prevention

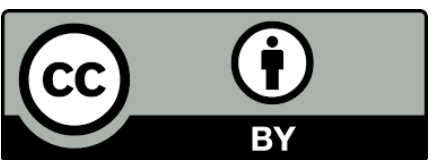

(C) 2019 by the author. This is an open access article distributed under the conditions of the Creative Commons by Attribution License, which permits unrestricted use, distribution, and reproduction in any medium or format, provided the original work is correctly cited. 


\section{Does Dementia Increase Dental Caries (Decay) Risk?}

The number of patients who suffer with dementia is set to rise with an ageing population [1]. As well as living longer, patients will be retaining their natural teeth longer and will be less reliant on dentures [2].

Although life expectancy is increasing, the amount of years a person may expect to live without chronic illness may not necessarily increase to the same extent. This phenomena is likely to have an effect of the provision of health care including dental, stretching the finite and limited resources set aside for dental care even further. Older patients will often suffer with worse oral health due to a complex mixture of additional risk factors [3]. It is envisaged that the effect of these risk factors will be exaggerated in patients with dementia who are no longer able to care for themselves as well as they may have in the past.

So will such a deterioration in general health also translate specifically into a higher risk of dental caries? Although risk from other oral diseases may also increase, it is dental caries where prevention is likely to be absolutely crucial to maintain good oral health. The common operative options of dental restoration for patients with advanced dementia may be impossible due to a subsequent decline in ability to cooperate with dental treatment with declining cognitive function.

\section{Does Dementia Increase Caries Risk?}

It would seem logical that a patient suffering with dementia, will also have an increased risk from developing dental caries. Reasons for this include, but not exclusively so, are:

- Impaired cognition, apathy and apraxia lead to a decline in the continued importance of good oral health behaviours [4].

- A change in dietary habit including an increase in sugar containing foods and drinks [5]?

- Poor cooperation leading to difficulties with maintaining oral hygiene [6].

- Difficulty with accessing dental care due to poor cooperation in certain cases.

The evidence from the literature presents a mixed picture when asking, "Does dementia increase caries risk?" For instance Philip et al 2012 [7] found that the number of decayed, missing and filled teeth (DMFT) for patients living in residential care with and without dementia were not significantly different, though the level of untreated caries between the groups was significant. Their conclusion was that the level of caries incidence was proportionate to the level of disability rather than diagnosis of dementia alone and were keen to stress the importance of prevention.

This trend for level of compromise being the main indicator of disease risk is also shown in a number of other studies. Srisilpanan and Jai-ua [8] found a strong correlation with severity of dementia and caries experience. In their cross sectional study of dementia patients attending a memory assessment clinic, they found that patients had better than average oral health when first accessing the clinic and when functionally independent. However, the severity of the dementia of these patients appeared to have a significant effect on caries experience which is suggestive of an increase in caries risk with cognitive decline.

Research teams led by Birita Ellefsen in Copenhagen also carried out research on patient groups attending memory assessment clinics. They found that patients with a low MMSE (Mini Mental State Examination ) score also had a higher incidence of caries [9]. When the patients were followed up, they were found to have an elevated risk of both dental caries in the crowns of teeth 
(coronal caries) and caries in the roots (root caries) one year after initial referral to the clinic [10]. The team also found that those with a diagnosis of non Alzheimers dementia had an even higher caries risk. Adam and Preston [11] examined the oral health of individuals with dementia in nursing homes in 2006, and could find no significant difference between the DMFT scores of those individuals with mild dementia and those with moderate or severe disease.

One observation in the literature is that much of the research lends itself to examination and recording of the oral health status of those with early and moderate dementia as those with severe dementia are more likely to be non-complaint and therefore their oral status is unable to be accurately recorded for research data [12]. This obviously has the potential to lead to bias in the results and may lead to under recording of the state of caries incidence experienced by a patient during their health journey with dementia.

\section{A Different Pattern of Disease?}

A literature review carried out by Chalmers and Pearson in 2005 [13] reported a different pattern of dental caries when compared to healthy older adults. Their findings demonstrated more coronal caries and retained carious roots as well as a higher prevalence of root caries. Ellefsen's team in Copenhagen concurred with this finding [14] stating that root caries was highly prevalent among patients with recently diagnosed dementia, suggesting a need for active assessment. Their research showed that $89 \%$ had at least one root caries or coronal lesions and $66 \%$ of the group had at least two. Root caries lesions can often be treated preventively rather than operatively with application of topical fluoride and improved oral hygiene. This makes early diagnosis paramount to the success of such treatment.

Chalmers et al in 2003 [15] looked at community dwelling patients, with or without dementia. As a baseline the patients with dementia had a significantly increased caries incidence when compared to those without the disease. As measured at the start, $22 \%$ of the dementia group had at least one carious surface coronally and $31 \%$ had at least one root caries lesion. These figures rose to $41 \%$ for coronal and $50 \%$ for root caries when reviewed at one year. Not only were these results significantly different from the non-dementia group but also hinted at a high rate of deterioration in the dementia group.

There are few longitudinal studies in the literature, but Ship and Puckett [16] in 1994 followed a small group of 21 patients with dementia and 21 without for a period of 2-3 years. The patients were all living in the community and were having no active medical treatment. Those with dementia were found to have decreased salivary flow rates and poorer oral hygiene. Additionally when compared to the non-dementia group, those with dementia had a (unsurprisingly) higher incidence of caries at the start of the study. However what was perhaps surprising was that there was no difference in the rate of deterioration between the groups at 2-3 years.

\section{Previous Systematic Reviews}

Wu et Al's systematic review in 2016 [17] examined 16 longitudinal studies, five of which addressed the question of do patients with dementia have a higher caries rate. They summarised that the number of cavities was higher in patients with dementia but wasn't significantly different due to small sample size. 
Foley et al produced a systematic review in 2017 [18] which concluded that of the 28 studies they examined, patients with dementia had more carious teeth and worse oral hygiene (including more bleeding gums, poor denture hygiene). However what also was apparent was that this was an area of dental research with a limited evidence base due to the small amount of studies carried out.

\section{Anecdotal Evidence}

The oral health status of patients with dementia is often discussed by dentists who regularly care for this group of patients. With the advent of people not only living longer but retaining their teeth as well, clinicians who deal with patients with dementia are often presented with patients with severely broken down dentitions including extensive root caries, retained roots and secondary caries around existing restorations.

The usual operative treatment options for managing caries such as fillings or crowns after removal of diseased tooth tissue, is in many cases just not a viable treatment option for a lot of patients who suffer with dementia. As well as possibly having limited cooperation they additionally often present with additional risk factors such as poor, uncontrolled diet; unrestorable remaining tooth tissue and difficulties with maintaining oral hygiene. Uncontrolled disease will lead to poor survival times for any repairs carried out.

It is therefore evident that for these patients, preventive dental care is of the utmost importance.

\section{Prevention Strategies for Improving Oral Health and Reducing Disease Incidence}

Delivering Better Oral Health (DBOH) [19] is a toolkit produced by Department of Health, Public Health England and The British Association for the Study of Community Dentistry. The latest edition is the third edition, published March 2017.

This is the current gold standard for providing an evidence base for UK dental practice and deals with advice for general patients and those with an increased risk of dental disease, including those patients with dementia.

With reference to reducing caries risk, it has sections on:

- Increasing fluoride availability. (Table 1)

- Healthy eating advice. (Table 1 )

Additionally the Seattle Care Pathway [20] offers more specific advice on managing prevention in older patients including those with dementia. This innovative pathway was developed after a three day conference in Seattle in 2013 , and takes into account the different levels of frailty experienced by the patient as they age, and the increased dependency they have on care being provided by others. It considers patients to have five levels of dependency ranging from none, to high, and provides different advice on different actions needed to maintain oral health including assessment, prevention, treatment and communication.

For prevention (Table 2) there is an emphasis, as with $\mathrm{DBOH}$ on increasing fluoride availability but also covers the use of antibacterial agents such as Chlorhexidine and care planning for oral hygiene. For those deemed high risk, oral hygiene strategies to reduce the risk of respiratory infections are also mentioned as there is now a growing consensus that poor periodontal health can be a contributory factor for aspiration pneumonia [21]. 
Table 1 From delivering better oral health [19].

\section{Increasing Fluoride availability (patient).}

- Brush at least twice daily, with a fluoridated toothpaste.

- Use fluoridated toothpaste with at least 1350ppm fluoride.

- Brush last thing at night and at least on one other occasion.

- Spit out after brushing and do not rinse, to maintain fluoride concentration.

Increasing Fluoride availability for patients with increased caries risk (professional).

- Apply fluoride varnish to teeth twice yearly (2.2\% NaF).

- Use a fluoride mouthrinse daily $(0.05 \% \mathrm{NaF})$ at a different time to brushing.

- For those with obvious active coronal or root caries prescribe 2,800 or 5000 ppm fluoride toothpaste.

\section{Healthy eating advice (patient).}

- The frequency and amount of sugary food and drinks should be reduced.

Oral Hygiene is also a crucial factor in maintaining oral health in the elderly population. An observation study of care home residents by Zander and Boniface in 2017 [22], however showed that of 161 residents observed, 93 (58\%) did not have their teeth or dentures brushed. Such a disturbing scenario will hopefully be reversed though once the new NICE (National Institute for Health and Care Excellence) guidelines which were introduced in 2016 for "Oral health for adults in care homes" [23] have had time to be assimilated as part of the culture of care provided by care homes. (see table 3.)

The guidelines give advice on:

- Care home policies on oral health.

- Oral Health assessment and care planning.

- Daily mouth care.

- Care staff knowledge and skills.

- Availability of local oral health service.

- Oral health promotion services.

- General dental practices and community dental services.

And it's hope their introduction will ensure that care providers for those who aren't able to necessarily look after their oral health, will be better informed and trained to deliver daily oral care. 
Table 2 Prevention" from Seattle Care Pathway [20].

\section{No dependency Pre-dependency}

Develop

homecare plan

to prevent or

control oral

infection, pain

and dysfunction. content.
Consider prescribing for caries toothpastes, varnishes, gels and/or mouthrinses with a high (>or=5000ppm of F) fluoride

\section{Peridodontitis, antibacterial}

toothpaste, professional cleaning, chlorhexidine products (not longterm use in this group due to side effects), Oral Hygiene Instruction.

Oral cancer: risk modification and education.

Tooth surface loss: risk modification, sensitivity products as indicated, tooth/mouthguard. Develop daily oral care plan.

Mucositis: advised denturewearers to leave dentures out of the mouth in a dry environment when sleeping.
Low dependency

Assess the cause for the impact on oral health. Base preventive strategies on mitigating the aggravating factors

Adjust methods of delivering pre-dependency prescriptions as needed (e.g. modified toothbrush handles or electric toothbrush).

Assess risk of adverse effects of polypharmacy (e.g. dry mouth; sugars in medication) Offer relief from dry mouth as required with water spray in an atomiser bottle, chewing gum, salivary substitutes or pilocarpine.

Monitor effectiveness of the daily care plan.
Medium dependency

Maintain contact with other members of the

interprofessional healthcare team to monitor and help their contributions to the oral health regimens.

\section{Reassess the need to increase}

the concentration of fluoride in toothpastes and mouthrinses.

Prescribe application of preventive and therapeutic products (e.g. fluoride varnishes, chlorhexidine rinses) by nurses and other staff.

Reassess risk of adverse effects from polypharmacy (e.g. dry mouth; sugars in medication).

Reassess need for relief from dry mouth and effectiveness of daily oral care plan.
High dependency

Focus on the increasing challenges of preventing and managing oral infections and disorders and of controlling pain and comorbidity, such as respiratory infections and dry mouth.

Emphasise management of pain and infection.

Maintain use of highly concentrated fluoride toothpastes, mouthrinses, varnishes and gels.

\section{Expand preventive} strategies to manage severe mucositis and the growing risk of respiratory infections. 
Table 3 Advice for care home staff taken from NICE guidance [23].

These recommendations are for managers of care staff who support daily personal care.

Ensure care staff provide residents with daily support to meet their mouth care needs and preferences, as set out in their personal care plan after their assessment. This should be aligned with the advice in the Delivering Better Oral Health toolkit, including:

- brushing natural teeth at least twice a day with fluoride toothpaste

- providing daily oral care for full or partial dentures (such as brushing, removing food debris and removing dentures overnight)

- using their choice of cleaning products for dentures if possible

- using their choice of toothbrush, either manual or electric/battery powered

- daily use of mouth care products prescribed by dental clinicians (for example, this may include a high fluoride toothpaste or a prescribed mouth rinse, see NICE's guideline on managing medicines in care homes)

- daily use of any over-the-counter products preferred by residents if possible, such as particular mouth rinses or toothpastes; if the resident uses sugar-free gum, consider gum containing xylitol.

Ensure care staff know which member of staff they can ask for advice about getting prescribed mouth care products, or helping someone to use them.

Ensure care staff know how to recognise and respond to changes in a resident's mouth care needs.

Ensure care staff know how to respond if a resident does not want daily mouth care or to have their dentures removed (see NICE's website page on your care). 


\section{Follow Up Clinical Provision and Evaluation}

Although a lot of evidence based advice exists on how best to support good oral health in patients with dementia, there still appears to be a lack of this advice being followed in day to day health care practice [24].

In the general health care setting it is important to raise awareness that many older patients now do in fact retain their teeth for longer and these teeth are potentially more vulnerable to dental decay as well as other oral diseases. Additionally these doctors, nurses and therapists need to know where to direct their patients for dental advice. Historically the majority of patients receiving care in their later years would have by that stage in their life lost all their teeth and probably worn dentures which arguably need less input and maintenance. It is also important for all health care providers to understand the benefit of a healthy diet and where possible this diet should have a low frequency of sugar to ensure oral health is also optimised. The author plans to develop a resource which can be used to provide information and guidance to health care providers and patients to help fill in any current gaps in their knowledge.

Additionally dental health professionals need to hold greater importance for preventive dental care in this group of patients. The problem with state funded healthcare schemes is that good preventive practice is not always sufficiently funded leaving the dentist insufficient time to ensure that good prevention is embedded in a patient's dental treatment plan.

One unique solution may be to develop a bespoke service where patients with dementia can be referred to receive specific advice on good dental prevention by a service run by dental professionals (dentists, hygienists and dental nurses). This bespoke service would provide a useful resource for general health care workers to send patients whom they may not feel confident advising on dental issues; as well as a useful service to support general dental practice where time may not be available to provide the desperately needed prevention advice. Such a service would need to be carefully evaluated to prove its worth in terms of outcomes for patients and cost effectiveness.

\section{Summary}

From both the current evidence available in the literature, and anecdotally from clinicians experienced in treating patients with dementia, it can be concluded that on balance, patients with dementia appear to be at a greater risk from dental caries. However, more research in this area would help produce a firmer evidence base to support this conclusion and should be encouraged.

To improve clinical outcomes for patients with dementia, it is important for clinicians to ensure that patients have access to high quality preventive care which should include dietary advice and fluoride supplementation where appropriate to help mitigate this increased risk.

\section{Author Contributions}

The author did all the works.

\section{Competing Interests}

The author has declared that no competing interests exist. 


\section{References}

1. Ghezzi EM, Ship JA. Dementia and Oral Health. Oral Surg Oral Med Oral Path Radiol Endod. 2000; 8: 2-5.

2. NHS Digital. Adult Dental Health Full report 2009. (Cited 2017 November 20) http://content.digital.nhs.uk/pubs/dentalsurveyfullreport09

3. What is Known About the Oral Health of Older People in England and Wales A review of oral health surveys of older people. Public Health England/ The British Association for the Study of Community Dentistry 2015. (Cited $2017 \quad$ November 20) https://www.gov.uk/government/uploads/system/uploads/attachment_data/file/489756/W hat_is_known_about_the_oral_health_of_older_people.pdf

4. Friedlander AH, Norman DC, Mahler ME, Norman KM, Yagiela JA. Alzheimer's disease Psychopathology, medical management and dental implications. JADA. 2006; 137: 1240-1251.

5. Morris $\mathrm{CH}$, Hope RA, Fairburn CG. Eating habits in dementia - a descriptive study. Brit J Psychiat. 1989; 154: 801-806.

6. Delwel S, Binnekade TT, Perez RSGM, Hertogh CMPM, Scherder EJA, Lobbezoo F. Oral hygiene and oral health in older people with dementia: A comprehensive review with focus on oral soft tissues. Clin Oral Invest. 2018; 22: 93-108.

7. Philip $P$, Rogers $C$, Kruger $E$, Tennant $M$. Caries experience of institutionalised elderly and it's association with dementia and functional status. Int J Dent Hyg. 2012: 10: 122-127

8. Srisilapanan P, Jai-Ua C. Oral health status of dementia patients in Chiang Mai Neurological Hospital. J Med Assoc Thai. 2013; 96: 351-357.

9. Ellefsen B, Holm-Pedersen P, Morse DE, Schroll M, Andersen BB, Waldemar G. Caries prevalence in older persons with and without dementia. J Am Ger Soc. 2008; 56: 59-67.

10. Ellefsen B, Holm-Pedersen P, Morse DE, Schroll M, Andersen BB, Waldemar G. Assessing caries increments in elderly patients with and without dementia $A$ one-year follow-up study. JADA. 2009; 140: 1392-1400.

11. Adam H, Preston AJ. The oral health of individuals with dementia in nursing homes. Gerodontology. 2006; 23: 99-105.

12. Fiske J, Frenkell H, Griffiths J, Jones V. Guidelines for the development of local standards of oral health care for people with dementia. Gerodonotology. 2006; 23: 3-32.

13. Chalmers J, Pearson A. Oral hygiene care for residents with dementia: A literature review. J Adv Nurs. 2005; 52: 410-419.

14. Ellefsen BS, Morse DE, Waldemar G, Holm-Pedersen P. Indicators for root caries in Danish persons with recently diagnosed Alheimer's disease. Gerodontology. 2012; 29: 194-202.

15. Chalmers JM, Carter KD, Spencer AJ. Oral diseases and conditions in community-living older adults with and without dementia. Spec Care Dentist. 2003; 23: 7-17.

16. Ship JA, Puckett SA. Longitudinal study on oral health in subjects with Alzheimer's disease. J Am Ger Soc. 1994; 42: 57-63.

17. Wu B, Fillenbaum GG, Plassman BL, Guo L. Association between oral health and cognitive status: A systematic review. J Am Geriatr Soc. 2016; 64: 739-751.

18. Foley NC, Affoo RH, Siqueria WL, Martin RE. A systematic review examining the oral health status of persons with dementia. JDR Clin Transl Res. 2017; 2: 330-342. 
19. https://www.gov.uk/government/publications/delivering-better-oral-health-an-evidencebased-toolkit-for-prevention

20. Pretty IA, Ellwood RP, Lo ECM, MacEntee MI, Muller F, Rooney E, et al. The Seattle Care Pathway for securing oral health in older patients. Gerodontology. 2014; 31: 77-87.

21. Awano S, Ansai T, Takata Y, Soh I, Akifusa S, Hamasaki T, et al. Oral health and mortality risk from pneumonia in the elderly. J Dent Res. 2008; 87: 333-339.

22. Zander A, Boniface D. Directly observed daily mouth care provided to care home residents in one area of Kent, UK. Community Dent Heal. 2017; 34: 32-36.

23. Oral health for adults in care homes. NICE 2016. https://www.nice.org.uk/guidance/ng48

24. Emanuel R, Sorensen A. A study of oral health prevention behaviours for patients with early stage dementia. Brit Dent J. 2018; 224: 38-42.

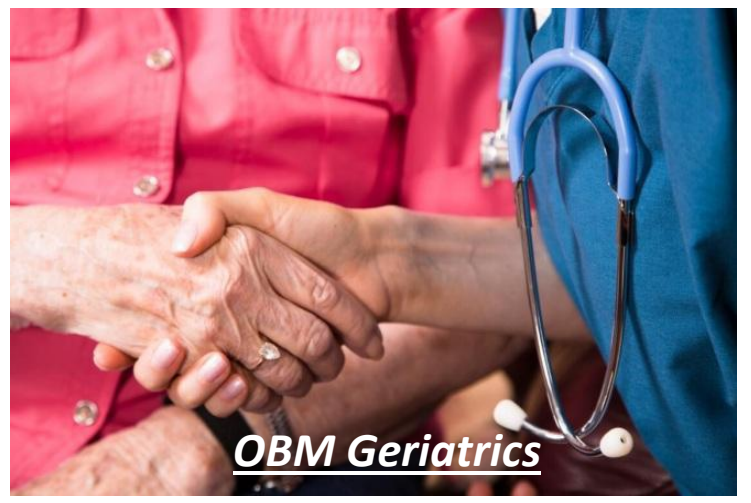

Enjoy OBM Geriatrics by:

1. Submitting a manuscript

2. Joining in volunteer reviewer bank

3. Joining Editorial Board

4. Guest editing a special issue

For more details, please visit:

http://www.lidsen.com/journals/geriatrics 
Perspective

\title{
Behavioural and Complementary Interventions for Healthy Neurocognitive Aging
}

\author{
Robert L. Conder, Jr. ${ }^{1,{ }^{+},{ }^{*}}$, Christopher Friesen ${ }^{2,+}$, Alanna A. Conder ${ }^{1,+}$
}

1. Carolina Neuropsychological Service, Raleigh, NC, USA; E-Mails: bconder10@gmail.com; aconder14@gmail.com

2. Niagara Neuropsychology, Grimsby, Ontario, Canada; E-Mail: dr.cfriesen@gmail.com

+ These authors contributed equally to this work.

* Correspondence: Robert L. Conder, Jr.; E-Mail: bconder10@gmail.com

Academic Editor: Kamen Tsvetanov

Special Issue: $\underline{\text { Health Modifiers of Neurocognitive Aging }}$

OBM Geriatrics

2019, volume 3 , issue 1

doi:10.21926/obm.geriatr.1901039
Received: November 30, 2018

Accepted: March 18, 2019

Published: March 20, 2019

\begin{abstract}
Background: While the percent of persons 65 and older is expected to increase to $17 \%$ of the world's population by 2050, this increase in longevity does not necessarily suggest a parallel increase in health status. While aging is an inevitable aspect of living, there are factors which can accelerate morbidity and mortality, as well as factors likely to promote a healthy transition into longevity. This paper addresses behavioural and complementary interventions for healthy neurocognitive aging. Specific evidence-based cardiovascular exercise guidelines, sleep hygiene strategies to improve restorative sleep, and dietary modifications to improve brain and heart health are reviewed. Also reviewed are neurofeedback and brain brightening neuromodulation interventions to limit mental declines often associated with aging. Research evidence tying behavioural and complimentary treatment interventions to improved outcomes in older persons with Alzheimer's and other dementias is also presented.
\end{abstract}

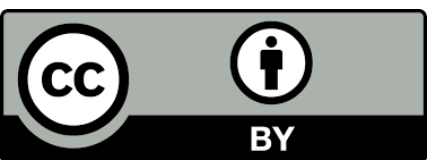

(C) 2019 by the author. This is an open access article distributed under the conditions of the Creative Commons by Attribution License, which permits unrestricted use, distribution, and reproduction in any medium or format, provided the original work is correctly cited. 
Methods: Evidence based perspective paper offered on the basis of clinical neuropsychological experience and review of relevant research literature.

Results: Evidence-based practice guidelines and research studies addressing behavioral and complementary interventions to support healthy neurocognitive aging were reviewed. Mounting research evidence reviewed herein supports identification of Exercise, Sleep and Diet/Nutrition as core health modifiers for healthy neurocognitive aging. Easy to adopt behavioural interventions as well as some innovative treatment interventions were reviewed to delay or minimize the normal physical and mental decline often associated with aging. Adjunct activities and interventions such as neurofeedback, neuromodulation, sauna and supplements being investigated also show some promise for healthy neurocognitive aging.

Conclusions: Normal aging does not inevitably portend acquisition of chronic health problems and faltering mental acuity. As we have attempted to show, avoidance of certain unhealthy habits and behaviors and adoption of healthy habit and behavioural alternatives can foster good mental and neurocognitive health throughout the lifespan.

\section{Keywords}

Aging; neurocognition; health; exercise; sleep; diet; neurofeedback; neuromodulation

\section{Introduction}

Worldwide, the population age 65 and older is growing exponentially [1]. The percent of persons 65 and older is 8.5 percent and is expected to increase to $17 \%$ of the world's population by 2050 , with the oldest adults living in North American and Europe. However, this increase in longevity does not necessarily suggest a parallel increase in health status. Additionally, for the current middle age population, life expectancy is shorter than that of current older adults. How can this be in a time of advances in medical care, diagnosis and treatment, and pharmaceuticals?

While aging is an inevitable aspect of living, there are factors which can accelerate morbidity and mortality, and factors likely to promote a healthy transition into longevity. Rates of childhood obesity are skyrocketing [2]. The cause is multifactorial. School systems are reducing emphasis on physical fitness and health due to financial cost and ostensibly a need for more classroom time to help students pass standardized tests [3]. Food companies spend billions of dollars marketing unhealthy foods to children, especially with Saturday morning cartoons and children's programming [4]. The rise in social media and computerized devices has increased sedentary behaviour and perhaps altered both healthy physical and cognitive development in children and adolescents [5]. Unfortunately, many of these sedentary and overweight children become prediabetic and pre-metabolic young adults, who have a higher likelihood of developing "heart disease, Type 2 diabetes, metabolic syndrome and many types of cancer" in middle-age [6]. There is mounting data that middle age sedentary behaviour, obesity, diabetes, and hypertension are precursors of earlier onset dementia [7].

As rehabilitation neuropsychologists who evaluate older adults for cognitive and memory disorders, it is dismaying to see the growing incidence of metabolic and cardiovascular disease associated with obesity and sedentary behaviours. Many of the dementias may be due to poor 
lifestyle choices, and not primary medical illnesses or genetics [8]. These lifestyle choices are modifiable. This paper will explore factors and interventions which have shown promise in promoting healthy neurocognitive aging and slowing abnormal neurocognitive aging such as dementia and age-related memory loss [9]. Primary areas covered will include what has been referred to as the "Holy Trinity" of positive behavioural interventions to promote healthy aging: Exercise, Sleep, and Diet. Ancillary interventions are also reviewed.

\section{Exercise}

Multiple organizations have developed guidelines for activity levels to reach therapeutic benefits from exercise. Numerous studies have documented the increase in neurocognitive performance from exercise interventions. The aetiology is not clear, but such factors as increases in brain derived neurotrophic factor, glial cell neurotrophic factor, lowering of insulin resistance, increases in cardiovascular and cerebrovascular blood flow, and increases in all neurotransmitter production and balance are among hypotheses studied. Cognitively, exercise is felt to increase working memory and executive functioning. Normalized neuroelectrical functioning of the brain (suggestive of better cognitive functioning) has been found with both intense and incidental everyday exercise. Psychologically, exercise has been associated with reductions in depression and anxiety as well as concomitant increases in self-esteem and quality of life. These psychological factors can lead to a greater sense of optimism and efficacy, prompting participation in other health promotion behaviours.

\subsection{Exercise Guidelines}

In their Physical Activity Guidelines for Americans $2^{\text {nd }}$ Edition [10], recently revised guidelines from the U.S. Department of Health and Human Services Center for Disease Control and Prevention retained the long-standing recommended requirements for cardiovascular exercise to receive therapeutic benefits for physical, psychological and neurocognitive health [11]. Globally, these recommendations were issued by the World Health Organization [12]. For adults under age 65 who do not have contraindicated medical illnesses, 150 minutes of moderate cardiovascular exercise per week is recommended. "Moderate" exercise is defined by the American Heart Association [13] as exercising at 50 to $70 \%$ of one's maximum heart rate for age. Alternatively, the Harvard T.H. Chan School of Public Health [14] grades exercise by exertion as measured in Metabolic Equivalent of Tasks or METS, with "Moderate" exercise in the 3 to 6 METS range. The recommended exercise time can be reduced to 75 minutes per week if one exercises at a "Vigorous "level", defined as an exercise heart rate greater than $75-85 \%$ of one's age-adjusted maximum, or exercise exertion greater than 6 METS. Muscle strengthening activities involving the major muscle groups are recommended two or more days per week. For Older Adults age 65 and greater, the time recommendations double to 300 minutes of moderate cardiovascular exercise per week, or 150 minutes of vigorous cardiovascular exercise, or some combination of the two levels of intensity. A new PAG-2 recommendation for the older group is for balance training, which is needed to counteract problems with gait and balance dysfunction with typical aging. Balance training also can help prevent or reduce fall risk associated with serious health consequences such as hip or vertebral fractures or geriatric traumatic brain injury. Further, more sophisticated forms of balance training such as tai chi are felt to improve executive functioning by exercising the 
prefrontal lobe cortex motor fields. One major change to the CDC PAG-2 recommendations is that the prior recommendation for at least a 10 consecutive minute period of moderate to vigorous physical activity (termed "bouted MVPA") has been dropped, as newer evidence shows positive effects for reduced metabolic syndrome with bouted and nonbouted but intense physical activity [15]. Because removal of the minimum 10 minute sustained duration guideline "encourages Americans to move more frequently throughout the day" [16], it has been well received as "a potential game-changer for boosting population-level physical activity...[as] people are now given permission to move in ways they prefer and that fit into their complex lives" [17].

\subsection{Benefits of Exercise}

In addition to reducing the incidence of chronic illness which can impair healthy aging, positive changes from exercising to a therapeutic level include increase in brain volume, brain-derived neurotrophic factor (BDNF), insulin sensitivity, cardiac health, heart rate variability, and healthy sleep and declines in blood pressure, LDL lipids, cancer risk, and risk of premature death [18-20] Psychological benefits of cardiovascular exercise to recommended levels include reduction in depression and anxiety and enhancement of self-esteem, self-efficacy, and quality of life [21-24]. Neurocognitive benefits have been identified for working memory, attentional processes and executive functioning [23-27]. Most promising, studies indicate that therapeutic exercise may decrease risk, onset time and/or effects of dementia $[9,28,29]$ and may normalize brain EEG against age-related declines in resting alpha peak frequency [30].

\subsection{Exercise Options}

The type of cardiovascular exercise depends on one's initial fitness level and any comorbid medical illnesses. Weight-bearing exercises such as walking, jogging, running, or exercising on a treadmill or elliptical are highly recommended [10]. In persons with ambulatory, balance or joint problems, options include nonweight-bearing exercise such as a rowing machine, Tai chi or lower impact exercises such as water aerobics [28]. If medically tolerated, the newer approach of High Intensity Interval Training (HIIT) optimizes time of work out, and may have benefits greater than steady-state exercise [31, 32]. It is postulated that HIIT affects memory abilities mediated by the hippocampus and executive abilities mediated by the frontal cortex. However, research is only currently being conducted to investigate the role of HIIT on Brain Derived Neurotrophic Factor (BDNF) and other mechanisms to support this hypothesis [20]. Incidental physical activity in older adults, such as yard work, housework and caretaking for a spouse or family member has been found to increase cognitive performance and normalize resting state EEG when performed at a sufficiently high level of intensity. High levels of incidental physical activity are positively associated with cognition and EEG activity in aging [33].

\section{Sleep}

\subsection{Sleep and Aging}

Normal aging adversely affects several parameters of sleep. Changes in sleep efficiency, total sleep time, and reductions in percentage of both Rapid Eye Movement (REM) sleep and slow wave sleep occurs [34]. These changes are usually associated with decrements in cognitive performance, 
such as attention, working memory, declarative memory, accuracy of performance, and reaction time [35]. Our modern lifestyles are in many ways incongruent with our evolutionary heritage for sleep. While body regulation may function optimally on a 24-hour circadian rhythm where we rise and sleep in line with the sun, technological advances and environmental changes have significantly disrupted our sleep-wake cycles and impinged on restful and restorative sleep. Many electrical devices (lights, TV's, computers, Smart Phones) give off significant amounts of blue wavelength light in the electromagnetic spectrum. When the brain perceives light, either natural or man-made, the suprachiasmatic nucleus will not signal the pineal gland to release melatonin, needed to begin the sleep cycle. This effect is likely worse in older adults given their already reduced ability to produce and release melatonin. Fortunately, there are many behavioural interventions which can address sleep dysfunction in older adults beyond the exercise guidelines outlined above.

\subsection{Benefits of Sleep Hygiene}

Normal aging adversely effects sleep onset and ability to reach deeper levels of restorative sleep. The primary behavioural intervention for primary insomnia is Cognitive Behavioural Therapy for Insomnia (CBT-I). CBT-I postulates that poor sleep involves cognitive distortions about sleep quality as well as poor sleep hygiene. Establishing and maintaining Sleep Hygiene includes implementation of simple behaviour changes such as: developing consistent times to go to bed and wake up, even on weekends; avoidance of daytime napping of greater than 15-20 minutes; restricted use of the bed for sleep and sex; and leaving the bedroom if sleep onset has not occurred within 20 minutes. Supportive approaches include reduction in late evening caffeine intake, alcohol consumption and stimulating exercise activities. The bedroom sleep environment can be modified to be more comfortable by using "black-out" shades or blinds; keeping the bedroom dark and cooled to about $65^{\circ} \mathrm{F}$; wearing blue light blocking glasses several hours before bed; and minimizing electronics that emit blue light. Finally, sleep onset and maintenance can be enhanced by practicing relaxation approaches such as mindfulness meditation, listening to relaxation tapes, and self-hypnosis.

Of importance to the aging population is emerging research suggesting a strong role of deep restorative sleep and the prevention of Alzheimer's Disease. A primary mechanism implicated is the newly-discovered glymphatic system in the brain that essentially washes the brain during deeper stages of sleep [36]. It is thought that this process removes amyloid-beta plaque byproducts characteristically seen in Alzheimer's. Implementation of sleep hygiene protocols may help offset reductions in deep slow wave delta sleep typically seen with aging, hopefully supporting this glymphatic clearance pathway.

\section{Diet and Nutrition}

While modern technology has adversely affected sleep quality, the modern diet has adversely affected basic health parameters, leading to increases in obesity and chronic illness. Such illnesses in the US and worldwide include diabetes, heart disease, stroke and some types of cancer [37]. As discussed, these illnesses can lead to abnormal brain aging, neurocognitive dysfunction, and poorer mental health. Among dietary approaches, adherence to the simple metabolic equation is 
a basic way to reduce the incidence of obesity: maintain an energy balance in which the number of calories consumed equals the number of calories used by the body for activities of daily living.

\subsection{Benefits of Dietary Modification}

While almost any dietary approach can be better than the modern commercial "fast food" diet, many commercial dietary systems focus on weight loss and not necessarily maintenance of a heart or brain healthy diet. A version of the Mediterranean diet, the "MIND" diet (Mediterranean Dietary Approaches to stop hypertension) has been researched rigorously and found to reduce the incidence of abnormal brain neuropathology [38]. The MIND Diet has also been found to reduce the incidence of Dementia, and conversion of Mild Cognitive Impairment to Dementia [39, 40]. The risk for Alzheimer's disease was reduced when the MIND Diet was paired with exercise. Longevity was increased by an average of four years or longer in persons who developed Alzheimer's while maintaining a healthy diet. On the other hand, a recent brain MRI study of older persons who survived mainly on a fast food commercial diet showed accelerated brain shrinkage, especially in the memory important hippocampus [41].

\subsection{Dietary Guidelines and Caveats for Older Adults}

As each person's dietary needs are individual due to a number of biological, social and medical factors, consultation with a registered dietitian or nutritionist is recommended to develop the best dietary plan. With this caveat in mind, research evidence generally associates positive health outcomes and reduced risk of heart disease, stroke and cancer with diets primarily made up of unprocessed foods such as fruits, vegetables, complex carbohydrates and healthy poly- and monounsaturated fats from sources such as nuts, seeds, olive oil, and avocados. While the Ketogenic diet (a high fat, low carbohydrate, modest protein diet) has shown efficacy in epilepsy and migraine, it has not been sufficiently researched in dementia or brain trauma and intuitively, it may not be beneficial. Similarly, Intermittent Fasting is a promising area of dietary research that involves time-restricted eating or fasting over a period of hours within a day, or days within a week. Two popular approaches are the 16/8 and 5:2 methods. In mice, where most of the research has been conducted, restricted eating improved neural connections in the hippocampus, which is instrumental in memory. Intermittent fasting may thus be a promising intervention for middle age persons adopting patterns to improve current health and minimize future risk of agerelated neurocognitive decline. However, it should be noted that the Alzheimer's Association does not recommend fasting for older persons, who may already be eating a diet altered by age-related changes in senses of smell and taste, and may become physically vulnerable due to improper nutrition. Similar concerns were noted for intermittent fasting for older persons with dementia and Parkinson's disease [39].

\section{Neurofeedback and Other Brain Training}

One biological measure of the functional activity of the human brain is the electroencephalogram (EEG). In normal aging, the EEG reflects changes which are felt to parallel decrements in intellectual abilities and cognitive processing in older adults. There is some controversy about whether these measured processes are normal variants of aging, or actually 
reflect a neuropathological process. Nevertheless, common findings on the EEG in older adults are characterized by "slowing of the alpha rhythm, increase in slow waves, and focal disturbance" [42]. These electrical measures are felt to reflect slowing of cognitive processing, decrements in working memory and executive functioning, and inter-hemispheric and intra-hemispheric communication difficulties. Neurofeedback and Brain Training approaches discussed below are utilized to modify EEG patterns correlated with adverse neurocognitive effects of aging.

\subsection{Background on Neurofeedback}

Traditionally, the EEG is a tool of clinical neurology which uses the International 10-20 topographic system to noninvasively measure the amplitude of four broad frequency bands over specific cortical brain sites, primarily to view significant pathology such as epilepsy, tumours, brain injury or metabolic disturbance. The EEG proceeded modern neuroimaging techniques such as $\mathrm{CT}$, MRI and PET scans by decades. Since the 1960s, biological processes in the body have been monitored, including heart rate, respiration, muscle tension, skin conductance, skin temperature, and more recently the EEG. A representation of these processes can be fed back to the person being monitored in the form of auditory and/or visual stimuli. In biofeedback (BFB) interventions, in addition to monitoring such stimuli, the individual is taught through an operant conditioning paradigm to increase or decrease the amount of a signal reflecting the biological process, with the goal of improving health and/or optimizing performance. BFB approaches have been well documented for basic physiological processes such as headache from excessive muscle tension or cold hands from peripheral vascular constriction. Neurofeedback (NFB) involves monitoring and modifying brainwave activity as detected on the EEG.

\subsection{Benefits of Neurofeedback}

Neurofeedback interventions are currently being applied to altering the usual neuroelectrophysiologic changes seen in normal aging, as well as abnormal processes such as dementia. While initially NFB focused on one brain EEG site, it has since expanded to be used with multiple sites, and in both static and dynamic domains. Doing so, NFB treatment can help reverse the slowing of the alpha rhythm by focusing on one to four brain sites [43, 44]. It also can normalize neuroelectrical activity at multiple sites simultaneously, thereby increasing efficient activation and communication throughout the cerebral cortex. This latter technique focuses training on four to nineteen brain sites and is called Live $Z$ Score Training [44].

There is promising research suggesting neurofeedback can improve cognition in the elderly [45] Neurofeedback to enhance cognitive functioning and to counter the effects of aging has been referred to as "brain brightening" $[46,47]$. Other research suggests a positive correlation between excessive slow brainwave (theta) activity and mild cognitive impairment or dementia [48]. Two studies found neurofeedback training that involved the inhibition of theta activity in participants aged 60-85 led to improved cognitive performances on neuropsychological tests such as verbal comprehension, attention, orienting, recognition memory, and executive functions [49, 50]. Anecdotal evidence suggests that NFB paired with aerobic exercise may further reduce neurocognitive decline in the elderly.

While most traditional physiologic biofeedback interventions have not shown promise for reducing age-related cognitive decline, passive Infrared hemoencephalography (pIR HEG) 
biofeedback using sensors positioned proximally over the forehead and distal from the frontal lobes measure temperature as an indirect measurement of frontal blood flow. Increasing blood flow and frontal temperature is thought to reflect increased activation of the frontal cortices. In normal ageing, there are cellular atrophy and microvascular changes felt to diminish higher level cortical functions, such as executive abilities and working memory [51]. pIR HEG is an non-invasive , easy intervention which may help with these critical functions in normal aging or pathological conditions [52].

\section{Transcranial Neuromodulation Interventions}

Neuromodulation therapies allow focused delivery of modifying agents (e.g. electrical, optical or chemical signals) to targeted areas of the nervous system in order to improve neural function. Neuromodulation differs from traditional neurofeedback by directing stimuli into the brain, rather than passively monitoring the brain's signals, and changing their frequency and/or intensity by operant conditioning and self-regulation strategies.

\subsection{Transcranial Photobiomodulation}

A promising area of clinical research in the prevention of neurocognitive decline is the use transcranial photobiomodulation (aka Low Level Light Therapy or LLLT), which involves the noninvasive application of near infrared (NIR) light (typically $810 \mathrm{~nm}$ ) to a person's head. NIR, at a specific pulse wavelength, has been shown to penetrate the scalp and skull to the cerebral cortex. One wears a headset with four groups of light emitting diodes that focus on hubs of the important Default Mode Network, a network of brain electrical activity for awareness, cognition and consciousness. Photobiomodulation is thought to enhance mitochondrial function (and therefore ATP production) and reduce neuronal apoptosis in human and animal cells [53]. The postulated mechanism of action for photobiomodulation is increased cerebral oxygenation, which serves to activate neuronal repair and growth while reducing inflammation [54].

\subsection{Benefits of Transcranial Photobiomodulation}

There is increasing evidence that transcranial photobiomodulation may be effective in the treatment of neurodegenerative disorders such as Alzheimer's and Parkinson's diseases [54-57]. In addition there is evidence that the application of NIR to the cerebral cortex can improve mood and cognitive functions in healthy subjects, including enhancing reaction time, attention, memory, and executive functions, all areas that normally decline with advancing age [58-61].

\subsection{Other Transcranial Neuromodulation Interventions}

Chemical neuromodulation involves the use of traditional psychopharmacology, which is beyond the scope of this article. Older neuromodulation approaches include electrical and electromagnetic stimulation of the brain through wearable sensors. While this technology has applications for mood disorders such as anxiety and depression, it has not shown efficacy in agerelated neurocognitive decline nor in the dementias. 


\section{Other Approaches to Support Healthy Aging}

\subsection{Sauna Benefits}

The use of saunas for improving overall health has been an area of recent scientific investigation. There is increasing empirical evidence that regular sauna use of at least two to three times per week for 20 minutes at a temperature of $174 \mathrm{~F}$ in conventional saunas (and likely less with infrared saunas) may be associated with reduced overall mortality [62], blood pressure [63], and risk of stroke [64], cardiac disease [62], respiratory diseases [65], and Alzheimer's disease or other causes of dementia [66]. The mechanisms are not completely understood but appear to be related to a reduction in inflammation, blood pressure, insulin resistance, and arterial stiffness in the brain and body [67]. In addition, it is assumed that regular sauna use has effects on hormones such as growth hormone and insulin-like growth factor-1 (IGF-1) which promote muscle and body repair and increase neurogenesis. Heat stress from sauna use also increases BDNF when combined with exercise. BDNF increases the growth of new brain cells, the survival of existing neurons, and neuroplasticity, which may lead to increasing cognitive reserve. There is also evidence that exposure to heat via saunas can have positive effects on psychological health [68]. This is likely due to increased release and storage of norepinephrine, which can improve cognitive functions such as attention, and prolactin found to be associated with myelination.

\subsection{Supplements}

A number of supplements have been studied in hopes to improve health and reduce the effects of aging. The most conservative view regarding supplementation is that supplements are needed only if one is deficient in a certain vitamin or mineral needed for optimal metabolism, and these cannot be obtained through a healthy diet. However, other healthcare providers aggressively recommend supplements. A cautious middle position is that supplements with an adequate evidence base may be useful when used as part of a comprehensive, well monitored healthcare plan. Among the most researched supplements are various precursors to the coenzyme nicotinamide adenine dinucleotide $(\mathrm{NAD}+)$, including components of vitamin $\mathrm{B} 3$ such as niacinamide and nicotinamide riboside. NAD+ has been found to decline with age [69], and associated with various age-related diseases [70]. NAD+ has been found to activate anti-aging enzymes such as sirtuins, enzymes that help repair damaged DNA (e.g., PARPs), improve agerelated decline in the circadian rhythm [71], and improve mitochondrial health [72]. In addition, healthy NAD+ levels may protect the brain against axonal degeneration [73], and improve cognitive function or slow the progression of Alzheimer's disease in animal models [74]. Levels of Vitamins D and B-12 are routinely measured in regular physical examinations, given their contribution to physical and cognitive health.

The purported health benefits of red wine are thought to be primarily the result of the polyphenol antioxidant resveratrol that is found in the vines and skin of red grapes. Resveratrol, also available as a supplement, has been found to improve mitochondrial function in animal models [75, 76], and may play a role in preventing Alzheimer's disease [77]. However, the research is contradictory. The recommendation of alcohol use is also controversial. In a recent editorial in the British Medical Journal, Welch notes memory problems from hippocampal atrophy with 
alcohol use in a dose dependent manner and no health benefit for light drinkers over abstainers [78].

\section{Conclusion}

Normal aging does not inevitably portend acquisition of chronic health problems and faltering mental acuity. As we have attempted to show, avoidance of certain unhealthy habits and behaviors and adoption of healthy habit and behavioural alternatives can foster good mental and neurocognitive health throughout the lifespan. Resistance to societal pressure, aggressive commercial marketing and immediate gratification in favour of healthier alternatives does require some self-discipline or self-regulation, but ultimately yields health rewards. As discussed, mounting research evidence supports identification of Exercise, Sleep and Diet/Nutrition as core health modifiers for healthy living at any age. Following specific cardiovascular exercise guidelines, implementing sleep hygiene strategies to improve restorative sleep, and maintaining a brain and heart healthy diet can forestall or minimize the normal physical and mental decline often associated with aging. Adjunct activities and complementary interventions such as neurofeedback, neuromodulation, sauna and supplements are also being investigated and show some promise for healthy physical and neurocognitive aging. Ironically, despite impressive and expensive advances in neuroimaging and genetics and their contribution to the neuroscientific study of Alzheimer's and dementia, research supports behavioural and basic health strategies as most efficacious in a neuroprotective capacity. Behavioural and complementary interventions, coupled with a positive attitude and a supportive community, may help optimize physical and neurocognitive abilities throughout the lifespan.

\section{Disclaimer}

The information in this article is intended for educational purposes only. One should consult with one's physician or appropriate healthcare provider before initiating any therapies addressed in this article.

\section{Author Contributions}

RLC and AAC conceptualized the paper. RLC, CF and AAC wrote the paper.

\section{Competing Interests}

The authors have declared that no competing interests exist.

\section{References}

1. World's older population grows dramatically [Internet]. National Institutes of Health (NIH). 2016 [cited 2018 November 29]. Available from: https://www.nih.gov/news-events/newsreleases/worlds-older-population-grows-dramatically

2. Sahoo K, Sahoo B, Choudhry A, Sofi N, Kumar, Bhadoria, A. Childhood obesity: Causes and consequences. J Fam Med Primary Care. 2015; 4: 187-192. 
3. Long C. When physical education is cut, who picks up the slack? NEA TODAY. [cited 2019 February 27]. Available from: http://neatoday.org/2017/03/28/cuts-to-physical-education/

4. Stitt C, Kunkel D. Food advertising during children's television programming on broadcast and cable channels. Health Commun. 2008; 23:573-584.

5. Robinson T, Banda J, Hale L, Lu A, Fleming-Milici F, Calvert S, Wartella, E. Screen media exposure and obesity in children and adolescents. Pediatrics. 2017; 140: S97-S101.

6. Obesity Facts [Internet]. Centers for Disease Control and Prevention: CDC Healthy Schools. [Cited February 27, 2019]. Available from: https://www.cdc.gov/healthyschools/obesity/facts.htm

7. Chuang $Y, A n Y$, Bilgei M, Wong D, Troncoso J, O’Brien R, Breitner J, Ferruci L, Resnick S, Thambisetty M. Midlife adiposity predicts earlier onset of Alzheimer's dementia, neuropathology and presymptomatic cerebral amyloid accumulation. Mol Psych. 2016; 21: 910-915.

8. Nine lifestyle changes may reduce risk of dementia [Internet]. nhs.uk. 2017 [cited 2018 November 29]. Available from: https://www.nhs.uk/news/neurology/nine-lifestyle-changesmay-reduce-risk-of-dementia/

9. Baumgart $M$, Synder $H$, Carrillo $M$, Fazio $S$, Kim H, Johns $H$. Summary of the evidence on modifiable risk factors for cognitive decline and dementia: A population-based perspective. Alzheimer's Dementia. 2015; 11: 718-726.

10. Physical activity guidelines for Americans (2nd ed.) 2018. Washington, DC: U.S. Department of Health and Human Services. [Internet]. Available from: https://health.gov/paguidelines/second-edition/]

11. Piercy K, Troiano R, Ballard R, Carlson S, Fulton J, Galuska D, et al. The physical activity guidelines for Americans. JAMA. 2018; 320: 2020.

12. Physical Activity [Internet]. World Health Organization. [Cited 2018 November 29]. Available from: http://www.who.int/dietphysicalactivity/pa/en/

13. Know your target heart rates for exercise, losing weight and health. [Internet]. American Heart Association. [Cited 2019 March 01]. Available from: https://www.heart.org/en/healthyliving/fitness/fitness-basics/target-heart-rates

14. Measuring physical activity. [Internet]. Harvard T. H. Chan School of Public Health. [Cited 2019 March 01]. Available from: https://www.hsph.harvard.edu/nutritionsource/metsactivity-table/

15. Robson J, Janssen I. Intensity of bouted and sporadic physical activity and the metabolic syndrome in adults. PeerJ. 2015; 3: e1437.

16. Top 10 things to know about the second edition of the physical activity guidelines for Americans. [Internet]. Office of Disease Prevention and Health Promotion. [Cited 2019 March 01]. Available from: https://health.gov/paguidelines/second-edition/10things/

17. Segar M, Gibala M. Active voice: From prescription pad to movement menu - Why updated physical activity guidelines for Americans (PAG) are a game-changer. [Internet]. Sports Medicine Bulletin: American College of Sports Medicine. [Cited 2019 February 27]. Available from: http:// http://www.multibriefs.com/briefs/acsm/active022619.htm

18. Colcombe SJ, Erickson KI, Scalf PE, Kim JS, Prakash R, McAuley E, et al. Aerobic exercise training increases brain volume in aging humans. J Gerontol A Biol Sci Med Sci. 2006; 61: 1166-1170. 
19. Warburton D, Nicol C, Bredin S. Health benefits of physical activity: The evidence. CMAJ. 2006; 174: 801-809.

20. Jimenez-Maldonado A, Renteria I, Garcia-Suarez PC, Moncada-Jimenez J, Freire-Royes LF. The impact of high-intensity interval training on brain derived neurotrophic factor in brain: A minireview. Front Neurosci. 2018; 12: 839.

21. Blumenthal J, Babyak M, Moore K, Craighead W, Herman S, Khatri P, et al. Effects of Exercise Training on Older Patients With Major Depression. Arch Intern Med. 1999; 159: 2349-2356.

22. Loprinzi P, Davis R. Bouted and non-bouted moderate-to-vigorous physical activity with health-related quality of life. Prev Med Reps. 2016; 3: 46-48.

23. Mandolesi L, Polverino A, Montuori S, Foti F, Ferraioli G, Sorrentino P, et al. Effects of physical exercise on cognitive functioning and wellbeing: Biological and psychological benefits. Frontiers in Psych. 2018; 8: 1-11.

24. Executive Summary: Physical activity guidelines for Americans (2nd ed.) 2018. [Cited 2019 March 01]. Washington, DC: U.S. Department of Health and Human Services. [Internet]. Available from: https://health.gov/paguidelines/second-edition/pdf/PAG ExecutiveSummary.

25. Pargman D. Boomercise. Morgantown: Fitness Information Technology; 2012.

26. Baniqued $P$, Gallen C, Voss M, Burzynska A, Wong C, Cooke $G$, et al. Brain network modularity predicts exercise-related executive function gains in older adults. Front Neuroendocrinol. 2018; 9.

27. Kramer A, Hahn S, Cohen N, Banich M, McAuley E, Harrison R, et al. Aging, fitness and neurocognitive function. Nature. 1999; 400: 418.

28. Northey JM, Cheruin N, Pumpa KL, Smee DJ, Rattray B. Exercise interventions for cognitive functions in adults older than 50: A systematic review with meta-analysis. Br J Sports Med. 2018; 52: 154-160.

29. Gallaway P, Miyake H, Buchowski M, Shimada M, Yoshitake Y, Kim A, et al. Physical activity; A viable way to reduce risks of mild cognitive impairment, Alzheimer's diease, and vascular dementia in older adults. Brain Sci. 2017; 7: 1-16.

30. Angelakis E, Stathopoulou S, Frymiare J, Green D, Lubar J, Kounios J. EEG Neurofeedback: A brief overview and an example of peak alpha frequency training for cognitive enhancement in the elderly. Clin Neuropsychol. 2007; 21: 110-129.

31. Gibala M, Heisz J, Nelson A. Interval training for cardiometabolic and brain health. ACSM's Health Fit J. 2018; 22: 30-34.

32. Zimmerman M. 99 ways to add healthy years to your life. AARP Bulletin. 2019: 1016.

33. Sanchez-Lopez J, Silva-Pereyra J, Fernández T, Alatorre-Cruz G, Castro-Chavira S, GonzálezLópez $M$, et al. High levels of incidental physical activity are positively associated with cognition and EEG activity in aging. PLOS ONE. 2018; 13: e0191561.

34. Moraes W, Piovezan R, Poyares D, Bittencourt L, Santos-Silva R, Tufik S. Effects of aging on sleep structure throughout adulthood: a population-based study. Sleep Med. 2014; 15: 401409.

35. Alhola P, Polo-Kantola P. Sleep deprivation: Impact on cognitive performance. Neuropsychiatry Dis Treat. 2007; 3: 553-567.

36. Tarasoff-Conway J, Carare R, Osorio R, Glodzik L, Butler T, Fieremans E, et al. Clearance systems in the brain-implications for Alzheimer disease. Nat Rev Neurol. 2015; 11: 457-470. 
37. Adult Obesity Causes \& Consequences | Overweight \& Obesity | CDC [Internet]. Cdc.gov. [updated 2018; cited 2018 November 29]. Available from: https://www.cdc.gov/obesity/adult/causes.html

38. Rainey-Smith S, Gu Y, Gardener S, Doecke J, Villemagne V, Brown B, et al. Mediterranean diet adherence and rate of cerebral $A \beta$-amyloid accumulation: Data from the Australian imaging, biomarkers and lifestyle study of ageing. Transl Psychiatry. 2018; 8: 238.

39. Cohen M. Brain food. Neurol Now. 2017; 13: 22-35.

40. McEvoy C. Healthy Eating Habits May Preserve Cognitive Function and Reduce the Risk of Dementia. Alzheimer's Association International Conference; $16^{\text {th }}-20^{\text {th }}$ July 2017; London, England.

41. Jacka F, Cherbuin N, Anstey K, Sachdev P, Butterworth P. Western diet is associated with a smaller hippocampus: A longitudinal investigation. BMC Med. 2015; 13: 215.

42. Koyama K, Hirasawa $H$, Okubo $Y$, Karasawa A. Quantitative EEG correlates of normal aging in the elderly. Clin EEG Neurosc. 1997; 28: 160-165.

43. Soutar, R. \& Longo, R.E. Doing Neurofeedback: An Introduction. San Rafael: ISNR Research Foundation; 2001.

44. Collura T, Frederick J. Handbook of clinical QEEG and neurotherapy. New York: London Routledge; 2017.

45. Gruzelier J. EEG-neurofeedback for optimising performance. I: A review of cognitive and affective outcome in healthy participants. Neurosci Biobehav Rev. 2014; 44: 124-141.

46. Hammond D. What is neurofeedback: An update. J Neurotherapy. 2011; 15: 305-336.

47. Budzynski, T.H. Brain brightening: Can neurofeedback improve cognitive process? Biofeedback. 1996; 24: 14-17.

48. Babiloni C, Squitti R, Del Percio C, Cassetta E, Ventriglia M, Ferreri F, et al. Free copper and resting temporal EEG rhythms correlate across healthy, mild cognitive impairment, and Alzheimer's disease subjects. Clin Neurophysiol. 2007; 118: 1244-1260.

49. Becerra J, Fernández T, Roca-Stappung M, Díaz-Comas L, Galán L, Bosch J, et al. Neurofeedback in healthy elderly human subjects with electroencephalographic risk for cognitive disorder. J Alzheimers Dis. 2012; 28: 357-367.

50. Wang J, Hsieh S. Neurofeedback training improves attention and working memory performance. Clin Neurophysiol. 2013; 124: 2406-2420.

51. Calso C, Besnard J. Normal aging of frontal lobe functions. Geriatr Psychol Neuropsychiatr Vieil. 2016; 14: 77-85.

52. Pérez-Álvarez F, Timoneda-Gallart C, Serra-Sala M. Clinical usefulness of hemoencephalography beyond the neurofeedback. Neuropsychiatr Dis Treat. 2016: 12: 11731180.

53. Lu Y, Wang R, Dong $\mathrm{Y}$, Tucker $\mathrm{D}$, Zhao N, Ahmed $\mathrm{M}$, et al. Low-level laser therapy for beta amyloid toxicity in rat hippocampus. Neurobiol Aging. 2017; 49: 165-182.

54. Hamblin M. Shining light on the head: Photobiomodulation for brain disorders. BBA Clinical. 2016; 6: 113-124.

55. Lapchak P. Transcranial near-infrared laser therapy applied to promote clinical recovery in acute and chronic neurodegenerative diseases. Expert Rev Med Devices. 2012; 9: 71-83. 
56. Purushothuman S, Johnstone D, Nandasena C, Mitrofanis J, Stone J. Photobiomodulation with near infrared light mitigates Alzheimer's disease-related pathology in cerebral cortex evidence from two transgenic mouse models. Alzheimer's Res Ther. 2014; 6: 2.

57. Johnstone D, Moro C, Stone J, Benabid A, Mitrofanis J. Turning on lights to stop neurodegeneration: The potential of near infrared light therapy in Alzheimer's and Parkinson's disease. Fronti Neurosci. 2016; 9.

58. Blanco N, Maddox W, Gonzalez-Lima F. Improving executive function using transcranial infrared laser stimulation. J Neuropsychol. 2015; 11: 14-25.

59. Disner S, Beevers C, Gonzalez-Lima F. Transcranial laser stimulation as neuroenhancement for attention bias modification in adults with elevated depression symptoms. Brain Stimul. 2016; 9: $780-787$.

60. Grover F, Weston J, Weston M. Acute effects of near infrared light therapy on brain state in healthy subjects as quantified by qEEG measures. Photomed Laser Surg. 2017; 35: 136-141.

61. Gonzalez-Lima F, Barrett D. Augmentation of cognitive brain functions with transcranial lasers. Fronti Systs Neurosci. 2014; 8.

62. Laukkanen $\mathrm{T}$, Khan $\mathrm{H}$, Zaccardi F, Laukkanen J. Association between sauna bathing and fatal cardiovascular and all-cause mortality events. JAMA Intern Med. 2015; 175: 542.

63. Zaccardi F, Laukkanen T, Willeit P, Kunutsor S, Kauhanen J, Laukkanen J. Sauna bathing and incident hypertension: A prospective cohort study. Am J Hypertens. 2017; 30: 1120-1125.

64. Kunutsor S, Khan H, Zaccardi F, Laukkanen T, Willeit P, Laukkanen J. Sauna bathing reduces the risk of stroke in Finnish men and women. Neurol. 2018; 90: e1937-e1944.

65. Kunutsor S, Laukkanen T, Laukkanen J. Sauna bathing reduces the risk of respiratory diseases: a long-term prospective cohort study. Eur J Epidemiol. 2017; 32: 1107-1111.

66. Laukkanen T, Kauhanen J, Laukkanen J. Sauna bathing is inversely associated with dementia and Alzheimer's disease in middle-aged Finnish men. Age Ageing. 2017; 46: 245-249.

67. Laukkanen J, Laukkanen T. Sauna bathing and systemic inflammation. Eur J Epidemiol. 2017; 33: 351-353.

68. Janssen C, Lowry C, Mehl M, Allen J, Kelly K, Gartner D, et al. Whole-body hyperthermia for the treatment of major depressive disorder. JAMA Psychiatry. 2016; 73: 789.

69. Massudi H, Grant R, Braidy N, Guest J, Farnsworth B, Guillemin G. Age-associated changes in oxidative stress and NAD+ metabolism in human tissue. PLoS ONE. 2012; 7: e42357.

70. Houtkooper R, Auwerx J. Exploring the therapeutic space around NAD+. J Cell Biol. 2012; 199: 205-209.

71. Nakahata Y, Sahar S, Astarita G, Kaluzova M, Sassone-Corsi P. Circadian control of the NAD+ salvage pathway by CLOCK-SIRT1. Science. 2009; 324: 654-657.

72. Imai S, Guarente L. NAD+ and sirtuins in aging and disease. Trends Cell Biol. 2014; 24: 464-471.

73. Sasaki Y, Araki T, Milbrandt J. Stimulation of nicotinamide adenine dinucleotide biosynthetic pathways delays axonal degeneration after axotomy. J Neurosci. 2006; 26: 8484-8491.

74. Gong B, Pan Y, Vempati P, Zhao W, Knable L, Ho L, et al. Nicotinamide riboside restores cognition through an upregulation of proliferator-activated receptor- $\nu$ coactivator $1 \alpha$ regulated $\beta$-secretase 1 degradation and mitochondrial gene expression in Alzheimer's mouse models. Neurobiol Aging. 2013; 34: 1581-1588. 
75. Csiszar A, Labinskyy N, Pinto J, Ballabh P, Zhang H, Losonczy G, et al. Resveratrol induces mitochondrial biogenesis in endothelial cells. Am J Physiol Heart Circ Physiol. 2009; 297: H13H2O.

76. Kim S, Joe $Y$, Zheng $M, \operatorname{Kim~H}, \mathrm{Yu} J$, Cho G, et al. Resveratrol induces hepatic mitochondrial biogenesis through the sequential activation of nitric oxide and carbon monoxide production. Antioxid Redox Signal. 2014; 20: 2589-2605.

77. Porquet D, Casadesús G, Bayod S, Vicente A, Canudas A, Vilaplana J, et al. Dietary resveratrol prevents Alzheimer's markers and increases life span in SAMP8. AGE. 2012; 35: 1851-1865.

78. Welch K. Alcohol consumption and brain health. BMJ. 2017; 357: j2645.

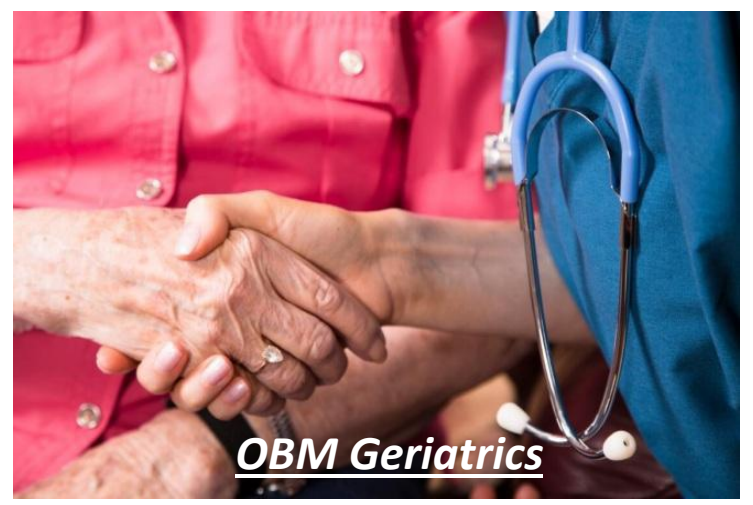

Enjoy OBM Geriatrics by:

1. Submitting a manuscript

2. Joining in volunteer reviewer bank

3. Joining Editorial Board

4. Guest editing a special issue

For more details, please visit:

http://www.lidsen.com/journals/geriatrics 
Original Research

\title{
Prediction of ADRs and Estimation of Polypharmacy in Older Patient's Population: Retrospective Study in Russian Gerontology Center
}

Ali Al-Ragawi ${ }^{1,2}$, Sergey Zyryanov ${ }^{1,3,{ }^{*}}$, Elena Ushkalova ${ }^{1}$, Olga Butranova ${ }^{1}$, Anton Pereverzev ${ }^{4, \neq}$

1. Department of General and Clinical Pharmacology, People's Friendship University of Russia (PFUR), Moscow, Russia; E-Mails: alirajwi4075@gmail.com; sergey.k.zyryanov@gmail.com; eushk@yandex.ru; butranovaolga@mail.ru

2. Department of Pharmacology, Taiz University, Taiz, Yemen;

3. Multifield City Clinical Hospital № 24, Moscow, Russia;

4. Pirogov National Research Medical University, Moscow, Russia; E-Mail: acchirurg@mail.ru

$¥$ Current Affiliation: Clinical and Research Centre of Gerontology, Moscow, Russia

* Correspondence: Sergey Zyryanov; E-Mail: sergey.k.zyryanov@gmail.com

Academic Editor: Michael Fossel

OBM Geriatrics

2019, volume 3 , issue 1

doi:10.21926/obm.geriatr.1901038
Received: October 10, 2018

Accepted: February 25, 2019

Published: March 05, 2019

\begin{abstract}
Background: Adverse drug reactions (ADRs) are common in the elderly and often have serious clinical and economic consequences. Prediction of ADRs is a valuable tool to improve pharmacotherapy and outcomes in this category of patients. Objective: Prediction of ADRs in patients $\geq 65$ years old, based on GerontoNet risk score with emphasis on polypharmacy.

Methods: Pharmacoepidemiological retrospective study of medical records of patients admitted to the Russian Gerontology Clinical Research Center (RGCRC) between 1 June and 30 December $2017(n=201)$. The GerontoNet ADR risk score was calculated for all patients. We used $\chi 2$ tests and t-test to compare categorical variables and analyses of variance (ANOVA) to compare continuous variables. Receiver operator characteristic (ROC) curves were constructed to test the ability of the GerontoNet risk score to predict ADRs. Polypharmacy parameters before admission and during hospital stay were analyzed.
\end{abstract}

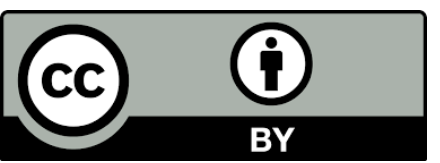

(C) 2019 by the author. This is an open access article distributed under the conditions of the Creative Commons by Attribution License, which permits unrestricted use, distribution, and reproduction in any medium or format, provided the original work is correctly cited. 
Results: The study included 201 medical records of elderly patients. The mean age ( \pm SD) was $76.1 \pm 6.2$ years, $74.6 \%$ of patients were female and the median number of drugs per patient was 8 (range 6-11). Before hospital admission, exposure to ADRs was reported in 46 patients (22.9\%). GerontoNet risk score before admission (mean \pm SD) was $2.54 \pm 1.4 ; 111$ patients $(55.2 \%)$ were with $0-2$ points and $90(44.8 \%)$ with $\geqslant 3$ points. GerontoNet risk score during hospital stay was $4.79 \pm 2.1$. Score was higher in patients with polypharmacy ( $\geqslant 5$ drugs), compared with those without. The area under the curve (ROC) was 0.887 ( $95 \% \mathrm{Cl}$ : 0.84-0.94), the cut-off point of 4 indicated a very good sensitivity and good specificity of results. Before admission, polypharmacy was seen in $36.3 \%$ of patients whereas during hospital stay - in $91 \%$. Polypharmacy exposure increased during hospital stay $(P<0.001)$; consequently, GerontoNet risk score was increased. Variables contributing to ADR risk score include renal failure, polypharmacy, age $\geq 75$ years and the presence of comorbidities. During hospital stay patients with a high GerontoNet risk score $(\geq 4)$ were predicted to be exposed to ADRs at least four times more frequently than patients with low GerontoNet risk score (03) (OR: 15.08; 95\%Cl 7.3-31.15; OR: 3.103; 95\% Cl, 0.984-9.785).

Conclusions: High prevalence of multi-morbidity among the elderly is one of the leading factors of polypharmacy for this population. Adverse reactions in older people can be assessed using GerontoNet, although the GerontoNet ADR risk score improperly classified $21.9 \%$ of patients as high-risk group. Review of appropriateness of medication use is essential, as polypharmacy enhance risk of adverse effects in the elderly population.

\section{Keywords}

Adverse drug reaction (ADR); elderly; GerontoNet ADR risk score; polypharmacy

\section{Introduction}

The elderly population has a marked trend to be increased worldwide; it is supposed to grow from about $9 \%$ (2018) up to $16 \%$ and more in mid-century [1]. The same population aging trends are seen in the Russian Federation - an increase in the population of elderly people in absolute number and proportion [2].

Increase in proportion of people over 65 means increase in a number of patients living with illness and disability. There is an increase of total morbidity rate in the elderly: in the Russian Federation in 2013 total number of morbidity cases was 208,228.9 per 100,000 of people, that is 1.3 times higher than the rate in general population of a country (161,061.8 people per 100 thousand people). According to the data of clinical studies, about $80 \%$ of elderly patients has at least one chronic disease and $55 \%$ to $98 \%$ patients are affected by multi-morbidity (co-existence of a number of chronic diseases in the same individual) [3]. Data from population-based longitudinal study (3 years) revealed that $33.6 \%$ of participants with no chronic disease and $66.4 \%$ of those with at least one disease at baseline developed multi-morbidity: the incidence rate was 12.6 per 100 person-years (95\% Cl: 9.2-16.7) and 32.9 per 100 person-years (95\% Cl: 28.1-38.3), respectively [4]. A cross-sectional study of 1.7 million patients registered in the primary care in Scotland showed the level of multi-morbidity at $81.5 \%$ for individuals over 85 [5]. 
The combination of acute and chronic pathology in elderly category of patients results in a strong tendency for polypharmacy. Scottish study revealed great increase of population over 65 with polypharmacy, with $16.4 \%$ receiving 10 or more medications [6]. The data from the systematic review performed by Storms $\mathrm{H}$. et al (2017) revealed next prevalence of inappropriate medication use in the elderly: $18.5 \%$ to $82.6 \%$ (median $46.5 \%$ ) when Beers criteria in general ('B1991,' 'B1997,' 'B2003' and 'B2012') were used; 21.3\% to 63.0\% (median 35.1\%) when inappropriate medication use was assessed only with the complete criteria list of Beers 2003 ('B2003,' both criteria independent of disease 'ID' and criteria considering disease 'CD'); $63.0 \%$ to $82.6 \%$ in studies used 2012 update of the Beers criteria ('B2012'); 23.7\% to 79.8\% (median 61.1\%)) for studies used STOPP and from $30.5 \%$ up to $74.0 \%$ (median $48.6 \%$ ) for studies used START [7]. The prevalence of multi-morbidity, polypharmacy, and inappropriate medication use in the elderly results in a dramatic increase of ADR risk, also worsened by pharmacokinetic and pharmacodynamic age-related changes. Investigations show that up to $60 \%$ of nursing home residents experience ADRs [8]. 10\% median ADR-related hospital admission rate for patients $\geqslant 65$ was revealed in the systematic review performed by Kongkaew C. et al (2008) [9] and $11 \%$ rate in the systematic review of Alhawassi TM et al (2014) [10]. Polypharmacy is one of the leading risk factors for ADR: there is a $13 \%$ increased risk of ADRs in the case of two drugs administration, 58\% in the case of five drugs and $82 \%$ in case of seven or more drugs [11]. Beijer H.J. et al (2002) demonstrated that about $88 \%$ of the ADRs related to hospitalizations are preventable in the elderly, compared with $24 \%$ in the younger patients [12]. Thus, the tool to predict ADR risk in the elderly is highly desirable for real clinical practice, though many attempts were made with uncertain results.

The GerontoNet ADR risk score has been developed based on the analytic work of the Italian Group of Pharmacoepidemiology in the Elderly (GIFA) (5936 older inpatients), a validation study that included 483 patients (mean (+SD) age was $80.3 \pm 7.6$ years) with the area under the receiver operator characteristic curve 0.70 (95\% confidence interval, 0.63-0.78) [13]. A recent validation of this tool was performed in the study of Mirko Petrovic et al (2017) based on the data from the prospective CRIteria to assess appropriate Medication use among Elderly complex patients (CRIME) cohort [14]. The GerontoNet ADR risk score ranges from 0 to 10 points and includes six variables, with each scoring based on the strength of its association with ADRs: presence of 4 or more comorbid conditions ( 1 point), renal failure (1 point), heart failure (1 point), liver disease (1 point), number of drugs ( 1 point when between 5 and 7; 4 points when 8 or more) and a history of ADRs (2 points).

Objective of the study: prediction of ADRs in patients $\geqslant 65$ years old, based on GerontoNet risk score with emphasis on polypharmacy.

\section{Materials and Methods}

\subsection{Study Design}

Retrospective Pharmacoepidemiologic study. Study was approved by the Ethic Committee of Medical Institute of People's Friendship University of Russia, (Protocol No. 9 from 17/05/2018). 


\subsection{Study Setting and Population}

The records of patients $\geqslant 65$ years old discharged between $1^{\text {st }}$ of June and $30^{\text {th }}$ of December 2016 from Russian Gerontology Clinical Research Center (RGCRC) in Moscow were studied. Inclusion criteria: $\geqslant 65$ years old, administration of at least 1 drug. The final study included 201 records. Analyzed data: demographics, reasons for admission, comorbidities, diagnosis at admission and at discharge, baseline laboratory data, medications taken before admission and during hospital stay, and medications prescribed at discharge. Data were collected on a standardized data collection form (Microsoft Excel ${ }^{\circledR}$ 2016). All patient' s records were subdivided into 3 age groups ( $65-74,75-84$ and $\geqslant 85$ year). Estimated creatinine clearance rate (eCCr) was evaluated using Cockcroft-Gault formula. The state law on personal data protection was fulfilled. We defined polypharmacy as use of $\geqslant 5$ drugs, and hyperpolypharmacy as use of 8 or more drugs, based on the results of systematic review made by Masnoon N. et al (2017) [15]. The GerontoNet $A D R$ risk score was calculated for all patients. Receiver operator characteristic (ROC) curve was constructed to test the ability of the GerontoNet risk score to predict ADRs.

\subsection{Statistical Analysis}

Patients data were stored in Excel 2016 (Microsoft ${ }^{\circledR}$, USA) and exported to SPSS ver. 22 (IBM ${ }^{\circledR}$, USA) for statistical analysis. Descriptive statistics were generated to describe the characteristics of the study population. We used $\chi 2$ tests and $t$ tests to compare categorical variables and analyses of variance (ANOVA) to compare continuous variables. Receiver operator characteristic (ROC) curves were constructed, and the area under the curve (AUC) was calculated to determine the predictive ability of the GerontoNet ADR risk score. Sensitivity and specificity were calculated for a cut-off point of GerontoNet score before admission and during hospital stay.

\section{Results}

\subsection{Patient Characteristics}

We studied medical records of 201 patients. $56.7 \%$ of patients were admitted in the cardiac ward, $32.4 \%$ in the therapeutics and $10.9 \%$ in neurology. The patients mean age ( \pm SD) was 76.1 \pm 6.3 years ( $\min -65$, $\max -94$ ); $74.6 \%$ patients were female, $85.1 \%$ of patients had at least four chronic comorbidities: hypertension, ischaemic heart disease, cerebrovascular diseases and atherosclerosis of cerebral vessels. In 143 patients (71.1\%), eGFR was $\leq 60 \mathrm{ml} / \mathrm{min} / 1.73 \mathrm{~m} 2 ; 13$ patients (6.5\%) had heart failure class III-IV (based on New York Heart Association (NYHA) classification); 7 patients (3.5\%) had a diagnosis of hepatic failure; 146 patients (72.6\%) had ischemic heart disease (IHD); 96 patients (47.8\%) had heart failure; 123 patients (61.2\%) had arterial hypertension and 44 patients $(21.9 \%)$ had cardiac arrhythmias.

The mean $( \pm S D$ ) number of medications per patient during hospital stay was $8.5 \pm 3.2$ (min -6 , max - 11). The most commonly used medications were Bisoprolol, Amlodipine, Atorvastatin, Losartan, Lisinopril, Enalapril, Indapamide, Piracetam, Benzodiazepines, Acetylsalicylic acid, Furosemide and laxatives. Polypharmacy at admission was observed in 66 patients (32.8\%), which resulted in the increase of GerontoNet risk score by 4 points. Hyperpolypharmacy was seen in 7 patients (3.5\%), which resulted in the increase of GerontoNet risk score by 1 point. 
ADRs were observed in 46 patients $(22.9 \%)$ before admission. Characteristics of the study population are shown in the Table 1.

Table 1 Characteristics of the study population.

Patient demographics and medications

Departments, $\mathrm{n}(\%)$

Cardiac department

Therapy department

Neurology department

Age groups:

$65-74$

$75-84$

$\geqslant 85$

Female

Number of patients with at least 4 comorbidities

Structure of comorbidities:

Ischemic heart disease

Heart failure

Arterial hypertension

Cardiac arrhythmia

Renal failure

Liver disease

Polypharmacy before admission:

Patients used 5-7 drugs

Patients used $\geqslant 8$ drugs

Polypharmacy during hospital stay:

Patients using 5-7 drugs

Patients using $\geqslant 8$ drugs

Number of patients previously reported ADRs

Number of patients with ADRs during hospital stay
$\%$

(Total $n=201$ )

114

65

22

56.2

32.3

10.9

39.8

50.2

10

150

74.6

171

85.1

146

72.6

13

6.5

123

61.2

44

21.9

143

7

71.1

3.5

66

32.8

7

3.5

62

30.9

121

60.1

46

22.9

45.3

ADR, Adverse drug Reaction; SD, standard deviation. 
GerontoNet risk score (Mean $\pm \mathrm{SE}$ ) was $2.54 \pm 1.4$ before admission and $4.79 \pm 2.1$ during hospital stay. Before admission the number of drugs and previous ADRs were the strongest predictors of GerontoNet ADR score $\geqslant 4$ ( $P<0.001$ and $P<0.001$ respectively), and presence of four or more comorbidities was considered as a predictor of $A D R$ score $\geqslant 4$ with $p=0.031$. The same was observed for the hospital stay period, with the number of drugs and previous ADRs being the strongest predictors, $P<0.001$. Before admission, $111(55.2 \%)$ of patients were with $0-2$ points and $90(44.8 \%)$ patients were with $\geqslant 3$ points of GerontoNet risk score. The prevalence of polypharmacy was high during hospital stay where $30.9 \%$, patients used 5-7 medications, and $60.1 \%$ used $\geq 8$ medications (Figure 1 ).

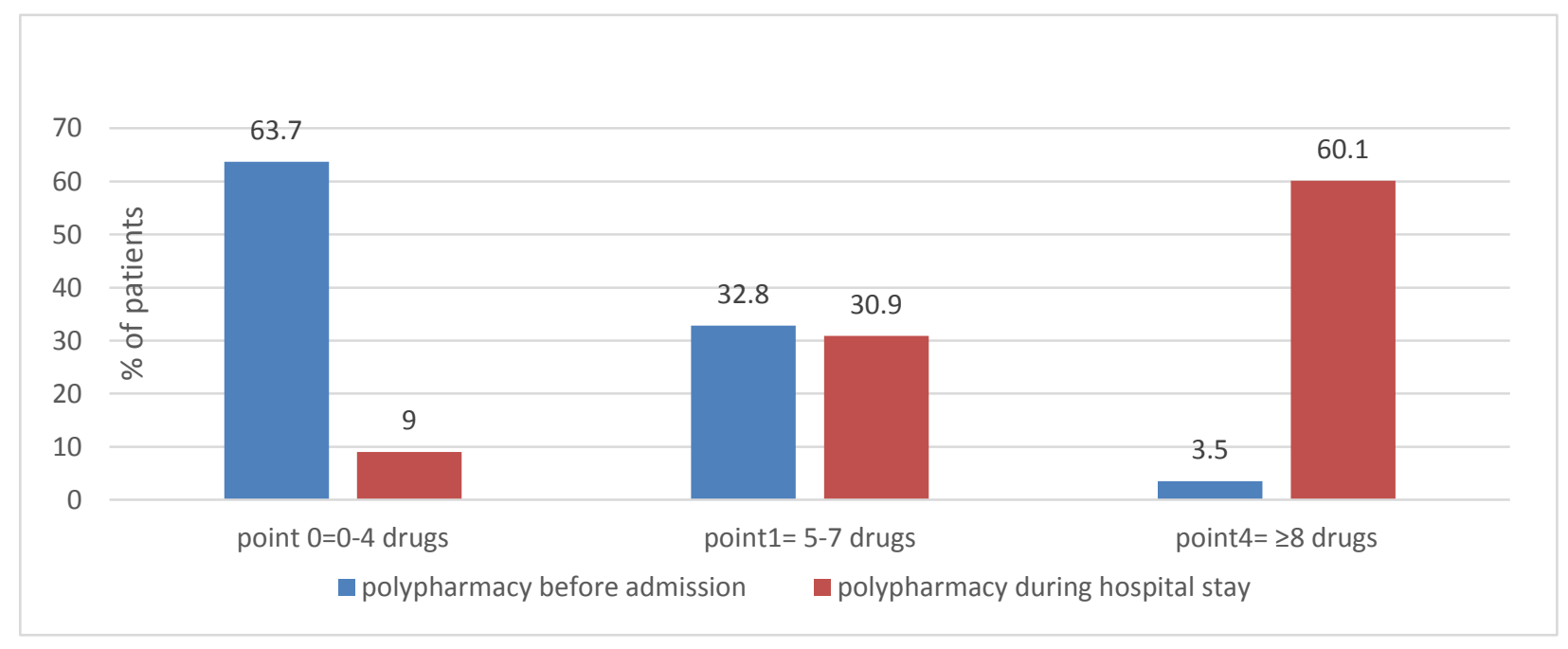

Figure 1 Polypharmacy before admission and during hospital stay.

\subsection{GerontoNet Risk Score and Determination of Cut-Off Point}

The mean (95\% CL) GerontoNet ADR risk score before admission was 2.54 (2.35-2.74) points, range (0-7). It was higher in patients with polypharmacy compared with those without $(29.8 \%$ and $15 \%$, respectively).

ROC analysis was used to evaluate the diagnostic accuracy of the GerontoNet ADR risk score (AUC of 0.887 (95\% CL: 0.84-0.94)) as a tool to predict drug-related ADRs. GerontoNet ADR risk score showed a very good sensitivity $(82 \%)$ and good specificity $(80 \%)$ in population with score $\geqslant$ 3 , so it promised a proper identification of patients who are at high risk for ADRs (Figure 2). The median (IQR) GerontoNet risk score in patients with ADRs was 3.91 (3.66-4.16), and in patient without ADRs was 2.14(1.93-2.34), $P=0.000$. GerontoNet risk score increased with polypharmacy exposure and comorbidity presence (at least 4 diseases), but there was no association between increased GerontoNet risk score and previous ADRs $(P=0.513)$.

The mean GerontoNet ADR risk score during hospital stay ( $95 \% \mathrm{Cl}$ ) was 4.79 (4.51-5.08), range (0-9). It was higher in patients with polypharmacy compared with those without $(66.2 \%$ and $24.9 \%$, respectively). Gender differences were detected: mean (CI 95\%) GerontoNet score in women was greater than in men (2.67 (2.45-2.90) vs $2.16(1.76-2.56), P=0.023)$. The number of patients with polypharmacy and hyperpolypharmacy was the highest in the $75-84$ and $\geqslant 85$-year age groups (29\% and $18.4 \%$, respectively). Polypharmacy was not associated with heart failure $(P=0.448)$ or liver disease $(P=0.716)$. 


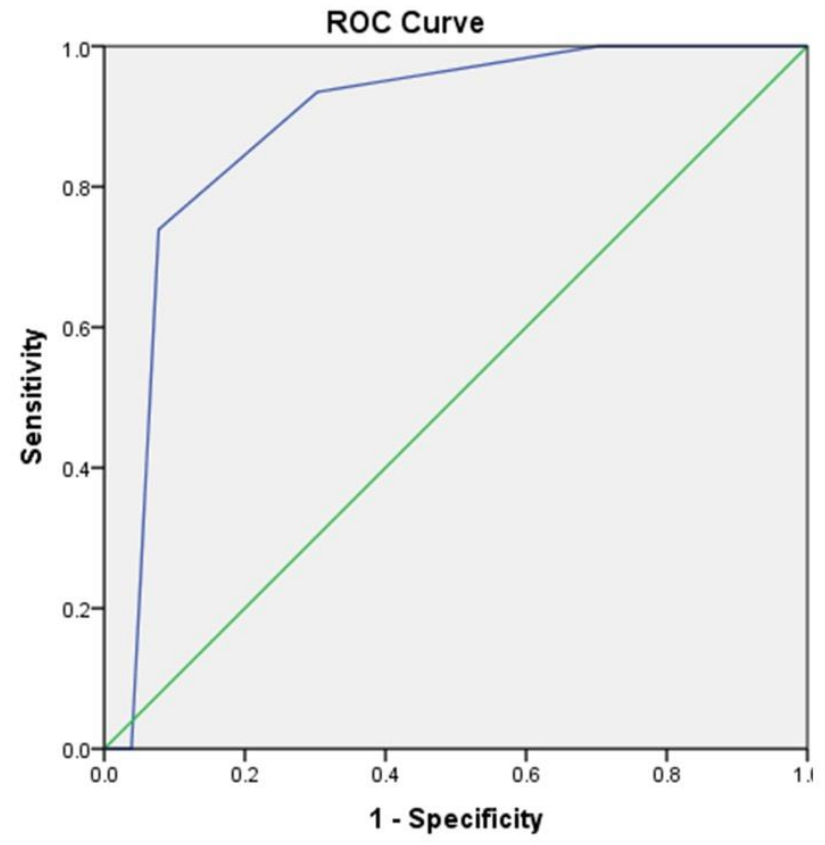

Diagonal segments are produced by ties. i

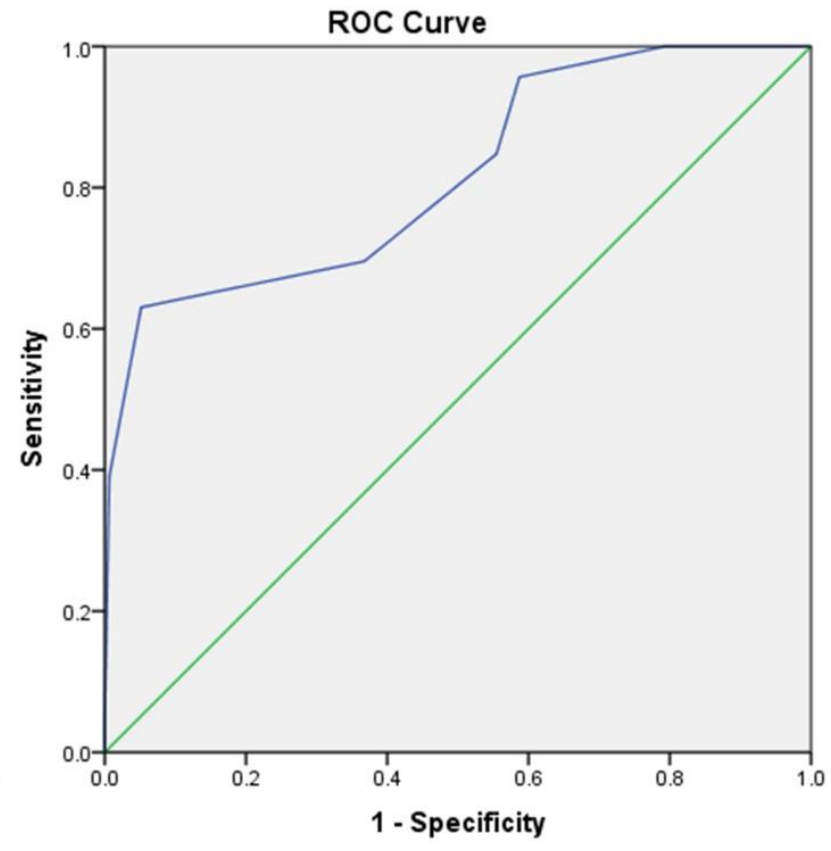

Diagonal segments are produced by ties.

ii

Figure 2 Receiver Operator Curve of GerontoNet ADR risk score and ADRs (i) on admission and (ii) during hospital stay. Receiver operator characteristic (ROC) curve was constructed to test the ability of the GerontoNet risk score to predict ADRs.

\subsection{Adverse Drug Reactions}

Forty-six patients (22.9\%) experienced ADR before hospital stay; of them, $82.6 \%$ had renal failure, $89.1 \%$ were female, $93.5 \%$ had polypharmacy during hospital stay period, and $80.4 \%$ had at least four comorbidities. Patients with ADRs were compared with patients without ADRs. Median age (77 versus 76 years, $P=0.723$ ) was of no difference and GerontoNet risk score was strongly associated with ADRs $(P=0.000)$. Patients with previous ADRs $(n=38)$ had renal impairment (26.6\%); $P=0.051$ ) more typically. Number of daily prescribed drugs during hospital stay per patient was of no difference $(8.31 \pm 3.21$ versus $9.22 \pm 3.01, P=0.089)$. There was a difference between these subgroups in terms of gender $P=0.010$, and no differences in terms of burden of comorbidity, heart failure, polypharmacy before admission and liver disease (Table 2).

We compared patients with and without polypharmacy during hospitalization. Subjects with polypharmacy were older; had higher GerontoNet ADRs risk score before admission and during hospital stay; and had higher number of used medications. Conversely, there were no statistically significant differences between these two groups in gender or previous ADRs exposure. Subjects with polypharmacy had chronic heart failure and liver disease more frequently, although it was statistically insignificant ( $P>0.381, P>0.122$, respectively). The rate of renal failure was higher in patients with polypharmacy compared with patients without polypharmacy $(66.9 \%$ vs $4.5 \%, P \leqslant$ 0.038). Relations between polypharmacy and predictors of ADRs are shown in the Table 3.

Effects of age and gender on GerontoNet ADRS risk score and parameter of polypharmacy before admission were tested with a 2-way ANOVA analysis. Table 4 shows that advanced age and gender had no significant effect. 
Table 2 Predictors of ADRs before admission. Comparison of descriptive characteristics in patients with and without ADRs.

\begin{tabular}{|c|c|c|c|}
\hline Patients characteristics & $\begin{array}{l}\text { Patients with ADRs } \\
(n=46) \\
22.9 \%\end{array}$ & $\begin{array}{l}\text { Patients without } \\
\text { ADRs } \\
(n=155) \\
77.1 \%\end{array}$ & $P$ value \\
\hline Female $\mathrm{n}(\%)$ & $41(89.1)$ & $109(70.3)$ & 0.01 \\
\hline \multicolumn{4}{|l|}{ Age (years) } \\
\hline Median (IQR) & 77 & 76 & 0.723 \\
\hline $65-74(\%)$ & 18(39.1) & $62(40)$ & \\
\hline $75-84(\%)$ & $22(47.8)$ & $79(51)$ & \\
\hline$\geqslant 85(\%)$ & $6(13.1)$ & $14(9)$ & \\
\hline \multicolumn{4}{|l|}{ GerontoNet (before admission) } \\
\hline $0-2$ & $3(6.5)$ & 108(69.7) & 0.001 \\
\hline$\geqslant 3$ & 43(93.5) & $47(30.3)$ & \\
\hline Renal failure (\%) & $38(82.6)$ & $105(67.6)$ & 0.051 \\
\hline Liver disease (\%) & $0(0)$ & $7(4.5)$ & 0.142 \\
\hline Heart failure (\%) & $2(4.3)$ & $11(7.1)$ & 0.506 \\
\hline At least four co-morbidities (\%) & $37(80.4)$ & $134(86.5)$ & 0.315 \\
\hline \multicolumn{4}{|l|}{ Polypharmacy preadmission } \\
\hline $\begin{array}{l}0 \text { point } \\
1 \text { point }\end{array}$ & $17(37)$ & $\begin{array}{l}99(63.9) \\
49(31.6)\end{array}$ & 0.303 \\
\hline 4 points & 0 & $7(4.5)$ & \\
\hline \multicolumn{4}{|l|}{ No. of drugs at admission } \\
\hline Mean \pm SE & $8.31 \pm 3.21$ & $9.22 \pm 3.01$ & \\
\hline 0 point & $3(6.5)$ & $15(9.7)$ & 0.089 \\
\hline 1 point & $11(23.9)$ & $51(32.9)$ & 0.334 \\
\hline 4 points & $32(69.6)$ & $89(57.4)$ & \\
\hline \multicolumn{4}{|l|}{ GerontoNet at discharge } \\
\hline $0-3$ & $2(4.3)$ & $64(41.3)$ & 0.001 \\
\hline$\geqslant 4$ & $44(95.7)$ & $91(58.7)$ & \\
\hline
\end{tabular}


Table 3 Relations between polypharmacy and predictors of ADRs.

\begin{tabular}{|c|c|c|c|}
\hline Predictors of ADRs & $\begin{array}{l}\text { Patients without } \\
\text { polypharmacy } \\
(\mathrm{n}, \%)\end{array}$ & $\begin{array}{l}\text { Patients with } \\
\text { polypharmacy } \\
(\mathrm{n}, \%)\end{array}$ & $P$ value \\
\hline $\begin{array}{l}\text { Gender } \\
\text { Female } \\
\text { Male } \\
\end{array}$ & $\begin{array}{l}11(5.5) \\
7(3.5)\end{array}$ & $\begin{array}{l}139(69.2) \\
44(21.9) \\
\end{array}$ & 0.253 \\
\hline Comorbidities ( $\geqslant 4$ diseases) & $12(6)$ & 159 (79.1) & 0.034 \\
\hline NYHA III-IV & $0(0)$ & $13(6.5)$ & 0.381 \\
\hline Liver disease & $2(1)$ & $5(2.5)$ & 0.122 \\
\hline $\begin{array}{l}\text { GerontoNet score before admission } \\
0-2 \\
\geqslant 3\end{array}$ & $\begin{array}{l}14(7) \\
4(2) \\
\end{array}$ & $\begin{array}{l}97(48.4) \\
86(43) \\
\end{array}$ & 0.002 \\
\hline $\begin{array}{l}\text { Polypharmacy before admission } \\
0-4 \\
5-7 \\
\geqslant 8\end{array}$ & $\begin{array}{l}18(9) \\
0(0) \\
0(0) \\
\end{array}$ & $\begin{array}{l}110(54.7) \\
66(32.8) \\
7(3.5) \\
\end{array}$ & $0.001 *$ \\
\hline $\begin{array}{l}\text { GerontoNet score during hospital stay } \\
0-3 \\
\geqslant 4\end{array}$ & $\begin{array}{l}14(7) \\
4(2) \\
\end{array}$ & $\begin{array}{l}18(9) \\
146(73) \\
\end{array}$ & 0.000 \\
\hline Previous ADRs & $3(1.5)$ & $43(21.4)$ & 0.513 \\
\hline Renal failure & $9(4.5)$ & $134(66.9)$ & 0.038 \\
\hline $\begin{array}{l}\text { Age } \\
65-74) \\
(75-84) \\
\geqslant 85\end{array}$ & $\begin{array}{l}12(6) \\
6(3) \\
0(0)\end{array}$ & $\begin{array}{l}68(33.9) \\
95(47.5) \\
20(10)\end{array}$ & $0.01^{*}$ \\
\hline
\end{tabular}

Statistic analysis: Chi-square test of Independence for categorical variables and ${ }^{*}$ Pearson correlation for continuous variables if Normally distributes. Spearmann correlation if variables Not Normally distributed.

Three age groups were compared by the parameters of GerontoNet risk score, polypharmacy before admission and the number of drugs used. There was no significant difference between these age groups. However, patients $\geqslant 85$ had the highest GerontoNet score (2.95 (2.26-3.64)), more likely to have had polypharmacy before admission $(0.7(0.268-1.132))$, and had used more drugs (9.10 (7.97-10.23)).

There was no significant difference between age subgroups in gender. Mean GerontoNet risk score in women was greater than in men ( $C L$ 95\%: 2.67 (2.45-2.90) and 2.16 (1.76-2.56), respectively). 
Table 4 Effect of age on GerontoNet Risk Score and predictors of ADR for men and women.

indicator Overall Mean

$\begin{array}{ll}(95 \% \mathrm{Cl}) \mathrm{n}= & \text { Age group } \\ 201 & \text { mean }(\underline{\mathrm{SD}})(95 \% \mathrm{Cl})\end{array}$

$\begin{array}{lll}65-74 & 75-84 & \text { Aged } \geqslant 85 \\ (n=80) & (n=101) & (n=20)\end{array} \quad P$ value*

GerontoNet score

$\begin{array}{llrrrr}\text { Total } & 2.54(2.35- & 2.33(2.0- & 2.63(2.37- & 2.95(2.26- & 0.133^{*} \\ \text { Men } & 2.74) & 2.65) & 2.90) & 3.64) & \\ & 2.16(1.76- & 1.8 \pm 1.29 & 2.55 \pm 1.47 & 2.25 \pm 1.71 & 0.551^{* *} \\ \text { Women } & 2.56) & & & & \\ & 2.67(2.45- & 2.56 \pm 1.49 & 2.66 \pm 1.29 & 3.13 \pm 1.41 & 0.356^{* *} \\ & 2.90) & & & & \end{array}$

No. of daily used drugs per patient

\begin{tabular}{lllrrr} 
Total & $8.52(8.07-$ & $8.13(7.38-$ & $8.71(8.09-$ & $9.10(7.97-$ & $0.324^{*}$ \\
& $8.96)$ & $8.87)$ & $9.34)$ & $10.23)$ & \\
Men & $8.43(7.42-$ & $7.88 \pm 4.24$ & $9.23 \pm 2.98$ & $7.5 \pm 1.73$ & $0.390^{* *}$ \\
& $9.45)$ & & & & \\
\multirow{2}{*}{ Women } & $8.55(8.06-$ & $8.24 \pm 2.90$ & $8.57 \pm 3.23$ & $9.5 \pm 2.42$ & $0.343^{* *}$ \\
& $8.04)$ & & & &
\end{tabular}

Polypharmacy before admission

$\begin{array}{llrrrr}\text { Total } & 0.478(0.30- & 0.351(0.187- & 0.515(0.346- & 0.7(0.268- & 0.166^{*} \\ \text { Men } & 0.65) & 0.513) & 0.684) & 1.132) & \\ & 0.387(0.076- & 0.322 \pm 0.37 & 0.5 \pm 0.91 & 0.50 \pm 0.58 & 0.638^{* *} \\ \text { Women } & 0.698) & & & & \\ & 0.568(0.404- & 0.44 \pm 0.83 & 0.519 \pm 0.85 & 0.75 \pm 1.0 & 0.302 * * \\ & 0.733) & & & & \end{array}$

$95 \% \mathrm{Cl}$, Confidence Interval. *univariate analysis, ${ }^{* *}$ Kruskal-Wallis test

\section{Discussion}

Our study revealed polypharmacy increase with advanced age, (from $33.9 \%$ at 65 years old to 47.5\% at 75 years old). Publicized studies also report high polypharmacy prevalence among the elderly population $[15,16]$. The most common reason for simultaneous prescription of several drugs in this category of patients is the presence of comorbidities. In our study, elderly patients were highly exposed to polypharmacy during hospital stay. There was no significant effect of gender or previous ADRs on parameters of GerontoNet ADR risk score and polypharmacy in our study; this finding is consistent with the results of an Australian study [16].

Notably, we identified an association between ADRs and gender. Additionally, patients with comorbidities and polypharmacy before admission were more likely to be exposed to ADRs 
independent of other factors. For patients with ADRs during hospital stay, the results of our study were similar with published results of other authors [16]. Unfortunately, there is no ultimate definition of polypharmacy in the medical society. To operationalize this definition, researchers choose various cut-off points.

Our results revealed GerontoNet score $\geqslant 4$ (a cut-off point to predict ADRs) in $67.2 \%$ of patients. The use of GerontoNet Risk Score in our study resulted in improper classification of $21.9 \%$ of patients as high-risk group. It may be the result of hyperpolypharmacy ( $>7$ drugs) in the studied population, which gave an increase of GerontoNet risk score by 4 points.

In our study, GerontoNet score showed good sensitivity and specificity, so it can be used as a tool to identify patients at high risk for ADRs. According to our data, GerontoNet risk score during hospital stay was greater than that before admission and hence, its predictable value is higher for hospital stay period. Potentially, inappropriate prescribing is a common problem for elderly patients in hospitals. The use of a tool to predict ADRs can reduce polypharmacy, improve outcomes in hospitalized elderly patients and decrease therapy costs [17, 18]. G. Onder et al. [13] revealed that serious ADRs in the elderly occur in $64 \%$ of the cases. In the presence of eight or more GerontoNet risk score points, ADRs develop in $21.7 \%$, and the number of simultaneously administered drugs is the strongest risk factor for the development of the ADRs. GerontoNet risk score in a validation study of 483 patients results in correct prediction of ADRs in $71 \%$ of cases (95\% $\mathrm{Cl}$ 0.68-0.73) [13]. In the prospective study of O'Connor et al., the use of GerontoNet risk score incorrectly marked $38 \%$ of patients as low risk ADR group [19]. ADR prediction tools should be ideally tested on representative sample of a target population to evaluate clinical impact before widespread implementation.

Possible explanations why our results are different from those in the original GerontoNet ADR risk score study may be related to the interpretation of the variables within the score. In addition, calculation of the risk score in individuals has the potential to vary across different countries.

\section{Conclusion}

The results of our study suggest that ADRs in the elderly can be predicted with the use of GerontoNet Risk Score, although it improperly classified $21.9 \%$ of patients as high-risk group. The prevalence of a high number of prescriptions ( $>7$ drugs) in study population elevated GerontoNet risk score by 4 points may contribute to improper prediction rate.

\section{Author Contributions}

Sergey Zyryanov, who is the senior author, provided the intellectual input and approved the protocols to be followed in the study. Ali Al-Ragawi, who is the presenting author, is responsible for preparing the manuscript and analysing data. Olga Butranova and Elena Ushkalova both contributed in the critical revision of the manuscript. Anton Pereverzev contributed in conception design and data collection.

\section{Funding}

No sources of funding were used to assist in the preparation of this article. 


\section{Competing Interests}

The authors have declared that no competing interests exist.

\section{References}

1. https://www.prb.org/2018-world-population-data-sheet-with-focus-on-changing-agestructures/

2. http://ac.gov.ru/files/publication/a/8485.pdf.

3. Marengoni A, Winblad B, Karp A, Fratiglioni L. Prevalence of chronic diseases and multimorbidity among the elderly population in Sweden. Am J Public Health. 2008; 98: 11981200.

4. Melis R, Marengoni A, Angleman S, Fratiglioni L. Incidence and predictors of multimorbidity in the elderly: a population-based longitudinal study. PLoS One. 2014; 9: e103120.

5. Barnett K, Mercer SW, Norbury M, Watt G, Wyke S, Guthrie B. Epidemiology of multimorbidity and implications for health care, research, and medical education: A cross sectional study. Lancet. 2012; 380: 37-43.

6. Guthrie B, Makubate B. The rising tide of polypharmacy and potentially serious drug interactions 1995-2010: Repeated cross sectional analysis of prescribing in one region. Prim Health Care Dev. 2012; 13: 45 2E.2, doi: 10.1186/s12916-015-0322-7

7. Storms H, Marquet K, Aertgeerts B, Claes N. Prevalence of inappropriate medication use in residential long-term care facilities for the elderly: A systematic review. Eur J Gen Pract. 2017; 23: 69-77.

8. Dilles $T$, Vander Stichele R, Van Bortel L, Elseviers $M$. The development and test of an intervention to improve ADR screening in nursing homes. J Am Med Dir Assoc. 2013; 14: 379.e1-379.e6.

9. Kongkaew C, Noyce PR, Ashcroft DM. Hospital admissions associated with adverse drug reactions: a systematic review of prospective observational studies. Ann Pharmacother. 2008; 42: 1017-1025.

10. Alhawassi TM, Krass I, Bajorek BV, Pont LG. A systematic review of the prevalence and risk factors for adverse drug reactions in the elderly in the acute care setting. Clin Interv Aging. 2014; 9: 2079-2086.

11. Prybys K, Melville K, Hanna J, Gee A, Chyka P. Polypharmacy in the elderly: Clinical challenges in emergency practice: Part 1: Overview, etiology, and drug interactions. Emerg Med Rep. 2002; 23: 145-153.

12. Beijer HJ, de Blaey CJ. Hospitalisations caused by adverse drug reactions (ADR): A metaanalysis of observational studies. Pharm World Sci. 2002; 24: 46-54.

13. Onder G, Petrovic M, Tangiisuran B, Meinardi MC, Markito-Notenboom WP, Somers A, et al. Development andvalidation of a score to assess risk of adverse drug reactionsamong inhospital patients 65 years or older. Arch Intern Med. 2010; 170: 1142-1148.

14. Petrovic M, Tangiisuran B, Rajkumar C, van der Cammen T, Onder G. Predicting the risk of adverse drug reactions in older inpatients: External validation of the GerontoNet ADR risk score using the CRIME Cohort. Drugs Aging. 2017; 34: 135-142. 
15. Masnoon N, Shakib S, Kalisch-Ellett L, Caughey GE. What is polypharmacy? A systematic review of definitions. BMC Geriatr. 2017; 17: 230.

16. Haider SI, Ansari Z, Vaughan L, Matters H, Emerson E. Prevalence and factors associated with polypharmacy in Victorian adults with intellectual disability. Res Dev Disabil. 2014; 35: 30713080.

17. Best O, Gnjidic D, Hilmer SN, Naganathan V, McLachlan AJ. Investigating polypharmacy and drug burden index in hospitalised older people. Inter Med Jour. 2013; 43: 912-918.

18. Frazier SC. Health outcomes and polypharmacy in elderly individuals: an integrated literature review. J Gerontol Nurs. 2005; 31: 4-11.

19. Payne R, Avery A, Duerden M, Saunders C, Simpson C, Abel G. Prevalence of polypharmacy in a Scottish primary care population. Eur J Clin Pharmacol. 2014; 70: 575-581.

20. Poudel A, Hubbard RE, Nissen L, Mitchell C. Frailty: A key indicator to minimize inappropriate medication in older people. QJM. 2013; 106: 969-975.

21. O'Connor M, Gallagher P, Byrne S, O'Mahony D. Adverse drug reactions in older patients during hospitalisation: Are they predictable? Age Ageing. 2012; 41: 771-776.

22. Lavan AH, Gallagher P. Predicting risk of adverse drug reactions in older adults. Ther Adv Drug Saf. 2016; 7: 11-22.

23. Walckiers D, Van der Heyden J, Tafforeau J. Factors associated with excessive polypharmacy in older people. Arch Public Health. 2015; 73: 50.

24. Maher R, Hanlon J, Hajjar E. Clinical consequences of polypharmacy in elderly. Expert Opin Drug Saf. 2014; 13: 57-65.

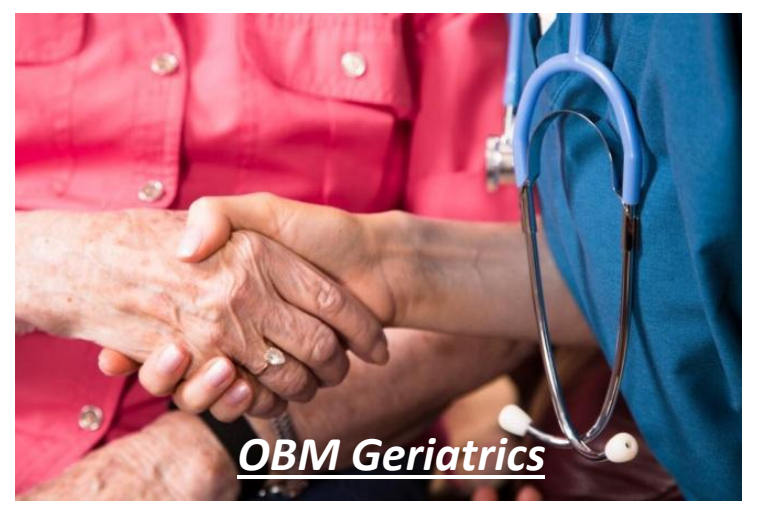

Enjoy OBM Geriatrics by:

1. Submitting a manuscript

2. Joining in volunteer reviewer bank

3. Joining Editorial Board

4. Guest editing a special issue

For more details, please visit: http://www.lidsen.com/journals/geriatrics 
Original Research

\title{
Exploring the Paradox of Care: Differences in Older Adult and Proxy Ratings of Health and Subjective Well-Being
}

Kristopher M. Struckmeyer ${ }^{1,}{ }^{*}$, Alex J. Bishop ${ }^{1,}{ }^{*}$, Brenda J. Smith $^{2}$, Brandt C. Gardner ${ }^{1}$

3. Department of Human Development \& Family Science, Oklahoma State University, 233 Human Sciences, Stillwater, Oklahoma, USA; E-Mails: struckm@okstate.edu; alex.bishop@okstate.edu; brandt.gardner@okstate.edu

4. Department of Nutritional Sciences, Oklahoma State University, 301 Human Sciences, Stillwater, Oklahoma, USA; E-Mail: bjsmith@okstate.edu

* Correspondences: Kristopher M. Struckmeyer, Alex J. Bishop; E-Mails: struckm@okstate.edu; alex.bishop@okstate.edu

Academic Editor: Lisa Hollis-Sawyer

Special Issue: Got Aging? Examining Later-life Development from a Positive Aging Perspective

OBM Geriatrics

2019, volume 3, issue 1

doi:10.21926/obm.geriatr.1901037
Received: August 27, 2018

Accepted: February 21, 2019

Published: February 27, 2019

\begin{abstract}
Background: The purpose of this study was to examine discrepancies in self-proxy agreement of biopsychosocial factors and the influence of objective well-being and psychosocial resources self and proxy appraisals of subjective well-being in older adults.

Methods: Seventy-two older adult-proxy dyads rated the biopsychosocial health of noncognitively impaired older adults.

Results: Proxies underestimated functional ability, psychosocial factors, and overrated negative health outcomes compared to older adults. Older adult ratings of place attachment $(\beta=.39, p<.01)$ and relationship quality $(\beta=.39, p<.01)$ were significant predictors of their subjective well-being. Proxies focused on both objective well-being and psychosocial resources to rate the older adult's well-being, especially in quality of life $\left(R^{2}=.87\right.$, adj. $R^{2}$ $=.82$ ).
\end{abstract}

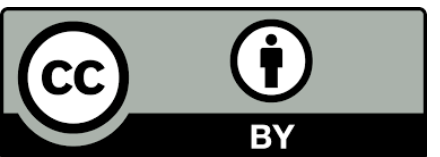

(C) 2019 by the author. This is an open access article distributed under the conditions of the Creative Commons by Attribution License, which permits unrestricted use, distribution, and reproduction in any medium or format, provided the original work is correctly cited. 
Conclusions: These findings indicate that discrepancies may exist between older adult-proxy ratings of health due to focusing on different components of well-being.

\section{Keywords}

Quality of life; self-appraisals; proxy appraisals; health agreement

\section{Introduction}

Americans are living longer with reduced risk of disease or limited functioning until much later in life [1]. Over the coming decades, this is expected to contribute to exponential growth in the number of persons living with functionally debilitating age-associated diseases such as Alzheimer's disease and other dementias, congestive heart failure, loss of vision and hearing, and osteoarthritis in very old age [2]. Reaching advanced older age and living with one or more diseases may negatively impact older adult (OA) well-being [3], possibly requiring assistance from a caregiver to meet daily tasks of living [4]. Instead of relying solely upon OA self-appraisals, a greater number of healthcare professionals seek information about the OA's well-being from a proxy, an individual (e.g., spouse, adult-child, friend) who provides information regarding the care recipient [5]. This is despite reported evidence that proxy appraisals can significantly differ from OA appraisals of well-being $[6,7]$. Nonetheless, investigators have made limited attempts to consider what the $\mathrm{OA}$ and proxy perceive as essential to quality-of-life. Therefore, the purpose of this investigation was to examine the influence of self-reported and proxy appraisals of OA objective health factors and psychosocial resources on OA well-being. This research has implications relative to how geriatric practitioners assess and consider positive aging in caregiving dyads.

\subsection{Conceptualizing Well-Being}

Well-being has generally been defined as the extent to which individual needs to produce positive feelings of satisfaction across various life domains (e.g., health functioning, social relationships, etc.)[8]. Well-being appraisal involves a high degree of inter-individual variability [3]. Individuals commonly filter perceptions and reactions through personal expectations, values, and history [9]. Nonetheless, Diener [10] posited two theoretical models of well-being to explain how persons appraise well-being. First, well-being is appraised top-down. In other words, it is hypothesized that persons are predisposed to view life events as positive or negative depending on life domains, thereby resulting in an optimistic opinion of some domains, or pessimistic evaluations of others [10]. Second, Diener [10] proposed that well-being is appraised from the bottom-up. This assumes that individuals simply summarize perceptions of well-being across life domains into a global or overall evaluation of their well-being [11]. Together, the top-down and bottom-up perspectives are composed of distinct physical, subjective, and psychological attributes that impact how persons positively appraise life [12]. 


\subsection{Self vs Proxy Appraisal of Well-Being}

Numerous studies have reported that self-appraisal is a better predictor of well-being than those obtained through a proxy $[13,14,15]$. Self-appraisals involve cognitive processes [16] that incorporate both past and ongoing health changes to create a global well-being appraisal [17]. However, because chronic conditions (e.g., dementia, cancer) can disrupt the lives of individuals trying to cope with the symptoms, proxies may be brought in to assess an individual's well-being in order to avoid methodological and analytical problems associated with missing data [18]. With one in ten older adults over the age of 65 diagnosed with Alzheimer's disease [19], self-appraisals are under further scrutiny for individuals diagnosed with dementia due to lack of personal insight associated with cognitive impairment [20]. Despite findings of reliable and consistent positive well-being ratings from individuals with mild to moderate dementia $[6,14]$, researchers still rely upon proxy ratings. It is assumed that the nominated proxy is intimately familiar with the OA [21]. However, several studies have cited discrepancies between self and proxy ratings of well-being. There is an evident lower discrepancy between self and proxy appraisals on physical functioning attributes of well-being, proxies tend to underestimate positive psychological aspects of aging (e.g., life satisfaction, happiness, etc.) [15, 20, 22]. Although self and proxy agreement on wellbeing has been previously examined in cognitively impaired older adults, there remains limited examination of self versus proxy agreement between non-cognitively impaired OAs and proxies. Well-being assessment by the $\mathrm{OA}$ and proxy requires all verbal and nonverbal communication cues [14], thus non-cognitively impaired older adults should be better able to communicate positive versus negative status of well-being to a proxy, increasing chances for higher agreement.

\subsection{Psychosocial Resources}

Given the challenge of assessing the well-being of old-old adults in various populations, Poon and colleagues [23] argue that psychosocial resources should alternatively be considered. Psychosocial resources are conceptualized as protective factors an individual can draw upon to indirectly or directly off-set the noxious impact of everyday stressors [24, 25]. Such resources may consist of inner strengths (e.g., spirituality, place attachment), as well as socio-environmental attachments (e.g., family, friends, neighbors) [26]. For instance, spirituality is an intra-personal dimension that produces meaning and transcendent experience beyond normative human consciousness [27] comes (e.g., morale, happiness, life satisfaction) [28]. Krause [29] noted that spirituality's deleterious effect on negative psychological outcomes, such as stress can be extended to the social network structure inherent within religious organizations. Membership within such congregations can influence emotional feelings of social belongingness and community membership. In turn, persons derive a sense of attachment to place or feeling of emotional comfort and security derived from belonging and connecting to the broader community. This perception of belonging to a community is especially important for aging individuals with limited mobility and health restrictions [30]. Oswald and colleagues argued that despite spending large portions of their time in their home, having a sense of belonging through friends and relatives in within the broader community contributes to a more positive aging experience.

The purpose of this study was to 1) determine the level of agreement between self and proxy appraisals of well-being, and 2) examine the association between perceived well-being and psychosocial resources relative to $\mathrm{OA}$ and proxy well-being assessment. Based on previous 
literature, we hypothesized that proxies would underestimate OA health status, perceived wellbeing, and psychosocial resources. We also predicted that OA's would associate more psychosocial resources with subjective well-being, while proxies would associate more health attributes with their OA's subjective well-being.

\section{Materials and Methods}

\subsection{Sample and Data Collection}

A convenience sample of $\mathrm{N}=72$ older adult-proxy dyads were recruited for this study. Such empirical comparison has largely focused on proxy ratings of cognitively impaired older adults, particularly those requiring long-term monitoring and assistance $[6,20]$. There is limited literature on dyadic comparisons of well-being appraisals between cognitively intact old adults and proxies. The OA were recruited first and upon completion of the study, were asked to nominate a proxy to complete a separate questionnaire. To be included in the study, the OA had to be 70 years of age and older and had to score 11 or higher on the Mini Mental State Exam Short- Form (MMSE-SF; [31]). Based on normative scoring ranges established for the MMSE-SF, those with a score below 11 were considered to be more likely to have cognitive health impairment [31]. The OA sample was recruited from two primary sources: First, community- dwelling older adults and individuals who resided in assisted living or nursing facilities in a south-central state were identified and recruited. Second, community dwelling older adults who resided in private homes were recruited from community locations such as senior activity centers, nutrition sites, and local churches. All participants were asked to read and sign a university approved informed consent. For those who may have had visual impairments which limited their ability to read, a trained-member of research team privately read the informed consent aloud one-to-one. Older adults completed a one-time questionnaire evaluating their current physical and psychosocial health. Upon completion of the questionnaire, the OA were compensated $\$ 25$ and were asked to nominate a proxy to complete a separate questionnaire. Initial recruitment efforts resulted in a sample of 165 older adults. All OAs nominated a proxy, but only 72 proxies responded (43.6\% response rate). All proxies included in this study were nominated first by the OA. No restrictions were placed on the OA regarding the proxy nomination. This was used in order to allow the OA the opportunity to nominate any individual whom they perceived to intimately know their well-being. Proxy ratings are often used to minimize burden on health-compromised older adults [18]. There were no inclusion criteria for the proxies to participate in the study. Upon nomination, proxies were mailed a packet containing information regarding the study and the questionnaire. Proxies completed a one-time questionnaire evaluating their appraisal of the older adult's physical and psychosocial health and returned the packet within seven days of receiving it. Upon completion of the survey, proxies were compensated \$15. All participants provided consent to participate in the study and this study was approved by authors' university Institutional Review Board. 


\subsection{Measures}

\subsubsection{Health}

Health was assessed using three variables: activities of daily living (ADL); psychological distress; and perceived health status.

Activities of daily living were assessed using the Activities of Daily Living questionnaire of the Older Americans' Resources and Services (OARS) Multidimensional Functional Assessment questionnaire [32]. The scale consisted of 13 items (e.g., can you use the telephone) rated on a three-point Likert scale ranging from "unable to do activity" (0) to "without help" (2), with higher scores indicating increased functional ability among the OA. The scale showed strong internal consistency for both the OA ( $\alpha=.79)$ and proxy sample $(\alpha=.91)$.

Psychological distress was assessed using the Brief Symptom Inventory [33]. The scale consisted of 18-items (e.g., nervousness or shakiness inside) on a five-point scale ranging from "not at all" (0) to "extremely" (4), with higher scores indicating increased psychological distress among the OA. The scale showed moderate internal consistency for the OA $(\alpha=.67)$ sample and strong internal consistency for the proxy sample $(\alpha=.94)$.

Perceived health status was assessed using one question that asked the individual to rate their current health. The question, "How would you rate your overall health at the present time," was rated using a four-point Likert scale ranging from "poor" (1) to "excellent" (4), with higher scores indicating better perceived health among the OA.

\subsubsection{Well-Being}

Six variables were used to measure well-being appraisal: Affect; gratitude; life satisfaction; purpose; happiness; and QoL.

Affect was measured using the Positive and Negative Affect Schedule (PANAS) to obtain scores on positive and negative psychological affect [34]. This scale consists of 20 items (e.g., interested [positive], distressed [negative]) on a five-point scale, ranging from "not at all" (1) to "extremely" (5). Scores range on both scales from 10-50. Composite scores were calculated by summing the positive items and the negative items. Higher scores represent higher levels of positive and negative affect. The positive affect subscale showed strong internal consistency for both the OA ( $\alpha$ $=.76)$ and proxy sample $(\alpha=.87)$. The negative affect subscale also showed strong internal consistency for both the OA ( $\alpha=.75)$ and proxy sample $(\alpha=.85)$.

Gratitude. was evaluated using the Gratitude Questionnaire-Six item short form [35]. Items (e.g., I have so much in life to be thankful for) were on a seven-point scale, ranging from "strongly disagree" (1) to "strongly agree" (7), with higher scores indicating increased gratitude among the $\mathrm{OA}$. The scale showed moderate internal consistency for the OA sample $(\alpha=.59)$ and strong internal consistency for the proxy sample $(\alpha=.82)$.

Life satisfaction. was assessed using the Satisfaction with Life Scale (SWLS) [36] to obtain scores on life satisfaction. The scale consisted of five items (e.g., in most ways, my life is close to ideal) using a seven-point scale ranging from "strongly disagree" (1) to "strongly agree" (7), with higher scores indicating increased life satisfaction among the OA. The scale showed strong internal consistency for both the OA $(\alpha=.73)$ and proxy sample $(\alpha=.88)$. 
Purpose in Life was evaluated using the Purpose in Life scale of the Ryff's Psychological Wellbeing scale (PWBI) [37]. This subscale consisted of seven items (e.g., I have a sense of direction and purpose in my life) rated on a six-point scale, ranging from "strongly disagree" (1) to "strongly agree" (7), with higher scores indicating increased purpose among the OA. The scale showed moderate internal consistency for the OA $(\alpha=.57)$, but a score could not be calculated for the proxy sample due to a negative average covariance among items.

Happiness was assessed using the Subjective Happiness Scale [38]. The scale consisted of four items (e.g., in general I consider myself happy) on a seven-point scale, ranging from one to seven, with higher scores indicating greater happiness. The scale showed strong internal consistency for both the OA $(\alpha=.73)$ and proxy sample $(\alpha=.82)$.

Quality of life was measured using the CASP-19 [39]. The scale consisted of 19 items (e.g., I can do the things I want to do) that are scored on a four-point Liker scale ranging from "never" (1) to "often" (4), with higher scores indicating increased QoL among the OA. The scale showed weak internal consistency for the OA $(\alpha=.28)$, but strong internal consistency for the proxy sample ( $\alpha$ $=.92)$.

\subsubsection{Psychosocial Resources}

Five variables were used to measure the individual's psychosocial resources: daily spiritual experiences; place attachment; relationship quality; social support; and loneliness.

Daily spiritual experiences were assessed using the Daily Spiritual Experiences Scale [40]. The scale consisted of 16 items (e.g., I feel God's presence) rated on a six-point Likert scale ranging from "never or almost never" (1) to "many times a day" (6), with higher scores indicating increased daily spiritual experiences among the OA. The scale showed strong internal consistency for both the OA $(\alpha=.87)$ and proxy sample $(\alpha=.94)$.

Place attachment was assessed using the Place Attachment Inventory [41]. The scale consisted of 12 items (e.g., this place is very special to me) on a five-point scale ranging from "strongly disagree" (1) to "strongly agree" (5), with higher scores indicating increased place attachment among the OA. The scale showed strong internal consistency for both the OA ( $\alpha=.89)$ and proxy sample $(\alpha=.94)$.

Relationship quality was assessed using a modified version of the Positive Affect Index [42]. The original scale consisted of 10-items (e.g., how well do you feel he/she understands you) on a sixpoint scale ranging from "not well" (1) to "extremely well" (6), with higher scores indicating increased relationship quality among the OA. Three questions were added to assess the OA's general affect towards their son or daughter. The scale showed strong internal consistency for both the OA $(\alpha=.82)$ and proxy sample $(\alpha=.92)$.

Social Support was assessed using the Social Provisions scale [43]. The measure was composed of 12 items (e.g., there are people I can depend on to help me if I really need it) on a four-point Likert scale ranging from "strongly disagree" (1) to "strongly agree" (4), with higher scores indicating increased social support among the OA. The scale showed moderate internal consistency for the OA sample $(\alpha=.67)$ and strong internal consistency for the proxy sample ( $\alpha$ $=.70)$.

Loneliness was assessed using the UCLA Loneliness scale [44], a 10-item (e.g., how often do you feel close to people) scale designed to obtain the participant's subjective feelings of loneliness and 
social isolation. The scale is on a four-point Likert scale ranging from "never" (1) to "always" (4), with higher scores indicating increased loneliness among the OA. The scale showed moderate internal consistency for the OA sample $(\alpha=.65)$ and strong internal consistency for the proxy sample $(\alpha=.72)$.

\subsection{Analytical Procedure}

Descriptive statistics were examined for the total sample. To examine self and proxy differences on health appraisals, we ran a paired sample t-test to analyze mean differences on all health variables. Third, we regressed the subjective health factors on the objective health factors and psychosocial resources for both the OA and proxy samples. Each linear regression was estimated by entering predictors with the ordering of demographics, objective health factors, and psychosocial resources. Given the small sample size and potential for bias from missing data, the variables were checked for potential issues. Missingness indicators were created and study variables were examined for potential relationships between variables and missingness. Several variables showed potential for being not being missing completely at random (MCAR) and so a multiple imputation model was used in Mplus 8 to create 20 datasets. The analysis was then rerun using these imputed datasets to help mitigate the potential bias of missing data. All analyses were examined using a significance level of .05 or less.

All participants provided consent to participate in the study and this study was approved by Oklahoma State University's Institutional Review Board (HE-1373) on September 13, 2014.

\section{Results}

\subsection{Descriptives}

The sample consisted of $\mathrm{N}=72$ dyads composed of an older adult and a nominated proxy. Mean age of older adult participants was 85.04 years $(S D=8.67)$, compared a mean age of 64.88 years $(S D=13.32)$ among nominated proxies. Frequencies and percentages for study variables are presented in Table 1 and Table 2.

Table 1 Frequencies and percentages of older adult and proxy samples.

\begin{tabular}{llllll}
\hline & \multicolumn{3}{l}{ Older Adult } & \multicolumn{2}{l}{ Proxy } \\
& $\mathbf{n}$ & Percentage & $\mathbf{n}$ & Percentage \\
\hline Gender & 17 & 23.6 & 18 & 25 \\
Male & 52 & 72.2 & 54 & 75 \\
Female & 3 & 4.2 & 0 & 0 \\
Missing & & & & \\
Race/Ethnicity & 0 & 0 & 0 & 0 \\
Alaskan Native & 0 & 0 & 3 & 4.2 \\
Asian/Asian American & 1 & 1.4 & 1 & 1.4 \\
Black/African American & 0 & 0 & 0 & 0 \\
Hispanic/Latino & 3 & 4.2 & 3 & 4.2 \\
Native American & 1 & 1.4 & 0 & 0 \\
Native Hawaiian and Pacific Islander & 63 & 87.5 & 65 & 90.3 \\
White/Caucasian & & & &
\end{tabular}




\begin{tabular}{|c|c|c|c|c|}
\hline Multi-Racial & 0 & 0 & 0 & 0 \\
\hline Missing & 4 & 5.6 & 0 & 0 \\
\hline \multicolumn{5}{|l|}{ Relationship Status } \\
\hline Never Married & 3 & 4.2 & 11 & 15.3 \\
\hline Married & 27 & 37.5 & 51 & 70.8 \\
\hline Divorced & 5 & 6.9 & 6 & 8.3 \\
\hline Widowed & 32 & 44.4 & 4 & 5.6 \\
\hline \multicolumn{5}{|l|}{ Education } \\
\hline Some high school or less & 6 & 8.3 & 0 & 0 \\
\hline Vocational, High School, GED & 18 & 25 & 9 & 12.5 \\
\hline Some college and Associates Degree & 18 & 25 & 8 & 11.1 \\
\hline College Degree & 6 & 8.3 & 19 & 26.4 \\
\hline $\begin{array}{l}\text { Some post graduate education, } \\
\text { Graduate education }\end{array}$ & 13 & 18.1 & 26 & 36.1 \\
\hline Ph.D./Doctoral Degree & 8 & 11.1 & 10 & 13.9 \\
\hline Missing & 3 & 4.2 & 0 & 0 \\
\hline
\end{tabular}

Table 2 Frequencies and percentages related to proxy specific descriptives.

\begin{tabular}{lll}
\hline & $\mathbf{n}$ & Percentage \\
\hline Relationship to Older Adult & & \\
Adult Child & 33 & 45.8 \\
Friend/Acquaintance & 10 & 13.9 \\
Nephew/Niece & 1 & 1.4 \\
Physician/Nurse & 8 & 11.1 \\
Sibling & 3 & 4.2 \\
Spouse & 14 & 19.4 \\
Other & 3 & 4.2
\end{tabular}

Distance to Older Adult

\begin{tabular}{lll} 
Live together & 17 & 23.6 \\
Less than 5 miles & 30 & 41.7 \\
5-50 miles & 12 & 16.7 \\
51-150 miles & 6 & 8.3 \\
151-250 miles & 2 & 2.8 \\
251-500 miles & 1 & 1.4 \\
More than 500 miles & 4 & 5.6 \\
Weekly Communication & & \\
None & 3 & 4.2 \\
1-2 times & 13 & 18.1 \\
3-4 times & 21 & 29.2 \\
5-8 times & 14 & 19.4 \\
9 or more & 21 & 29.2 \\
\hline
\end{tabular}




\subsection{Paired-Samples T-test}

Paired-samples t-tests were used to analyze the significant mean differences of the study variables between the two samples (See Table 3). On average, proxies tended to underrate their older adult's activities of daily living $(M=1.98)$, happiness $(M=2.77)$, gratitude $(M=2.13)$, life satisfaction $(M=2.95)$, place attachment $(M=4.59)$, and relationship quality $(M=2.81)$, and while overrating psychological distress $(M=-5.76)$ and loneliness $(M=-1.86)$.

Table 3 Paired sample t-test descriptives.

\begin{tabular}{|c|c|c|c|c|c|c|}
\hline & $\begin{array}{l}\text { Older Adult } \\
\text { Means }\end{array}$ & $\begin{array}{l}\text { Proxy } \\
\text { Means }\end{array}$ & $\begin{array}{l}\text { Mean } \\
\text { Difference }\end{array}$ & SD & $\mathbf{t}$ & $\mathbf{N}$ \\
\hline \multicolumn{7}{|l|}{ Underrated } \\
\hline ADL & 24.05 & 22.07 & $1.98 * * *$ & 3.68 & 4.21 & 61 \\
\hline Perceived Health & 2.88 & 2.70 & .18 & .94 & 1.57 & 67 \\
\hline Positive Affect & 34.66 & 33.13 & 1.53 & 8.97 & 1.24 & 53 \\
\hline Happiness & 22.73 & 19.95 & $2.77^{* *}$ & 5.25 & 3.66 & 48 \\
\hline Gratitude & 38.69 & 36.56 & $2.13^{*}$ & 6.61 & 2.23 & 48 \\
\hline Life Satisfaction & 28.18 & 25.23 & $2.95 * *$ & 7.91 & 3.01 & 65 \\
\hline $\begin{array}{l}\text { Daily Spiritual } \\
\text { Experiences }\end{array}$ & 62.37 & 59.65 & 2.73 & 13.94 & 1.40 & 51 \\
\hline Place Attachment & 44.37 & 39.78 & $4.59 * *$ & 12.21 & 2.98 & 63 \\
\hline Relationship Quality & 72.03 & 69.22 & $2.81 * *$ & 7.34 & 3.07 & 64 \\
\hline Social Support & 40.42 & 39.78 & .64 & 4.71 & 1.05 & 59 \\
\hline \multicolumn{7}{|l|}{ Overrated } \\
\hline $\begin{array}{l}\text { Psychological } \\
\text { Distress }\end{array}$ & 6.09 & 11.85 & $-5.76 * *$ & 12.72 & -3.07 & 46 \\
\hline Negative Affect & 15.67 & 15.83 & -.17 & 6.25 & -.20 & 54 \\
\hline Quality of life & 57.47 & 58.42 & -.94 & 10.27 & -.67 & 53 \\
\hline Loneliness & 19.28 & 21.14 & $-1.86 * *$ & 4.78 & -3.11 & 64 \\
\hline
\end{tabular}

Note. ${ }^{*} p<0.05 .{ }^{* *} p<0.01 .{ }^{* * *} p<0.001$

\subsection{Multivariate Findings}

Multiple linear regression analyses were conducted to assess the impact of self and proxy appraisals of objective health factors and psychosocial resources on subjective health factors of OA. Seven separate regression analyses were conducted for the OA sample and six separate regression analyses were conducted for the proxy sample to individually assess factors relevant to appraisals of well-being within each subsample. Linear order of entry for the variables was the same for all analyses, entered with the ordering of demographics (i.e., age, gender, and education), 
objective health variables (i.e., perceived health, psychological distress, and ADLs), and psychosocial resources (i.e., daily spiritual experiences, place attachment, relationship quality, loneliness, and social provision). The subjective health criterion variables included gratitude, life satisfaction, happiness, positive and negative affect, and quality of life. Purpose in life was a criterion variable for the OA sample, but was excluded from the proxy sample due to the scale's unreliability with the sample.

Table 4 presents the results of OA subjective health factors regressed on self-appraisals of objective health factors and psychosocial resources. In the OA sample model predicting positive affect, the predictors explained $35 \%$ of the variance. It was found that place attachment significantly predicted negative affect $(\beta=.24, p<.05)$. In the model predicting negative affect, the predictors explained $41 \%$ of the variance. It was found that age $(\beta=-.25, p<.05)$, psychological distress $(\beta=.53, p<.001)$, and daily spiritual experiences $(\beta=.33, p<.01)$ significantly predicted negative affect. The predictors explained $30 \%$ of the variance in happiness. Age $(\beta=-.27, p<.01)$ and relationship quality $(\beta=.37, p<.01)$ was found to significantly predict happiness. Predictors explained $22 \%$ of the variance in life satisfaction. Psychological distress ( $\beta$ $=.10, p<.001)$, place attachment $(\beta=.13, p<.01)$, and relationship quality $(\beta=-.07, p<.05)$ was found to significantly predict life satisfaction. The predicators explained $47 \%$ of the variance in quality of life. Age $(\beta=.08, p<.05)$ was found to significantly predict quality of life.

Table 5 presents the results of proxy appraisals of OA subjective health factors regressed on proxy appraisals of objective health factors and psychosocial resource. In the proxy sample model predicting positive affect, the predictors explained $58 \%$ of the variance. Activities of daily living ( $\beta$ $=.29, p<.05)$ and daily spiritual experiences $(\beta=.42, p<.001)$ were found to significantly predict positive affect. The predictors explained $53 \%$ of the variance in negative affect. Psychological distress $(\beta=.53, p<.001)$ was found to significantly predict negative affect. The model of happiness explained $60 \%$ of the variance. Gender $(\beta=.25, p<.05)$ and daily spiritual experiences $(\beta=.39, p<.01)$ were found to significantly predict happiness. The model of gratitude explained $68 \%$ of the variance. Daily spiritual experiences $(\beta=.42, p<.001)$, place attachment $(\beta=.31, p$ $<.01)$, and social support $(\beta=.30, p<.01)$ were found to significantly predict gratitude. In the model predicting life satisfaction, the predictors explained $76 \%$ of the variance. Age $(\beta=.15, p$ $<.05)$, daily spiritual experiences $(\beta=.36, p<.001)$, place attachment $(\beta=.37, p<.001)$, and loneliness $(\beta=-.41, p<.001)$ were found to significantly predict life satisfaction. In the model of QoL, the predictors explained $84 \%$ of the variance. Activities of daily living $(\beta=.33, p<.001)$, perceived health $(\beta=.23, p<.001)$, daily spiritual experiences $(\beta=.21, p<.01)$, place attachment $(\beta=.24, p<.001)$, and loneliness $(\beta=-.35, p<.001)$ were found to be significant predictors of QoL. 
Table 4 Predicting well-being on older care recipient sample $(N=72)$.

\begin{tabular}{|c|c|c|c|c|c|c|c|c|c|c|c|c|}
\hline \multirow[b]{2}{*}{ Predictors } & \multicolumn{2}{|c|}{ Positive Affect } & \multicolumn{2}{|c|}{ Negative Affect } & \multicolumn{2}{|c|}{ Happiness } & \multicolumn{2}{|c|}{ Gratitude } & \multicolumn{2}{|c|}{ Life Satisfaction } & \multicolumn{2}{|c|}{ Quality of Life } \\
\hline & $\beta$ & S.E. & $\beta$ & S.E. & $\beta$ & S.E. & $\beta$ & S.E. & $\beta$ & S.E. & $\beta$ & S.E. \\
\hline Age & -.07 & .13 & $-.25 *$ & .12 & $-.27^{*}$ & .13 & -.33 & .13 & -.03 & .15 & $.08^{*}$ & .11 \\
\hline Gender & .17 & .10 & .14 & .10 & .01 & .11 & -.04 & .11 & .10 & .12 & -.09 & .09 \\
\hline Education & .13 & .11 & .19 & .10 & .15 & .12 & -.04 & .11 & .08 & .12 & .00 & .10 \\
\hline Psychological Distress & -.02 & .12 & $.53 * * *$ & .11 & -.13 & .13 & .12 & .13 & $.10^{* * *}$ & .15 & -.36 & .10 \\
\hline Activities of Daily Living & .12 & .13 & .13 & .12 & -.13 & .14 & .08 & .14 & .04 & .15 & -.20 & .12 \\
\hline Perceived Health & .16 & .12 & .12 & .11 & .01 & .13 & .11 & .12 & .18 & .15 & .05 & .11 \\
\hline Daily Spiritual Experiences & .01 & .12 & $.33^{* *}$ & .11 & .04 & .13 & -.15 & .13 & .08 & .13 & -.25 & .10 \\
\hline Place Attachment & $.24^{*}$ & .12 & .08 & .12 & .12 & .13 & .11 & .13 & $.13^{* *}$ & .15 & .35 & .11 \\
\hline Relationship Quality & .11 & .12 & .05 & .11 & $.37 * *$ & .12 & .22 & .17 & $-.07 *$ & .13 & .24 & .10 \\
\hline Loneliness & -.12 & .13 & .14 & .13 & -.08 & .14 & .10 & .14 & -.26 & .14 & -.13 & .12 \\
\hline Social Support & .19 & .13 & -.02 & .13 & -.09 & .15 & .22 & .15 & .14 & .14 & .11 & .11 \\
\hline$R^{2}$ & .35 & & .41 & & .30 & & .28 & & .22 & & .47 & \\
\hline
\end{tabular}


Table 5 Predicting well-being based on proxy sample $(N=72)$.

\begin{tabular}{|c|c|c|c|c|c|c|c|c|c|c|c|c|}
\hline \multirow[b]{2}{*}{ Predictors } & \multicolumn{2}{|c|}{ Positive Affect } & \multicolumn{2}{|c|}{ Negative Affect } & \multicolumn{2}{|c|}{ Happiness } & \multicolumn{2}{|l|}{ Gratitude } & \multicolumn{2}{|c|}{ Life Satisfaction } & \multicolumn{2}{|c|}{ Quality of Life } \\
\hline & $\beta$ & S.E. & $\beta$ & S.E. & $\beta$ & S.E. & $\beta$ & S.E. & $\beta$ & S.E. & $\beta$ & S.E. \\
\hline Age & .11 & .09 & -.17 & .10 & .20 & .11 & -.06 & .08 & $.15^{*}$ & .07 & .08 & .06 \\
\hline Gender & .13 & .10 & -.09 & .10 & $.25^{*}$ & .11 & .06 & .08 & .01 & .07 & .05 & .06 \\
\hline Education & .04 & .10 & -.15 & .11 & -.00 & .09 & .07 & .09 & .13 & .07 & .10 & .06 \\
\hline Psychological Distress & -.17 & .14 & $.53^{* * *}$ & .13 & .12 & .16 & .17 & .16 & -.02 & .09 & .02 & .08 \\
\hline Activities of Daily Living & $.29 *$ & .14 & .25 & .13 & .20 & .12 & .09 & .13 & .08 & .09 & $.33 * * *$ & .08 \\
\hline Perceived Health & .17 & .10 & .05 & .11 & .04 & .10 & .12 & .09 & .15 & .08 & $.23 * * *$ & .06 \\
\hline Daily Spiritual Experiences & $.42 * * *$ & .12 & -.23 & .13 & $.39 * *$ & .13 & $.42 * * *$ & .10 & $.36^{* * *}$ & .09 & $.21^{* *}$ & .08 \\
\hline Place Attachment & .18 & .11 & -.19 & .11 & .11 & .10 & $.31 * *$ & .09 & $.37^{* * *}$ & .07 & $.24 * * *$ & .06 \\
\hline Relationship Quality & -.10 & .12 & .08 & .12 & .19 & .14 & .17 & .10 & -.14 & .09 & .04 & .08 \\
\hline Loneliness & -.18 & .13 & -.04 & .13 & -.21 & .13 & .02 & .20 & $-.41 * * *$ & .09 & $-.35 * * *$ & .08 \\
\hline Social Support & -.01 & .14 & -.23 & .13 & .18 & .16 & $.30 * *$ & .11 & .13 & .09 & .14 & .07 \\
\hline$R^{2}$ & .58 & & .53 & & .60 & & .68 & & .76 & & .84 & \\
\hline
\end{tabular}

Note. ${ }^{*} \mathrm{p}<0.05 .{ }^{* *} \mathrm{p}<0.01 .{ }^{* * *} \mathrm{p}<0.001$. 


\section{Discussion}

The aim of this study was to investigate the level of agreement between self and proxy appraisals of well-being. Our results confirmed that discrepancies persist between self and proxy appraisals. In particular, the results provide evidence regarding how OAs appraise their own wellbeing, as well as how proxies may perceive the $\mathrm{OA}^{\prime}$ 's well-being. Milne and colleagues [15] note that proxies have a tendency to underestimate psychosocial components of well-being due to the abstract nature and decreased visibility of the components. Consistent with previous findings [22, 45], proxies in this study underestimated their OA's functional ability. Although there was significant difference between self and proxy appraisals of functional ability, much of the discrepancy in self versus proxy agreement in this study centered around psychosocial components of well-being.

Our findings are consistent with previous work suggesting that OAs tend to focus on subjective attributes of well-being [6]. Carstensen, Isaacowitz, and Charles [46] argue that this is attributed to older adults use of emotion regulation to optimize their well-being in the face of shrinking time horizons. The Strengths and Vulnerability Integration (SAVI) model [47] further proposes that agerelated changes in perspective may be attributed to previous life experiences and an individual's perception of time left to live. In times of stress, individuals may employ emotion regulation skills through this changed perspective to minimize the experience of negative affect. According to Urry and Gross [48], the strategies individuals use to regulate emotion are based upon available resources, both internal and external. Data from this study suggests that relationship quality and place attachment are strongly associated with well-being. In late life, older adults place greater emphasis on building more positive social and community relationships within an intimate circle of friends [49]. Acquisition of material possessions tends to be secondary [49]. It is believed that the evolution of advanced human social systems has created a need to belong [50]. This is supported by relationship quality being a significant predictor of happiness. Socially intimate ties are among the most important attributes of positive well-being in later life [51, 52]. Social relationships can also provide the older individual with a sense of optimism about the future and opportunities to participate in their environment [53]. An older adult's relationship quality can affect emotional happiness and contribute to engagement within the community and overall well-being.

The theory of environmental press highlights the environmental challenges faced by older adults [54]. As communities change over time, the environment may not always be aging friendly and some older adults may lack the resources to adapt successfully to the negative environmental conditions [55]; increasing the OA's vulnerability for feeling ostracized [50] and decreased wellbeing in later life. It can be speculated that psychological distress (e.g., depression, anxiety) can limit an older adult's motivation to seek emotionally gratifying activities. This limited environmental engagement can have a detrimental impact on life satisfaction and positive aging.

Results from this study further suggest that proxies focus more objective attribute of well-being factors and psychosocial resources when appraising old-old adults well-being. However, this may be related to the proxies' preconceived perceptions of late life. According to Labelling theory, individuals integrate age stereotypes into their self-evaluations, thus influencing appraisals of SWB [56]. Cho, Martin, Margrett, MacDonald, and Poon [57] argue that an individual's attitudes and beliefs influence perceptions of health, so it can be speculated that proxies integrate age 
stereotypes into their appraisals of their OA's well-being [58]. Other investigators have also reported that proxy appraisals of OA well-being can be influenced by the proxy's own mental health [59], pain [22], and depression [60]. In this study, this was supported by proxy overestimate of OA loneliness. Loneliness was a major predictor for proxies' assessment of OA life satisfaction and quality of life, but was not significant for OA self-appraisals of the same variables. It is plausible that proxies may rate OA loneliness based on the ageist assumption that older adults are lonelier due to normative age-associated losses in biological, psychological, and social functioning. This finding may also be related to proxy experience of isolation depending on whether the proxy may provide care to the OA [61]. Developmentally, the experience of loss is normative [62]. The $O A$ 's lack of focus on loneliness may be attributed to socioemotional selectivity theory in which older adults normatively adapt to loss by minimizing negative emotional relationships [46]. Older adults may obtain the companionship they need from their proxies, viewing the relationship as fulfilling and as a source of strength. However, the proxy may engage in social comparison, assuming that the older adult should have more relationships because the proxy would want a large quantity of relationships in later life [63]. Thus, while the older adult may view the proxy as his or her one true source of social intimacy, the proxy may view the small social networks as a negative or a sign of vulnerability. Further research is needed to examine this association.

An important contribution of the present study is that the discrepancies in self versus proxy agreement may be attributed to $\mathrm{OA}$ and proxy examination of well-being through different adaptive behaviors. Due to shrinking time horizons, OAs may be focused on "in the moment" wellbeing to ensure that they optimize their SWB and minimize their negative affect. Older adults may not focus on objective aspects such as functional limitations due to adaptation. Pavot and Diener [64] noted that positive or negative changes in life events can influence an individual's SWB, but only temporarily. Thus, OAs may adapt to negative health changes, returning to their baseline SWB overtime; indicative of a top-down approach to well-being. OAs may use these emotional regulation strategies to maintain a positive predisposition in which to examine various life domains. Perhaps, it can be argued that the psychosocial resources play a key role in how OAs adapt to negative changes and maintain a positive sense of aging. Further research will be needed to investigate this association.

Interestingly, proxies focused intently on the OAs quality-of-life. There has been to no research which has examined why proxies focus exclusively on quality-of-life, but there are two possibilities. First, the focus may be attributed to the health. First, proxies may strive to maximize OA health provided that this plays an instrumental part in quality-of-life [15]. Second, Torke, Schwartz, Holtz, Montz, and Sachs [65] speculate that proxies' current preferences for their own future aging may influence a focus of OA biopsychosocial functioning. In other words, proxies may perceive that they would want someone to care pay attention to their biopsychosocial needs in old age and therefore so should the OA.

This study has provided some preliminary evidence concerning the influence of objective and psychosocial factors on SWB. It is, however, important to acknowledge the limitations of this study. First, methodological limitations included a small sample size and a cross-sectional design. Several of the measures had low reliability scores, indicating that the scales were not capturing the concept they were originally intended to measure. Although most of the measures were in the acceptable range from exploratory studies (greater or equal to .60) [66], quality of life was very low for the OA sample. One issue might be that psychometrics for these scales were calculated 
using younger populations and not elders. For example, while the CASP-19 was initially developed using a population aged 65-75 years [67], the mean age of the OA sample was outside of this range, thus the scale might not capture the characteristics of the oldest-old. Second, the sample was selected from a semi-rural state, so it is unlikely that it will be representative of the caregiving-care recipient dyad population as a whole. In addition, studies should follow dyads over time to examine how these discrepancies change over time, particularly examining cohort differences. Some of the findings, such as proxies' focus on quality of life, may be attributed to cohort effects. With these limitations, it is important to caution interpretation of these findings as the results are modestly statistically significant. Future research might also look at the context in which care is being provided. Some individuals in this sample resided with their proxies, while others resided in a long-term care facility.

\section{Conclusions}

Findings from this study indicate that regardless of cognitive impairment status, discrepancies exist between self and proxy appraisals of biopsychosocial health factors. This implies that proxies may not fully understand their older adults' well-being, underscoring the need to better understand what influences older adult and proxy well-being appraisal. Investigators have noted greater self-proxy agreement for physical health factors with low agreement for psychosocial components of well-being [15]. Future quantitative studies with larger samples are needed to examine self-proxy agreements of biopsychosocial health factors in OAs.

This study's findings lend to the importance of obtaining ratings from both OAs and proxies to better develop a more picture of the OA's well-being. Older adults appear to be focused on experiential well-being, while proxies are focused on their overall well-being. Because proxies are not focused on the same factors as their OAs, this may cause some friction. Educating proxies about this narrowed focus may alleviate some of the tension between proxy and OA.

\section{Acknowledgements}

The authors would like to thank Dr. Isaac Washburn for his aid with the missing data analyses. We would also like to thank Brooke Harris, a graduate student at Northeastern University, and Jamye Danielsen for their assistance with data collection.

\section{Author Contributions}

Bishop, Smith, Gardner: concept, design, preparation of paperwork. Struckmeyer, Bishop: analysis, interpretation. Struckmeyer: preparation of paper. Bishop: continual edits throughout process.

\section{Funding}

This study was funded by the Oklahoma Center for the Advancement of Science (OCAST) Health Research Program, OCAST Project \#HR13-174. 


\section{Competing Interests}

The authors have declared that no competing interests exist.

\section{References}

79. Talley RC, Crews JE. Framing the public health of caregiving. Am J Public Health. 2007; 97: 224-228.

80. Zolnikov TR. Proposing a re-adapted successful aging model addressing chronic diseases in low- and middle-income countries. Qual Life Res. 2015; 24: 2945-2949.

81. Netuveli G, Blane D. Quality of life in older ages. Br Med Bull. 2008; 85: 113-126.

82. Nguyen V. Long-term support and services. 2017 Retrieved from http://www.aarp.org/content/dam/aarp/ppi/2017-01/Fact\%20Sheet\%20Long-

Term\%20Support\%20and\%20Services.pdf

83. Lyons KS, Zarit SH, Sayer AG, Whitlatch CJ. Caregiving as a dyadic process: Perspectives from caregiver and receiver. J Gerontol B Psychol Sci Soc Sci. 2002; 57B: P195-P204.

84. Arlt $S$, Hornung J, Eichenlaub $M$, Jahn $H$, Bullinger, $M$, Petersen $C$. The patient with dementia, the caregiver and the doctor: Cognition, depression and quality of life from three perspectives. Int J Geriatr Psychiatry. 2008; 23: 604-610.

85. Trigg R, Watts $S$, Jones $R$, Tod A, Elliman R. Self-reported quality of life ratings of people with dementia: The role of attitudes to aging. Int Psychogeriatr. 2012; 24: 1085-1093.

86. Costanza R, Fisher B, Ali S, Beer C, Bond L, Boumans R, et al. Quality of life: An approach integrating opportunities, human needs, and subjective well-being. Ecol Econ. 2007; 61: 267276.

87. Albuquerque I, Lima M, Matos M, Figueiredo C. Work matters: Work personal projects and the idiosyncratic linkages between traits, eudaimonic, and hedonic well-being. Soc Indic Res. 2014; 115: 885-906.

88. Diener E. Subjective well-being. Psychol Bull. 1984; 95: 542-575.

89. Feist GJ, Bodner TE, Jacobs JF, Miles M, Tan V. Integrating top-down and bottom-up structural models of subjective well-being: A longitudinal investigation. J Pers Soc Psychol. 1995; 68: 138-150.

90. Ardelt M. Disentangling the relations between wisdom and different types of well-being in old age: Findings from a short-term longitudinal study. J Happiness Stud. 2016; 17: 1963-1984.

91. Angner E, Ray MN, Saag KG, Allison JJ. Health and happiness among older adults: A community-based study. J Health Psychol. 2009; 14: 503-512.

92. Lukas A, Niederecker T, Günther I, Mayer B, Nikolaus T. Self- and proxy report for the assessment of pain in patients with and without cognitive impairment. Z Gerontol Geriatr. 2013; 46: 214-221.

93. Milne DJ, Mulder LL, Beelen HCM, Schofield P, Kempen GIJM, Aranda S. Patients' self-report and family caregivers' perception of quality of life in patients with advanced cancer: How do they compare? Eur J Cancer Care. 2006; 15: 125-132.

94. Jylhä M. What is self-rated health and why does it predict mortality? Towards a unified conceptual model. Soc Sci Med. 2009; 69: 307-316.

95. Bailis DS, Segall A, Chipperfield JG. Two views of self-rated general health status. Soc Sci Med. 2003; 56: 203-217. 
96. Moinpour CM, Lyons B, Schmidt SP, Chansky K, Patchell RA. Substituting proxy ratings for patient ratings in cancer clinical trials: An analysis based on a southwest oncology group trial in patients with brain metastases. Qual Life Res. 2000; 9: 219-231.

97. Alzheimer's Association. 2017 Alzheimer's disease facts and figures. 2017. Retrieved from http://www.alz.org/documents_custom/2017-facts-and-figures.pdf

98. Trigg $R$, Watts $S$, Jones $R$, Tod $A$. Predictors of quality of life ratings from persons with dementia: The role of insight. Int J Geriatr Psychiatry. 2011; 26: 83-91.

99. Kim EJ, Song DH, Kim SJ, Park JY, Lee E, Seok JH, et al. Proxy and patients ratings on quality of life in patients with schizophrenia and bipolar disorder in Korea. Qual Life Res. 2010; 19: 521529.

100. Orgeta V, Orrell M, Edwards RT, Hounsome B, Woods B, REMCARE Team. Self- and carerrated pain in people with dementia: Influences of pain in carers. J Pain Symptom Manage. 2015; 49: 1042-1049.

101. Poon LW, Martin P, Bishop A, Cho J, da Rosa G, Deshpande N, et al. Understanding centenarians' psychosocial dynamics and their contributions to health and quality of life. Curr Gerontol Geriatr Res. 2010; 1-13.

102. Jeon HS, Dunkle RE. Stress and depression among the oldest-old: A longitudinal analysis. Res Aging. 2009; 31: 661-687.

103. Luo Y, Waite $\amalg$. Mistreatment and psychological well-being among older adults: Exploring the role of psychosocial resources and deficits. J Gerontol B Psychol Sci Soc Sci. 2011; 66B: 217229.

104. Henry CS, Hubbard R, Struckmeyer K, Spencer T. Family resilience and caregiving. New York: Springer; 2018. (p. 1-26).

105. Yoon DP. Factors affecting subjective well-being for rural elderly individuals. J Relig Spirit Soc Work. 2006; 25: 59-75.

106. Ellison CG, Fan D. Daily spiritual experiences and psychological well-being among US adults. Soc Indic Res. 2008; 88: 247-271.

107. Krause N. Exploring the stress-buffering effects of church-based and secular social support on self-rated health in late life. J Gerontol B Psychol Sci Soc Sci. 2006; 61B: S35-S43.

108. Oswald F, Jopp D, Rott C, Wahl HW. Is aging in place a resource for or a risk to life satisfaction? Gerontologist. 2010; 51: 238-250.

109. Folstein MF, Folstein SE, White T, Messer MA. MMSE-2: Mini-mental state examination (2 ${ }^{\text {nd }}$ Edition) users manual. PAR, Inc; 2010.

110. Fillenbaum GG, Smyer MA. The development, validity, and reliability of the OARS Multidimensional Functional Assessment questionnaire. J Gerontol. 1981; 36: 428-434.

111. Derogatis LR, Melisaratos N. The brief symptom inventory: An introductory report. Psychol Med. 1983; 13: 595-605.

112. Watson D, Clark LA, Tellegen A. Development and validation of brief measures of positive and negative affect: the PANAS scales. J Pers Soc Psychol. 1988; 54: 1063-1070.

113. McCullough ME, Emmons RA, Tsang J. The grateful disposition: A conceptual and empirical topography. J Pers Soc Psychol. 2002; 82: 112-127.

114. Diener E, Emmons RA, Larsen RJ, Griffin S. The satisfaction with life scale. J Pers Assess. 1985; 49: 71-75. 
115. Ryff C. Happiness is everything, or is it? Explorations on the meaning of psychological wellbeing. J Pers Soc Psychol. 1989; 57: 1069-1081.

116. Lyubomirsky S, Lepper H. A measure of subjective happiness: Preliminary reliability and construct validation. Soc Indic Res. 1999; 46: 137-155.

117. Hyde $M$, Wiggins RD, Higgs $P$, Blane DB. A measure of quality of life in early old age: The theory, development and properties of a needs satisfaction model (CASP-19). Aging Ment Health. 2003; 7: 186-194.

118. Underwood LG, Teresi JA. The Daily Spiritual Experience scale: Development, theoretical description, reliability, exploratory factor analysis, and preliminary construct validity using health-related data. Ann Behav Med. 2002; 24: 22-33.

119. Williams DR, Vaske JJ. The measurement of place attachment: Validity and generalizability of a psychometric approach. Forest Sci. 2003; 49: 830-840.

120. Bengtson VL, Schrader SS. Research Instruments in Social Gerontology. Minneapolis: University of Minnesota Press. 1982. (p. 115-185)

121. Cutrona CE, Russell D. Advances in personal relationships. Greenwich: JAI Press. 1987. (p. 3767),

122. Russell D, Peplau LA, Ferguson ML. Developing a measure of loneliness. J Pers Assess. 1978; 42: 290-294.

123. Punjabi NM. Sleep and quality of life in clinical medicine. Totowa: Humana Press. 2008. (p. 1117).

124. Carstensen LL, Isaacowitz DM, Charles ST. Taking time seriously: A theory of socioemotional selectivity. Am Psychol. 1999; 54: 165-181.

125. Charles ST. Strength and vulnerability integration: A model of emotional well-being across adulthood. Psychol Bull. 2010; 136: 1068-1091.

126. Urry HL, Gross JJ. Emotional regulation in older age. Curr Dir Psychol Sci. 2010; 19: 352-357.

127. Kyra-Lighthall N, Mather M. Handbook of Theories of Aging. New York: Springer. 2009. (p. 323-344).

128. Scannell L, Gifford R. Place attachment enhances psychological need satisfaction. Environ Behav. 2017; 49: 359-389.

129. Gustavson K, Roysamb E, Borren I, Torvik FA, Karevold E. Life satisfaction in close relationships: Findings from a longitudinal study. J Happiness Stud. 2016; 17: 1293-1311.

130. Adams RG, Blieszner R. Aging well with friends and family. Am Behav Sci. 1995; 39: 209-224.

131. Huxhold O, Miche M, Schüz B. Benefits of having friends in older ages: Differential effects of informal social activities on well-being in middle-aged and older adults. J Gerontol B Psychol Sci Soc Sci. 2013; 69: 366-375.

132. Lawton MP. Environment and other determinants of well-being in older people. Gerontologist. 1983; 23: 349-357.

133. Byrnes M, Lichtenberg PA, Lysack C. Environmental press, aging in place, and residential satisfaction of urban older adults. Socio Pract J Clin Appl Sociol. 2006; 23: 50-76.

134. Kotter-Grühn D, Hess TM. The impact of age stereotypes on self-perceptions of aging across the adult lifespan. J Gerontol B Psychol Sci Soc Sci. 2012; 67: 563-571.

135. Cho J, Martin P, Margrett J, MacDonald M, Poon LW. The relationship between physical health and psychological well-being among oldest-old adults. J Aging Res. 2011; 1-8. 
136. Umphrey D, Robinson T. Negative stereotypes underlying other-person perceptions of the elderly. Educ Gerontol. 2007; 33: 309-326.

137. Hoe J, Katona C, Orrell M, Livingston G. Quality of life in dementia: Care recipient and caregiver perceptions of quality of life in dementia: the LASER-AD study. Int J Geriatr Psychiatry. 2007; 22: 1031-1036.

138. Scocco P, Fantoni G, Caon F. Role of depressive and cognitive status in self-reported evaluation of quality of life in older people: Comparing proxy and physician perspectives. Age Ageing. 2006; 35: 166-171.

139. Bastawrous M. Caregiver burden - a critical discussion. Int J Nurs Stud. 2013; 50: 431-441.

140. Heckhausen J, Wrosch C, Schulz R. A motivational theory of life-span development. Psychol Rev. 2010; 117: 32.

141. Krueger J. Handbook of social comparison. New York: Kluwer Academic/Plenum Publishers; 2000. (p. 323-351).

142. Pavot W, Diener E. The Satisfaction with Life Scale and the emerging construct of life satisfaction. J Posit Psychol. 2008; 3: 137-152.

143. Torke AM, Schwartz PH, Holtz LR, Montz K, Sachs GA. Caregiver perspectives on cancer screening for persons with dementia: "Why put them through it?". J Am Geriatr Soc. 2013; 61: 1309-1314.

144. Nunnally JC, Bernstein IH. Psychometric theory. New York: McGraw-Hill; 1994.

145. Sim J, Bartlam B, Bernard M. The CASP-19 as a measure of quality of life in old age: Evaluation of its use in a retirement community. Qual Life Res. 2011; 20: 997-1004.

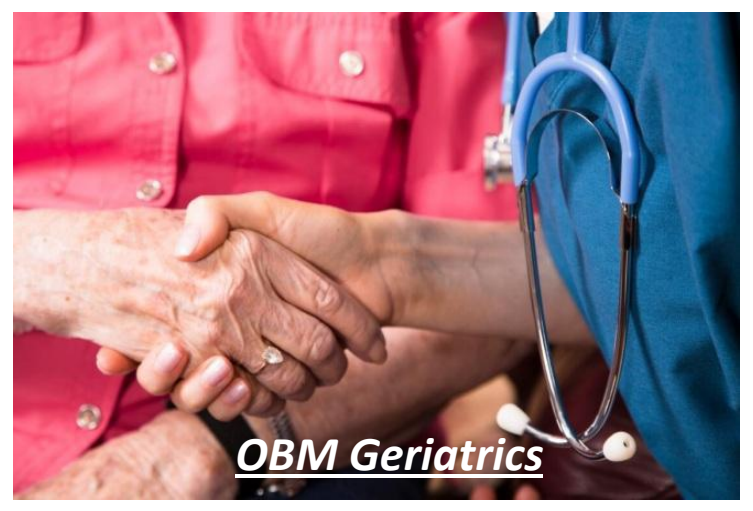

Enjoy $O B M$ Geriatrics by:

1. Submitting a manuscript

2. Joining in volunteer reviewer bank

3. Joining Editorial Board

4. Guest editing a special issue

For more details, please visit:

http://www.lidsen.com/journals/geriatrics 
Technical Note

\section{Improving the HealthCare of People with Dementia beyond the Diagnosis: The "Carlo Poma Dementia Care Pathway" Study Protocol}

Vincenza Frisardi ${ }^{1,}{ }^{*}$, Sara Faroni ${ }^{2}$, Angela Bellani ${ }^{2}$, Felice Biagi ${ }^{1}$, Emanuela Galante ${ }^{1}$, Alida Balzanelli ${ }^{1}$, Erika Talassi ${ }^{1}$, Lorella Frittoli $^{1}$, Giancarla Capiluppi ${ }^{2}$, Claudia D'angelis ${ }^{1}$, Antonella Taragnani ${ }^{2}$, Donatella Terzi ${ }^{2}$, Elena Podavini ${ }^{2}$, Graziana Gazzoni ${ }^{3}$, Graziana Simoncelli ${ }^{3}$, Carmine Matarazzo ${ }^{4}$, Mirko Avesani ${ }^{1}$, Antonio Ventura ${ }^{5}$, Consuelo Basili ${ }^{6}$, Maurizio Galavotti ${ }^{6}$, Carlo Maria Stucchi ${ }^{1}$, Maria Cristina Cilia ${ }^{1}$, Alfonso Ciccone ${ }^{1}$

1. Neurological Department, Asst Mantova, Carlo Poma Hospital, Mantua, Italy; E-Mails: vfrisardi@yahoo.com; felice.biagi@asst-mantova.it; emanuela.galante@asst-mantova.it; alida.balzanelli@asst-mantova.it; erika.talassi@asst-mantova.it; lorella.frittoli@asstmantova.it; claudia.dangelis@asst-mantova.it; mirko.avesani@asst-mantova.it; carlomaria.stucchi@asst-mantova.it; mariacristina.cilia@gmail.com; alfonso.ciccone@asstmantova.it

2. Frailty Department, Multiservice center, Asst Mantova Carlo Poma Hospital, Mantua, Italy; EMails: sara.faroni@asst-mantova.it; angela.bellani@asst-mantova.it; giancarla.capiluppi@asstmantova.it; antonella.taragnani@asst-mantova.it; donatella.terzi@asst-mantova.it; elena.podavini@asst-mantova.it

3. Frailty Department, Social Workers Office-Asst Mantova Carlo Poma Hospital, Mantua, Italy; EMails: graziana.gazzoni@asst-mantova.it; graziana.simoncelli@asst-mantova.it

4. Rehabilitation Department Asst Mantova, Bozzolo, Italy; E-Mail: carmine.matarazzo@asstmantova.it

5. Statistical Department Asst Mantova, Carlo Poma Hospital, Mantua, Italy; E-Mail: antonio.ventura@asst-mantova.it

6. Strategical Direction Department, Asst Mantova Carlo Poma Hospital, Mantua, Italy; E-Mails: consuelo.basili@asst-mantova.it; maurizio.galavotti@asst-mantova.it

* Correspondence: Vincenza Frisardi; E-Mail: vincenza.frisardi@asst-mantova.it

Academic Editor: Michael Fossel

OBM Geriatrics

2019 , volume 3 , issue 1
Received: October 31, 2018

Accepted: February 13, 2019

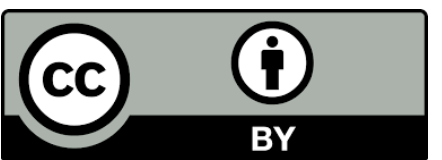

(C) 2019 by the author. This is an open access article distributed under the conditions of the Creative Commons by Attribution License, which permits unrestricted use, distribution, and reproduction in any medium or format, provided the original work is correctly cited. 


\begin{abstract}
The WHO global action plan on the public health response to dementia 2017-2025 stressed the need to have a comprehensive approach with deep interconnections and cross-cutting elements through several action areas. As the elderly population grows worldwide, the number of patients with dementia increases rapidly because age is an important risk factor for developing late-onset dementia. Currently, dementia syndrome represents a true emergency. Once a diagnosis of dementia was made, informal caregivers, patients and their household, they swing between desires, fear, concerns about the present and the future. In fact, in several clinical context there is not a possibility to have specific services able to address the critical issues, which could happen along the disease's course. Despite extensive research in the field of dementia, there still exist a deficiency in the quality of dementia care. There is a paucity of robust results concerning the care experiences of patients with dementia. It is mandatory to understand these experiences if we want to address care inequalities and create impactful dementia policies to improve services for supporting individuals and family caring, and promoting a good quality of life for all people affected by this devastating disease. Aim of this article is to describe the development of an operational protocol to improve the healthcare of people with cognitive impairment and their family. Unfortunately, it is not always easy to assure the whole services that a patient with dementia (PwD) needs. Frequently, this is due to a lack of communication between public hospitals and local authorities. We believe that it is possible to improve the quality of life of PWD and to optimize the public health expenditure through the creation of a specific care network. The services integration means to guarantee the continuity of care and the appropriateness of access to health care, avoiding inappropriate use. This could result in both reducing the healthcare costs and saving resources.
\end{abstract}

\title{
Keywords
}

Dementia care pathway; acute setting; organizational model

\section{Introduction}

Dementia is an umbrella term for several progressive neurodegenerative diseases. Sometimes, the first clinical manifestation is the occurrence of behavioral and psychological or motor disorders; thus, dementia is often underdiagnosed and mistreated for a long period of time [1]. Alzheimer's disease $(A D)$ is the most common form of dementia characterized by memory loss and impairment in cognitive and behavior abilities. It interferes significantly with the functional independence and social relationship [2] of people with dementia (PwD). The prevalence of Alzheimer's disease in Europe was estimated at 5.05\% (men 3.31\%; women, $7.13 \%$ ) while the incidence was 11.08 per 1000 person-years (7.02 per 1000 person-years in men and 13.25 in female per 1000 person-years in women) [3]. This phenomenon is expected to increase worldwide [4]. Recent update about age specific data from East Asia and Africa predicted an increase of $15 \%$ and 1\%, respectively, by 2030 
and 2050 on the overall world estimates, suggesting a very global explosion of this disease [2]. The World Health Organization (WHO) global action plan on the public health response to dementia 2017-2025 stressed the need to have a comprehensive and multisector approach with deep interconnections and cross-cutting elements through several action areas, with emphasis on promotion, prevention, treatment, rehabilitation and care [5]. This plan against dementia envisions "a world in which people can live well with or without dementia, and receive the supports they need to fulfill their potential with dignity, respect and equality" [5]. Furthermore, one of the major issue is the "balance between cure and care for PWD and their caregivers" utilizing knowledge, best practice and experience to improve dementia prevention, risk reduction, care and support. Additionally, it is necessary to generate new knowledge towards finding diseasemodifying treatments, as well as effective risk reduction's interventions and innovative models of care [5]. Dementia is one of major causes of disability and dependency among the elderly [6]. It is associated with the worst health outcomes [6]. Very often, clinicians delay the diagnosis of heart or pulmonary illness in these patients because PwD are unable to explain their symptoms appropriately [7]. Dementia is not only a problem of forgetting. Behavioral and psychological disorders (BPSDs) occur in most patients [8]. BPSDs can cause immense patient suffering and are responsible for both their hospitalization or institutionalization and caregiver distress [9-10]. Identification of predisposing and precipitating factors is very important. High rates of emergency department attendance could indicate inadequate availability of care in the community, a paucity of advance directives, or lack of focus on patients' quality of life rather than to an acute illness [11]. In fact, the emergency department becomes the only "escape" for unbearable situations at home or in nursing home [10-11]. While a good quality of life is the aim in caring for PwD, effective methods for evaluating this outcome are lacking. Moreover, dementia leads to the increased longterm costs for governments, communities, families and individuals, and loss in productivity for economies [4]. In 2015, dementia costs were estimated at US\$818 billion; by 2030, this is expected to reach the US\$2 trillion, a total that could undermine social and economic development and overwhelm health and social care systems $[4,5]$. Coping with this emergency, it is important to have available a qualified and integrated network home care and health services. In theory, the global dementia plan describes an ambitious perspective for a timely identification of this disease while providing the interventions to integrate for supporting dementia dyad (PWD and their caregiver) [5]. The notion of critical or integrated care pathway (ICP) has been used in different health settings [12-14]. The European Pathway Association defines a care pathway as "a complex of intervention for the mutual decision-making and organization of care process for a well-defined group of patients during a well-defined period" [12]. It is important to have an outline of anticipated care for dementia given that its trajectory is variable but ineluctable. It is essential to have a multidisciplinary team in an appropriate framework with the aim to support PwD and their family through the clinical experience. An uncertain illness trajectory and the unpredictable levels of cognitive deterioration, both of them typify dementia's syndrome; in this context, a dementia care pathway may be attractive. The dementia care pathway developed in England by the National Institute of Health and Clinical Excellence (NICE) has its starting point in the staff training. These guidelines represent a holistic support for practitioners [15]. The NICE pathway emphasizes the post-diagnostic phase promoting choices that support caregiver at home and maintaining as longer as possible the patient's independence [15]. Commonly, these pathways were built upon the scientific evidence. Nevertheless, they do not enjoy worldwide distribution because the ideal 
path for treating dementia often has to deal with local bureaucratic problems. The term pathway could suggest a roadmap where patients and their caregivers are placed on and where clinicians and health providers could have the total control. In our opinion, we consider it rather as a "net" made up by clinical, social and political providers (Figure 1). The purpose of this ICP is, firstly, to diagnose cognitive impairment promptly; secondly, to intercept the future needs that every patient could face. Although these aspects are shared and well accepted both by politicians and care providers, at the best of our knowledge, there are not some robust evidence in the operationalization of this revolutionary mission for dementia healthcare beyond the diagnosis moment. A lack of evidence about the ICP experiences means that we do not know how their idealized version could be managed in real practice. We need to determine the care trajectory that could improve the quality of life of these patients, and this is the priority of any political intervention.

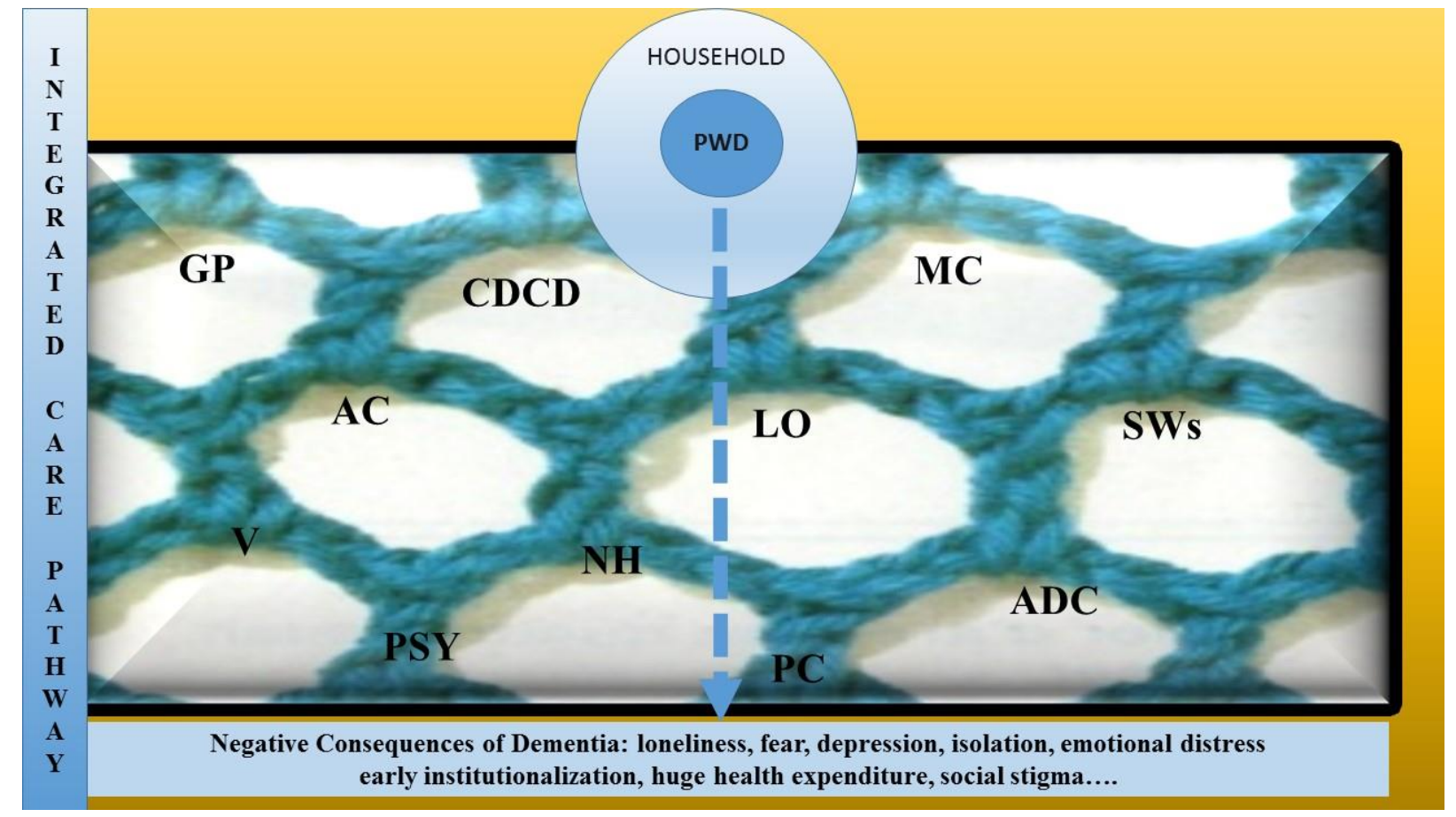

Figure 1 PWD, People With Dementia, GP, General Practitioner, CDCD, Center For Diagnosis Of Cognitive Disorders, MC, Multiservice Center, LO, Legal Office, SWs, Social Workers, V, Volunteers, PSY, Psychologists, AC, Alzheimer's Café, NH, Nursing Home, PC, Palliative Care, ADC, Adult Day Care.

In this article, we describe the operational protocol about the Clinical Pathway for PwD (named the Diagnostic-Therapeutic \& Healthcare Pathway for PwD specifically). It has been set up in the "Carlo Poma" Hospital, in Mantua, a city in the Northeast of Italy. Therefore, the purpose of this article is to present how we have planned the support network for PwD and their family in order to meet the National [16] and International [5] Guidelines for dementia.

In fact, the principal goals of this project are:

1) Facilitating the early diagnosis of cognitive disorder

2) Reducing the waiting time before to perform preliminary and specific tests 
3) Reducing the waiting time between the first visit and the formulation of diagnosis and starting therapy

4) Ensuring a good quality of life, preventing complications disease-related including family distress

5) Delaying institutionalization

6) Decreasing the access to the emergency department for BPSD management

7) Reducing the hospitalization rate

8) Creating a global service (point of reference for patients, their families, their caregivers, general practitioners (GPs) and other colleagues in acute departments where sometime PwD are admitted for other medical/surgical conditions).

\section{Materials and Methods}

A literature review was performed by searching the following terms "dementia care", "dementia plan", "dementia network", "dementia strategies", "dementia caring" on PubMed, Ovid, and ResearchGate. In addition, articles from pertinent sources, such as Italian government's reports and related website especially for local health legislature, were included in our search. We reported our project according to the SQUIRE Guidelines V.2.0, (Standard for Quality Improvement Reporting Excellence) [17]. A panel of 11 experts (neurologists, social workers, radiologists, neuropsychologist and statisticians of "Carlo Poma Hospital") has planned this protocol. For validation of preliminary draft, a modified Delphi technique was employed [18]. The responsible of Quality Management Office of our hospital verified the final draft that finally was approved by the Head Health Manager. This protocol was implemented in our Center for Diagnosis of Cognitive Disorders (CDCD) at the Neurological Department of our hospital, with data collection starting from 1 July 2017. Furthermore, data will be analyzed, and findings will be reported after a period of at least two years. An improvement group (with representatives of the entire professional area involved in this project) was set up to address the upcoming critical aspects during the application of the protocol.

After four Delphi rounds, five topical moments in this pathway have reached a consensus (Table 1):

1. Early symptoms identification and first service encounters

2. Assessment process

3. Diagnostic disclosure and Integration of Treatment's Plan

4. Follow-ups

5. Post-diagnostic support and appropriate interventions until the end-life stage.

We have enclosed in this protocol several care professional: experts who work in the CDCD (neurologists, geriatricians and neuropsychologists), a trained nurse who represents the case officer of $C D C D$, social workers, and legal representatives. The CDCD staff works closely with the Multiservice Center (MC). $M C$ is a multidisciplinary service that help people living with precarious conditions (including PwD) to resolve social, financial and administrative problems. In fact, thanks to the cooperation among nurses, physical therapists and protective services from municipal district, the MC has the possibility to activate readily helpful services (for example the Adult Day center, nursing home, residential care) and other social measure to support any difficulties that every patients and their family could have during the disease's course. 
Table 1 Integrated care pathway for people with dementia and their families: Principal phases.

\begin{tabular}{ll}
\hline Phase $\mathbf{1}$ & Suspicion, first diagnostic check, specialist evaluation. \\
\hline Phase $\mathbf{2}$ & Diagnosis and starting treatment. \\
\hline Phase $\mathbf{3}$ & Communication, training and support for caregiver and families. \\
\hline Phase $\mathbf{4}$ & Follow-up: easy paths that allow reducing dropout \\
\hline Phase $\mathbf{5}$ & $\begin{array}{l}\text { Post-diagnosis: Monitoring the patient at home for BPSD onset } \\
\text { (vouching and verification by the GP/specialist, communication } \\
\text { network among hospital, specialists, center for dementia, nursing } \\
\text { home adult day center for People with Cognitive Disorders) }\end{array}$ \\
\hline
\end{tabular}

Three psychologists with a proved experience in dementia field will administer standardized and normed psychological tests. Based on compendia of cognitive testing $[19,20]$ and the recommendations from the Italian Neuropsychological Society [21], the psychologists will perform a comprehensive cognitive assessment-Italian version for all enrolled patients (Table 2). Furthermore, the functional status and the social and family load of dementia will be evaluated too (Table 2).

Table 2 Global assessment for people with dementia.

\begin{tabular}{|c|c|}
\hline Psycho-cognitive Domains & Assessment test \\
\hline screening test & MINI MENTAL STATE EXAMINATION [22] \\
\hline $\begin{array}{l}\text { Screening of a global } \\
\text { executive dysfunction }\end{array}$ & FRONTAL ASSESSMENT BATTERY [23] \\
\hline Working memory & DIGIT SPAN TASK /BLOCK SPAN TASK [24] \\
\hline Episodic memory & 15-WORD LIST-LEARNING TEST [25] \\
\hline attention & DIGIT CANCELLATION TEST [26] \\
\hline executive functions & $\begin{array}{l}\text { MATRIX TEST [27] } \\
\text { TRAIL MAKING TEST [28] }\end{array}$ \\
\hline Language & CATEGORY FLUENCY TEST LANGUAGE [29] \\
\hline Constructional abilities & IDEOMOTOR APRAXIA TEST [30] \\
\hline $\begin{array}{l}\text { emotional status and } \\
\text { behavior }\end{array}$ & NEUROPSYCHIATRIC INVENTORY (NPI) [31] \\
\hline FUNCTIONAL DOMAINS & $\begin{array}{l}\text { BASIC ACTIVITIES OF DAILY LIVING [32] } \\
\text { INSTRUMENTAL ACTIVITIES OF DAILY LIVING } \\
{[33]}\end{array}$ \\
\hline FAMILY & ADULT CARER QUALITY OF LIFE (AC-QOL) [34] \\
\hline DOMAINS & CAREGIVER BURDEN INVENTORY (CBI) [35] \\
\hline & DEMENTIA ATTITUDE SCALE [36] \\
\hline & RELATIVE STRESS SCALE (RSS) [37] \\
\hline & 7-ITEMS SOCIAL FRAILTY SCALE [38] \\
\hline
\end{tabular}


We have strengthened the role of general practitioners (GPs) as the first promoters of an initial assessment as well as of an early refer to the specialist consultant for any suspected case. For this purpose, we have organized some informative meetings to explain our project to all of our urban and non-urban districts GPs, In our project, the GPs have to perform the General Practitioner Cognitive Assessment (GP-Cog) test-Italian version [39] in persons with suspected cognitive impairment and in persons at increased risk of cognitive impairment. GP-Cog takes no longer than five minutes to administer this test that comprises two components: a six item cognitive assessment with the patient and an informant questionnaire if the cognitive assessment score is equivocal (between 5-8). Scores $>8$ are deemed to represent cognitive impairment and $<5$ intact cognition (sensitivity 82-85\%; specificity 83-86) [40]. GPs may contact the CDCD specialist both directly by an email or phone and indirectly by a communication to the MC. A nurse is always available to receive whichever questions or concerns from the users (GPs, patients, caregivers). We have also planned a monitoring chart to detect each variation of clinical status of patient and the improvement or not of caregiver distress during the period between the follow-ups. For this purpose, a nurse from MC was assigned to complete this monitoring chart by a phone interview to caregiver, especially when the specialist reports some situation at risk (distressful behavior, changes in therapy, delirium, high distress of caregiver and so on). Our project is oriented to manage both clinical and social condition of patients. In this respect, we formalized the principal situations that make necessary a close connection (by phone or email) between specialists, GPs and $\mathrm{MC}$ in order to counteract promptly all the emerging situations that alters the frail balance of our users (Table 3).

A trained nurse from the CDCD works closely with one nurse from the MC. They represent the interface between patients' needs and the clinical and social providers. In order to facilitate the access to dementia care pathway for our patients without any concerns from the caregiver, they book the appointments personally.

Table 3. Precipitating factors that make necessary a close connection among the ICP principal clinical providers (General Practitioner, Specialist, Multiservice Center).

\begin{tabular}{ll}
\hline Drug-related emergency & Side effects, inefficacy, drug-drug interaction \\
\hline $\begin{array}{l}\text { BPSD onset or their } \\
\text { worsening }\end{array}$ & $\begin{array}{l}\text { Wandering, agitation, including delirium } \\
\text { superimposed dementia ... }\end{array}$ \\
New medical conditions & $\begin{array}{l}\text { Blood hypertension, hypo/hyperglycemia, flu, } \\
\text { hospitalization, falls.... }\end{array}$ \\
A family or social changes & \begin{tabular}{l} 
Bereavement, caregiver's illness, financial change \\
\hline
\end{tabular}
\end{tabular}

\subsection{The Screening Moment}

With a GP-Cog score pathological or borderline, GPs have to send a communication to the MC. Sometime people contact directly the private specialists for a visit. In addition, other specialists may contact the $M C$ indicating the name of patient who could benefit for a second level of intervention. GPs, specialists from other hospitals and citizens may consult a webpage site of our hospital to contact the staff [41]. Alternatively, after a first neurological evaluation or 
hospitalization for other causes, people at high suspicious of cognitive impairment are addressed to the CDCD (for example, patients who developed delirium, or showed clear sign of cognitive impairment but without a previous diagnosis of dementia) $[42,43]$.

\subsection{The Referral Moment}

This is the step where patients and their family are enrolled in this ICP. Initially, the case officer of CDCD (she is a trained nurse who works in the Neurological Department) schedules the clinical visit for patient and their family. She cope with every problem that PwD and their family may present into access to ICP. Patients have to perform all the planned investigations depending on the clinical suspicion in according to the International Guidelines on Dementia (blood tests, instrumental examinations, and neuropsychological assessment); subsequently, the case officer makes an appointment directly avoiding further passages for caregivers in other offices. In Italy, in fact, a center for reservation of medical appointments fixes the date depending on the free place on agenda without considering the case priority.

\subsection{The Diagnostic Moment}

In addition to the basal investigations, our neuropsychologists perform a comprehensive neuropsychological assessment. The neuropsychologists produce a comprehensive report (a detailed history and two to three hours of paper-and-pencil tests) for the family and the CDCD ' $s$ specialist, offering specific recommendations for further treatment or timing for re-evaluation. After this, the nurse from MC contact patients for the booked specialist consultation (neurologist or geriatrician with a proven experience in the field of dementia) who decides if there are need to other investigations or it is possible to start with therapy. At this point, the social workers in our group intervene to assist the caregivers in order to plan useful measures able to support them (open home, respite care, voucher for municipal assistance). Moreover, they explain the legal consequence of this disease.

\subsection{The Follow-Ups}

We have standardized the timing of follow-ups (respectively at 1, 3 and 6 month after the diagnostic moment). These appointments are useful for clinicians to check the pharmacological effects and to monitor the clinical condition and the variation about the abilities to live independently. Moreover, they can also intercept every problem in disease management at home. In fact, at the end of each follow-up, an e-mail summarizing the final report is sent from the case officer of CDCD to the specialist consultant and the MC contemporaneously. This communication allows us to know the updated situation promptly.

\subsection{Post-Diagnostic Moment}

After the first six months, clinicians and neuropsychologists re-evaluate the patients' history and disease course. In this way, it is possible to organize specific strategies and programs. Sometime, we need to launch pro-active policies to support the vulnerability of the whole household. Actually, this last phase depends a lot on the disease's stage. In fact, if a patient starts its pathway when he/she is in the middle or late stage of dementia, any social, protective and pro- 
active measures have to be realized as soon as possible, often at the beginning of ICP or concurrent with the clinical appointments.

\subsection{Caregivers' Support}

During the appointment with the specialist, the MC staff give instructions to the caregiver about some measures that could be implemented at home. Likewise, they seek to increase the awareness of the necessity to activate a legal protection, drawing up a social and caring profile. MC staff also perform a triage schedule that includes the assessment scale for social frailty and caregiver distress (Table 1). In particular, the seven-item social frailty index and the Relative Stress Scale (RSS). The first assesses some items like living arrangements, education, socioeconomic status, social network, and support providing a score between " 0 " and "7", with higher scores indicating higher levels of social frailty.

RSS covers emotional distress, restrictions on the caregivers' social life and negative feelings directed towards the patient. It consists of 15 items and each item is scored at five levels of intensity, from zero = 'not at all' to four = 'to a high degree'. Moreover, we also evaluated the caregiver distress by the Distress Scale of the Neuropsychiatric inventory (NPI-D). NPI-D measures the distress associated with the patient's BPSD. This test covers the 12 items of the NPI and is rated on a six-level scale ranging from 0 ("not at all") to five ("very severely or extremely"). The higher the sum score, the higher is the caregiver's distress. Finally, MC completes the personalized care plan identifying the case-care manager trained to monitor the situation of our patients at home.

Alongside this pathway, psychological support groups were established for caregivers. Aim of these groups were to reduce the negative impact once a diagnosis of dementia was made. These groups not only provides support but also helps to build the resilience process. Not only do they provide education about how to self-manage their loved one at home but also about financial support whom they can benefit from regional or governmental measures.

\subsection{Monitoring System}

A computer technology company has implemented some software for our hospital. This software allows reporting when PWD is admitted to emergency room or in other medical or surgical department (the name of this program is Healthcare Portal that enters in the Electronic Informative Healthcare System of our region) [44]. In fact, when PwD is admitted into the emergency department for other medical conditions, the electronic triage sheet provides a symbol to identify people belonging to ICP and they are reported quickly to the MC. This latter, then, will inform the CDCD's specialist in order to evaluate the patient during the hospitalization, avoiding the delirium onset or other complications where it is possible. Furthermore, MC activates the procedures for a prompt discharge at home or in other community services with the necessary devices (i.e., wheelchairs).

To report social needs of these patients (especially in the hospital discharge moment), we use a computerized notification form (the MAntua Intranet Ats, MAIA system) [14] so that the professional figures working in the primary care may know in real time the needs for PwD at home. 
The "Carlo Poma" ethics committee approved this project; then, a referent belonging to the Research Quality Association verified the protocol formulation. The code of this protocol is PDTA 70.

\section{Discussion}

Dementia is a de-structuring disease that affects the individual abilities to live independently and to relate itself with other people. Our "Diagnostic-Therapeutic \& Healthcare Pathway for PwD" is an operative integrated care protocol that was born in order to create a multi-professional network able to support PWD and their families. This ICP has been developed inside of an acute setting, that is, in the principal hospital of our district (the Carlo Poma Hospital, in Mantua, Lombardy, Italy). In this hospital, a dedicated staff coordinates multiple services coping with any emerging needs of our patients. In this way, we wanted to help PwD and their families to survive to the terrible consequences of this disease including its negative impact on social life (Figure 1). The Carlo Poma Hospital has both a mission and vision. The mission is to compete with all the other stakeholders on the provision of essential assistance levels, in order to protect and promote physical and mental health. Furthermore, it works to ensure a safe life for all of the elderly living with dementia in their life context, through a model of integrated assistance between hospital and community. Furthermore, it works to ensure a safe life for all of the elderly living with dementia in their life context, through a model of integrated assistance between hospital and community. The vision of our company is "from treatment to take care", especially of chronic and fragile patients and their families. The promotion of participation and valorization of volunteering is encouraged too. In line with the international literature, our hospital recognizes the importance to have a family and community support for $\operatorname{PwD}[46,47]$. For this reason, a MC works for a full involvement of family and community fighting the dangerous stigma linked to dementia.

The principal goal of our protocol was to create a multi-component system that provides a quick and satisfactory access to clinical services. By a standardized re-evaluation of clinical, psychological and social conditions and a personalized care planning, we want to resolve issues related to the emergent problems in the dementia's course. This aspect is very important because it identifies timely any underlying problems (physical, cognitive behavioral and social changes). Recently, the transitional care for people with chronic disease has gained great importance [48]. Caring for PwD in acute setting is challenging especially when the acute phase (see for example pneumonia infection) is overcome and the patients must be discharged. Often their family are frightened about the care load after an acute event that worsen the previous frail balance of their loved one. For this reason, it is mandatory to define new and appropriate modality to manage some fundamental phases that are still fragmentary and not sufficiently coordinated. After reaching a complete consensus, our experts have identified the crucial phases in the disease management of persons living with cognitive impairment (Table 1). This project has integrated different professional figures and departments for an articulated intervention plan. It is important to raise awareness in social community and among GPs who are the first detector of any suspicious change in the cognitive performance of our patients. As dementia is a progressive chronic condition that impair the ability to live independently, it is reasonable to assume that PwD keep close contact with their own GP firstly [49]. In fact, family physicians are generally patients' first point of contact with the health system. Further, they are positioned ideally to provide care 
for individuals living with dementia from the early to the end stages of the illness [50, 51]. Sometimes, the caregiver's perception does not go in this direction [52]. This is why we pressed that specialists or social workers in the acute setting to keep contact with the GP constantly.

Dementia is a dyad-disease because it affects both patients and their family. The size of the problem, however, is such that a broad approach is needed. A comprehensive response by the health and social care system (both public and private) and other government sectors is mandatory. Moreover, we are convinced that a more and structured dementia care pathway should be integrated in the health community. In fact, it is important to provide some comprehensive, multisector policy responses to improve quality of life and reduce stigma and social isolation for PwD and their caregivers.

Another important aspect is that the residential living or day care for PwD often are expensive. The number of sufferers was estimated to increase more and more and the retirement benefits probably will not cover the health cost entirely (especially in some country as in Italy where politic situation has influenced the household economic power hitting the weakest groups of our society). Consequently, to meet these needs and demands, it will be important to develop centers that could coordinate any aspect of this multifaceted disease (both the clinical and the social features).

In our project, the $\mathrm{MC}$ will be responsible to maintain partnership with Adult day care, Alzheimer's Café, and with the municipal social workers. Social networks are important in the prevention and in the management of dementia [53]. A center staffed by clinical providers, social workers, and volunteers could offer an opportunity for leisure activities and social support to PwD. In fact, several studies have demonstrated the usefulness of a variety of non-pharmacological approaches to reduce the negative effects of giving care to PwD. Nevertheless, the current scientific evidence is conflicting. Sometimes, caregivers provide care with little or no support [5456], while suffering from poor health themselves [56]. In fact, family members who provide care to individuals with chronic or disabling conditions are themselves at risk. Emotional, mental, and physical health problems arise from complex caregiving situations [57]. For this reason, it is necessary to consider the importance of psychological groups for caregiver support. Brodaty and colleagues stated that most caregivers are satisfied with the educational programs they have attended reporting an improvement in their relationship with the patient [58]. Based on this evidence, we have established some psychological family groups with two meetings monthly. We collaborate with territorial services, volunteers, association groups to grow-up the social networks promoting the social activities. In our project, PwD are not excluded from the own social life and we try to avoid the social stigma of this disease.

Dementia is a standing concern around the world. Despite convincing evidence on the pathogenesis and epidemiology of dementia, so far we know little about the experiences of people with cognitive disorders in the health system and how the health system is modeled on them. Some examples of dementia care pathway $[15,59]$ such as the Dementia Care Pathway in New Zealand [60] and in Australia [61] provided an attempt to meet the needs of PwD. They offer a guidance across various services but only recommendations for acute care. By the evaluation of the current practice, they identified barriers and facilitators for the implementation of the best practice in dementia care. However, there is not a coordinated and integrated vision to join acute, chronic and community setting as in our project. Some studies explored the current state of health assistance for older people with dementia in the acute care setting but without mention to the integrated care pathway. Although there was an increase in the development and use of these 
pathways in the acute settings [62-64], however some concerns have emerged. In fact, there is still an insufficient evidence to support the effectiveness of these pathways in the acute setting and more studies should be encouraged.

This project has several strengths. Firstly, the possibility in our acute setting to keep close contact with various agencies and department in care provision. Secondly, the possibility to create a definite safety net for PWD and their family by the simultaneous activation of more interventions. Potentially, this could prevent some possible breakdowns in the healthcare system. In fact, because dementia is a chronic, disabling and devastating disease, it affects the healthcare expenditure enormously, especially where there is not a priori care program. Thirdly, our pathway assesses, engages, and supports caregivers (Figure 1). It is well-known that every intervention performed in order to reduce the caregiver's distress influence the behavior and well-being of PwD positively [65]. For this reason, we wanted to provide to caregivers effective tools to reduce their distress through psychological support groups, explaining with clarity what could happen during the life course in PwD. Moreover, we give them the possibility to keep close contact with the CDCD (they can write in any moment to the CDCD's specialist).

Finally, we believe that communication between clinical providers is essential to intercept early cognitive impairment cases. We have promoted an open approach to the health system through a close involvement of general practitioners. In fact, general practitioners are the first doctor with whom people deal with their health problems. However, gaps in symptom recognition and initiation of cognitive tests could lead to diagnostic delay or improper interpretation. Frequently, there is no communication between care providers, specialists and primary care physicians. This lack of connection is a weaker link to disease detection [66]. Hospitals are full of dementia screening resources, but time constraints and serious medical problems are preventing an efficient diagnosis of dementia. The memory clinics offer access to multidisciplinary skills (neurologist, geriatric and neuropsychologist), demonstrating an early diagnosis of dementia and a potential cost-effectiveness, but they are disadvantaged by long waiting lists. Once the diagnosis is made, it is necessary to follow the PWD, but sometimes it is not easy to have timely access to clinical services. Dementia is a very common and increasing disease and it is very important to create a multiple network that includes general practitioners, hospitals for acute illnesses, specialized clinics, communities and nursing homes. Governments have been called to provide good quality care and services for PWD. Countries were asked to shape and share solutions to provide facilities through the continuum of health care. Furthermore, it is mandatory to ensure both the development and training of a harmonized workforce able to counteract the huge impact of this disease.

\section{Conclusions}

The aging population, the changes in household structure, the increase of immigration rate (linked to a new class of elderly in the future), and the economic crisis, all of these are contributing factors that must encourage to develop and implement of strategies for welfare support. Despite an extensive research in the field of dementia, deficits in the quality of dementia care still exist. There is a paucity of robust research concerning the care experiences for PwD, although several countries have yet to implement the ICPs in their settings. The interpretation of existing ICPs requires careful consideration and cautious judgements on their suitability and feasibility. In our 
opinion, an economic evaluation and a better definition of outcomes could enhance the quality and the spreading out of this care pathway for PwD. It is mandatory to promote these experiences if we want to analyze some impactful policies improving services for PwD and their family.

Although the international and national guidelines drive how the care trajectory should be, unfortunately at the local level the correct procedures are often missed because the bureaucratic problems and the paucity of resources interfere with the guidelines application. In fact, without a well-structured, real based action plan against dementia we could assist to a collapse of our healthcare system. It follows that the local governments might encounter in the real life different limitations to the application of the ideal project, so we need to share and cope with them; otherwise, we are going to fail into manage the dementia syndrome over time. Where no cure is still available, we have to bet on the care system.

Actually, the true problem is a lack of organization among clinical and social providers who have to co-operate for the same goal, that is, to ensure the best, personalized and appropriate pathway of care for PwD. However, providers struggle with some problems as the time constraints, coordination of care, and a limited clinical support system, often times resulting in care outcomes that are less than personally desirable and satisfying.

Implementing dementia care pathway in the hospital setting, as shown in our experience, is a potential means of improving care, enabling existing services to work in a more unified way. This operational protocol set up the interventions to enable the healthcare system providing true and qualified care for PwD. Everyone belonging to the ICP will receive supports and treatments that are targeted to meet its individual requirements. Furthermore, thanks to a whole cooperation of all above-mentioned actors in this pathway, it is possible to improve the transition between community and acute setting for PWD. Whether the ICP for dementia is a useful method for the care process or not, it must still be evaluated. Therefore, it is very important to promote further studies centered on PwD that explore the best method for ethical and sustainable health use. Our future efforts are heading in this direction.

\section{Acknowledgments}

We want to thank the research team of "Dementia Care Pathway" of ASST "Carlo-Poma" Hospital and all of the associations and Nursing Home that help us to give the best support to our patients and their families.

We want to thank Savina from "Fondazione Mons. Arrigo Mazzali - ONLUS" together to all colleagues that are working there.

We thank Dr. Suheil Abdul Ponnambath for his help in English revision of our manuscript

Finally, we thanks our patients and their families because they give us a challenge to cope with every day.

\section{Author Contributions}

V. Frisardi, contributed to the analysis of the results and to writing of the manuscript.

F. Biagi, E. Galante, A. Balzanelli, E. Talassi and M.C Cilia perform the neuropsychological assessments of patients and provide to data collection. 
G. Capiluppi, she is a nurse of the MC and she contributed to help GP and people to have an easy access to dementia care pathway; she filled out evaluation scale for monitoring patients at home.

L. Frittoli is a case officer of CDCD and she contributed to enroll the patients and to keep close contact with them, Further, she encouraged V Frisardi to rationalize this project and supervised the findings of this work.

D. Terzi; E.Podavini G. Gazzoni, G. Simoncelli have cooperated with V. Frisardi and they supervised the findings of this work.

S Faroni, A. Taragnani, worked to promote this project and ameliorated the dementia care pathway.

C Matarazzo, M Avesani, C.M Stucchi discussed the results and contributed to visit patients.

A. Ciccone, Balzanelli A. Ventura A., Terzi D. Taragnani A, Bellani, Galante E, Biagi F, Talassi E, C. Basili, M.Galavotti, contributed to the design and implementation of the research.

\section{Funding}

This project is inserted in the national plan for dementia.

\section{Competing Interests}

The authors have declared that no competing interests exist.

\section{References}

1. Connolly A, Gaehl E, Martin H, Morris J, Purandare N. Underdiagnosis of dementia in primary care: Variations in the observed prevalence and comparisons to the expected prevalence. Aging Ment Health. 2011; 15: 978-984

2. World Alzheimer Report [WHO] 2015 The Global Impact of Dementia. Available from: www.worldalzreport2015.org/downloads/world-alzheimer-report-2015.

3. Niu H, Álvarez-Álvarez I, Guillén-Grima F, Aguinaga-Ontoso I. Prevalence and incidence of Alzheimer's disease in Europe: A meta-analysis. Neurologia. 2017; 32: 523-532.

4. Alzheimer's Disease International. Policy Brief for Heads Of Government. The global Impact of Dementia 2013-2050. London. Available from: http://www.alz.co.uk/research/GloballmpacDementia2013. October 14,2014.

5. Global action plan on the public health response to dementia $2017-2025$. WHO. Available from: http://www.who.int/mental_health/neurology/dementia/action_plan_2017_2025/en/

6. Agüero-Torres H., Fratiglioni L, Guo Z, Viitanen M, von Strauss E, Winblad B. Dementia is the major cause of functional dependence in the elderly: 3-year follow-up data from a population-based study. Am J Public Health. 1998; 88: 1452-1456.

7. Skinner T, Scott I, Martin J. Diagnostic errors in older patients: A systematic review of incidence and potential causes in seven prevalent diseases Int J Gen Med. 2016; 9: 137-146.

8. Hope T, Keene J, Fairburn CG, Jacoby R, McShane R. Natural history of behavioural changes and psychiatric symptoms in Alzheimer's disease. A longitudinal study. Br J Psychiatry. 1999; 174: 39-44. 
9. Maust DT, Kales HC, McCammon RJ, Blow FC, Leggett A, Langa KM. Distress associated with dementia-related psychosis and agitation in relation to healthcare utilization and costs. Am J Geriatr Psychiatry. 2017; 25: 1074-1082.

10. Sampson EL, White N, Leurent B, Scott S, Lord K, Round J, et al. Behavioural and psychiatric symptoms in people with dementia admitted to the acute hospital: Prospective cohort study. Br J Psychiatry. 2014; 205: 189-196.

11. Sleeman KE, Perera G, Stewart R, Higginson IJ. Predictors of emergency department attendance by people with dementia in their last year of life: Retrospective cohort study using linked clinical and administrative data. Alzheimers Dement. 2018; 14: 20-27.

12. Lillo-Crespo M, Riquelme J, Macrae R, De Abreu W, Hanson E, Holmerova I, et al. Experiences of advanced dementia care in seven European countries: Implications for educating the workforce. Glob Health Action. 2018; 11: 1478686.

13. Schrijvers G, van Hoorn A, Huiskes N. The care pathway: Concepts and theories: An introduction. Int J Integr Care. 2012; 12: e192.

14. Vanhaecht K, Panella M, van Zelm R, Sermeus M. An overview on the history and concept of care pathways as complex interventions. SAGE. 2010; 14: 117-123.

15. National Institute of Clinical Excellence Pathways. Dementia: Supporting people with dementia and their carers in health and social care. Available from: https://www.nice.org.uk/guidance/cg42.

16. Di Fiandra T, Canevelli M, Di Pucchio A, Vanacore N. Italian dementia national plan working group. The Italian dementia national plan. Commentary. Ann Ist Super Sanita. 2015; 51: 261264.

17. Goodman D, Ogrinc G, Davies L, Baker GR, Barnsteiner J, Foster TC, et al. Explanation and elaboration of the SQUIRE (Standards for Quality Improvement Reporting Excellence) Guidelines, V.2.0: examples of SQUIRE elements in the healthcare improvement literature. BMJ Qual Saf. 2016; 25: e7.

18. Keeney $\mathrm{S}$, Hasson $\mathrm{F}$, Mckenna $\mathrm{H}$. The delphi technique in nursing and health research. West Sussex: John Wiley \& Sons Ltd; 2010.

19. Lezak M, Howieson D, Loring D. Neuropsychological assessment. New York: Oxford University Press; 2004.

20. Strauss E, Sherman E, Spreen O. A compendium of neuropsychological tests. New York: Oxford University Press; 2006.

21. Barletta-Rodolfi C, Gasparini F, Ghidoni E. Kit del neuropsicologo italiano. Milano: Dynamicon; 2011.

22. MF Folstein, SE Folstein, PR McHugh. Mini Mental State Examination. J Psychiat Res. 1975; 12 : 189-198.

23. Appollonio I, Leone $M$, Isella $V$, Piamarta $F$, Consoli $T$, Villa $M L$, et al. The frontal assessment battery (FAB): Normative values in an Italian population sample. Neurol Sci. 2005; 26: 108116.

24. Monaco M, Costa A, Caltagirone C, Carlesimo GA. Forward and backward span for verbal and visuo-spatial data: Standardization and normative data from an Italian adult population. Neurol Sci. 2013; 34: 749-754.

25. Rey A. L'examen clinique en psychologie. Paris: Presses Universitaires de France; 1964. 
26. Della Sala S, Laiacona M, Spinnler H, Ubezio C. A cancellation test: Its reliability in assessing attentional deficits in Alzheimer's disease. Psychol Med. 1992; 22: 885-901.

27. Abbate C, Luzzatti C. Vergani C. Matrix test: Speed and accuracy of visual search in aging. G Gerontol. 2007; 55: 11-20.

28. Giovagnoli AR, Del Pesce M, Mascheroni S, Simoncelli M, Laiacona M, Capitani E. Trail making test: Normative values from 287 normal adult controls. Ital J Neurol Sci. 1996; 17: 305-309.

29. Costa A, Bagoj E, Monaco M, Zabberoni S, De Rosa S, Papantonio AM, et al. Standardization and normative data obtained in the Italian population for a new verbal fluency instrument, the phonemic/semantic alternate fluency test. Neurol Sci. 2014; 35: 365-372.

30. Tessari A, Tornalo A, Lunardelli A, Zadini A, Rumiati RI. STIMA: A short screening test for ideomotor apraxia, selective for action meaning and bodily district. Neurol Sci. 2015; 36: 977-984.

31. Cummings JL, Mega M, Gray K, Rosemberg-Thompson S, Carusi DA, Gornbei J. Neuropsychiatric Inventory (NPI). Neurology. 1994; 44: 2308-2314.

32. Katz S., Akpom C. A. Index of ADL. Med Care. 1976; 14: 116-118.

33. Lawton MP. Instrumental activities of daily living. Gerontologist. 1969; 9: 179.

34. Negri L, Piazza G, Sartori RDG, Cocchi MG, Delle Fave A. The adult carer quality of life questionnaire (AC-QoL): Comparison with measures of burden and well-being, and Italian validation. Disabil Rehabil. 2018; 10: 1-10.

35. Novak M, Guest C. Application of a multidimensional caregiver burden inventory. Gerontologist. 1989; 29: 798-803.

36. O'Connor ML, McFadden SH. Development and psychometric validation of the dementia attitudes scale international. Int J Alzheimers Dis. 2010; 2010: 454218.

37. Greene JG, Smith R, Gardiner M, Timbury GC. Measuring behavioural disturbance of elderly demented patients in the community and its effects on relatives: a factor analytic study. Age Ageing. 1982; 11: 121-126.

38. Teo N, Gao Q, Nyunt MSZ, Wee SL, Ng TP. Social frailty and functional disability: findings from the Singapore longitudinal ageing studies. J Am Med Dir Assoc. 2017; 18: 637.

39. Pirani A, Brodaty $H$, Martini E, Zaccherini D, Neviani F, Neri M. The validation of the Italian version of the GPCOG (GPCOG-It): A contribution to cross-national implementation of a screening test for dementia in general practice. Int Psychogeriatr. 2010; 22: 82-90.

40. Yokomizo JE, Simon SS, Bottino CM. Cognitive screening for dementia in primary care: A systematic review. Int Psychogeriatr. 2014; 26: 1783-1804.

41. Multiservice center of ASST Mantova. Available from: http://www.asst-mantova.it/centromultiservizi.

42. Cole MG. Delirium in elderly patients. Am J Geriatr Psychiatry. 2004; 12: 7-21.

43. Inouye SK. Cognitive decline: Strategies for prevention: Proceedings of a white house conference on aging. London: Greenwich Medical Media; 1997.

44. Electronic Informative Healthcare System of ASST Carlo Poma. Available from: http://www.siss.regione.lombardia.it/wps/portal/Minisiti/siss.

45. A Measurement and Performances Rating System. MAntua Intranet Ats (MAIA) System website. Available from: https://www.aslmn.net/

46. Jing $W$, Willis $R$, Feng $Z$. Factors influencing quality of life of elderly people with dementia and care implications: A systematic review. Arch Gerontol Geriatr. 2016; 66: 23-41. 
47. Herron RV, Rosenberg MW. "Not there yet": Examining community support from the perspective of people with dementia and their partners in care. Soc Sci Med. 2017; 173: 8187.

48. Ray CA, Ingram V, Cohen-Mansfield J. Systematic review of planned care transitions for persons with dementia. Neurodegener Dis Manag. 2015; 5: 317-331.

49. Moore A, Frank C, Larry WC. Role of the family physician in dementia care. Can Fam Physician. 2018; 64: 717-719.

50. Moore A. Fourth canadian consensus conference on the diagnosis and treatment of dementia. Recommendations for family Physicians. Can Fam Physician. 2014; 60: 433-438.

51. Villars H, Oustric S, Andrieu S, Baeyens JP, Bernabei R, Brodaty $\mathrm{H}$, et al. The primary care physician and Alzheimer's disease: an international position paper. J Nutr Health Aging. 2010; 14: $110-120$

52. Schoenmakers B, Buntinx F, Delepeleire J. What is the role of the general practitioner towards the family caregiver of a community-dwelling demented relative? A systematic literature review. Scand J Prim Health Care. 2009; 27: 31-40.

53. Fratiglioni L, Paillard-Borg S, Winblad B. An active and socially integrated lifestyle in late life might protect against dementia. Lancet Neurol. 2004; 3: 343-353.

54. Alzheimer's Association \& National Alliance for Caregiving. Families Care: Alzheimer's Caregiving in the United States. Chicago: Alzheimer's Association and Bethesda; 2004.

55. Family Caregiver Alliance Caregiver Assessment: Principles, Guidelines and Strategies for Change. Report from a National Consensus Development Conference (Vol. I). (2006).

56. Navaie-Waliser M, Feldman PH, Gould DA, Levine CL, Kuerbis AN, Donelan K. When the caregiver needs care: The plight of vulnerable caregivers. Am J Public Health. 2002; 92: 409413.

57. Pearlin LI, Mullan JT, Semple SJ, Skaff MM. Caregiving and the stress process: An overview of concepts and their measures. Gerontologist. 1990; 30: 583-594.

58. Brodaty $\mathrm{H}, \mathrm{Green} A$, Koschera A. Meta-analysis of psychosocial intervention for caregivers of people with dementia. J Am Geriatr Soc. 2003; 51: 657-664.

59. Dementia Strategy NHS Forth Valley. Available from: https://nhsforthvalley.com/.../Dementia-Strategy-2017-2020

60. New Zealand Framework for Dementia Care - Ministry of Health. Available from: https://www.health.govt.nz/publication/new-zealand-framework-dementia-care

61. Tropea J, LoGiudice D, Brand C. The Dementia Care Pathway for use in acute care. Available from: $\quad$ https://medicine.unimelb.edu.au/research-groups/medicine-and-radiologyresearch/royal-melbourne-hospital/melbourne-epicentre/the-dementia-care-pathway-foruse-in-acute-care.

62. Moyle W, Borbasi S, Wallis M, Olorenshaw R, Gracia N. Acute care management of older people with dementia: a qualitative perspective. J Clin Nurs. 2011; 20: 420-428.

63. Butcher L. Caring for patients with dementia in the acute care setting. Br J Nurs. 2018; 27 : 358-362.

64. Handley M, Bunn F, Goodman C. Dementia-friendly interventions to improve the care of people living with dementia admitted to hospitals: a realist review. BMJ Open. 2017; 7: e015257. 
65. Feast A, Moniz-Cook E, Stoner C, Charlesworth G, Orrell M. A systematic review of the relationship between behavioral and psychological symptoms (BPSD) and caregiver wellbeing. Int Psychogeriatr. 201; 28: 1761-1774.

66. Marshall M, Phillips D. A qualitative study of the professional relationship between family physicians and hospital specialists. Prof Geogr. 1999; 51: 274-282.

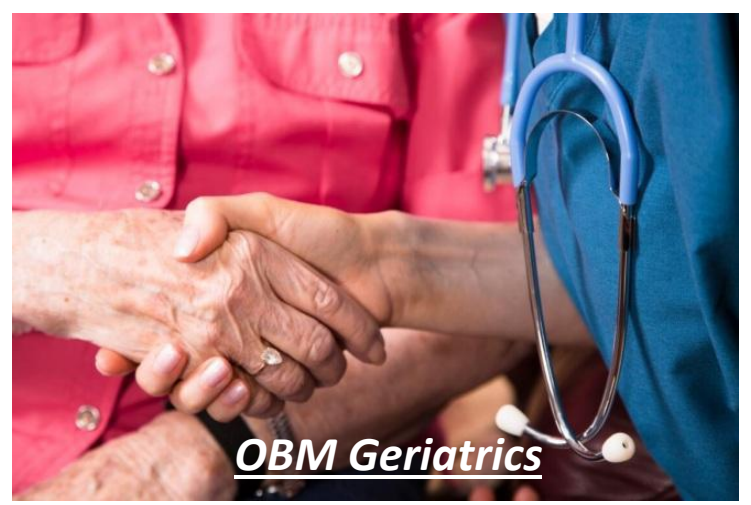

Enjoy OBM Geriatrics by:

1. Submitting a manuscript

2. Joining in volunteer reviewer bank

3. Joining Editorial Board

4. Guest editing a special issue

For more details, please visit: http://www.lidsen.com/journals/geriatrics 
Hypothesis

Sporadic Alzheimer Disease and That Developing in Down's Syndrome: The Immune System Attacking Self Rather Than Suppressing Infectious Disease Invaders, Toll Like Receptors Triggering Excessive Cytosolic Calcium, Excess Calcineurin Activation, Overexpression of Regulator of Calcineurin1, Runaway Beta-Amyloid Production, Synaptic Loss, Destructive Inflammation and Dementia

Steven R. Brenner *

Retired Neurologist, Formerly St. Louis University and the St. Louis VA hospital, MO, USA; E-Mail: SBren20979@aol.com

* Correspondence: Steven R. Brenner; E-Mail: SBren20979@aol.com

Academic Editor: Michael Fossel

OBM Geriatrics

2019, volume 3, issue 1

doi:10.21926/obm.geriatr.1901035
Received: August 29, 2018

Accepted: February 13, 2019

Published: February 26, 2019

\begin{abstract}
Alzheimer Disease may result from excessive stimulation of the innate immune system from development of underlying opportunistic infections and impaired age related selfrecognition as non-self, due to immunodeficiency and immunosenescence, resulting in excessive inflammation and runaway Beta-amyloid production (a component of the innate immune system) causing cytosolic calcium overload. Excessive cytosolic calcium may cause over activation of calcineurin and inactivation of cis-trans prolyl isomerase (Pin1), with subsequent loss of dendritic spine maintenance, and synaptic destruction.

Improving immune function, identifying and treating infections, avoiding runaway Betaamyloid production, inhibiting calcineurin in a manner similar to that utilized in preventing tissue transplant rejection, maintaining the negative feedback loop between regulators of calcineurin1 (RCAN1) and calcineurin, may lead to improved prevention and treatment of Alzheimer Disease and Down's syndrome.
\end{abstract}

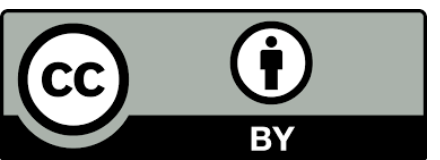

(C) 2019 by the author. This is an open access article distributed under the conditions of the Creative Commons by Attribution License, which permits unrestricted use, distribution, and reproduction in any medium or format, provided the original work is correctly cited. 


\section{Keywords}

Alzheimer's disease; dementia; Down's syndrome; immune; aging; inflammation; infection; synapse; calcineurin; beta-amyloid

\section{Introduction}

Alzheimer's disease (AD) continues to be a debilitating condition, despite enormous efforts to develop treatments, based primarily on the Beta-Amyloid hypothesis. Further research has indicated the likelihood of infectious disease activation, possibly due to failure of the immune system to recognize foreign invaders, and instead attacks self in ways similar to that which occurs in transplant biology.

Immunosuppression, Immuno-toxicity, immunosenescence and Opportunistic Infections: Opportunistic infections may occur from immune suppression and immune-toxicity due to multiple agents including metal dyshomeostasis, from excess free copper [1], zinc deficiency [2], iron overload [3], aluminum accumulation [4] cytomegalovirus (CMV) encoded interleukin-10 [5], diabetes and obesity [6] which have been noted to be related to AD. Aging, the main risk factor for $A D$ development, is associated with immunosenescence related to declining adaptive immunity involving $T$ and $B$ cells [7]. These abnormalities result in immunosuppression and/or altered immune function [7-12]. Such infections from viruses, bacteria or fungi stimulate Beta-amyloid production in the brain, Beta-amyloid being an anti-microbial peptide and a normal component of the innate immune system [13]. Evidence of viruses, bacteria and fungi have been detected in brains of $A D$ patients, indicating $A D$ may arise in response to infections [14]. Human herpes virus $6 \mathrm{~A}$ and 7 were identified in $A D$ patients when compared to controls in three independent cohorts dispersed geographically with regulatory relationships between viral abundance and Amyloid Precursor Protein metabolism indicating a viral relationship with molecular, clinical and neuropathological features of AD [15], Cytomegalovirus, Human herpes virus 2, bacteria (including spirochetes and periodontal pathogens such as Porphyromonas gingivalis and Treponema denticola) [5], and fungi [16] have all been identified in AD brain examinations. Porphyromonas gingivalis especially is of interest since it is able to modulate the host immune response through virulence factors facilitating development of chronic periodontitis with involvement of additional oral flora constituents [17]. Modulating the immune response leads to a form of localized immunosuppression in the periodontal region.

P. gingivalis lipopolysaccharide also circulates systemically in more than half of periodontitis patients causing inflammation with higher levels of matrix metalloproteinase-9 [18], metalloproteinase-9 being elevated in AD patients [19]. Beta-amyloid appears to be an antimicrobial peptide, or natural antibiotic produced by the brain in response to infections as a component of the innate immune system [13], rather than a spontaneous development leading to AD. This may be the reason efforts to reduce Beta-amyloid production have not prevented AD. Recurrent or progressive opportunistic infections, especially in a person with some degree of immunosuppression, may lead to increased Beta-amyloid production exceeding clearance. Excessive Beta-amyloid production could lead to loss of anti-microbial function through runaway domain swap, and runaway Beta-amyloid production or other mechanisms of polypeptide 
aggregation into a pathogenic Beta-amyloid form [20]. If there are recurrent opportunistic infections in $A D$ brain, reducing or eliminating Beta-amyloid production could possibly even worsen the condition. This is essentially the "Amyloid Protection Hypothesis" of AD, put forward by Tanzi [21] in that Beta-Amyloid is an anciently conserved effector molecule of innate immunity, enabling entrapment of pathogens to protect from infection. Increased levels of brain microbes may stimulate Beta-amyloid production and deposition, leading to inflammation and $A D$ progression[21].

Innate Immune System Activation and Inflammation: Innate immune system activation by recurrent or chronic infections arising due to immunodeficiency activates toll like receptors (TLR), causes acute and chronic inflammation, similar to tissue rejection, as occurs in allogeneic organ transplants. TLR's mobilize intracellular calcium [22], causing calcium dependent calcineurin, to be activated. Calcineurin negatively regulates toll like receptor 2 and 4 activation [23]. Patients on calcineurin inhibitors having a decreased cytokine response to toll like receptor signaling [22]. Excessive Calcineurin activation appears to be an early stage in the pathogenesis of AD and solid organ transplant patients on the calcineurin inhibitor, FK506 had a much lower incidence of AD than expected [24]. ABeta42 also activates calcineurin through post-synaptic signaling, which in turn dephosphorylates (deactivates) Pin1, which maintains dendritic spines [24], leading to loss of synapses as happens in AD. Further validation that calcineurin is involed in Beta-amyloid mediated synaptic loss involves experimental evidence from transgenetically modified plaque bearing mice and wild type mice as well, in which the calcineurin inhibitor, FK-506 (tacrolimus), ameliorated plaque related synaptic loss [25].

Additional features involved with synaptic loss involve complement and microglia, complement possibly being activated by microglia in $A D$ as well, being observed in AD mouse models, since C1q, the initiating protein in classical complement cascade or CR3, the microglial complement receptor interacts with and is necessary for the toxic effects of Abeta oligomers on synapses and hippocampal long term potentiation [26]. Microglia in adult brains engulfs synaptic material involving the microglial complement receptor CR3, when exposed to soluble Abeta. This is suggestive of inappropriate reactivation of developmental pathways in $A D$, which prune excessive synapses during development, but eliminate essential synapses in AD. Increased expression of beta-amyloid precursor protein APP 695, a specific isoform containing 695 amino acids, does occur during neuronal differentiation of primary hippocampal neurons in a cell culture system, indicating differentiation of neurons is accompanied by increased Beta-APP695 expression and membrane retention of the APP695 protein [27]. The Amyloid Precursor Protein is also a receptor for Slit, a protein involved in axonal guidance during development [28]. This may indicate Beta-Amyloid is moonlighting, or during development, Amyloid precursor protein is involved in neuronal development with axonal guidance, leading to synaptic pruning, which occurs during brain development.

Prefibrillary or oligomeric abeta also appears to induce calcineurin activity, which mediates its effects on neuronal cells [29]. Pathological loss of Pin1 has been found in synapses of AD cortical brain specimens, and in dendritic rafts and post-synaptic density. Pin1 controls cell cycle progression and degrading proteins through the ubiquitin proteasome system, which could result in the accumulation of misfolded Beta-amyloid plaques and hyperphosphorylated tau tangles years, if not decades prior to development of clinical disease as well as altered post synaptic density proteins such as Shank protein involved in organization which would cause distorted 
synaptic structures, increased susceptibility to toxicity and impaired synaptic plasticity [30]. Pin1 is a member of a number of a family of molecular chaperones regulating protein folding at proline residues. The microtubule-associated protein, tau, has abundant proline residues. Members of the Peptidyl-prolyl cis/trans isomerase family interact with and regulate tau. Besides Pin1, FK506binding protein (FKBP) 52, FKBP51, and FKBP12 interact with and regulate tau, which is hyperphosphorylated in AD. Pin1, FKBP52, FKBP12, cyclophilin A, CyclophilinB and CyclophilinD also regulate $A B$ eta production or $A B$ eta toxicity in $A D$ [30]. A cancer related variant of a cell cycle inhibitor gene p21Cip1 is associated with a greater risk of AD [31]. The increased susceptibility to infections in the elderly is mainly due to decreased efficiency of adaptive immunity to suppress microorganisms [32]. Alzheimer patients may also experience a greater degree of immunosenescence than the healthy elderly.

Alzheimer patients have been found to have decreased numbers of CD3+T cells in peripheral blood, when compared to non-demented subjects, the cells themselves exhibiting increased protein oxidation and nitrosative stress when levels of 3-nitrotyrosine are assessed, the proteins being involved in energy metabolism, cytoskeletal structure, intracellular signaling, protein turnover and folding and antioxidant response [33].

Regulator of calcineurin(RCAN1) discovered as a gene on chromosome 21 in Down's syndrome, or Trisomy 21, is overexpressed in Down's syndrome (frequently associated with early onset AD), and also is linked to pancreatic beta-cell dysfunction in type 2 diabetes. Its functions are primarily to inhibit calcineurin and mitochondrial function. Reactive oxygen species, hyperglycemia and other forms of cellular stress cause transient increase in expression. Short term expression causes protective effects through the calcineurin/NFAT pathway, however long term expression over weeks and months causes pathological changes in cells, such as those seen in Down's syndrome, $A D$, and type 2 diabetes [34]. It's interesting that Beta-amyloid is expressed, not only in the brain, but also the pancreas in type 2 diabetes [35].

\section{Conclusions}

It appears multiple opportunistic infections in an immune-compromised and/or immunosuppressed state, as well as aberrant innate immune system activation against self rather than non-self, cause excessive stimulation of the innate immune system through TLR's, resulting in excessive if not runaway Beta-amyloid production. Both Beta-amyloid and recurrent infections cause extensive triggering of TLR's on aged neurons, resulting in increased cytosolic $\mathrm{Ca}^{2+}$, calcineurin activation with subsequent loss of Pin1 activity, loss of dendritic spines, synaptic loss, and likely death. Microglia is also likely activated causing widespread excessive inflammation.

Inflammation, neuronal death, and synaptic loss are all features of AD. Efforts to increase immunity and avoid infections may be instrumental in both prevention and treatment of AD. Calcineurin inhibitors, such as FK506, as suggested by O'Neal, et al [24] may be a useful approach in treatment of $A D$ as well, and could be helpful in prevention of synaptic loss from immunosensenescence, or excessive inflammation resulting from the aged immune system attacking self. Immunosensecence leading to a miss-targeted immune system, (permitting infections while attacking self) likely interacts in development of age related diseases such as AD, and other age related conditions. Intervention in multiple aspects of $A D$ development and 
progression may be necessary to achieve success, such as reducing pathogenic oral flora, improving overall immunity, and suppressing aberrant immune responses.

Diet may have some role as well, since diets rich in foods containing polyphenols with nuts, soy, citrus, berries, olive oil, tea, leafy vegetables and red wine seem to reduce the chance of developing $A D$ [36]. Polyphenols may have some role in reducing bacterial growth in the oral flora and intestinal biome [37]. Reducing the impact of age related diseases such as AD may depend on enhancing immunological memory through methods such as vaccination and suppressing aberrant innate immune response against self, likely in a manner similar to preventing rejection in tissue transplants. With immunosensescence, immunotolerance may have been lost. Restoring immunotolerance calls for suppression of aberrant innate and adaptive immunity, similarly to the approach taken in treating allografts. Medicaitons such as mycophenolate may be helpful in this regard as well as the calcineurin inhibitors.

Besides calcineurin, chronic overexpression of regulator of calcineurin (RCAN1) as occurs in Down's Syndrome/Trisomy 21, also referred to as Down's Syndrome Critical region 1 (DSCR1) which is encoded on chromosome 21, also occurs in AD. RCAN1 expression is up-regulated in aging, the greatest risk factor for late onset $A D$, and also by Beta-amyloid [38]. Beta-amyloid is secreted or shed in response to microbial infection in the CNS, likely due to immunosensecence related to aging, possibly from latent $\mathrm{CMV}$, as well as reactivation of latent Herpes viruses. This sequence of events in the elderly recapitulates the events in Trisomy 21, which almost invariably leads to onset of $A D$ at an earlier age than usually presents in late onset $A D$.

While a negative feedback loop for calcineurin and DSCR1/RCAN1 is present, it is likely overwhelmed in AD and Trisomy 21 [39]. People with the ApoE genotype epsilon4/epsilon4, a high-risk genotype for developing AD have higher levels of RCAN1 in lymphocytes than people with lower risk genotypes. RCAN1 upregulates glycogen synthase, a tau kinase, which would increase tau phosphorylation when RCAN1 is upregulated, hyperphosphorylated tau being characteristic of AD. Beta-amyloid causes such upregulation, so RCAN1 links the two hallmarks of $A D$, Beta-amyloid in Alzheimer plaques and hyperphosphorylated tau in neurofibrillary tangles [40]. It may be possible to lessen the effects of RCAN1 overexpression through dietary measures.

This has been investigated in an experimental study involving fish oil supplement provided to mice, and there was a modest reduction of RCAN1 RNA and protein following such dietary intervention. It was thought such a dietary intervention for persons with Down's syndrome might have some benefit [41]. In a study involving neuronal cell culture, lycopene reduced adverse effects of RCAN1 through reducing oxidative stress and apoptosis, indicating a diet including lycopene- containing foods could have beneficial effects for persons with Down's syndrome or AD [42]. Considering that calcinerin and RCAN1 regulate calcium and the interrelationship of calcium and vitamin $D$, vitamin $D$ status likely has involvement in AD risk.

In a study involving vitamin D levels in older adults, deficient vitamin D levels were associated with nearly a three times higher risk of developing $A D$ [43]. In a comparison of $A D$ incidence between the Monongahela Valley of Pennsylvania, USA, and Ballabgarh, India, the incidence was practically three times higher in the Pennsylvania cohort compared to the cohort in India. Being at higher latitude, the Pennsylvania location would have lower sunshine exposure, and so could possibly have a lower vitamin D status than the lower latitude cohort in Ballabgarh, India [44]. Probably in prevention and management of $A D$, and also Down's syndrome, avoiding calcium overload by maintaining the negative feedback mechanism between RCAN1 and calcineurin 
expression and function is a prime consideration. It may be possible to assess whether the relationship between RCAN1 and calcineurin is optimal through metabolic studies of peripheral blood cells, through assessment of RCAN1 expression level. AD being a multifactorial disease, it's unlikely that one approach to management will fit all.

Further investigations involving immunosenescence and its causes will be helpful in understanding if it has a relationship to pathogenesis of $A D$ and the basic causes of aging, which likely involve additional fields such as epigenetics, activity and exercise, susceptibility to infections and the many aspects of calcium metabolism and signaling.

\section{Author Contributions}

The author made all contributions to this work.

\section{Competing Interests}

The author has declared that no competing interests exist.

\section{References}

1. Squitti R, Ghidoni R, Simonelli I, Ivanova ID, Colabufo NA, Zuin M, et al. Copper dyshomeostasis in Wilson disease and Alzheimer's disease as shown by serum and urine copper indicators. J. Trace Elem Med Biol . 2018; 45: 181-188.

2. Ventriglia M, Brewer GJ, Simonelli I, Mariani S, Siotto M, Bucossi S, et al. Zinc in Alzheimer's disease: a meta-analysis of serum, plasma, and cerebrospinal fluid studies. J Alzheimers Dis. 2015; 46: 75-87.

3. Tao Y, Wang Y, Rogers JT, Wang F. Perturbed iron distribution in Alzheimer's disease serum, cerebrospinal fluid, and selected brain regions: a systematic review and meta-analysis. J Alzheimers Dis. 2014; 42: 679-690.

4. Zapatero M, de Jalon AG, Pascual F, Calvo M, Escanero J, Marro A. Serum aluminum levels in Alzheimer's disease and other senile dementias. Biol Trace Elem Res. 1995; 47: 235-240.

5. Sochocka M, Zwolinska K, Leszek J. The infectious etiology of Alzheimer's Disease. Curr Neuropharmacol. 2017; 15: 996-1009.

6. Mayeux R, Stern Y. Epidemiology of Alzheimer disease. Cold Spring Harb Perspect Med. 2012: a006239.

7. Nikolich-Žugich J. Aging of the T cell compartment in mice and humans: from no naive expectations to foggy memories. J Immunol. 2014; 193: 2622-2629.

8. Keswani T, Mitra S, Bhattacharyya A. Copper-induced immunotoxicity involves cell cycle arrest and cell death in the liver. Environ Toxicol. 2015; 30: 411-421.

9. Cunningham-Rundles S, McNeeley DF, Moon A. Mechanisms of nutrient modulation of the immune response. J Allergy Clin Immunol. 2005; 115: 1119-1128.

10. Cherayil BJ. Iron and immunity: immunological consequences of iron deficiency and overload. Arch Immunol Ther Exp. 2010; 58: 407-415.

11. Zhu Y, Hu C, Li X, Shao B, Sun H, Zhao H, et al. Suppressive effects of aluminum trichloride on the T lymphocyte immune function of rats. Food Chem Toxicol. 2012; 50: 532-535. 
12. Spencer JV, Lockridge KM, Barry PA, Lin G, Tsang $M$, Penfold $M E$, et al. Potent immunosuppressive activities of cytomegalovirus-encoded interleukin-10. J Virol. 2002; 76: 1285-1292.

13. Soscia SJ, Kirby JE, Washicosky KJ, Tucker SM, Ingelsson M, Hyman B, et al. The Alzheimer's disease-associated amyloid $\beta$-protein is an antimicrobial peptide. Plos One. 2010; 5: e9505.

14. Fulop T, Witkowski JM, Bourgade K, Khalil A, Zerif E, Larbi A, et al. Can an infection hypothesis explain the beta amyloid hypothesis of Alzheimer's disease? Front Aging Neurosci. 2018; 10.

15. Readhead B, Haure-Mirande J-V, Funk CC, Richards MA, Shannon P, Haroutunian V, et al. Multiscale analysis of independent Alzheimer's cohorts finds disruption of molecular, genetic, and clinical networks by human herpesvirus. Neuron. 2018.

16. Pisa D, Alonso R, Rábano A, Rodal I, Carrasco L. Different brain regions are infected with fungi in Alzheimer's disease. Sci Rep. 2015; 5: 15015.

17. How KY, Song KP, Chan KG. Porphyromonas gingivalis: an overview of periodontopathic pathogen below the gum line. Front. Microbiol. 2016; 7: 53.

18. DeLeon-Pennell KY, de Castro Brás LE, Lindsey ML. Circulating Porphyromonas gingivalis lipopolysaccharide resets cardiac homeostasis in mice through a matrix metalloproteinase-9dependent mechanism. Physiol Rep. 2013; 1.

19. Lorenzl S, Albers DS, Relkin N, Ngyuen T, Hilgenberg SL, Chirichigno J, et al. Increased plasma levels of matrix metalloproteinase-9 in patients with Alzheimer's disease. Neurchem Int. 2003; 43: 191-196.

20. Khare SD, Dokholyan NV. Molecular mechanisms of polypeptide aggregation in human diseases. Curr Protein Pept Sci. 2007; 8: 573-579.

21. Moir RD, Lathe R, Tanzi RE. The antimicrobial protection hypothesis of Alzheimer's disease. Alzheimers Dement. 2018; 14: 1602-1614.

22. Ren $H$, Teng $Y$, Tan $B$, Zhang $X$, Jiang $W$, Liu $M$, et al. TLR triggered calcium mobilization protects mice against bacterial infection through extracellular ATP release. Infect Immun. 2014: IAI. 02546-02514.

23. Kang YJ, Kusler B, Otsuka $M$, Hughes $M$, Suzuki $N$, Suzuki $S$, et al. Calcineurin negatively regulates TLR-mediated activation pathways. J Immunol. 2007; 179: 4598-4607.

24. O'Neal MA, Stallings NR, Malter JS. Alzheimer's Disease, Dendritic Spines, and Calcineurin Inhibitors: A New Approach? : ACS Publications; 2018.

25. Rozkalne A, Hyman BT, Spires-Jones TL. Calcineurin inhibition with FK506 ameliorates dendritic spine density deficits in plaque-bearing Alzheimer model mice. Neurobiol Dis. 2011; 41: 650-654.

26. Hong S, Beja-Glasser VF, Nfonoyim BM, Frouin A, Li S, Ramakrishnan S, et al. Complement and microglia mediate early synapse loss in Alzheimer mouse models. Science. 2016; 352: 712-716.

27. Hung $A Y, K o o E H$, Haass $C$, Selkoe DJ. Increased expression of beta-amyloid precursor protein during neuronal differentiation is not accompanied by secretory cleavage. Proc Natl Acad Sci. 1992; 89: 9439-9443.

28. Wang B, Li H, Ozseker SA, Bowser DA, Moore MJ, Wang MC, et al. The amyloid precursor protein is a conserved receptor for slit to mediate axon guidance. eNeuro. 2017: 0185-0117.

29. Reese LC, Zhang W, Dineley KT, Kayed R, Taglialatela G. Selective induction of calcineurin activity and signaling by oligomeric amyloid beta. Aging cell. 2008; 7: 824-835. 
30. Blair LJ, Baker JD, Sabbagh JJ, Dickey CA. The emerging role of peptidyl-prolyl isomerase chaperones in tau oligomerization, amyloid processing, and Alzheimer's disease. J Neurochem. 2015; 133: 1-13.

31. Yates SC, Zafar A, Rabai EM, Foxall JB, Nagy S, Morrison KE, et al. The effects of two polymorphisms on p21cip1 function and their association with Alzheimer's disease in a population of European descent. Plos One. 2015; 10: e0114050.

32. Castaneda-Delgado J, Miranda-Castro N, Gonzalez-Amaro R, Gonzalez-Curiel I, MontoyaRosales A, Rivas-Calderon $B$, et al. Production of antimicrobial peptides is preserved in aging. Clin Immunol. 2013; 148: 198-205.

33. Tramutola A, Abate G, Lanzillotta C, Triani F, Barone E, lavarone F, et al. Protein nitration profile of $\mathrm{CD} 3+$ lymphocytes from Alzheimer disease patients: Novel hints on immunosenescence and biomarker detection. Free Radic Biol Med. 2018; 129: 430-439.

34. Peiris $\mathrm{H}$, Keating DJ. The neuronal and endocrine roles of RCAN 1 in health and disease. Clin Exp Pharmacol Phsiol. 2018; 45: 377-383.

35. Miklossy J, Qing H, Radenovic A, Kis A, Vileno B, Làszló F, et al. Beta amyloid and hyperphosphorylated tau deposits in the pancreas in type 2 diabetes. Neurobiol Aging. 2010; 31: 1503-1515.

36. Lefèvre-Arbogast S, Gaudout D, Bensalem J, Letenneur L, Dartigues J-F, Hejblum BP, et al. Pattern of polyphenol intake and the long-term risk of dementia in older persons. Neurology. 2018: 10; 1212.

37. Brenner SR. Reader Response: Polyphenols in the diet may inhibit development of Alzheimer disease by antibiotic effects on bacteria in the intestinal biome and oral flora. Neurology 2018.

38. Cook CN, Hejna MJ, Magnuson DJ, Lee JM. Expression of calcipressin1, an inhibitor of the phosphatase calcineurin, is altered with aging and Alzheimer's disease. J Alzheimers Dis. 2005; 8: 63-73.

39. Sun X, Wu Y, Herculano B, Song W. RCAN1 overexpression exacerbates calcium overloadinginduced neuronal apoptosis. Plos One. 2014; 9: e95471.

40. Lloret A, Badia M-C, Giraldo E, Ermak G, Alonso M-D, Pallardó FV, et al. Amyloid- $\beta$ toxicity and tau hyperphosphorylation are linked via RCAN1 in Alzheimer's disease. J Alzheimers Dis. 2011; 27: 701-709.

41. Zmijewski PA, Gao LY, Saxena AR, Chavannes NK, Hushmendy SF, Bhoiwala DL, et al. Fish oil improves gene targets of Down syndrome in C57BL and BALB/c mice. Nutr Res. 2015; 35: 440448.

42. Lim S, Hwang S, Yu JH, Lim JW, Kim H. Lycopene inhibits regulator of calcineurin 1-mediated apoptosis by reducing oxidative stress and down-regulating Nucling in neuronal cells. Mol Nutr Food Res. 2017; 61: 1600530.

43. Feart C, Helmer C, Merle B, Herrmann FR, Annweiler C, Dartigues J-F, et al. Associations of lower vitamin $\mathrm{D}$ concentrations with cognitive decline and long-term risk of dementia and Alzheimer's disease in older adults. Alzheimers Dement. 2017; 13: 1207-1216.

44. Chandra V, Pandav R, Dodge H, Johnston J, Belle S, DeKosky S, et al. Incidence of Alzheimer's disease in a rural community in India: the Indo-US study. Neurology. 2001; 57: 985-989. 


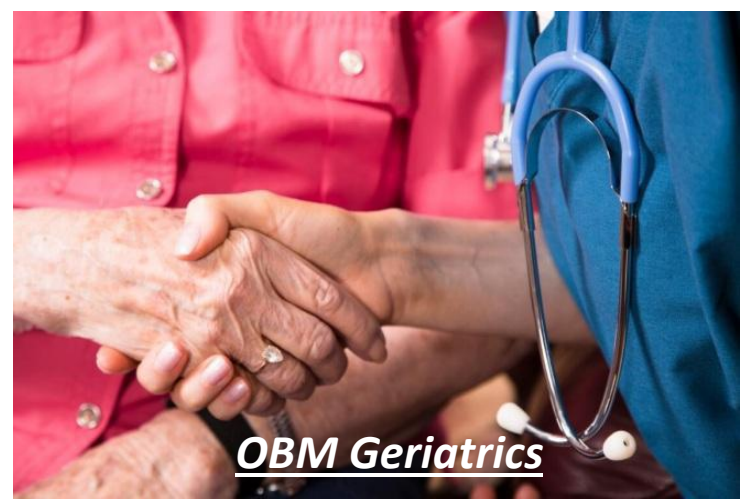

Enjoy $O B M$ Geriatrics by:

1. Submitting a manuscript

2. Joining in volunteer reviewer bank

3. Joining Editorial Board

4. Guest editing a special issue

For more details, please visit: http://www.lidsen.com/journals/geriatrics 
Review

\title{
Cell Senescence, Telomerase, and Senolytic Therapy
}

\author{
Michael Fossel $^{*}$
}

Telocyte LLC, 250 Monroe NE, Grand Rapids, MI, USA; E-Mail: Michael.fossel@telocyte.com

* Correspondence: Michael Fossel; E-Mail: Michael.fossel@telocyte.com

Academic Editor: James S. Powers

Special Issue: Perspectives on Telomeres and Aging

\author{
OBM Geriatrics \\ Received: December 31, 2018 \\ 2019, volume 3 , issue 1 \\ Accepted: January 31, 2019 \\ doi:10.21926/obm.geriatr.1901034 \\ Published: February 15, 2019
}

\begin{abstract}
The consensus that cell senescence plays a role in age-related disease has prompted a number of potential clinical interventions, including attempts to reset cell senescence and attempts to remove senescent cells from aging tissues. The latter approach, senolytic therapy, has attracted considerable attention, but both theoretical considerations and published data suggest that the clinical benefits will be transient and that senolytic therapies will likely accelerate long-term degenerative disease. We review the overall field, its history, the theoretical aspects, and the available data. The long-term risks are underestimated and based on naïve assumptions, while the long-term benefits are not borne out by physiologic considerations or data. Senolytics are likely to accelerate tissue pathology, exacerbate clinical disease, and result in early morbidity and mortality.
\end{abstract}

\section{Keywords}

Cell senescence; telomeres; telomerase; senolytics

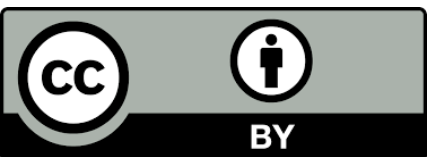

(C) 2019 by the author. This is an open access article distributed under the conditions of the Creative Commons by Attribution License, which permits unrestricted use, distribution, and reproduction in any medium or format, provided the original work is correctly cited. 


\section{Introduction}

Recent advances in our understanding of the role of senescent cells in age-related human disease have prompted several distinct interventional strategies, including:

1) Telomerase gene therapy to reset gene expression in senescent cells,

2) Small molecular drugs aimed at individual genes or proteins, and

3) Senolytic drugs to kill senescent cells.

These three approaches will be compared in light of human pathology and available data (generally animal data), with a focus on current data, as well as the clinical implications of senolytic therapy. A conceptual illustration of the point of intervention of each of these three approaches (and currently available symptomatic drugs), is shown here (Figure 1).

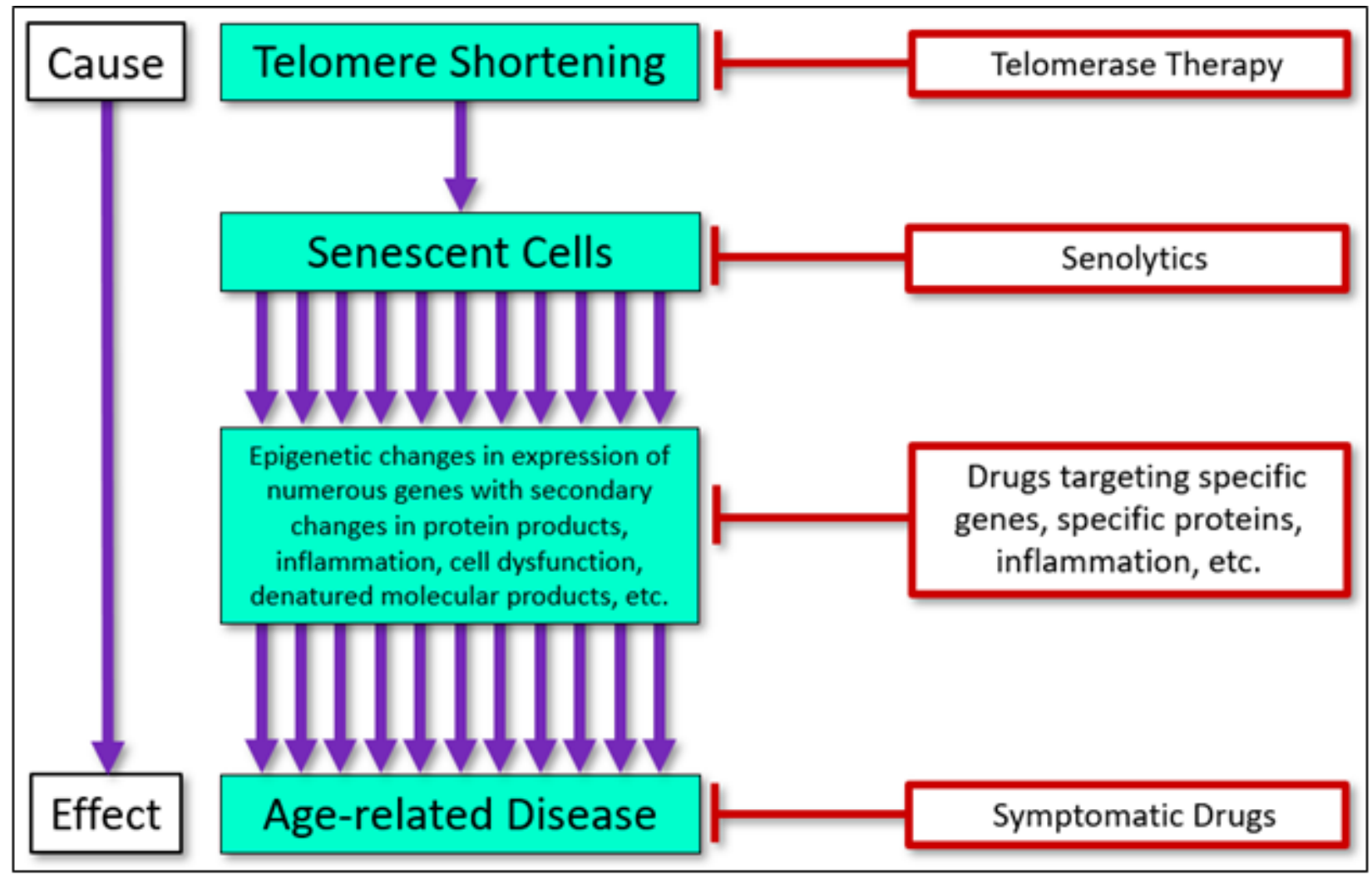

Figure 1 Targets and interventional approaches to age-related disease.

\section{Background}

Cell senescence was first demonstrated in the 1960's, predominantly due to the work of Hayflick and Moorhead [1, 2]. In 1990, the work of Harley and his colleagues at Geron demonstrated that cell senescence correlated closely with changes in telomere length [3], and this was followed by a series of confirmatory papers [4, 5]. Thereafter, the relationship between telomeres and cell senescence was shown to be not only correlational, but causal in a series of papers showing that resetting telomere lengths also reset cell senescence as measured by gene arrays, histology, and cell function [6-8].

The first articles in the medical literature $[9,10]$ as well as a textbook on the area [11], suggested that not only was cell senescence a key player in age-related human disease, but that 
cell senescence in general and telomeres in particular could serve as a uniquely effective point of clinical intervention. In the case of age-related vascular disease, for example, endothelial cell senescence was seen to precede the usual histologic hallmarks of atherosclerosis and do so in a manner that paralleled the locations and degree of pathology [12]. Similar findings were seen in other age-related diseases, such as osteoarthritis and osteoporosis, in which key cells (e.g., chondrocytes and osteocytes) showed senescent changes that preceded and appeared to cause the onset of clinical disease. Such senescent cells included vascular endothelial cells, chondrocytes, osteocytes, and other cell types in their respective tissues and organs.

Over the past two decades, and despite its obvious potential, pursuit of the potential of this initial work was limited by the available technology, which made it difficult to translate this work into in vivo animal or human studies, although some articles continued to clarify the model and point out the clinical possibilities. Specifically, additional experimental support for the model required genetic manipulations that became available as the field matured. In regard to human gene therapy, initial adverse events (e.g., the Jesse Gelsinger fatality in 1999) delayed progress and testing in human gene therapy trials.

Nonetheless, supportive animal and human data contined to accrue. With regard to the putative role of cell senescence in age-related disease, additional findings [13] suggested a similar process was occurring with the aging brain, specifically in glial cells, and that this process might underlie common clinical dementias, such as Alzheimer's type dementia [14]. Specifically, such glial cells demonstrate telomere shortening and a decreased ability to produce, bind, internalize, and breakdown key molecules, such as beta amyloid [15]. Moreover, glial cell senescence has been seen to precede neurofibrillary tangles and neurodegeneration [16]. Cell senescence is now seen as the cause, rather than the effect, of neural degeneration. As a 2018 editorial in Nature put it: "glial senescence ultimately promotes neuronal degeneration" [17]. The same view, that cell senescence is a causal factor not only in neuronal degeneration specifically, but in age-related diseases generally, is becoming the consensus view.

The first approach mentioned above, resetting gene expression, has worked well in animal studies [18, 19], and has solid theoretical support [11], but will not be addressed in detail here, although the use of telomerase to reset gene expression has the clear potential to reverse the disease course in age-related disease. The third approach, the use of small molecules, has shown limited efficacy: while effective in addressing specific genes and proteins, it does not address the broader panoply of changes in genes and proteins that characterize cell senescence and, in consequence, has shown no effect in altering the disease course in age-related disease. The classic example of this failure has been the use of monoclonal antibodies in Alzheimer's disease trials. The fourth approach, symptomatic therapies, not shown to affect the disease course of agerelated disease, is outside of both the interest and the scope of this paper.

The second approach, the use of senolytics will be evaluated here in some detail. We will consider the rationale for its use, the claims that have been made for its clinical potential, the data on its use, and implications of such data for both clinical use and economic value.

\section{Rationale for Senolytics}

Senescing (as well as fully senescent) cells become increasingly common in older tissues [20]. Such cells demonstrate multiple defining changes, including both generic (characteristic of 
senescent cells generally) and cell-specific changes (characteristic of specific tissue and cell types). Senescence is associated with telomere-modulated, characteristic changes in gene expression, termed SAGE (senescence associated gene expression). Senescing cells show pronounced intracellular changes including slower molecular turnover, slower DNA repair, deficient cell maintenance, defective mitochondrial function, less efficient ATP production (and a lower ATP/ROS ratio), increased percentages of accrued molecular damage, etc [11]. Nor is this increasing cellular dysfunction limited to intracellular venues. While senescing cells are known to become less effective in performing their own internal cellular functions, they also increasingly interfere in the functioning of other local, non-senescent cells. Bluntly, senescing cells not only cause tissue dysfunction by a passive loss of normal internal function, but they also cause tissue dysfunction by an active and external interference in the function of other cells. This process, termed SASP (senescence associated secretory phenotype) [21, 22] is generic to senescing cells in any tissue, with specific characteristics for individual cell and tissue type as well. It is characterized by the secretion of toxic molecules $[23,24]$, as well as by numerous molecular markers and changes in gene expression [25], including p16INK4A, and it can be identified by molecular markers [26], including SA- $\beta-$ Gal, inflammatory signalling molecules, growth factors and proteases [17].

To take a specific tissue as an example, the chondrocytes making up the bulk of any human joint surface senesce and demonstrate increasing internal cellular dysfunction as they do so. In addition, the dysfunction of such senescent cells actively interferes with the function of other neighboring non-senescent chondrocytic cells as well $[23,27]$. Osteoarthritis is the clinical result in an aging joint and is characterized by increasing inflammation, as well as the erosion and gross loss of normal joint surface. Current clinical interventions for osteoarthritis include drugs that are marginally or transiently effective (i.e., anti-inflammatory drugs, etc.) for symptomatic treatment or the complete removal and replacement of the affected joint (i.e., surgical replacement with an artificial joint). While replacement has become the orthopedic standard-of-care, it is expensive, painful, debilitating, inherently risky, and does not address the pathology per se. In short, current treatment options are sub-optimal. Generically, the same caveats pertain to all current interventions for age-related disease: current therapies have little-or-no effect upon the disease process itself and are clinically sub-optimal.

As a result, the three basic approaches listed above are all under consideration in the context of age-related disease. Currently available approaches intervene "downstream", at the level of clinical effects (symptoms) rather than "upstream" at the level of clinical causes (underlying disease processes). Again using the example of the aging joint and its chondrocytes, currently available therapeutic approaches do not address the underlying cause(s) of osteoarthritis, but merely serve to ameliorate symptoms or act by removing the aging tissue in toto. In contrast to current therapy, the three approaches listed above aim to address "upstream" processes at varying levels. Amplifying these three strategies, we may summarize each approach as follows:

1) At the telomere level: telomerase gene therapy aims to reset the telomere length, and hence gene expression, enabling the senescent cells to function normally and abrogating their interference with other, non-senescent cells. Here the goal is to fully restore normal cell and tissue function, restoring the joint to its previous, clinically normal state.

2) At the specific protein and gene level: small molecular drugs aim to individually target any and all characterized gene or molecular changes in the tissue, including cellular debris, 
mitochondrial changes, free radicals, cellular apoptosis, etc. While the goal is to mitigate the changes (particularly those of SASP), the sheer number of individual changes necessitates dozens of simultaneous interventions, none of which address the underlying cell senescence that drives such changes.

3) At the tissue level: senolytic therapy aims to remove senescent cells, mitigating or preventing SASP effects, and putatively enabling remaining, non- senescent cells to function more normally.

The last approach, removal of senescent cells, is generally termed "senolysis" in the medical and biotechnology literature, and putative agents are termed "senolytics". Initial data supported a potential value of senolytics $[28,29]$ and several biotechnology companies are currently engaged in promoting this approach [30]. Researchers and biotechnology companies have proclaimed a growing number of putative senolytic compounds, including AP20187 [27], ABT263 [31], INKATTAC [29], ABT-263 [32], FOXO4 peptide [33], UBX0101 and UBX1967 [34], and a growing list of others. This approach has garnered considerable interest in the research $[35,36]$ and clinical literature [28, 37], as well as among investors and the venture capital community.

\section{Claims Regarding Senolytics}

A number of articles have suggested that clearance of senescent cells can attenuate agerelated tissue changes (or age-related disease) and create a more favorable tissue environment $[36,38]$, and should therefore be considered appropriate for human clinical trials. Such articles accurately stress the likely role of senescent cells in age-related pathology, as well as the factors contributing to senescence, such as age per se, trauma, infection, etc. Published outcomes are generally supportive and intriguing, although (as discussed below) long-term effects are generally not evaluated $[23,29,31,33,38]$ or when long-term data appears in the publication, it is glossed over in favor of the initial positive effects, which are highlighted [27].

Such initially positive effects are consistent, as would be expected from the known effects of senescent cells in aging tissues, and these effects have been documented in several species and in different cell and tissue types. These cell and tissue types encompass several age-related pathologies [36], including tumorigenesis (as well as chemotherapy [33, 39]) and age-related deterioration in the kidney, heart, and fatty tissue [27], bone marrow and muscle [31], eye [29], brain [40], etc. These results have prompted further work on specific age-related diseases and prospective human trials (as well as considerable interest in the clinical and investment potential of these approaches).

In osteoarthritis, for example, there is good evidence that senescent cells accumulate in and parallel the course of joint pathology. Moreover, senolysis-attenuated post-traumatic osteoarthritis in mice in vivo and, human in vitro cultures taken from knee replacement patients, showed short-term benefits as assessed by inflammation and matrix protein formation. This suggests a potential benefit in human osteoarthritis [38].

In the case of vascular age-related disease, similar findings are seen (at least in mice), with senescent cells being common in atherosclerotic lesions, including foamy macrophages. Such results suggest that senescent cells play central roles in plaque formation, loss of elasticity, fibrous tissue, plaque rupture, and subsequent clots. The results imply a potential use for senolytic therapy in human atherosclerosis, although interventional data to support the suggestion is 
lacking [23]. Note that the role of cell senescence in human atherosclerosis was first noted more than two decades ago [12], as was its probable role in atherogenesis [8, 41-43]. The clinical potential for intervention in age-related arterial disease as well as other age-related disease by addressing cell senescence (using telomerase rather than senolysis) was also pointed out at that time $[9,10,44-46]$ and in more detail since [11].

Other proposed clinical targets include ophthalmologic disease as well as age-related diseases of the heart, kidney and liver [47]. Some authors have gone on to argue that the use of senolytics not only has benefits to treating the age-related pathology of specific tissues or organ systems, but will extend the healthy lifespan in animal models and would have similar effects in humans [27, 29, $31,48]$. Although articles advocating senolytic approaches occasionally note the possibility of acceleration of cell division in the remaining, initially non-senescent cells, no attention is given to the adverse consequences (enforced cell senescence of initially non- senescent cells) put into play by senolytic interventions [38].

\section{Adverse Effects of Senolytics}

Histological considerations suggest that senolytics will accelerate age-related disease in affected tissues. In a sense, this would be parallel to the known effects of radiation, which can induce cell senescence and reduce stem cell availability [49]. In the case of senolytics, the removal of senescent cells would accelerate stem cell division and consequent cell senescence, inducing premature cell and tissue aging, with subsequent acceleration of age-related clinical disease.

In any given tissue, there is a population of cells, some of which are senescent. If we remove cells (e.g., with senolytic therapy), the absence of those cells will trigger the remaining cells to divide in order to replace the cells which are no longer present. This standard, classsical histological response within any tissue replaces lost cells, as occurs with trauma to the dermis, routine epidermal turnover, the death and replacement of circulating lymphocytes, quotidian erythrocyte loss and replacement, hepatocyte turnover, etc. As these cells divide and replace cellular losses, their telomeres shorten and accelerate their progression toward cellular senescence. This acceleration of cell senescence, i.e., the division of the remaining cells in response to cell removal (in this case, the removal of senescent cells), has predictable negative consequences.

In the case of osteoarthritis, for example, while the goal is to entirely abrogate the effects of SASP, the use of senolytics not only removes senescent chondrocytes, but risks the acceleration of senescence in the remaining cells, which were initially non-senescent, as those remaining cells must now divide to replace the missing chondrocytes (i.e., those senescent chondrocytes removed by the use of senolytic agents). This has been seen in the case of osteoarthritis [38], for example, and has been attributed to a number of possible causes [36], including the underlying causes of cell senescence and the exhaustion of stem cells, as they replace the missing senescent cells.

As we remove senescent cells from an aging tissue, local factors will signal the nearby, remaining cells to divide and replace those cells which have been removed. The subsequent cell division results in two daughter cells. Both daughter cells lose telomere length, with the result that both daughter cells are themselves that much closer to senescence. The loss of senescent cells has momentarily resulted in a mean gain in telomere length in the remaining cells (and a mean loss of cell senescence and cell dysfunction), but as the remaining cells divide to replace the missing 
senescent cells, there is a mean loss of telomere length in the remaining cells, with consequent acceleration in cell senescence and cell dysfunction. We should expect that the initial improvement (as measured by telomere length, cell function, SASP, etc.) will be followed by more rapid deterioration (as measured by the same criteria). Removal of senescent cells should result in a transient gain in tissue function, followed by an accelerated loss of tissue function. Senolytic therapy threatens to transiently improve clinical status, while accelerating overall age-related clinical disease.

Consider the following graphic examples. In Figure 2, telomere length is on the $Y$ axis, while affected cells are shown on the $X$ axis of each of the four treatment phases. In the pre- treatment phase, we see three senescent cells, whose telomere lengths indicate cell senescence, resulting in tissue dysfunction. We also see six, non-senescent cells, whose telomere lengths indicate nonsenescence, thereby demonstrating normal cell function. When a senolytic treatment is applied, the immediate result is the deletion of senescent cells ("missing cells"), followed by signalling to neighboring cells ("stressed cells"), prompting the final result of cellular replication, telomere shortening, and an increased number of senescent cells. We now have six senescent daughter cells, whose telomere lengths are below the critical level and only three non- senescent cells, whose telomere lengths are above the same critical level. In this simplistic example, comparing pretreatment to the final result, we see that we have gone from $33 \%$ (3 of 9) senescent cells to $66 \%$ (6 of 9) senescent cells, thereby accelerating the very tissue pathology that we intended to treat.

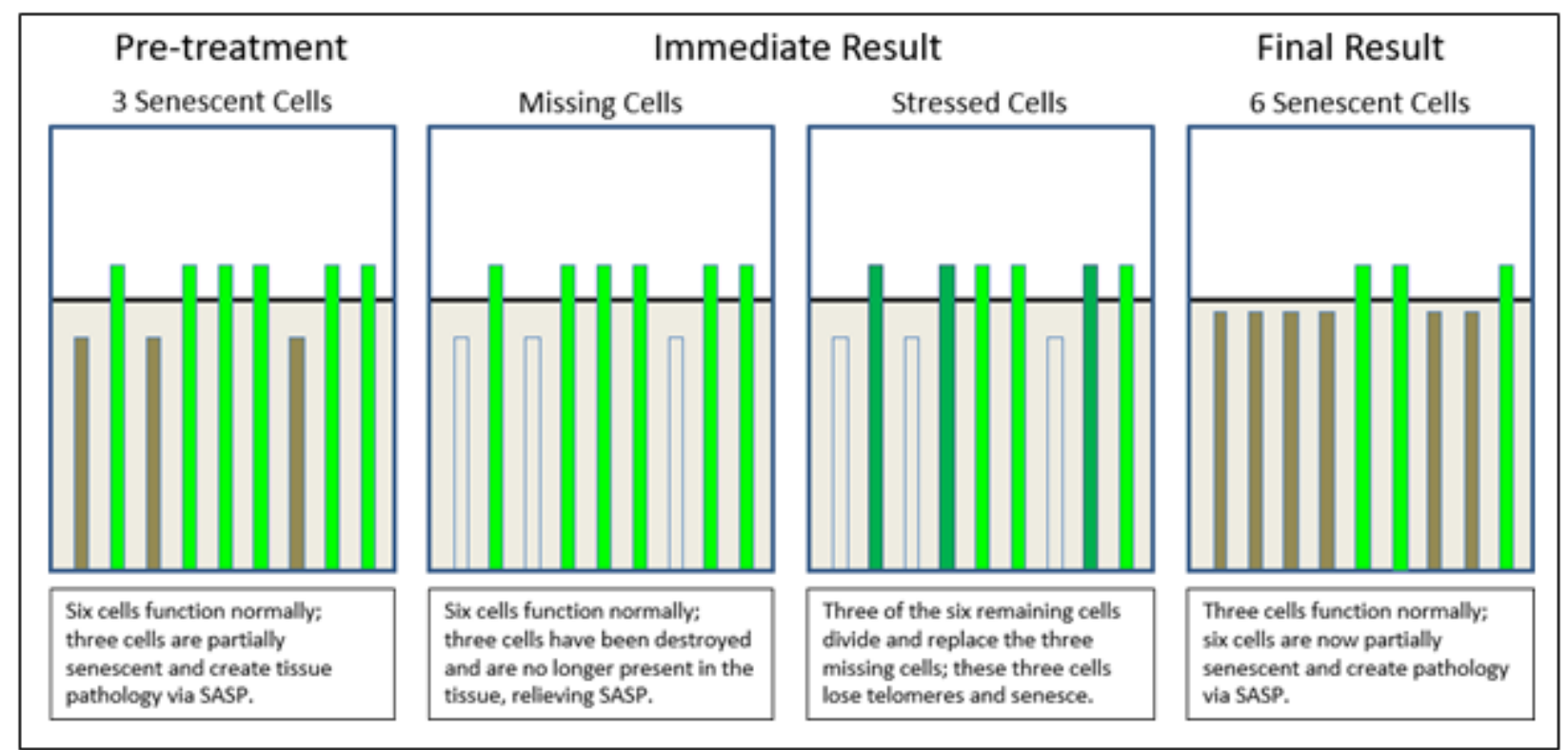

Figure 2 Senolytic effects on telomere lengths and senescence in treated tissue.

In Figure 3, we see a second graphic example in which we look down on a joint surface as composed of chondrocytes. In the pre-treatment phase, we see ten initially senescent cells are creating tissue pathology via SASP. In the intermediate phase, we have destroyed the ten senescent cells, but thereby triggered cell division in neighboring cells, whose telomeres then shorten in consequence. The final result is twenty senescent cells and an overall increase in tissue pathology via SASP. Again, we have accelerated the very tissue pathology that we intended to treat. 


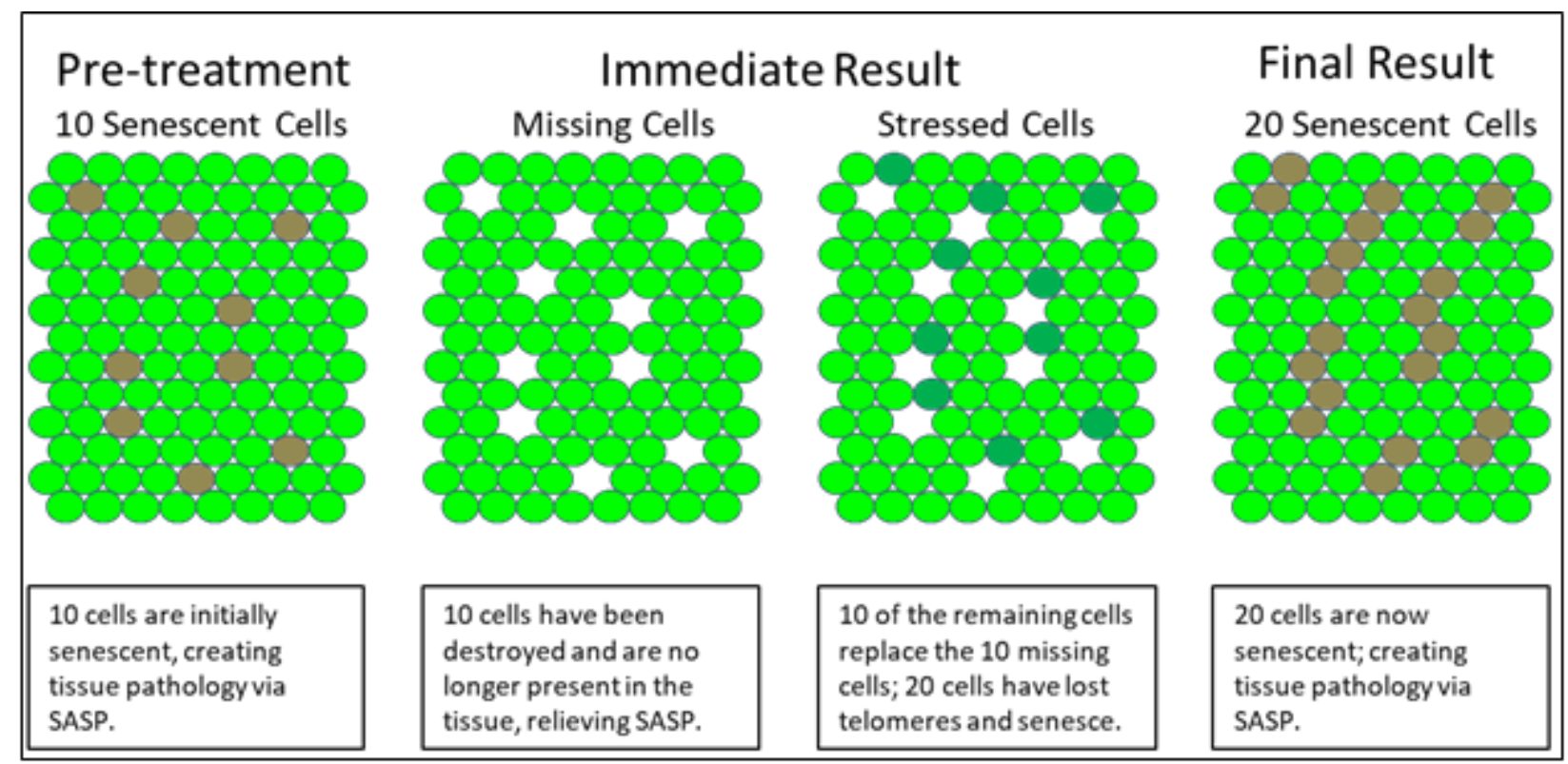

Figure 3 Senolytic effects on tissue senescence.

Published data (see below) is in line with the effects predicted above. Specifically, there is an initial improvement as measured by a number of bio-markers, followed by an accelerated decline in the same bio-markers. For example, in the curves shown in Figure 4, the purple curves represent mortality in untreated mice. The red curves represent mortality in mice treated with senolytics. The above theoretical considerations predict that mortality (as well as pathology) in treated animals should first show a transient improvement, which is seen in the initial flattening of the curves (here labelled as "Initial decreased mortality", followed by a predicted acceleration of mortality (as well as pathology) in the treated animals (here labelled as "increased mortality"). These curves represent published data on senolytic therapy trials in animals [27] and should prompt concern for the outcomes in human clinical trials.

Additional adverse effects may also ensue for several other reasons. One possibility that has been raised is "the release of proinflammatory, danger-associated molecular patterns, futher exacerbating systemic chronic inflammation" [36]. Systemic side effects may also include thrombocytopenia and immune suppression [50], as well as preventing the potential roles of senescent cells in increasing stem cell differentiation, promotion of wound healing, etc. [51].

Current publications on the use of senolytic therapies suggest (short-term) benefits, but not extended benefits when followed over time, nor address long-term risks. In addition, the published data concentrates on prevention, rather than upon the effects in older organisms with established pathology, the more likely scenario in human patients. Despite these and other limitations, published studies tout senolytic therapy $[28,50,52]$ for their clinical potential. Such studies have multiple limitations, however, including:

1) Genetically altered animals may not adequately represent the pathology found in aging human patients $[16,53]$. Such models frequently genetically-modify an animal (for example, to increase tau tangle production) then show that senolytics may ameliorate the artificially elevated bio-marker. Inferences to human pathology from such models are tenuous and may be both unsupported and unwarranted. 
2) Studies often examine animals only through "middle age" rather than into advanced age. For example, they may follow mice to less than mid-lifespan and not into old age. As the lifespan of mice is typically 24-36 months, studies that purport to demonstrate value by following mice to only 6-8 months [16] (similar to a human of 20 years of age?) cannot adequately demonstrate effects in aging mice, let alone aging humans.

3) When animals are treated with senolytics at an appropriately advanced age, they are generally followed for only short periods, or the long-term acceleration in pathology is not mentioned in the analysis, conclusions, or discussion of the data [27].

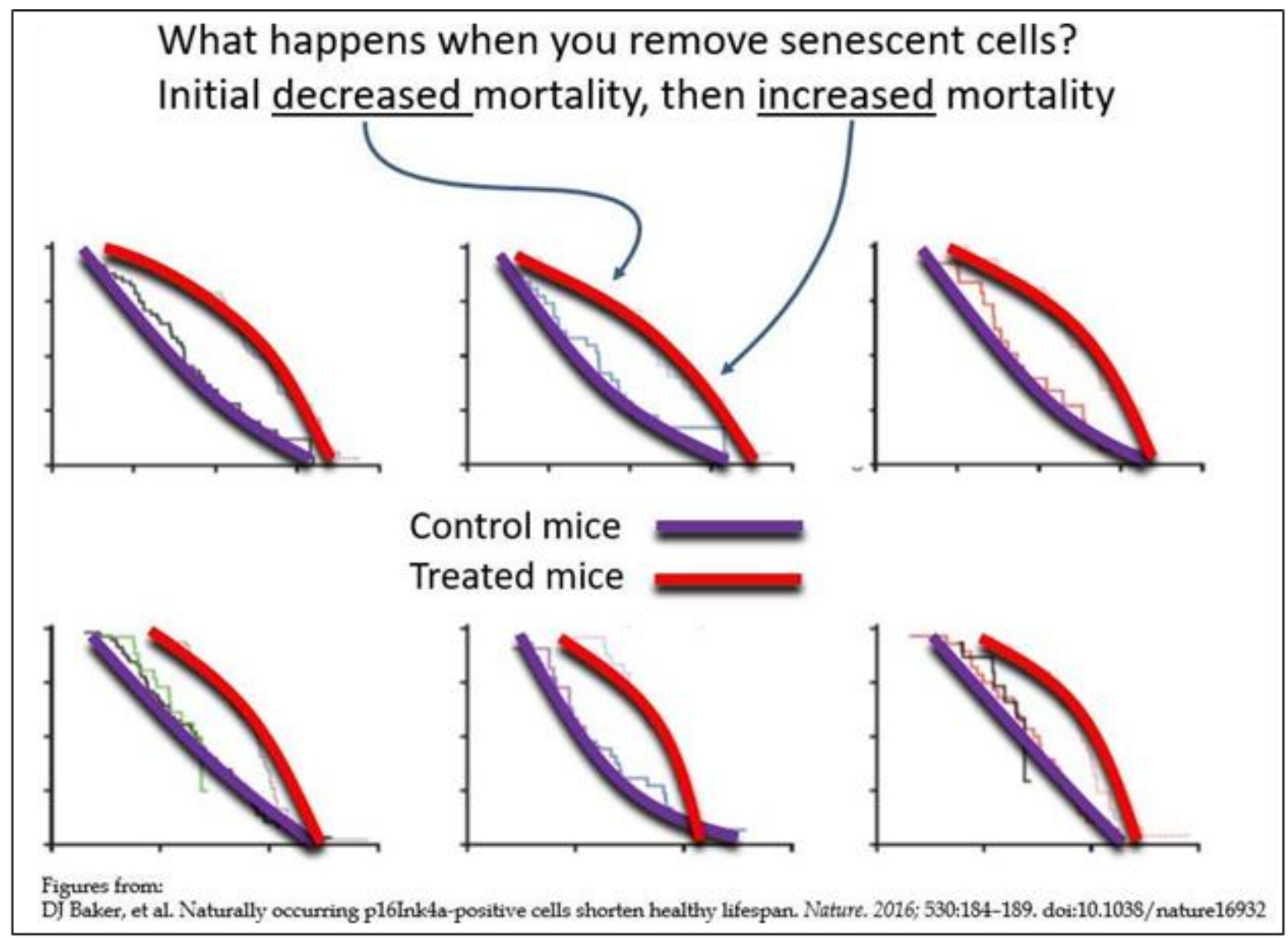

Figure 4 Senolytic agents and their outcomes.

\section{Implications and Summary}

Despite the lack of data on long-term human benefit or efficacy, considerable investments have been made in senolytic therapy $[47,54]$. The financial and the clinical risks remain underestimated. The risks are not merely those expected of any novel therapeutic agent, but are predictable, substantial, and due to naive assumptions in regard to human physiology and age- related pathology in the light of in vivo cell senescence. The acknowledged risks are routine and predictable in biotechnology: senolytics have yet to be tested in humans, there is uncertainty regarding mechanisms, and some agents are ineffective in clearing senescent cells. However, there are additional unacknowledged risks and these are substantial: senolytics will accelerate 
tissue pathology, exacerbate clinical disease, and cause early morbidity and mortality. Senolytic therapy will be clinically counter-productive.

The media $[17,52,55,56]$ and companies involved in this approach remain optimistic, but the animal data suggests that any initial clinical improvement will be followed by increased pathology and an accelerated disease course. In human trials [37], we should expect acceleration of clinical disease as the outcome. Succinctly, we should rationally predict that the long-term clinical outcome with senolytics would be worse than would occur without senolytics.

Looked at in terms of the clinical lifespan of a normally aging tissue (e.g., the joint surface of a human knee as it ages and osteoarthritis ensues), we would expect function of an untreated tissue in question to decline linearly, barring clinical intervention. This normative clinical outcome can be represented (see Figure 5) as "Normal Tissue Aging" (in purple). Repetitive injury (e.g., repetitive trauma to a knee joint in the case of a basketball player, hypertension with accelerated rheological trauma in the aorta, or traumatic brain injury in the case of the CNS) can be represented as "Repetitive Injury" (in yellow). Senolytic intervention should cause an initial improvement in clinical status followed by an accelerated clinical failure with the steepening curve, represented here as "Delete Senescent Cells" (in red). This is precisely what we see in published senolytic data [27]. Finally, telomerase therapy should cause improvement at both the cell [8] and the tissue levels (as it does at organ and biomarker level $[18,19])$ represented here as "Reset Senescent Cells" (in green).

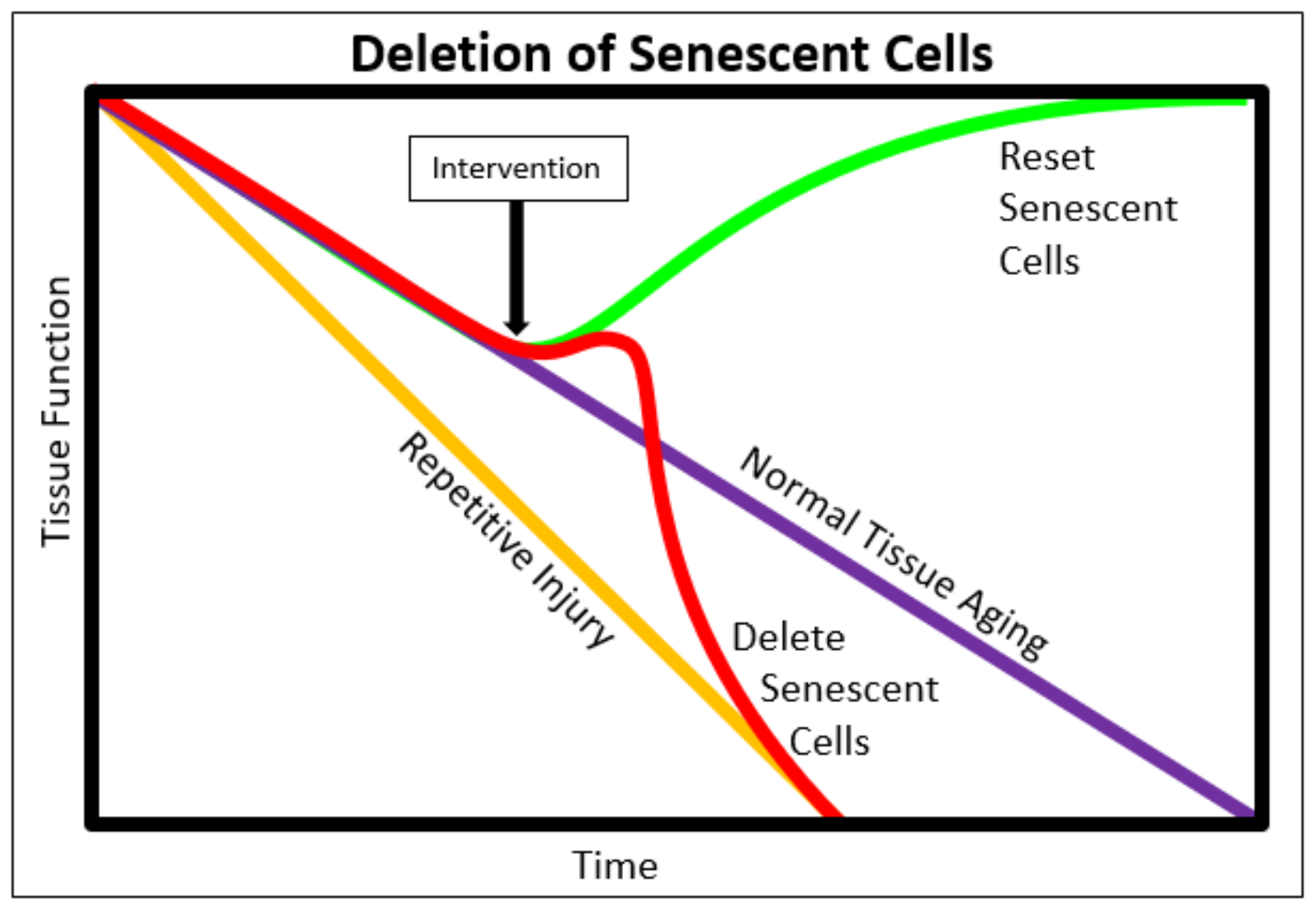

Figure 5 Comparison of senolytic agents, telomerase, repetitive injury, and controls. 
As seen in Figure 5, the expected outcome of senolytic agents ("delete senescent cells") contrasts markedly with the course of either untreated tissue ("normal tissue aging") or telomerase therapy ("reset senescent cells"). Telomerase gene therapy does not accelerate senescence in the remaining cells, and results in improved tissue function and resolution of agerelated disease. In contrast, senolytic agents accelerate senescence in remaining cells, and, in the long-term, would achieve the same outcome as repetitive tissue injury, i.e., accelerated tissue aging and increased clinical pathology.

The data curves of senolytic therapy display rectangularization of the disease course: the curve moves forward, followed by a more rapid acceleration of pathology. In a sense, this is a parallel to historical approaches to treating age-related disease, in which we see a rectangularization of lifespan, as we delay symptoms but do not affect the fundamental pathologic processes that underlie age-related disease.

The major risk of senolytic therapy lies not in its short-term efficacy, but in its long-term, predictably negative consequences. The short-term implications are potentially desirable; the long-term implications - as supported by a more thorough understanding of complex tissue pathology of age-related disease and by such long-term data as is available - are likely to be detrimental both to the age-related diseases for which senolytics are intended and to the lifespan of the elderly patients to whom senolytics are clinically relevant. Senolytic interventions overlook the physiology and the role of cell senescence in age-related clinical pathology and are likely to prove clinically catastrophic.

\section{Author Contributions}

The author wrote and edited the paper.

\section{Competing Interests}

The author is the founder and president of Telocyte LLC.

\section{References}

1. Hayflick L, Moorhead PS. The serial cultivation of human diploid strains. Exp Cell Res. 1961; 25: 585-621.

2. Hayflick L. The limited in vitro lifetime of human diploid cell strains. Exp Cell Res. 1965; 37 : 614-636.

3. Harley $\mathrm{CB}$, Futcher $\mathrm{AB}$, Greider CW. Telomeres shorten during aging of human fibroblasts. Nature. 1990; 345: 458-460.

4. Allsopp RC, Vaziri H, Patterson C, Goldstein S, Younglai EV, Futcher AB, et al. Telomere length predicts replicative capacity of human fibroblasts. Proc Natl Acad Sci U S A. 1992; 89: 1011410118.

5. Allsopp RC, Chang E, Kashefi Aazam M, Rogaev El, Piatyszek MA, Shay JW, et al. Telomere shortening is associated with cell division in vitro and in vivo. Exp Cell Res. 1995; 220: 194-200.

6. Shelton DN, Chang E, Whittier PS, Choi D, Funk WD. Microarray analysis of replicative senescence. Curr Biol. 1999; 9: 939-945. 
7. Funk WD, Wang CK, Shelton DN, Harley CB, Pagon GD, Hoeffler WK. Telomerase expression restores dermal integrity to in vitro-aged fibroblasts in a reconstituted skin model. Exp Cell Res. 2000; 258: 270-278.

8. Bodnar AG, Chiu C, Frolkis M, Harley CB, Holt SE, Lichtsteiner S, et al. Extension of life-span by introduction of telomerase into normal human cells. Science. 1998; 279: 349-352.

9. Banks DA, Fossel M. Telomeres, cancer, and aging; Altering the human lifespan. JAMA. 1997; 278: 1345-1348.

10. Fossel M. Telomerase and the aging cell; Implications for human health. JAMA. 1998; 279 : 1732-1735.

11. Fossel M. Cells, aging, and human disease. New York: Oxford University Press; 2004.

12. Chang E, Harley CB. Telomere length and replicative aging in human vascular tissues. Proc Nat Acad Sci USA. 1995; 92: 11190-11194.

13. Flanary BE, Sammons NW, Nguyen C, Walker D, Streit WJ. Evidence that aging and amyloid promote microglial cell senescence. Rejuv Res. 2007; 10: 61-74.

14. Boccardi V, Pelini L, Ercolani S, Ruggiero C, Mecocci P. From cellular senescence to Alzheimer's disease: The role of telomere shortening. Ageing Re Rev. 2015; 22: 1-8.

15. Hickman SE, Allison EK, Khoury JE. Microglial dysfunction and defective amyloid clearance pathways in aging alzheimer's disease mice. J Neurosci. 2008; 28; 8354-8360.

16. Bussian TJ, Aziz A, Meyer CF, Swenson BL, van Deursen JM, Baker DJ. Clearance of senescent glial cells prevents tau-dependent pathology and cognitive decline. Nature. 2018; 562: 578582.

17. Penney J, Tsai LH. Elimination of senescent cells prevents neurodegeneration in mice. Nature. 2018; 562: 503-504.

18. Jaskelioff M, Muller FL, Paik JH, Thomas E, Jiang S, Adams A, et al. Telomerase reactivation reverses tissue degeneration in aged telomerase deficient mice. Nature. 2011; 469: 102-106.

19. de Jesus BB, Vera E, Schneeberger K, Tejera AM, Ayuso E, Bosch F, et al. Telomerase gene therapy in adult and old mice delays aging and increases longevity without increasing cancer. EMBO Mol Med. 2012; 4: 691-704.

20. Herbig U, Ferreira M, Condel L, Carey D, Sedivy JM. Cellular senescence in aging primates. Science. 2006; 311: 1257.

21. Coppé JP, Desprez PY, Krtolica A, Campisi J. The senescence-associated secretory phenotype: the dark side of tumor suppression. Annu Rev Pathol. 2010; 5: 99-118.

22. Coppé JP, Patil CK, Rodier F, Sun Y, Muñoz DP, Goldstein J, et al. Senescence-associated secretory phenotypes reveal cell-nonautonomous functions of oncogenic ras and the p53 tumor suppressor. PLOS. 2008; 6: 2853-2868.

23. Childs BG, Baker DJ, Wijshake T, Conover CA, Campisi J, van Deursen JM. Senescent intimal foam cells are deleterious at all stages of atherosclerosis. Science. 2016; 354: 472-477.

24. Coppe JP, Pati CK, Rodier F, Krtolica A, Beausejour CM, Parrinello S, et al. A human- like senescence-associated secretory phenotype is conserved in mouse cells dependent on physiological oxygen. PLoS One. 2010; 5: e9188.

25. Hernandez-Segura A, de Jong TV, Melov S, Guryev V, Campisi J, Demaria M. Unmasking transcriptional heterogeneity in senescent cells. Curr Biol. 2017; 27: 2652-2660.

26. Freund A, Laberge R-M, Demaria M, Campisi J. Lamin B1 loss is a senescence-associated biomarker. Magin TM, ed. Mol Biol Cell. 2012; 23: 2066-2075. 
27. Baker DJ, Childs BG, Durik M, Wijers ME, Sieben CJ, Zhong J, et al. Naturally occurring p16Ink4a- positive cells shorten healthy lifespan. Nature. 2016; 530: 184-189.

28. Kirkland JL, Tchkonia T, Zhu Y, Niedernhofer $U$, Robbins PD. The clinical potential of senolytic drugs. J Am Geriatr Soc. 2017; 65: 2297-2301.

29. Baker DJ, Wijshake T, Tchkonia T, LeBrasseur NK, Childs BG, van de Sluis B, et al. Clearance of p16Ink4a-positive senescent cells delays ageing-associated disorders. Nature. 2011; 479: 232236.

30. These currently include: Unity Biotechnology, Cleara biotech, Senolytic Therapeutics, Oisin Biotechnologies.

31. Chang J, Wang Y, Shao L, Laberge RM, Demaria M, Campisi J, et al. Clearance of senescent cells by ABT263 rejuvenates aged hematopoietic stem cells in mice. Nat Med. 2016; 22: 78-83.

32. Grezella C, Fernandez-Rebollo E, Franzen J, Ventura Ferreira MS, Beier F, Wagner W. Effects of senolytic drugs on human mesenchymal stromal cells. Stem Cell Res Ther. 2018; 9: 108.

33. Baar MP, Brandt RMC, Putavet DA, Klein JDD, Derks KWJ, Bourgeois BRM, et al. Targeted apoptosis of senescent cells restores tissue homeostasis in response to chemotoxicity and aging. Cell. 2017; 169: 132-147.

34. Both, as of 2018, from Unity Biotechnology.

35. Zhu Y, Tchkonia T, Pirtskhalava T, Gower AC, Ding H, Giorgadze N, et al. The Achilles' heel of senescent cells: from transcriptome to senolytic drugs. Aging Cell. 2015; 14: 644-658.

36. McHugh D, Gil J. Senescence and aging: Causes, consequences, and therapeutic avenues. J Cell Biol. 2018; 217: 65-77.

37. Tchkonia T, Kirkland JL. Aging, cell senescence, and chronic disease - emerging therapeutic strategies. JAMA. 2018; 320: 1319-1320.

38. Jeon $\mathrm{OH}$, Kim C, Laberge RM, Demaria $M$, Rathod S, Vasserot AP, et al. Local clearance of senescent cells attenuates the development of post-traumatic osteoarthritis and creates a pro- regenerative environment. Nat Med. 2017; 23: 775-781.

39. Demaria M, O'Leary MN, Chang J, Shao L, Liu S, Alimirah F, et al. Cellular senescence promotes adverse effects of chemotherapy and cancer relapse. Cancer Discov. 2017; 7: 165-176.

40. Baker DJ, Petersen RC. Cellular senescence in brain aging and neurodegenerative diseases: evidence and perspectives. J Clin Invest. 2018; 128: 1208-1216.

41. Kumazaki T. Modulation of gene expression during aging of human vascular endothelial cells. Hiroshima J Med Sci. 1993; 42: 97.

42. Cooper LT, Cooke JP, Dzau VJ. The vasculopathy of aging. J Gerontol. 1994; 49: 191-196.

43. Yang $Y$, Wilson DL. Characterization of a life-extending mutation in age-2, a new aging gene in Caenorhabditis elegans. J Gerontol A Biol Sci Med Sci. 1999; 54: B137-B142.

44. Fossel M. Reversing human aging. New York: William Morrow and Company; 1996.

45. Fossel M. Implications of recent work in telomeres and cell senescence. J Anti-Aging Med. 1998; 1: 39-43.

46. Fossel M. Cell senescence and human aging: A review of the theory. In Vivo. 2000; 14: 29-34.

47. https://www.fiercebiotech.com/biotech/unity-files-for-85m-ipo-to-take-anti-aging-drugsinto-phase-

1?mkt_tok=eyJpljoiTUdJNVpUQXIZMIE1TIRObCIsInQiOiJISjNzRER4Zks1TzJTRTJ6XC9SaEVSRWJ DNTMzZDFsU2NvbOV2bHQzM2syRWdKZW1VVTJaV0xNRkFZaOJaMEZvZG43SkdNQXAzM1Bob 


\section{HIIQURhOVhrSjJJUnFGcjIFSmQ4QWpTMGNIR2dONHhXV1RTUG5UMzkxTFBHRVhjY1JFWDMif Q\%3D\%3D\&mrkid $=20453317$}

48. van Deursen JM. The role of senesent cells in ageing. Nature. 2014; 509: 439-446.

49. Shao L, Feng W, Li H, Gardner D, Luo Y, Wang Y, et al. Total body irradiation causes long-term mouse BM injury via induction of HSC premature senescence in an Ink4a- and Arfindependent manner. Blood. 2014; 123: 3105-3115.

50. Childs BG, Gluscevic M, Baker DJ, Laberge RM, Marquess D, Dananberg J, et al. Senescent cells: an emerging target for diseases of ageing. Nat Rev Drug Discov. 2017; 16: 718-735.

51. Demaria M, Ohtani N, Youssef SA, Rodier F, Toussaint W, Mitchell JR, et al. An essential role for senescent cells in optimal wound healing through secretion of PDGF-AA. Dev Cell. 2014; 31: 722-733.

52. https://www.alzforum.org/news/research-news/are-tauopathies-caused-neuronal-and-glialsenescence

53. Musi N, Valentine JM, Sickora KR, Baeuerle E, Thompson CS, Shen Q, et al. Tau protein aggregation is associated with cellular senescence in the brain. Aging Cell. 2018; 17: e12840.

54. https://www.fiercebiotech.com/biotech/anti-aging-outfit-unity-bags-55m-and-looks-to-clinic

55. https://newatlas.com/fisetin-flavonoid-anti-aging-mouse-study/56610/

56. https://www.technologyreview.com/s/612284/finally-the-drug-that-keeps-you-young/

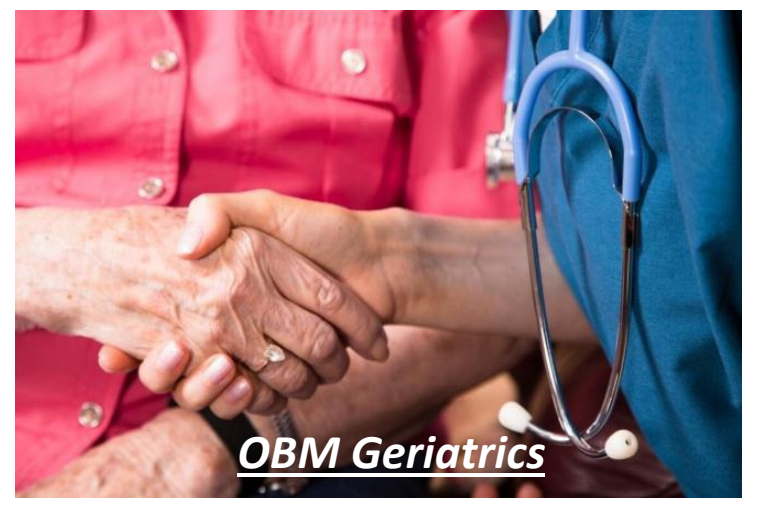

Enjoy OBM Geriatrics by:

1. Submitting a manuscript

2. Joining in volunteer reviewer bank

3. Joining Editorial Board

4. Guest editing a special issue

For more details, please visit:

http://www.lidsen.com/journals/geriatrics 


\title{
Effect of Six-Months Supplementation with Cholecalciferol on Glycemic and Blood Pressure Control in Elderly Type 2 Diabetic Patients with Vitamin D Deficiency: A Pilot Study
}

\author{
Marco Barale *, Ruth Rossetto Giaccherino, Ezio Ghigo, Massimo Procopio
}

Division of Endocrinology, Diabetology and Metabolic Diseases, Department of General and Specialty Medicine, Molinette Hospital, University of Turin - CsoDogliotti, 14 - 10126 Turin, Italy; EMails:_marco.barale@unito.it; ruth.rossetto@unito.it; ezio.ghigo@unito.it; massimo.procopio@unito.it

* Correspondence: Marco Barale; E-Mail: marco.barale@unito.it

Academic Editor: Michael Fossel

\section{OBM Geriatrics}

2019, volume3,issue 1

doi:10.21926/obm.geriatr.1901033
Received: October 31, 2018

Accepted: January 29, 2019

Published: February 14, 2019

\begin{abstract}
Background: It is well known that hypovitaminosis $D$ has been associated with various cardio-metabolic disorders, though the pathogenetic link, if any, still remains unclear. Our aim was to evaluate in elderly uncontrolled non-insulin-treated type 2 diabetic patients with hypovitaminosis $D$, whether six-months vitamin $D$ supplementation was able to improve glycemic control, lipid profile and blood pressure levels.

Methods: In an open-label pilot study, thirty type 2 diabetic patients (age $71.5 \pm 3.2$ years, BMI $\left.29.7 \pm 3.5 \mathrm{~kg} / \mathrm{m}^{2}\right)$ with hypovitaminosis D (25OHvitamin D $\left.22.02 \pm 11.31 \mathrm{nmol} / \mathrm{l}\right)$ were randomized to cholecalciferol supplementation $(500 \mathrm{UI} / \mathrm{kg}$ p. o. weekly, +D) or no intervention (-D) for six months. At baseline, three and six months all patients were evaluated for serum glucose, glycosylated hemoglobin (HbA1c), lipid profile, systolic (SBP) and diastolic (DBP) blood pressure levels, while calcium mineral metabolism parameters were assessed in all patients at baseline and after vitamin D supplementation.

Results: Vitamin D supplementation had a beneficial effect on fasting glucose (mean percentage changes $\pm S D,-11.0 \% \pm 19.1$ vs $+7.4 \% \pm 19.7$, respectively; $p<0.04)$ and SBP
\end{abstract}

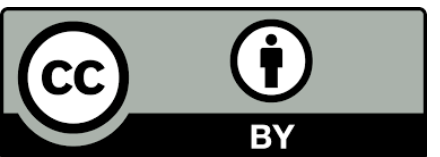

(C)2019 by the author. This is an open access article distributed under the conditions of the Creative Commons by Attribution License, which permits unrestricted use, distribution, and reproduction in any mediumor format, provided the original work is correctly cited. 
levels $(-4.7 \% \pm 9.0$ vs $+2.9 \% \pm 12.1$, respectively; $p<0.05)$ and led to favorable but not significant changes in serum $\mathrm{HbA1C}$ and $\mathrm{DBP}$ levels in $+\mathrm{D}$ vs $-\mathrm{D}$ patients. In the former, fasting glucose $(8.15 \pm 1.57$ vs $9.36 \pm 1.83 \mathrm{mmol} / \mathrm{l})$, HBA1c $(63.4 \pm 11.6$ vs $74.8 \pm 13.8$ $\mathrm{mmol} / \mathrm{mol})$, SBP $(149.7 \pm 16.2$ vs $157.5 \pm 14.7 \mathrm{mmHg})$ and DBP $(79.1 \pm 6.4$ vs $83.4 \pm 4.4$ $\mathrm{mmHg}$ ) levels improved at six months vs baseline, respectively ( $<<0.05$ for all comparisons). After six-months supplementation, we noticed an inverse relationship of serum 250 Hvitamin D levels with HbA1c $(R=-0.66, p<0.01)$.

Conclusions: Our data in elderly patients with poor controlled type 2 diabetes mellitus and vitamin $D$ deficiency indicate that an adequate six-months supplementation of cholecalciferol, able to restore normal 250 Hvitamin D levels, can significantly improve fasting glucose and SBP levels.

\section{Keywords}

Vitamin D deficiency; cholecalciferol; type 2 diabetes mellitus; hypertension; dyslipidemia

\section{Introduction}

It is well known that hypovitaminosis $D$ has been associated with various cardio-metabolic disorders, though the pathogenetic link, if any, still remains unclear. Many pre-clinical and animal studies have demonstrated that various tissues involved in the regulation of glucose and lipid metabolism, as well as in blood pressure control, possess specific vitamin D receptors and enzymes able to locally synthesize calcitriol, accounting for paracrine effects of vitamin D on these systemic functions and the association between hypovitaminosis $D$ and cardio-metabolic alterations [1].

Accordingly, many epidemiological studies have shown a correlation between low serum 250 Hvitamin D (25OHD) levels and increased prevalence of type 2 diabetes mellitus (T2DM), hypertension, dyslipidemia and metabolic syndrome [2]. Moreover, in T2DM lower serum 25OHD levels and increased frequency of hypovitaminosis $D$ have been reported $[3,4]$ and some data show an association of vitamin D deficiency with enhanced cardiovascular mortality [5]. However, randomized-controlled trials, systematic reviews and meta-analysis have reported inconsistent results on beneficial effects of vitamin D supplementation on cardio-metabolic outcomes. This is probably due to a wide heterogeneity of studied patients' characteristics (i.e. age, body weight, metabolic and blood pressure control, vitamin D status) and vitamin D supplementation (form, route, dose, frequency and duration of administration) [6-9].

Thus, the aim of our pilot study was to evaluate in uncontrolled non-insulin-treated type 2 diabetic patients with hypovitaminosis $D$, whether six-months vitamin $D$ supplementation was able to improve glycemic control, lipid profile and blood pressure levels.

\section{Materials and Methods}

Among subjects referred consecutively to our Diabetes Care Centre, thirty patients with 1) known T2DM, 2) aged 50-80 years, 3) with BMI 25-39 kg/m², 4) on treatment with anti-diabetic drugs (sulphonylureas, glinides, metformin, thiazolidinediones, dipeptidyl peptidase-4 inhibitors, 
glucagon-like peptide 1 receptor agonists, sodium glucose co-transporter 2 inhibitors), 5) with poor glycemic control (HBA1c $>59 \mathrm{mmol} / \mathrm{mol}$ ) since at least three months before enrollment and 6) hypovitaminosis D $(25 \mathrm{OHD}<75 \mathrm{nmol} / \mathrm{l})$ were recruited to perform an open-label randomized controlled pilot study. Exclusion criteria included 1) fertile women, 2) patients with metabolic bone diseases (Paget's disease of bone, primary hyperparathyroidism, idiopathic hypercalciuria), 3) cancer diseases, 4) liver, renal, heart or respiratory failure, 5) intestinal malabsorption and 6) patients on treatment with insulin 7) patients with a recent history of anti-diabetic medication changes during the three months prior to the enrollment 8) patients with drugs known to affect bone mineral metabolism (i.e. barbiturates, corticosteroids, anti-osteoporotic agents, calcium and vitamin D supplements).

This study was performed according to the Declaration of Helsinki II and approved by the local Ethics Committee.

Project identification code: 0050172

Date of approval: 29/04/2013

Name of the ethics committee: Comitato Etico Interaziendale

AOU S. Giovanni Battista di Torino / AO CTO / Maria Adelaide di Torino.

Eligible participants who signed informed consent were assigned into two groups according to a computer-generated random numbers and followed up for six months.

As to administered vitamin D dosage, in order to obtain extra-skeletal effects, we considered a sufficient 25OHD target level of 75-125 nmol/l and an optimal 25OHD target level of 125-175 $\mathrm{nmol} / \mathrm{l}[10,11]$. Moreover, we hypothesized the presence of a severe deficiency status in our patients (mean $25 \mathrm{OHD}$ levels $<25 \mathrm{nmol} / \mathrm{l}$ ) and we estimated an increase in $25 \mathrm{OHD}$ levels of about $2.5 \mathrm{nmol} / \mathrm{I}$ for each $100 \mathrm{UI} / \mathrm{die}(2.5 \mathrm{mcg} / \mathrm{die})$ of given cholecalciferol [12]. Then, we calculated the weekly dosage to administer in a normal-weight patient according to the following formula: [(mean optimal $250 \mathrm{HD}$ levels - mean baseline levels)*100 UI]*7 days/70 kg. The same dosage was maintained for each patient throughout the study.

After randomization, sixteen patients were supplemented with $500 \mathrm{UI} / \mathrm{kg}$ oral cholecalciferol once a week, while fourteen patients were observed as control group without intervention for six months. Subjects supplemented returned vitamin D bottles to assess treatment compliance. All patients were not allowed to take other vitamin D supplements.

Anti-diabetic and anti-hypertensive therapy might have been changed in order to improve disease control according to current guidelines.

At baseline, three and six months all patients were evaluated for serum glucose, glycosylated hemoglobin ( $\mathrm{HbA1c}$ ), lipid, SBP, DBP and creatinine levels, while calcium mineral metabolism parameters (both serum and urinary calcium and phosphate, serum PTH, 25OHD, total alkaline phosphatase (ALP) levels) were assessed in all patients at baseline and after six-months vitamin D supplementation.

Moreover, at each participant's visit, we collected weight and height, we calculated BMI and we measured SBP and DBP. The average of three measurements in a seated position after a 5 minute rest was considered as the final SBP and DBP. Calcium dietary intake was estimated based on a selfreported questionnaire; patients who did not achieve recommended dietary requirements for calcium [13] were treated with oral calcium supplements (500 mg/die of calcium carbonate).

Diagnosis of dyslipidemia and hypertension were based on medical history and according to WHO NCEP ATP III criteria [14] and The Task Force for the Management of Arterial Hypertension of 
the European Society of Hypertension and the European Society of Cardiology [15], respectively. In line with Endocrine Society Guidelines, vitamin D insufficiency, deficiency and severe deficiency were defined as serum 250HD between 50 and 74, between 25 and 49 and below $25 \mathrm{nmol} / \mathrm{l}$, respectively [16].

Plasma glucose $(\mathrm{mmol} / \mathrm{l})$, serum total $(\mathrm{mmol} / \mathrm{l})$ and $\mathrm{HDL}$ cholesterol $(\mathrm{mmol} / \mathrm{l})$, triglycerides $(\mathrm{mmol} / \mathrm{l})$ and creatinine $(\mu \mathrm{mol} / \mathrm{l})$ levels were measured by enzymatic colorimetric tests (Cobas, Roche). LDL cholesterol was calculated using Friedewald's formula. HbA1c levels were measured through high performance liquid chromatography (HPLC). Glomerular filtration rate (GFR) was calculated according to Cockroft-Gault formula.

Serum and urinary calcium and phosphate ( $\mathrm{mmol} / \mathrm{l}$ and $\mathrm{mmol} / \mathrm{die}$ ) were tested using automated methods based on colorimetric and enzymatic assays (Cobas, Roche). Serum intact parathormone (PTH) assay (pmol/l) based on an immunoradiometric sandwich method (IRMA) that used two polyclonal antibodies (DiaSorin): an antibody recognizing the C-terminal region (aa 39-84) was used as the capture antibody while an antibody recognizing the $\mathrm{N}$-terminal region was used for detection; inter- and intra-assay coefficient of variation were $5.5 \%$ and below $3 \%$, respectively. Serum $250 H D$ ( $\mathrm{nmol} / \mathrm{l}$ ) was tested by a radioimmunoassay method using an antibody with specificity to 25OHD (DiaSorin). ALP (UI/I) was tested using colorimetric assay in accordance with a standardized method (Cobas, Roche): in the presence of magnesium and zinc ions, $p$ nitrophenyl phosphate was cleaved by phosphatases into phosphate and p-nitrophenol, proportional to the ALP activity that was measured photometrically. Serum bone alkaline phosphatase (BAP, $\mu \mathrm{g} / \mathrm{l}$ ) was measured by an immunoradiometric sandwich method that use mouse monoclonal antibodies directed against two different epitopes of BAP and hence not competing (Beckman Coulter).

Data are presented as mean \pm SD or as mean, 95\% confidence interval (CI). Normality of frequency distribution functions was tested by the Shapiro-Wilk W-test. Significant differences were sought by the Mann-Whitney $U$ test. Spearman's $R$ coefficient was used to look for associations of calcium metabolism parameters with glucose, $\mathrm{HbA} 1 \mathrm{c}$, lipid profile and SBP and DBP levels. Calculations were performed using SPSS Windows release 24.0; $p<0.05$ was considered significant.

\section{Results}

\subsection{Baseline}

Clinical and biochemical features of enrolled patients (21 men and 9 women) both as a whole and separately as a group undergoing either vitamin $D(+D)$ or no vitamin $D(-D)$ supplementation are shown in Table 1 and 2. As reported, at baseline, we have found no differences among the two groups in clinical, anthropometric and biochemical parameters, except for higher serum glucose and lower phosphate levels in patients randomized to $+D$.

At the time of enrollment, all patients were on anti-diabetic drugs, specifically (frequency, 95\% $\mathrm{Cl}$ ): sulphonylureas 63.3\%, 46.1-80.6, glinides 16.7\%, 3.3-30.0, metformin 83.3\%, 70.0-96.7, thiazolidinediones $26.7 \%, 10.8-42.5$, acarbose $6.7 \%$, 0-15.6, dipeptidyl peptidase-4 inhibitors 53.3\%, 35.5-71.2, glucagon-like peptide 1 receptor agonists 10\%, 0-20.7. 
Table 1 Age, BMI, blood pressure levels, parameters of glucose and lipid metabolism and renal function in type 2 diabetic patients both as a whole and separately as a group undergoing either vitamin $D(+D)$ or no vitamin $D(-D)$ supplementation.

\begin{tabular}{|c|c|c|c|c|}
\hline & $\begin{array}{l}\text { Whole group } \\
(n=30)\end{array}$ & $\begin{array}{l}\text { +D patients } \\
(\mathrm{n}=16)\end{array}$ & $\begin{array}{l}\text {-D patients } \\
(n=14)\end{array}$ & \begin{tabular}{|l} 
Reference \\
range
\end{tabular} \\
\hline Age (years) & $71.5 \pm 3.2$ & $71.6 \pm 3.5$ & $71.4 \pm 3.1$ & \\
\hline $\mathrm{BMI}\left(\mathrm{kg} / \mathrm{m}^{2}\right)$ & $29.7 \pm 3.5$ & $29.2 \pm 3.8$ & $30.3 \pm 3.2$ & $18.5-24.9$ \\
\hline Systolic blood pressure (mmHg) & $153.3 \pm 17.8$ & $157.5 \pm 14.7$ & $148.6 \pm 20.2$ & $90-140$ \\
\hline Diastolic blood pressure $(\mathrm{mmHg})$ & $83.7 \pm 7.3$ & $83.4 \pm 4.4$ & $83.9 \pm 9.8$ & $60-90$ \\
\hline Glucose $(\mathrm{mmol} / \mathrm{l})$ & $8.6 \pm 1.81$ & $9.36 \pm 1.83^{*}$ & $7.73 \pm 1.38$ & $4.0-5.9$ \\
\hline Glycosylated Hemoglobin (mmol/mol) & $70.8 \pm 11.4$ & $74.8 \pm 13.8$ & $66.3 \pm 5.2$ & $20-38$ \\
\hline Total cholesterol (mmol/l) & $4.24 \pm 0.98$ & $4.27 \pm 0.84$ & $4.19 \pm 1.15$ & $<5.2$ \\
\hline HDL cholesterol (mmol/l) & $1.38 \pm 0.44$ & $1.30 \pm 0.30$ & $1.48 \pm 0.56$ & $\begin{array}{l}\geq 1.0(\mathrm{M}) \\
\geq 1.3(\mathrm{~F})\end{array}$ \\
\hline Triglycerides (mmol/l) & $1.40 \pm 0.66$ & $1.46 \pm 0.58$ & $1.32 \pm 0.76$ & $<1.7$ \\
\hline LDL cholesterol (mmol/l) & $2.15 \pm 0.80$ & $2.18 \pm 0.53$ & $2.12 \pm 1.08$ & $<4.1$ \\
\hline Non-HDL cholesterol(mmol/l) & $2.85 \pm 1.01$ & $2.98 \pm 0.86$ & $2.72 \pm 1.18$ & $<3.4$ \\
\hline Creatinine $(\mu \mathrm{mol} / \mathrm{l})$ & $77.23 \pm 20.02$ & $76.21 \pm 20.97$ & $78.49 \pm 19.55$ & $44.2-106.1$ \\
\hline Glomerular filtration rate $(\mathrm{ml} / \mathrm{s})$ & $1.44 \pm 0.32$ & $1.43 \pm 0.28$ & $1.46 \pm 0.37$ & $1.2-2.0$ \\
\hline
\end{tabular}

${ }^{*} \mathrm{p}<0.05$ vs $-\mathrm{D}$ patients.

Table 2 Parameters of calcium mineral metabolism in type 2 diabetic patients both as a whole and separately as a group undergoing either vitamin $D(+D)$ or no vitamin $D(-D)$ supplementation.

\begin{tabular}{|l|l|l|l|l|}
\hline & $\begin{array}{l}\text { Whole group } \\
(\mathrm{n}=30)\end{array}$ & $\begin{array}{l}+\mathrm{D} \text { patients } \\
(\mathrm{n}=16)\end{array}$ & $\begin{array}{l}-\mathrm{D} \text { patients } \\
(\mathrm{n}=14)\end{array}$ & $\begin{array}{l}\text { Reference } \\
\text { range }\end{array}$ \\
\hline Serum total calcium $(\mathrm{mmol} / \mathrm{I})$ & $2.4 \pm 0.1$ & $2.3 \pm 0.1$ & $2.4 \pm 0.1$ & $2.2-2.6$ \\
\hline Serum phosphate $(\mathrm{mmol} / \mathrm{l})$ & $1.0 \pm 0.2$ & $0.9 \pm 0.2^{*}$ & $1.1 \pm 0.1$ & $0.8-1.5$ \\
\hline Urinary calcium $(\mathrm{mmol} / \mathrm{die})$ & $3.1 \pm 2.1$ & $3.7 \pm 2.2$ & $2.3 \pm 1.7$ & $2.5-7.5$ \\
\hline Urinary phosphate $(\mathrm{mmol} / \mathrm{die})$ & $18.9 \pm 8.1$ & $21.2 \pm 7.8$ & $16.2 \pm 7.8$ & $12.9-32.3$ \\
\hline PTH (pmol/l) & $6.23 \pm 3.60$ & $6.62 \pm 3.95$ & $5.8 \pm 3.24$ & $1.6-6.9$ \\
\hline 25OHvitamin D (nmol/l) & $22.02 \pm 11.31$ & $21.80 \pm 11.90$ & $22.27 \pm 11.03$ & $75-125$ \\
\hline Total alkaline phosphatase $(\mathrm{UI} / \mathrm{l})$ & $66.5 \pm 21.1$ & $70.5 \pm 20.9$ & $62.0 \pm 21.2$ & $\begin{array}{l}53-128(\mathrm{M}) \\
42-141(\mathrm{~F})\end{array}$ \\
\hline Bone alkaline phosphatase $(\mu \mathrm{g} / \mathrm{l})$ & $10.46 \pm 4.53$ & $11.08 \pm 4.50$ & $9.75 \pm 4.63$ & $8-16$ \\
\hline
\end{tabular}

${ }^{*} \mathrm{p}<0.05$ vs $-\mathrm{D}$ patients.

Prevalence of dyslipidemia was 70\%, 53.6-86.4 in the whole group. Specifically, hypertriglyceridemia and HDL hypocholesterolemia were found in 33.3\%, 16.5-50.2, and 6.7\%, 015.6, respectively, while LDL (and/or non-HDL) cholesterol target was not achieved by 33.3\%, 16.550.2. Clinical history of hypertension was present in $56.7 \%, 38.9-74.4$, while increased blood pressure levels were registered in 90\%, 79.3-100. Use of anti-dyslipidemic and anti-hypertensive drugs were 53.3\%, 35.5-71.2 and 56.7\%, 38.9-74.4, respectively. Prevalence of overweight, first and second grade obesity were $66.7 \%, 49.8-83.5,20 \%, 5.7-34.3$ and $13.3 \%, 1.2-25.5$, respectively. 
Hypovitaminosis D was confirmed in all patients, with prevalence of insufficiency, deficiency and severe deficiency equal to $3.3 \%, 0-9.8,30 \%, 13.6-46.4$ and $66.7 \%, 49.8-83.5$, respectively. Consistently, we found increased prevalence of secondary hyperparathyroidism (30\%, 13.6-46.4).

No significant differences were registered among $+D$ and $-D$ patients in terms of gender distribution, frequency of metabolic comorbidities and related therapies.

\subsection{Calcium and Vitamin D Supplementation}

Mean dietary calcium intake was $917 \pm 252 \mathrm{mg} /$ die in the whole group, while separately in $+\mathrm{D}$ e -D was $950 \pm 258$ and $879 \pm 249 \mathrm{mg} /$ die, respectively $(p=N S)$. The supplementation of $500 \mathrm{mg}$ of calcium carbonate was administered to $8+D$ and $9-D$ patients.

After randomization, mean vitamin $D$ dosage of supplementation in $+D$ patients was weekly cholecalciferol $39359 \pm 5569 \mathrm{UI}$, with full adherence to therapy in $100 \%$ of $+\mathrm{D}$ patients.

\subsection{Three Months}

Clinical and biochemical features of $+D$ and $-D$ patients at three months are shown in Table 3. $\mathrm{BMI}$, blood pressure levels, parameters of glucose and lipid metabolism and renal function were not different among the two groups. However, compared to baseline, $+D$ patients showed a significant reduction in serum glucose $(p<0.006), \operatorname{HbA1c}(p<0.03)$ and DBP levels $(p<0.007)$, while creatinine was increased $(p<0.03)$ despite no significant variations in glomerular filtration rate. On the contrary, we registered no significant variations vs baseline in $-D$ patients, except for increased non HDL cholesterol $(p<0.03)$.

Table 3 BMI, blood pressure levels, parameters of glucose and lipid metabolismand renal function in type 2 diabetic patients with $(+D)$ and without $(-D)$ vitamin $D$ supplementation at three months.

\begin{tabular}{|c|c|c|c|}
\hline & $\begin{array}{l}+D \text { patients } \\
(n=16)\end{array}$ & $\begin{array}{l}\text {-D patients } \\
(n=14)\end{array}$ & $\begin{array}{l}\text { Reference } \\
\text { range }\end{array}$ \\
\hline $\mathrm{BMI}\left(\mathrm{kg} / \mathrm{m}^{2}\right)$ & $28.9 \pm 3.4$ & $30.1 \pm 3.5$ & $18.5-24.9$ \\
\hline Systolic blood pressure (mmHg) & $151.0 \pm 18.6$ & $147.9 \pm 19.1$ & $90-140$ \\
\hline Diastolic blood pressure (mmHg) & $76.0 \pm 9.7^{*}$ & $80.4 \pm 10.1$ & $60-90$ \\
\hline Glucose (mmol/l) & $7.84 \pm 1.38^{*}$ & $7.38 \pm 1.58$ & $4.0-5.9$ \\
\hline Glycosylated Hemoglobin $(\mathrm{mmol} / \mathrm{mol})$ & $66.5 \pm 8.5^{*}$ & $61.6 \pm 7.8$ & $20-38$ \\
\hline Total cholesterol (mmol/l) & $4.23 \pm 0.90$ & $4.39 \pm 1.15$ & $<5.2$ \\
\hline HDL cholesterol (mmol/l) & $1.25 \pm 0.36$ & $1.35 \pm 0.37$ & $\begin{array}{l}\geq 1.0(\mathrm{M}) \\
\geq 1.3(\mathrm{~F})\end{array}$ \\
\hline Triglycerides (mmol/l) & $1.63 \pm 0.93$ & $1.45 \pm 0.71$ & $<1.7$ \\
\hline LDL cholesterol $(\mathrm{mmol} / \mathrm{l})$ & $2.04 \pm 0.45$ & $2.22 \pm 0.82$ & $<4.1$ \\
\hline Non-HDL cholesterol (mmol/l) & $3.00 \pm 1.02$ & $2.83 \pm 1.03^{*}$ & $<3.4$ \\
\hline Creatinine $(\mu \mathrm{mol} / \mathrm{l})$ & $80.70 \pm 23.46^{*}$ & $74.11 \pm 13.63$ & $44.2-106.1$ \\
\hline Glomerular filtration rate $(\mathrm{ml} / \mathrm{s})$ & $1.32 \pm 0.23$ & $1.41 \pm 0.17$ & $1.2-2.0$ \\
\hline
\end{tabular}

${ }^{*} p<0.05$ vs baseline (see Table 1 ). 


\subsection{Six Months}

Table 4 shows clinical and biochemical features at six months in $+D$ and $-D$ patients. As reported, no significant differences were found among the two groups. However, compared to baseline, we confirmed a significant reduction in serum glucose $(p<0.03), \mathrm{HbA1c}(p<0.002)$ and DBP levels $(p<0.009)$ in $+D$ patients. Moreover, in the same group, we registered an improvement in SBP values $(p<0.05)$, while no differences were found in parameters of lipid metabolism, creatinine levels and glomerular filtration rate, compared to baseline. Furthermore, as expected, $+D$ patients showed decreased serum PTH $(p<0.002)$ and increased 250HD ( $p<0.0001)$, phosphate $(p<0.002)$ and urinary calcium excretion $(p<0.004)$ compared to baseline. Weightadjusted urinary calcium excretion remained within reference range in all $+D$ patients. Once again, no significant variations were registered in $-D$ patientsvs baseline.

Table 4 BMI, blood pressure levels, parameters of glucose and lipid metabolism, renal function and parameters of calcium mineral metabolism in type 2 diabetic patients with $(+D)$ and without $(-D)$ vitamin $D$ supplementation at six months.

\begin{tabular}{|c|c|c|c|}
\hline & $\begin{array}{l}\text { +D patients } \\
(\mathrm{n}=16)\end{array}$ & $\begin{array}{l}\text {-D patients } \\
(n=14)\end{array}$ & $\begin{array}{l}\text { Reference } \\
\text { range }\end{array}$ \\
\hline $\mathrm{BMI}\left(\mathrm{kg} / \mathrm{m}^{2}\right)$ & $29.6 \pm 3.9$ & $30.5 \pm 3.3^{\dagger}$ & $18.5-24.9$ \\
\hline Systolic blood pressure (mmHg) & $149.7 \pm 16.2^{*}$ & $152.1 \pm 22.3$ & $90-140$ \\
\hline Diastolic blood pressure (mmHg) & $79.1 \pm 6.4^{*}$ & $82.9 \pm 6.7$ & $60-90$ \\
\hline Glucose (mmol/l) & $8.15 \pm 1.57^{*}$ & $8.23 \pm 1.78$ & $4.0-5.9$ \\
\hline Glycosylated Hemoglobin $(\mathrm{mmol} / \mathrm{mol})$ & $63.4 \pm 11.6^{*}$ & $62.6 \pm 12.0$ & $20-38$ \\
\hline Total cholesterol (mmol/l) & $4.59 \pm 1.22$ & $4.31 \pm 1.39$ & $<5.2$ \\
\hline HDL cholesterol (mmol/l) & $1.23 \pm 0.33$ & $1.35 \pm 0.32$ & $\begin{array}{l}\geq 1.0(\mathrm{M}) \\
\geq 1.3(\mathrm{~F})\end{array}$ \\
\hline Triglycerides (mmol/l) & $1.53 \pm 0.8$ & $1.38 \pm 1.1$ & $<1.7$ \\
\hline LDL cholesterol ( $\mathrm{mmol} / \mathrm{l})$ & $2.44 \pm 0.79$ & $2.16 \pm 0.99$ & $<4.1$ \\
\hline Non-HDL cholesterol (mmol/l) & $3.36 \pm 1.28$ & $2.97 \pm 1.45$ & $<3.4$ \\
\hline Creatinine $(\mu \mathrm{mol} / \mathrm{l})$ & $76.54 \pm 24.68$ & $74.84 \pm 15.50$ & $44.2-106.1$ \\
\hline Glomerular filtration rate $(\mathrm{ml} / \mathrm{s})$ & $1.45 \pm 0.32$ & $1.51 \pm 0.40$ & $1.2-2.0$ \\
\hline Serum total calcium (mmol/l) & $2.4 \pm 0.1$ & - & $2.2-2.6$ \\
\hline Serum phosphate (mmol/l) & $1.1 \pm 0.1^{*}$ & - & $0.8-1.5$ \\
\hline Urinary calcium (mmol/die) & $5.0 \pm 2.7^{*}$ & - & $2.5-7.5$ \\
\hline Urinary phosphate (mmol/die) & $18.8 \pm 4.6$ & - & $12.9-32.3$ \\
\hline PTH (pmol/l) & $4.88 \pm 3.68^{*}$ & - & $1.6-6.9$ \\
\hline 25OHvitamin D (nmol/l) & $96.27 \pm 30.53^{*}$ & - & $75-125$ \\
\hline Total alkaline phosphatase (UI/I) & $68.6 \pm 27.5$ & - & $\begin{array}{l}53-128(\mathrm{M}) \\
42-141(\mathrm{~F})\end{array}$ \\
\hline
\end{tabular}

${ }^{*} \mathrm{p}<0.05$ vs baseline (see Table 1$){ }^{\dagger} \mathrm{p}<0.05$ vs three months (see Table 3 ).

No significant changes were found in $+D$ and $-D$ patients at six vs three months, except for a slight increase in BMI in the latter group $(p<0.04)$. 
Furthermore, as shown in Figure 1 and 2, we found a significant difference in glucose levels ($11.0 \% \pm 19.1$ vs $+7.4 \% \pm 19.7$, respectively; absolute values $-1.21 \pm 1.87 \mathrm{vs}+0.51 \pm 1.52 \mathrm{mmol} / \mathrm{l}$, respectively; $p<0.04)$ and SBP values $(-4.7 \% \pm 9.0$ vs $+2.9 \% \pm 12.1$, respectively; absolute values $7.8 \pm 14.9$ vs $+3.6 \pm 18.9 \mathrm{mmHg}$, respectively; $p<0.05$ ) when comparing the mean percentage changes (from baseline to six months) of glucose and blood pressure levels between $+D$ and $-D$ patients. The same analysis did not reveal significant differences in mean percentage changes of $\mathrm{HbA} 1 \mathrm{c}(-14.2 \% \pm 12.6$ vs $-5.4 \% \pm 16.3$, respectively; absolute values $-11.3 \pm 11.1$ vs $-3.6 \pm 11.5$ $\mathrm{mmol} / \mathrm{mol}$, respectively; $\mathrm{p}=\mathrm{NS}), \mathrm{DBP}(-5.2 \% \pm 7.0$ vs $-0.3 \% \pm 11.7$, respectively; absolute values $4.4 \pm 5.7$ vs $-1.1 \pm 9.8 \mathrm{mmHg}$, respectively; $p=\mathrm{NS}$ ) and parameters of lipid metabolism (data not shown) between $+\mathrm{D}$ and $-\mathrm{D}$.

Moreover, the changes in anti-diabetic medications throughout the study did not differ among the two groups. Further, we did not register any variation in anti-hypertensive therapy.

Univariate analysis between calcium metabolism parameters and those of either glucose and lipid metabolism or hypertension at six months showed an inverse correlation between $250 \mathrm{HD}$ and $\mathrm{HbA} 1 \mathrm{c}$ levels ( $\mathrm{R}-0.66, \mathrm{p}<0.01$, Figure 3 ).

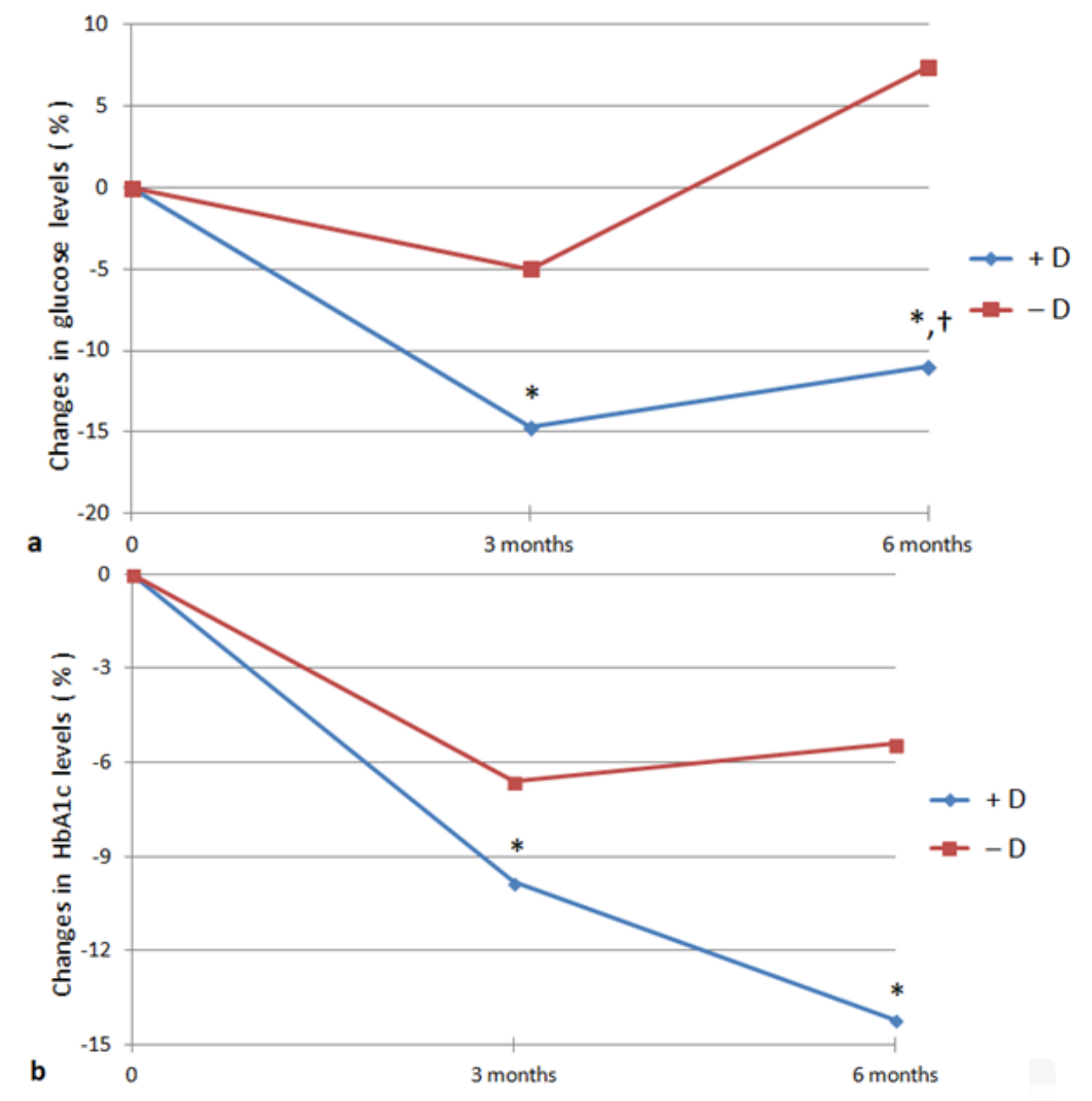

Figure 1 Mean percentage change in glucose (a) and glycosylated hemoglobin. (HbA1c, b) levels in type 2 diabetic patients with $(+D)$ and without (-D) vitamin $D$ supplementation after three and six months. ${ }^{*} p<0.05$ vs baseline; ${ }^{\dagger} p<0.04$ vs $-D$. 

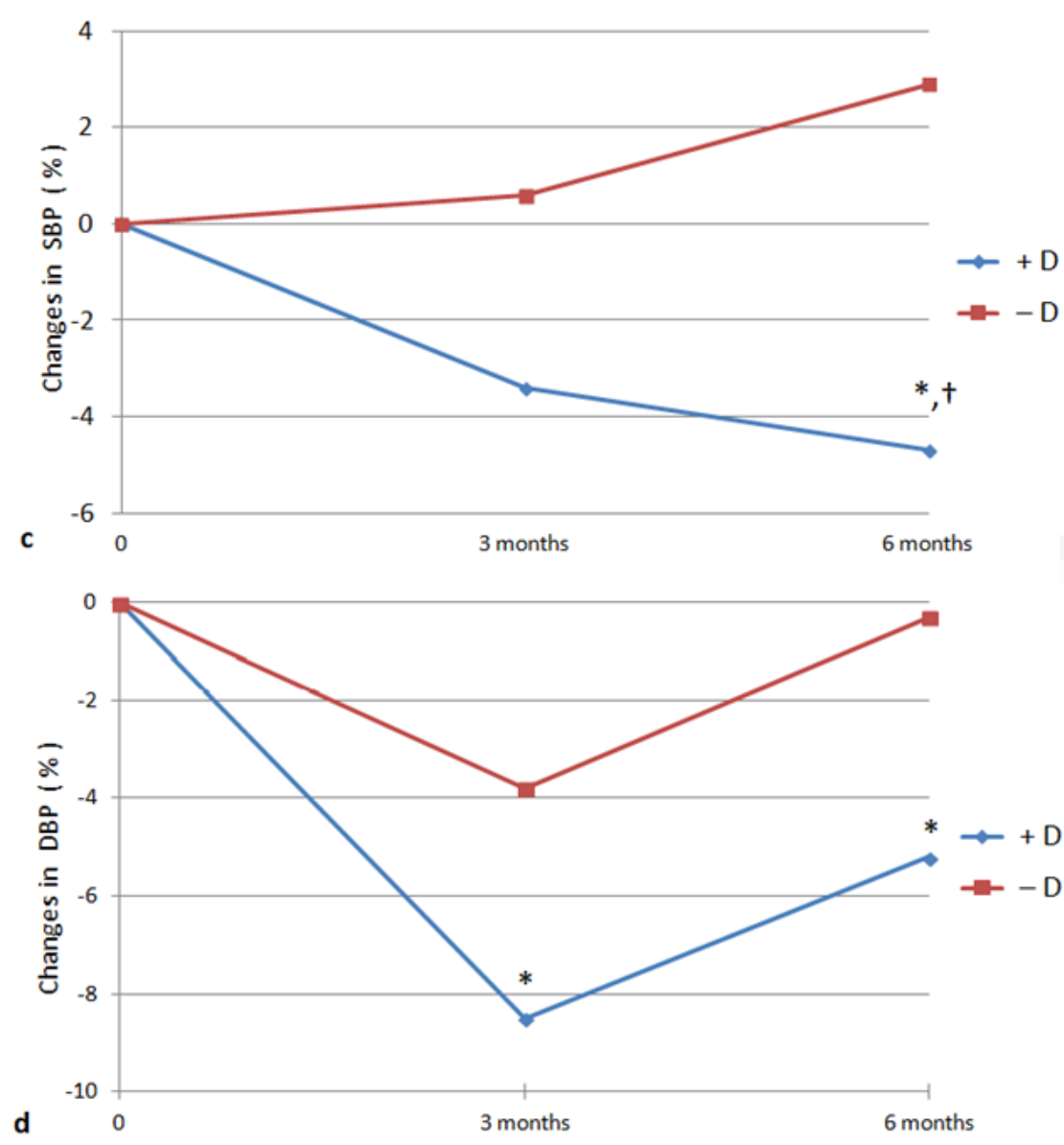

Figure 2 Mean percentage change in systolic (SBP, c) and diastolic blood pressure (DBP, d) levels in type 2 diabetic patients with $(+D)$ and without (-D) vitamin $D$ supplementation after three and six months. ${ }^{*} p<0.05$ vs baseline; ${ }^{+} p<0.05$ vs $-D$.

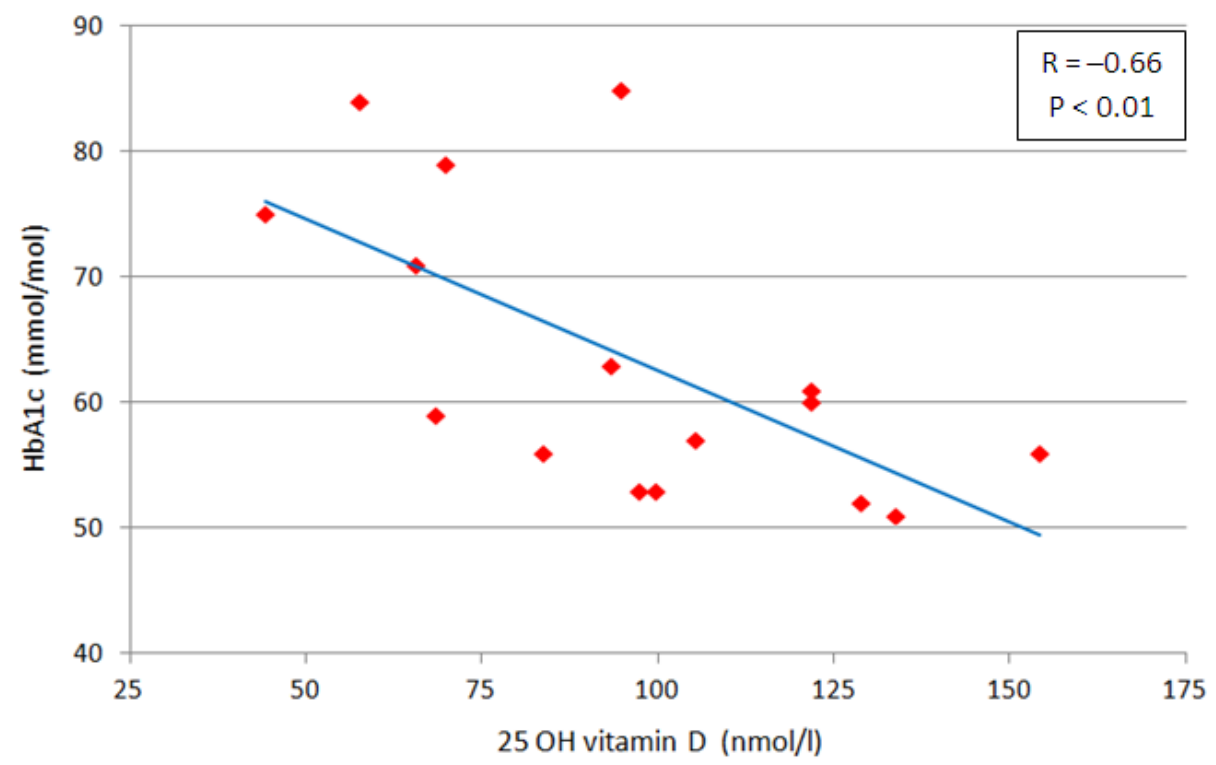

Figure 3 Univariateanalysisbetween $25 \mathrm{OH}$ vitamin D and glycosylated hemoglobin (HbA1c) levels in type 2 diabetic patients on vitamin $D$ supplementation at six months. 


\section{Discussion}

The results of our open-label randomized controlled pilot study in T2DM and severe vitamin D deficiency show that six-months vitamin $D$ supplementation has a beneficial effect on fasting glucose and SBP control, and lead to favorable but not significant changes in serum HbA1C and DBP levels. Moreover, patients supplemented with vitamin $D$ show a significant reduction of fasting glucose, HBA1c, SBP and DBP levels at six months in comparison to baseline. In addition, after sixmonths supplementation, we noticed an inverse relationship of serum 25OHD levels with HbA1c.

Hypovitaminosis D and cardio-metabolic risk factors such as T2DM, hypertension and dyslipidemia are highly prevalent in aging population. Despite the advances in the management of these chronic disorders, it is still hard to achieve an optimal disease control for many reasons including incorrect lifestyle and low treatment adherence. In this scenario, the opportunity to explore the role of a potential modifiable risk factor for cardio-metabolic alterations such as hypovitaminosis $D$ has attracted the interest of many researchers $[1,6]$. However, the effect of vitamin D supplementation on cardio-metabolic outcomes still remains a matter of debate. Two main mutually exclusive points of view are now competing in the field to explain this relationship. On one side, some Authors deem that vitamin $D$ is a marker of good health and nutrition so that hypovitaminosis $\mathrm{D}$ in patients with cardio-metabolic alterations could be a consequence rather than the cause of these disorders [17]. On the other side, many Authors support a causative role of vitamin $D$ deficiency in the development of hypertension and abnormalities of glucose and lipid metabolism [1, 2]. This view bases on plausible biological premise such as the presence of vitamin $D$ receptor and specific enzymes able to locally synthesize active vitamin D in many tissues involved in the regulation of glucose and lipid metabolism as well as in blood pressure control [18]. Accordingly, many epidemiological studies associate low vitamin $D$ levels with a wide range of cardio-metabolic disorders [2, 19]. However, specific randomized controlled trials designed to study the effect of vitamin $D$ supplementation on these outcomes have reported inconsistent results $[6,7,9,20,21,22]$. Nevertheless, it has to be highlighted that many flaws may be identified in these studies, such as a wide heterogeneity of studied patients' characteristics (i.e. age, body weight, metabolic control, vitamin D status) and vitamin D supplementation (form, route, dose, frequency and duration of administration). In light of these considerations, we decided to perform a well-designed randomized controlled pilot study, specifically focused on type 2 diabetic patients with comorbidity conditions such as hypertension and dyslipidemia, with poor glycemic control and severe vitamin $D$ deficiency, using a dosage of vitamin $D$ carefully weighed to achieve normal 250 HD levels. In doing so, we were able to demonstrate a slight favorable effect of vitamin D supplementation on fasting glucose and SBP levels. Although vitamin D supplementation produces only a slight effect on individual glycemic and blood pressure control in type 2 diabetic patients with hypovitaminosis $D$, we hypothesize that, from a population perspective, this approach could lead to reduction of cardiovascular morbidity and mortality [23]. Noteworthy, a wider and stronger positive effect of vitamin D supplementation, including also $\mathrm{HbA1C}$ and DBP, was noted in $+D$ patients comparing data at six months vs baseline. Though serum $25(\mathrm{OH}) \mathrm{D}$ was not re-measured in -D group, it is conceivable to assume that a negligible change could occur without vitamin $D$ supplementation in these elderly patients. However, we cannot exclude a slight improvement of vitamin $D$ status in this group, possibly due to better compliance with lifestyle as a consequence of participation in the study; if this was the case, we speculate that it could have prevented the 
attainment of a significant difference in changes of $\mathrm{HbA} 1 \mathrm{c}$ and diastolic blood pressure levels between $+D$ and $-D$ groups.

In line with our results, a meta-analysis that included longitudinal studies and randomized controlled trials (RCTs) reported a small improvement of fasting glucose and insulin resistance but no beneficial effect of vitamin D supplementation on $\mathrm{HbA1c}$ in patients with abnormal glucose tolerance [7]. It has to be noted that these results were obtained despite the studies reported in this meta-analysis were heterogeneous in terms of study subjects, including either those with impaired fasting glucose or impaired glucose tolerance or T2DM, and dose of supplemental vitamin $D$, duration of follow-up, generally of short-time period, and baseline 250HD levels, within reference range in half of the studies. Moreover, the number of eligible trials was also small and data on HbA1C, a better marker of glycemic status, were available only from few studies. In addition, a more recent systematic review [8] including prospective studies and randomized controlled trials of short-term duration ( $<3$ months) showed an improvement of glucometabolic parameters such as HbA1c, HOMA-B (an index of insulin secretion), HOMA-IR and QUICKI (indices of insulin resistance) after vitamin D supplementation in T2DM. Also, in long-term studies (> 3 months) a positive effect of vitamin $D$ supplementation on some glucometabolic outcomes was observed in type 2 diabetic vitamin D deficient patients [8].

On the contrary, the meta-analysis by Seida et al. [9], including studies of vitamin D supplementation in established T2DM, found non-significant improvement of HOMA-IR and HBA1c. Despite exclusion of non-RCT longitudinal studies and studies in which synthetic vitamin D preparations or vitamin D2 were used, it has to be noted that only few studies included in this meta-analysis administered large and prolonged dosages of vitamin $D$ and investigated severe vitamin D deficient patients. In addition, some trials included in this meta-analysis had not studied vitamin D related glycemic outcomes as their primary analysis.

Overall, these systematic reviews and meta-analysis highlight the slight beneficial effect of vitamin $D$ on glycemic outcomes in patients with abnormal glucose tolerance or uncontrolled T2DM with vitamin D deficiency and documented increase in 25OHD levels after supplementation.

Accordingly, vitamin $D$ is able to regulate expression of insulin and insulin receptor genes, as suggested by animal studies showing that calcitriol increases growth and differentiation of pancreatic beta cells and insulin secretion [24]. Moreover, vitamin D deficiency is related to insulin resistance and glucose intolerance [25] through both direct and indirect mechanisms including inflammation, down-regulation of peroxisome proliferator activated receptor-delta, increased activity of renin-angiotensin-aldosterone system (RAAS), due to enhanced expression of renin gene [26].

In addition, our findings show a slight favorable effect of cholecalciferol supplementation on SBP levels, whereas the beneficial effect on DBP does not reach statistical significance. Accordingly, a very recent meta-analysis [6] showed that vitamin $D$ supplementation is able to improve cardiovascular risk factors. Specifically, vitamin D supplementation, with doses above 4,000 IU/d and increased serum $250 \mathrm{OH}$ concentrations $\geq 86 \mathrm{nmol} / \mathrm{L}$ decreased SBP and DBP, serum PTH and serum hs-CRP. In particular, the only included study centered on hypertensive patients [27] found a significant reduction in blood pressure following five-months vitamin D supplementation (3,000 IU/day) with improved serum $250 \mathrm{HD}$ levels ( $50 \mathrm{nmol} / \mathrm{L}$ increase) compared to placebo. It has to be noted that the increase in $250 \mathrm{HD}$ levels reached by this study is lesser than ours, according to a lower administered dosage, but, including patients with higher 250HD baseline levels, lead to 
higher final values. In line with these results, Tabesh et al. [20], registered a statistically significant reduction of SBP values in vitamin $D$ insufficient type 2 diabetic patients randomized to weekly vitamin D (cholecalciferol $50.000 \mathrm{UI}$ ) and daily carbonate calcium (1000 mg) supplements, but also in those who took only vitamin D for eight weeks. Despite a better blood pressure profile (SBP 121$122 \mathrm{mmHg}$ ) in comparison with our patients $(157.5 \mathrm{mmHg})$, their higher dosage of cholecalciferol led to a similar absolute decrease in SBP values (approximately $7-8 \mathrm{mmHg}$ ). Moreover a similar but not significant effect was found also in terms of reduction of DBP values $(4-5 \mathrm{mmHg}$ in both studies). On the contrary, Jorde et al [21] did not reveal a significant effect of high dosage of cholecalciferol (40.000 UI once a week for 6 months) on blood pressure in type 2 diabetic patients. However, it has to be noted that these Authors enrolled patients with vitamin $D$ mild insufficiency (mean $250 \mathrm{HD}$ values equal to $60 \mathrm{nmol} / \mathrm{l}$ ).

Taken together, the above-mentioned studies show a favorable effect of vitamin D supplementation on blood pressure levels in severe vitamin D deficiency. The biological plausibility of vitamin D involvement in blood pressure control, is supported by the well known effects of vitamin D on endothelial cells and on RAAS activity $[26,28]$.

Further, we do not notice any effect of normalization of vitamin D status on serum lipid levels. In line with a meta-analysis by Wang et al [22], vitamin D seems to exert subtle effects on lipid serum profile. Probably our small sample size did not allow us to reach any statistical significance. However, the above-mentioned meta-analysis by Mirhosseini et al [6] found improved lipid profile (total cholesterol, triglyceride, HDL and LDL) after vitamin D supplementation. It has to be noted that many trials reported in this study included subjects with poor lipid control. On the contrary, our patients showed a good lipid profile at baseline, partly due to a wide use of anti-dyslipidemic drugs, so this could have hampered the effect of vitamin D supplementation on parameters of lipid metabolism. Overall, the few available studies to date do not allow to draw any definite conclusion about the effect of vitamin $D$ supplementation on lipid profile. The potential beneficial effects of vitamin D on lipid metabolism could be due to improvement of insulin secretion and sensitivity with reduced hepatic triglycerides synthesis and high serum HDL-cholesterol levels.

Furthermore, we hypothesize that favorable effect of vitamin D supplementation on cardiometabolic parameters might be, at least in part, due to PTH levels normalization. In fact, in our sample we register a higher prevalence of secondary hyperparathyroidism corrected by vitamin D supplementation. Many studies suggest that PTH is able to modulate glucose and lipid metabolism as well as blood pressure homeostasis $[29,30,31]$ and this hypothesis has been confirmed in models of chronic hypersecretion of PTH in which an increased prevalence of T2DM and metabolic syndrome have been reported [32].

Strengths of our study are the selection of an homogeneous population of uncontrolled noninsulin treated T2DM patients with comorbidities, such as hypertension and dyslipidemia, and with severe deficient vitamin D status; the randomized controlled study design; the administration of doses of cholecalciferol such as to reach levels of vitamin D sufficiency; control of dietary calcium intake with calcium salts administration whenever indicated; measurement of calcium mineral metabolism parameters including PTH, known to influence glucose and lipid metabolism and blood pressure control.

Our study has several limitations. Firstly the small sample size, consistently with the pilot nature of the study; secondly, the lack of a placebo formulation allowing an adequate concealment to perform a double blind randomized controlled trial; in addition, the short-term period of follow up; 
finally, the lack of control of the dietary intake of vitamin D and the lack of calcium mineral metabolism parameters in $-D$ patients at six months.

\section{Conclusions}

Our data in patients with poor controlled T2DM and severe vitamin D deficiency, indicate that six-months supplementation of cholecalciferol, able to restore normal 250HD levels, improves fasting glucose and SBP values and leads to favorable changes in $\mathrm{HbA1C}$ and DBP values. This slight improvement in glycemic and blood pressure control in type 2 diabetic patients could potentially reduce cardiovascular morbidity and mortality. Some long-term RCTs are still in progress in order to clarify the effect of vitamin D supplementation on cardio-metabolic disorder and micro- and macrovascular outcomes with results expected in the next years.

\section{Author Contributions}

All the Authors participated in the concept, design, analysis, writing and revision of the manuscript. This material or similar material has not been and will not be submitted to or published in any other publication.

\section{Funding}

None

\section{Competing Interests}

The authors have declared that no competing interests exist.

\section{References}

1. Awad $A B$, Alappat $L$, Valerio $M$. Vitamin $D$ and metabolic syndrome risk factors: Evidence and mechanisms. Crit Rev Food Sci Nutr. 2012; 52: 103-112.

2. Parker J, Hashmi O, Dutton D, Mavrodaris A, Stranges S, Kandala NB, et al. Levels of vitamin D and cardiometabolic disorders: Systematic review and meta-analysis. Maturitas. 2010; 65: 225-236.

3. Isaia G, Giorgino R, Adami S. High prevalence of hypovitaminosis D in female type 2 diabetic population. Diabetes Care. 2001; 24: 1496.

4. Cigolini M, lagulli MP, Miconi V, Galiotto M, Lombardi S, Targher G. Serum 25-hydroxyvitamin D3 concentrations and prevalence of cardiovascular disease among type 2 diabetic patients. Diabetes Care. 2006; 29: 722-724.

5. Joergensen C, Gall MA, Schmedes A, Tarnow L, Parving HH, Rossing P. Vitamin D levels and mortality in type 2 diabetes. Diabetes Care. 2010; 33: 2238-2243.

6. Mirhosseini N, Rainsbury J, Kimball SM. Vitamin D Supplementation, Serum 25(OH)D concentrations and cardiovascular disease risk factors: A systematic review and meta-analysis. Front Cardiovasc Med. 2018; 5: 87. 
7. George PS, Pearson ER, Witham MD. Effect of vitamin D supplementation on glycaemic control and insulin resistance: A systematic review and meta-analysis. Diabet Med. 2012; 29: e142-150.

8. NigilHaroon N, Anton A, John J, Mittal M. Effect of vitamin D supplementation on glycemic control in patients with type 2 diabetes: a systematic review of interventional studies. J Diabetes Metab Disord. 2015; 14: 3.

9. Seida JC, Mitri J, Colmers IN, Majumdar SR, Davidson MB, Edwards AL, et al. Clinical review: Effect of vitamin D3 supplementation on improving glucose homeostasis and preventing diabetes: A systematic review and meta-analysis. J Clin Endocrinol Metab. 2014; 99: 35513560.

10. Jorde R, Grimnes G. Vitamin D and health: the need for more randomized controlled trials. J Steroid Biochem Mol Biol. 2015; 148: 269-274.

11. Leu M, Giovannucci E. Vitamin D: epidemiology of cardiovascular risks and events. Best Pract Res Clin Endocrinol Metab. 2011; 25: 633-646.

12. Heaney RP, Davies KM, Chen TC, Holick MF, Barger-Lux MJ.Human serum 25hydroxycholecalciferol response to extended oral dosing with cholecalciferol. Am J ClinNutr. 2003; 77: 204-210.

13. Ross AC, Manson JE, Abrams SA, Aloia JF, Brannon PM, Clinton SK, et al. The 2011 report on dietary reference intakes for calcium and vitamin $D$ from the Institute of Medicine: What clinicians need to know. J Clin Endocrinol Metab. 2011; 96: 53-58.

14. National Cholesterol Education Program (NCEP) Expert Panel on Detection, Evaluation, and Treatment of High Blood Cholesterol in Adults (Adult Treatment Panel III). Third report of the National Cholesterol Education Program (NCEP) expert panel on detection, evaluation, and treatment of high blood cholesterol in adults (Adult Treatment Panel III) final report. Circulation. 2002; 106: 3143-3421.

15. ESH/ESC Task Force for the Management of Arterial Hypertension. Practice guidelines for the management of arterial hypertension of the European Society of Hypertension (ESH) and the European Society of Cardiology (ESC). J Hypertens. 2013; 31: 1925-1938.

16. Holick MF. Vitamin D deficiency. N Engl J Med. 2007; 357: 266-281.

17. Autier P, Boniol M, Pizot C, Mullie P. Vitamin D status and ill health: A systematic review. Lancet Diabetes Endocrinol. 2014; 2: 76-89.

18. Christakos S, Hewison M, Gardner DG, Wagner CL, Sergeev IN, Rutten E, et al. Vitamin D: Beyond bone. Ann N Y Acad Sci. 2013; 1287: 45-58.

19. Wang TJ. Vitamin D and Cardiovascular Disease. Annu Rev Med. 2016; 67: 261-272.

20. Tabesh M, Azadbakht L, Faghihimani E, Tabesh M, Esmaillzadeh A. Effects of calcium-vitamin D co-supplementation on metabolic profiles in vitamin $D$ insufficient people with type 2 diabetes: a randomised controlled clinical trial. Diabetologia. 2014; 57: 2038-2047.

21. Jorde R, Figenschau Y. Supplementation with cholecalciferol does not improve glycaemic control in diabetic subjects with normal serum 25-hydroxyvitamin D levels. Eur J Nutr. 2009; 48: 349-354.

22. Wang $H$, Xia N, Yang Y, Peng DQ. Influence of vitamin D supplementation on plasma lipid profiles: a meta-analysis of randomized controlled trials. Lipids Health Dis. 2012; 11: 42. 
23. Bjelakovic G, Gluud LL, Nikolova D, Whitfield K, Wetterslev J, Simonetti RG, et al. Vitamin D supplementation for prevention of mortality in adults. Cochrane Database Syst Rev. 2014; 1: CD007470.

24. Mitri J, Pittas AG. Vitamin D and diabetes.EndocrinolMetabClin North Am. 2014; 43:205-232.

25. Chiu KC, Chu A, Go VL, Saad MF. Hypovitaminosis D is associated with insulin resistance and beta cell dysfunction. Am J Clin Nutr. 2004; 79: 820-825.

26. Harinarayan CV. Vitamin D and diabetes mellitus. Hormones (Athens). 2014; 13: 163-181.

27. Larsen T, Mose FH, Jesper N. Bech JN, Hansen AB, Pedersen EB. Effect of cholecalciferol supplementation during winter months in patients with hypertension: a randomized, placebocontrolled trial. Am J Hypertens. 2012; 25: 1215-1222.

28. Kassi E, Adamopoulos C, Basdra EK, Papavassiliou AG. Role of vitamin D in atherosclerosis. Circulation. 2013; 128: 2517-2531.

29. Fadda GZ, Akmal M, Lipson LG, Massry SG. Direct effect of parathyroid hormone on insulin secretion from pancreatic islets. Am J Physiol. 1990; 258: E975-984.

30. Chiu KC, Chuang LM, Lee NP, Ryu JM, McGullam JL, Tsai GP, et al. Insulin sensitivity is inversely correlated with plasma intact parathyroid hormone level. Metabolism. 2000; 49: 1501-1505.

31. Zhang Y, Zhang DZ. Circulating parathyroid hormone and risk of hypertension: A meta-analysis. Clin Chim Acta. 2018; 482: 40-45

32. Procopio $M$, Barale $M$, Bertaina S, Sigrist S, Mazzetti R, Loiacono $M$, et al. Cardiovascular risk and metabolic syndrome in primary hyperparathyroidism and their correlation to different clinical forms. Endocrine. 2014; 47: 581-589.

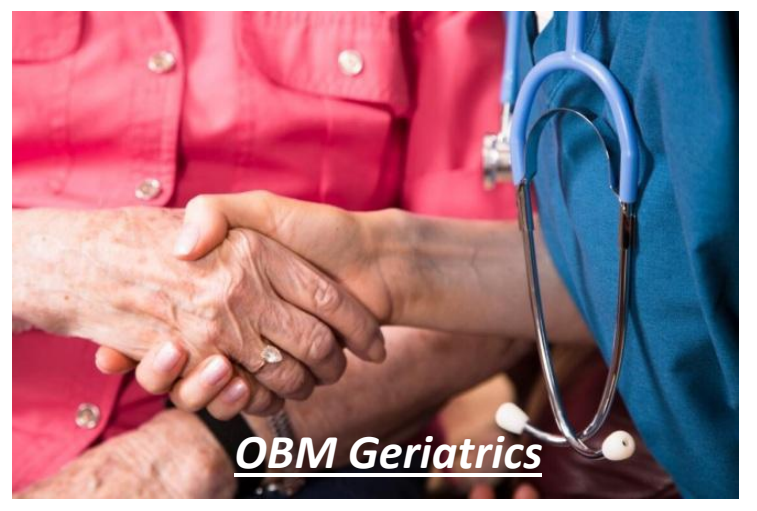

Enjoy $O B M$ Geriatrics by:

1. Submitting a manuscript

2. Joining in volunteer reviewer bank

3. Joining Editorial Board

4. Guest editing a special issue

For more details, please visit: http://www.lidsen.com/journals/geriatrics 
Research Article

\title{
Key Dimensions of Therapeutic Lies in Dementia Care: A New Taxonomy
}

Rachel Mills ${ }^{1,+}$, Louisa Jackman ${ }^{2,+}$, Mithila Mahesh ${ }^{1}$, lan James ${ }^{3,+, *}$

1. Doctorate in Clinical Psychology, University of Newcastle, United Kingdom; E-Mails: Rachel.mills@ntw.nhs.uk; m.v.mahesh2@newcastle.ac.uk

2. Leeds Teaching Hospitals Trust, United Kingdom; E-Mail: louisa.jackman@nhs.net

3. Campus of Ageing and Vitality, Northumberland Tyne and Wear (NTW) NHS, Westgate Road, Newcastle upon Tyne, United Kingdom; E-Mail: lanAndrew.James@ntw.nhs.uk

$\dagger$ These authors contributed equally to this work.

* Correspondence: Ian James; E-Mail: lanAndrew.James@ntw.nhs.uk

Academic Editor: Miyako Tazaki

Special Issue: Treatment of Dementia

OBM Geriatrics

2019, volume 3, issue 1

doi:10.21926/obm.geriatr.1901032
Received: December 12, 2018

Accepted: January 30, 2019

Published: February 12, 2019

\begin{abstract}
Background: Research suggests that the use of lies and deception is prevalent within dementia care settings, despite ongoing debates raised about the ethics of this approach. There has been increasing exploration of when and why deceptive practices should be used, but the lack of clarity as to what constitutes a lie has caused difficulty in ensuring that lies are used ethically. The aim of this study was to widen our understanding of the key dimensions that underpin the use of lies, and further to use this information to develop a taxonomy of lies within dementia care settings.

Methods: A mixed methods approach was used for the study, which consisted of three phases: (1) obtaining examples of lies from experienced clinicians, (2) using expert advisors to standardise the examples, (3) asking independent participants to sort the examples into similarly themed groups. Hierarchical cluster analysis was used to produce clusters which led to the development of the taxonomy.
\end{abstract}

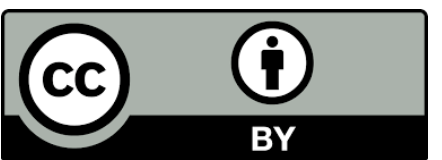

(C) 2019 by the author. This is an open access article distributed under the conditions of the Creative Commons by Attribution License, which permits unrestricted use, distribution, and reproduction in any medium or format, provided the original work is correctly cited. 
Results: The results indicated that lies are mainly used in the best interests of people with dementia, often to reduce distress or manage difficult behaviour. From the developed taxonomy, there were two overarching clusters: the larger cluster was concerned with the welfare or activity of a family member/friend, and the second cluster was concerned with the welfare or activity of the person themselves.

Conclusions: These results highlighted that in order to use lies appropriately it was important for caregivers to have a good understanding of the person with dementia and their life experiences. An awareness of the potential problems in using deception is key, and it is recommended that communication training should be employed to support carers in the use of therapeutic lying as a person-centred and beneficial approach. The study also shows that lies are often used to deal with the needs of PWD who are expressing concerns about the wellbeing of others. This notion of the importance of 'other-directed' needs in PWD is a new and relevant finding.

\section{Keywords}

Dementia; therapeutic lies; communication; taxonomy

\section{Introduction}

Recent literature suggests that lying and deception are prevalent within dementia care settings, with $96 \%$ of care staff admitting to using lies [1]. In line with the Mental Capacity Act 2005 [2], James et al. (2006) developed guidelines for staff when using lies with people with dementia (PWD) $[1,2]$. Literature suggests that when lies are used in care settings, it is generally judged as beneficial for the wellbeing of the PWD, and therefore in their best interests $[1,3,4]$. Lies are employed by carers in an attempt to communicate sensitively with PWD who have become timeshifted (i.e. viewing the past as the current reality). During such times, the person with dementia may believe certain altered realities, i.e. a deceased spouse is still alive, he is still working at a job, or she is living independently at home [5]. In such situations, lies may be deemed acceptable in order to prevent unnecessary distress [6].

Blum (1994) researched the types of lies used within family homes, and developed the only taxonomy in the area [7]. Her taxonomy consisted of four main classes of deception: "going along with an incorrect assumption", "not telling," "little white lies," and "tricks" [7].

Blum's work was helpful, but limited to the views of family carers [7]. Recent studies suggested that lies used in care settings may be different [8]. Indeed, the developing literature highlighted a lack of clarity and consistency regarding the nature of therapeutic lies employed in these settings [9]; hence, the need for the current study.

Recent work suggests that therapeutic lies are consistent with Algase's (1996) notion of a 'needs-led' intervention [10]; that is a person centred intervention that attempts to meet the unmet needs of clients [11]. Therefore therapeutic lies can be regarded as person centred interventions to be used and documented in people's care plans [11]. Further, as person-centred care has become the ethos of the approaches delivered in $24 \mathrm{hr}$ care settings [12], it is important to further understand the types of lies being used and their roles in communication strategies. A 
major review of lies as communication techniques has recently been undertaken by the Mental Health Foundation [6]. It concluded that there were five typical methods of responding to difficult questions posed by PWD: (1) telling the whole truth, (2) looking for an alternative meaning to the question and responding accordingly, (3) distracting the person from the question, (4) going along with PWD's perspectives, and (5) lying [6]. MHF recommended starting from position (1) whenever possible, and using lies as the last resort in order to prevent unnecessary distress [6].

The present study aimed to develop an empirically derived taxonomy of lies used within residential dementia care settings and to understand the key dimensions that underpin this. It is envisaged that the resulting taxonomy will be a useful tool to support the understanding of lies and could be used within care settings to develop effective communication with PWD.

\section{Methods}

\subsection{Design}

A mixed methodology was selected, enabling the researchers to obtain detailed information of participants' experiences of lies [13]. The study contained three data collection phases: (1) gathering examples of lies from experienced clinicians, (2) standardising 'examples' with support from experienced clinicians, (3) employing independent participants for a sorting task to place the 'examples' into piles of similarity. Analysis was then conducted using cluster analysis.

Phase One - The first phase consisted of a survey of lies witnessed in care home settings. The data was gathered from expert clinicians in dementia care with experience of observing lies in residential settings. They were recruited from Older Adult Challenging Behaviour Teams in Northumberland Tyne and Wear (NTW) NHS Foundation Trust. Through NTW, team managers approached clinicians to inform them of the study, and 17 participants consented to participate in this initial phase (15 female, $88 \%$ ). All of these clinicians regularly worked into care homes with PWD, with a range of experience of 37-151 months working into such settings. Participants were asked to provide examples of lies that they had witnessed being told to PWD by care staff in residential homes. They were asked to record the lies using a specific format, to ensure that the required data was gathered from each lie. Participants were asked to describe: the situation requiring a lie, what was said, and the perceived intention of the liar. Each participant provided two to three examples, generating a total of 45 examples of lies.

Phase Two - The second phase consisted of content revision of the 45 examples by expert advisors, to standardise material in preparation for the sorting task in Phase Three. Two local female clinicians, different to those recruited in Phase One, were recruited as expert advisors from Older Adult services in the North East of England. Inclusion criteria was that participants had experience in the subject matter, but were not working within NTW Challenging Behaviour services. The expert advisors discussed standardising the examples with consideration toward relevant information required for unbiased sorting. The expert advisors also provided advice on whether data saturation had been achieved from the 45 examples.

Phase Three - The third phase included a sorting task in which participants were asked to read through the 45 examples and sort them into piles of perceived similarity. Trainee clinical psychologists from the Newcastle University Clinical Psychology Doctorate course were contacted by course directors via email with information regarding the current study. Following this, 10 
trainees consented to participate ( 8 female). Inclusion criteria required trainees to have completed a placement within Older Adult services so they had experience in dementia care. Participants were asked to sort the lies into piles which were similar to each other in some way. Participants were asked to describe why lies had been placed in each pile, and to attach a concise label to each pile.

\subsection{Analysis}

A proximity matrix was developed from the piles provided by participants in Phase Three. This was done by calculating how often each lie was paired with each of the other 44 . This process produced a number between 0 to 10 ( 0 being never paired, 10 being paired by all participants) in each column, showing how many times each lie had been paired with another (i.e. lie 1 with lie 34).

Hierarchical cluster analysis was employed. Analysis was conducted using the SPSS software program (Statistical Package for the Social Sciences; IBM, 2015). The cluster method used was average/between groups' linkage along with an interval measure of Euclidean distance.

\subsection{Ethics}

The project was approved by Newcastle University Faculty of Medical Sciences Ethics Committee and was registered with Northumberland Tyne and Wear Mental Health Trust Research and Development Department on $25^{\text {th }}$ May 2016.

\section{Results}

Table 1 illustrates the variety of situations (and frequency) that lies occurred. From the 45 examples provided, $80 \%$ of situations were related to disorientation (to time and/or place) of the PWD. In addition, over half (64\%) lies were related to the PWD being concerned for/about another person (i.e. wanting to collect their 'grown-up' children from school, asking for deceased parents).

Table 2 demonstrates the perceived intention of the liar as judged by the clinicians providing the examples in Phase One. In some situations, there was more than one perceived intention (i.e. to reduce distress when meeting personal care needs). $96 \%$ of intentions were in the best interest of the PWD, $4 \%$ were perceived to be for the benefit of someone else (i.e. to reduce distress of family). There were no instances in which phase 1 participants judged carers to be employing lies for their own benefit.

Phase 1 participants revealed that 13 of the 'examples' of lies were included in a care plan for the PWD.

Table 3 shows the results of the cluster analysis. There were two primary clusters, with five sub-clusters within these. After examination of lies in these clusters, themes were extrapolated. Labels were assigned to each of the five sub-clusters to categorise the type of lies incorporated within each cluster. These labels were given by the first author, with guidance from the co-authors, and were not obtained directly from 'wording' from the data sets. 
Table 1 Overview of examples of situations where lies were used in care homes.

\author{
Situation that triggered lie \\ PWD wants to see/contact \\ deceased relative \\ PWD believes their children \\ are still school-age \\ PWD wants to see or \\ contact family \\ PWD not allowing staff to \\ provide personal care \\ PWD believes they are still \\ employed and wants to go \\ to work \\ Delusion relating to \\ perception of objects
}

PWD believes they still live at their previous home and wishes to return

PWD believed themselves to be a prisoner in the care home

Visiting family were leaving

\section{Examples of lies}

Resident pacing, dressed to go out and asking to go and see her mother (deceased)

Resident wanting to leave care home to pick up children from school

Resident repeatedly requesting to telephone wife (several times daily)

Resident requesting to telephone wife (several times daily)

Resident thinking she still had to go to work every day, trying to leave care home

Resident had a toy cat - he interacted with the cat as though it was a living pet; feeding it food from the dining table, naming it.

Resident wanting to go home (no longer lives at home, permanent resident in a care home)

Resident becoming

agitated/aggressive as asking to use phone to contact police as being kept as a prisoner

Family were leaving and the resident was becoming distressed

\section{Frequency of occurrence}

15

7

16

5

(\%)

33

4

9

9

4

9

3

7

2

4

1

2

45

Table 2 Overview of perceived intentions of lies from Phase one participants.

\section{Perceived intention}

Reduce agitation of the PWD

Provide reassurance to the PWD

Management of $\mathrm{BtC}$ of the PWD

To reduce anxiety/worry of the PWD

To ensure that personal care needs of the PWD were met

To reduce distress of a relative of the PWD

\section{Frequency}

27

15

14

10

4

3
Percentage (\%) 37

21

19

14

5

4 
Table 3 Categories of lies developed from analysis in Phase three.

\section{Primary \\ Cluster \\ Separation}

1

Non-client

related (lies

concerning

actions or

well-being of

another

person)

$$
\begin{aligned}
& \text { Sub-cluster - } \\
& \text { category label }
\end{aligned}
$$

1.1

Delayed

fulfilment of

request

\section{2}

Providing reassurance of

well-being

1.3

Explanation for absence

\section{Explanation}

Putting off requests by the

client to see a

person (often

deceased)

Assuring client

that a specific

person is safe,

when often

deceased (or

now grown

children)

False account of where an (often deceased)

relative is Examples of lies
included

"Your dad is

coming to get you

at 9.30am

tomorrow"

"The bairns are at school and will be

home soon, they're safe"

"Your mam's not at home at the moment, she's gone to the shops"

\section{Perceived Intention of type of lie}

Reducing immediate distress and managing subsequent BtC.

Reducing distress by providing reassurance to the PWD that the person in question is safe.

Reducing distress and managing of BtC (i.e. aggression) were intentions. This type of lie uses a more direct (and less reassuring) mechanism for care staff to diffuse a

\begin{tabular}{|c|c|c|}
\hline \multirow[t]{2}{*}{$\begin{array}{l}\text { Performing a } \\
\text { false role or } \\
\text { story to match } \\
\text { the client's } \\
\text { reality }\end{array}$} & $\begin{array}{l}\text { Staff engaged with } \\
\text { toy cat as though it } \\
\text { were real, e.g. } \\
\text { putting food out } \\
\text { for it at request of } \\
\text { resident }\end{array}$ & $\begin{array}{l}\text { Meeting PWDs' } \\
\text { personal care needs, } \\
\text { and reducing their } \\
\text { anxiety and } \\
\text { behaviours such as } \\
\text { wandering. }\end{array}$ \\
\hline & $\begin{array}{l}\text { "No need to go to } \\
\text { work this morning, } \\
\text { you're on holiday." }\end{array}$ & \\
\hline
\end{tabular}
difficult situation.

Used for the benefit of others, rather than for the PWD, employed to reduce distress for visiting relatives. 


\subsection{Non-Client Related (Lies Concerning Actions or Well-Being of Another Person)}

The key emerging theme was that all of the lies were used to assuage distress and anxiety resulting from disorientation of the PWD regarding the current status of someone important to them, i.e. whereabouts of a deceased family member. This was the larger of the two primary clusters.

\subsubsection{Delayed Fulfilment of Request.}

The five lies in this cluster concerned the PWD being told that a fictitious event would be occurring. These events were all linked to requested contact with a relative (often deceased), where the PWD was told that they would see that person at a specified time in the near future.

\subsubsection{Providing Reassurance of Well-Being}

The eight lies in this cluster related to reassuring the PWD of the safety and whereabouts of a relative they were concerned about (often already deceased or now grown children). In these cases, the PWD's distress were underpinned by a belief that they still held caring roles for the relative in question, and their absence meant they were not protecting and/or ensuring their safety.

\subsubsection{Explanation of Absence}

The nine lies in this category also linked to the PWD's anxiety and concerns related to the whereabouts of a relative, but the lies employed in these situations lacked the element of safety reassurance of the previous sub-cluster.

\subsection{Client Related (Lies Concerning Activity or Well-Being of the Client)}

Lies within this cluster were concerning issues regarding the PWD themselves rather than someone else, i.e. completion of personal care needs, or believing that they were still employed in their previous jobs. Situations requiring lies were related to disorientation of the PWD and consequent distress, but not in relation to a third person.

\subsubsection{Enactment}

The eight examples of lies here were the most varied regarding the approach used by care staff, but all encompassed a similar theme. Staff appeared to engage in the reality of the PWD to a greater degree in order to meet any of the specific needs of the PWD. For example, informing a PWD who wanted to go to work that he was on holiday.

\subsubsection{Fobbing Off}

The nine examples of lies in this cluster appeared to demonstrate less engagement with the subjective reality of the PWD, unlike the previous sub-clusters. These examples concerned the PWD wanting something (i.e. to use the telephone, looking for their car) and being given false information and/or promises to quickly 'shut down' the request. This is the only sub-cluster where 
the use of lies were for the benefit of others (e.g. for visiting relatives) rather than the PWD directly. The term 'fobbing off' was devised by the first author as she felt it best explained the theme; however, it was not a term that came up in vivo from the data.

\section{Discussion}

This study examined the key dimensions that underpin the use of lies in dementia care settings with the aim of developing a taxonomy of these lies. The majority of the situations in which care staff felt that deception was required was linked to the issue of disorientation of the PWD, and the disparity between their subjective reality and current reality. Within this disorientation, situations where deception was used were largely related to misplaced beliefs by the PWD, such as asking for deceased relatives. These findings support previous literature examining situations in which lies are often employed with PWD [14].

Perceived intentions of care staff when using lies with PWD were varied, but the reduction of agitation was judged to be the major theme, alongside providing reassurance, managing $\mathrm{BtC}$ and reducing anxiety of the PWD. These results suggest that for the most part, phase one participants perceive that care staff use lies to enhance well-being of the PWD with their best interests in mind; therefore, these types of lies could be viewed as therapeutic $[3,4,15]$.

For the small proportion of lies that were judged as less therapeutic or beneficial, perceived intentions were management of either BtC or the distress of relations. The only concerning category that emerged in the cluster analysis was 'fobbing off,' which suggested there were a category of lies used for the carers' benefits. Fobbing-off is likely to reflect the busy caregiver attempting to avoid complex interactions and explanations in order to allow themselves to get on with their own work. This notion would be consistent with anecdotal evidence of environments termed 'task focused cultures.'

It was argued in a previous study that those lies that can be regarded as 'therapeutic' should be termed 'person-centred' [16] because the lies are attempting to enhance the wellbeing of people with dementia. In the same article it was argued that staff should be trained to deliver therapeutic lies which meet the needs of PWD [16]. This needs-led approach to interventions is highlighted in many of the national dementia strategies [17]. However, when needs-led interventions are normally discussed, the foci of the need is always the person with dementia. Interestingly, the present study suggests that clinicians should throw their nets wider when using needs-led perspectives, taking account of the person's perceived needs of 'others' (ie. family and friends). An awareness of this feature is crucial because it is the PWD's concerns about the wellbeing of others that seem to be activating their queries and possible distress that ultimately lead to the use of lies.

Despite the use of lies in dementia care remaining controversial $[18,19]$, growing literature indicates that it is prevalent in these settings [1]. The findings of the current study suggest potential clinical implications around the use of lies. Firstly, in order to use lies in a person-centred way, care staff need to have a good understanding of the PWD and their unique experiences [20]. It may be beneficial for care home management to ensure that any use of lies are discussed and agreed upon within their clinical team as well as are fully documented and used consistently through use of care planning [6]. The current study found that a large proportion of lies relied on the PWD being unable to remember information at a later date; however, as awareness can fluctuate in PWD $[6,21]$ this should not be assumed. As communication is deemed one of the 
most important factors in provision of quality care [22], it may be useful for staff to receive specific training on the use of lies [23]. James and Caiazza have shown that clinical psychologists have particular skills in delivering such training programmes [16].

Though the developed sub-themes were in line with Blum's results [7], our taxonomy suggests two larger meta-themes: non-client related (lies concerning actions of well-being of another person i.e. family member); and client related (lies concerning actions of well-being of the PWD). This difference in findings may reflect the difference in settings in which the studies were conducted. Indeed, because the Blum study was conducted in family settings, there may have been less of a need for the PWD to enquire about the whereabouts of their family members [7]. Therefore, future directions may involve recruiting participants across a variety of health care settings and other environments, i.e family homes, to validate examples provided from the current study's taxonomy and understand if they are applicable and generalizable to other populations.

A major strength of the study was that the methodology used enabled inclusion of information regarding PWD in the later stages of the disease; a population who are often excluded from research [24]. Since, it is in the later stages of dementia that people are more likely to be lied to [25], it is important for this population to be represented within research on deception. The various limitations in the current study must also be noted. For example, the first phase of data collection was based on clinicians' responses, the accuracy of which obviously cannot be crosschecked. Additionally, though the current study maintained high standards of anonymity when collecting data, there could still have been a potential response bias in reporting because the clinicians may have been worried about being identified owing to the small number of participants. Therefore this may have led some clinicians to omit reporting of instances when lies were used when working with PWD. For instance, participants may have omitted examples of poor practice. In terms of analysis, the deterministic quality of hierarchical cluster analysis prevents reassessment after items are grouped together and clusters can be difficult to define if no clear separation exists [26], which was an issue within this study. As a result, identification of clusters relied on a subjective interpretation of the dendogram leaving results open to unconscious bias.

Owing to some of the concerns mentioned above about the biases in the use of 'reported' data, in the next phase of our work we are attempting to observe lies being used in-vivo. A Northumbria University PhD. student/qualified mental health nurse is currently collecting 'live' data as part of her postgraduate degree, and will be reporting her findings in the next two years.

\section{Conclusions}

This study explored the key dimensions underpinning the use of lies in dementia care settings to develop a taxonomy of lies. Findings indicated that lies are primarily used in the best interests of disoriented PWD, largely aiming to improve well-being by reducing distress and BtC. However, there were a group of lies which involved PWD being 'fobbed-off,' perhaps in an attempt to save carers' time and resources. The taxonomy identified two overarching clusters concerning the welfare of others and that of the PWD, containing five sub-clusters between them. The identified sub-clusters demonstrated the importance of understanding the unique perspective and experiences of each PWD, and how this can be used when employing lies. 


\section{Acknowledgments}

The authors wish to thank the participants of this study, and the staff of the Doctorate Course in Clinical Psychology, University of Newcastle who assisted with the development of the original proposal.

\section{Author Contributions}

Rachel Mills prepared the study for ethics, collected and analysed the data, and submitted a version of the study for her doctoral thesis.

Louisa Jackman contributed to the theoretical ideas and supervised the project.

lan James had the original idea for the study and supervised the project.

Mithila Mahesh assisted with revisions to the original thesis, and undertook the editing and preparation for submission.

\section{Funding}

This project was undertaken in part fulfilment of the Doctorate in Clinical Psychology, and no funding was sought.

\section{Competing Interests}

The authors have declared that no competing interests exist.

\section{References}

1. James IA, Wood-Mitchell AJ, Waterworth AM, Mackenzie LE, Cunningham J. Lying to people with dementia: Developing ethical guidelines for care settings. Int J Geriatr Psych. 2006; 21: 800-801.

2. The Stationery Office Limited. Mental Health Capacity. London: Queen's Printer of Acts of Parliament. 2005. https://www.legislation.gov.uk/ukpga/2005/9/contents

3. Tuckett AG. Stepping across the line: Information sharing, truth telling and the role of the personal carer in the Australian nursing home. Qual Health Res. 2007; 17: 489-500.

4. Tuckett AG. The experience of lying in dementia care: A qualitative study. Nurs Ethics. 2012; 19: 7-20.

5. Gibbons L, Keddie G, James IA. Investigating the phenomenon of time-shifting. Aust J Dement Care. 2018; 7: 32-34

6. Kirtley A, Williamson T. What is truth? An inquiry about truth and lying in dementia care. Mental Health Foundation. 2016. www. mentalhealth.org.uk.

7. Blum NS. Deceptive practices in managing a family member with Alzheimer's disease. Symbolic Interaction. 1994; 17: 21-36.

8. James I, Jackman L. Understanding Behaviour in Dementia that Challenges, Second Edition. London: Jessica Kingsley Publishers. 2017.

9. Caiazza R, James IA. Re-defining the Notion of the Therapeutic Lie: Person-centred Lying. FPOP Bulletin: 2015. 2015. 
10. Algase DL, Beck C, Kolanowski A, Whall A, Berent S, Richards K, et al. Need-driven dementia compromised behaviour: An alternative view of disruptive behaviour. Am J Alzheimers Dis. 1996; 11: 12-19.

11. O'Conner E, Caiazza R, James I. Use of the needs hierarchy framework to assist with the telling of person centred lies. J Dement Care. 2017; 25: 22-25.

12. Brooker D. What is person-centred care in dementia? Rev Clin Gerontol. 2003; 13: 215-222.

13. Michie S, Richardson M, Johnston M, Abraham C, Francis J, Hardeman W, et al. The behavior change technique taxonomy (v1) of 93 hierarchically clustered techniques: Building an international consensus for the reporting of behavior change interventions. Ann Behav Med. 2013; 46: 81-95.

14. Kartalova-O'Doherty $\mathrm{Y}$, Morgan K, Willetts A, Williamson T. Dementia - what is truth? Exploring the real experience of people living with more severe dementia. Mental Health Foundation. 2014.

15. McElveen T. Lying to people with dementia: treacherous act or beneficial therapy? Royal College of Psychiatrists, e-newsletter (September). 2015.

16. James IA, Caiazza. Untruths in dementia care: ethical decision making process. Signpost: J Dement Mental Health Care of Older People. 2016; 22: 6-9.

17. Pini S, Ingleson E, Megson M, Clare L, Wright P, Oyebode JR. A needs-led framework for understanding the impact of caring for a family member with dementia. Gerontologist. 2018; 58: e68-e77.

18. Edwards P. Lies, damned lies and dementia. J Dement Care. 2008; $16: 14$.

19. Wood-Mitchell A, Waterworth A, Stephenson M, James I. Lying to people with dementia: Sparking the debate. J Dement Care. 2006; 14: 30-31.

20. Caiazza R, James IA. Re-defining the notion of the therapeutic lie: Person-centred lying. FPOP Bulletin: 2015. 2015.

21. Woods B, Pratt R. Awareness in dementia: Ethical and legal issues in relation to people with dementia. Aging Ment Health. 2005; 9: 423-429.

22. James IA. The use of CBT in dementia care: $A$ rationale for Communication and Interaction Therapy (CAIT) and therapeutic lies. Cogn Behav Therapist. 2015; 8: e10.

23. James IA, Caiazza R. Therapeutic lies in dementia care: Should psychologists teach others to be person-centred liars? Behav Cogn Psychother. 2018; 46: 454-462.

24. Wilkinson $\mathrm{H}$. The perspectives of people with dementia: Research methods and motivations. Jessica Kingsley Publishers. 2001.

25. Day AM, James IA, Meyer TD, Lee DR. Do people with dementia find lies and deception in dementia care acceptable? Aging Ment Health. 2011; 15: 822-829.

26. Augen J. Bioinformatics in the post-genomic era: Genome, transcriptome, proteome, and information-based medicine. Addison-Wesley Professional. 2004. 


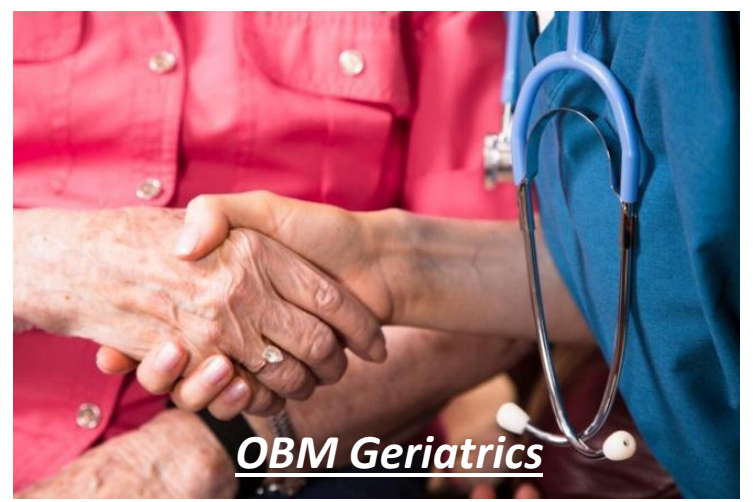

Enjoy OBM Geriatrics by:

1. Submitting a manuscript

2. Joining in volunteer reviewer bank

3. Joining Editorial Board

4. Guest editing a special issue

For more details, please visit: http://www.lidsen.com/journals/geriatrics 


\title{
Editorial
}

\section{Telomere Editorial - Perspectives on Telomeres and Aging}

\author{
Michael Fossel $^{*}$
}

Telocyte LLC, 250 Monroe NE, Grand Rapids, MI, USA; Michael.fossel@telocyte.com

* Correspondence: Michael Fossel; E-Mail: Michael.fossel@telocyte.com

Special Issue: Perspectives on Telomeres and Aging

\section{OBM Geriatrics}

2019, volume 3, issue 1

doi:10.21926/obm.geriatr.1901031
Received: January 29, 2019

Accepted: January 30, 2019

Published: January 31, 2019

The lack of research on the cause of aging is no less than a scandal because it is ignored by the medical establishment who ironically argue that aging is the greatest risk factor for their favorite disease.

Beginning in the 1960's [1, 2], cell senescence was first suggested as a central facet in aging, and therefore in age-related disease. In the early 1990's [3-5], key papers suggested that cell senescence was controlled by telomere shortening and that this correlated with age-related disease [6]. This was shown to be not merely a correlational, but a causal relationship in the landmark paper of 1998 [7], which showed that resetting telomere lengths could reset cell aging. This was followed thereafter by a series of papers [8-10] showing that this could be extended to in vitro demonstration of regrowth of young tissue from old cells or prolonged maintenance of young cell characteristics in skin, bone, and vascular tissue.

The first publications outlining the clinical potential of this field appeared in late 1990-2000 [1114], followed by the only textbook on this area [15], which described not only the genetic and cellular processes involved in age-related disease, but reviewed age-related human pathologies tissue-by-tissue, explaining both the probable role of cell senescence and the potential for more effective clinical intervention. The overall theory has been reviewed since [16] and a more recent

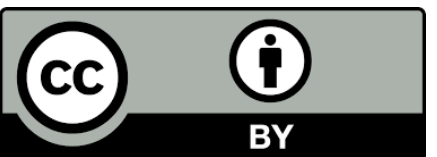

(C) 2019 by the author. This is an open access article distributed under the conditions of the Creative Commons by Attribution License, which permits unrestricted use, distribution, and reproduction in any medium or format, provided the original work is correctly cited. 
book [17] brought the field up-to-date, outlining the current status of the field and the practical prospects for human clinical trials.

In addition, a number of papers $[18,19]$ have demonstrated the success of using telomerase therapy in animals, resulting in extended healthy lifespans and, perhaps more importantly, strong support for the belief that we may more effectively intervene in age-related human diseases. As a result of these papers and ones like them, FDA-sanctioned telomerase gene therapy trials are planned.

Despite this history and its clinical potential, the model is not widely understood, and misconceptions are common, even within the academic literature. The model suggests a complex cascade of causation, which might be simplified as seen in Figure 1. We might summarize this model as: 1) cells divide, 2) telomeres shorten, 3) epigenetic changes result, and 4) cells show subtle but pervasive detrimental effects in their function. Such dysfunctional changes in cell function are ubiquitous. As examples, the turnover rate of most protein pools (e.g., enzymes, structural proteins, etc.) slows down, resulting in a gradual increase in the percentage of denatured (i.e., ineffective) proteins. As one example, the four major families of DNA repair enzymes become less effective, resulting in slower and less effective DNA repair, with a consequent increase in the percentage of mutational changes at any one time. In the mitochondria, the major aerobic enzymes are likewise turned over more slowly, decreasing the efficiency of the mitochondria and increasing the ROS/ATP ratio. Moreover, lipid membrane molecules - as they become oxidized or otherwise denatured - are more slowly replaced, culminating in leakier and less effective membrane barriers, with the result that (for example) ROS, the majority of which are created in the mitochondria, are more likely to traverse the mitochondrial walls and result in damage to molecules within the cytosol and nucleus. This, coupled with slower turnover among scavenger proteins (e.g., SOD, catalase, etc.) and a lower rate of turnover of damaged molecules in general, results in a gradual increase in oxidative damage to the cell.

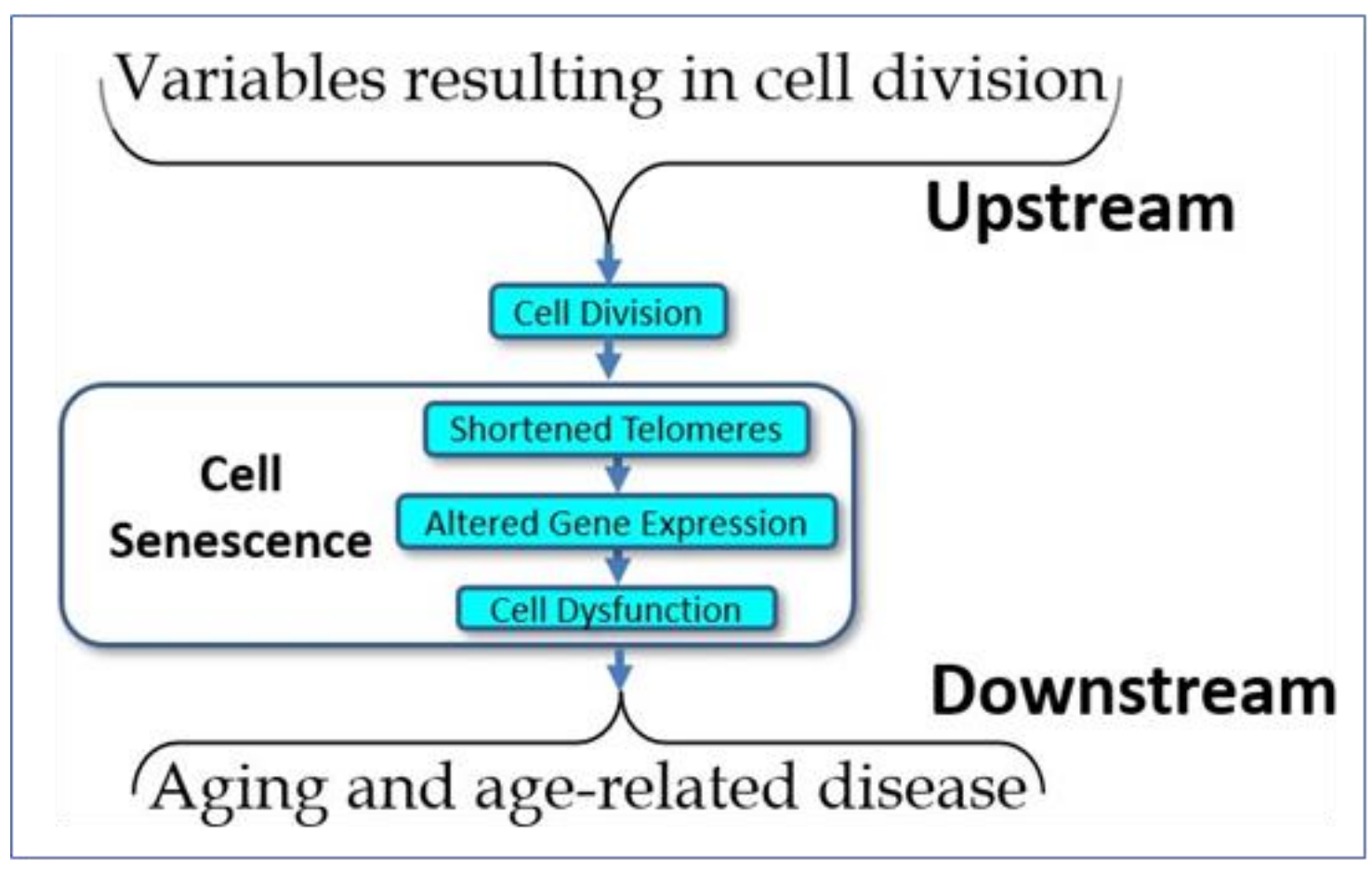

Figure 1 The simple model of telomere aging and disease. 
The upshot is that it is telomere shortening and - more importantly - the gradual changes in gene expression induced by such shortening - that results in cell aging and declining cell function results in a secondary loss of tissue function. Not only are intracellular molecules less likely to undergo turnover, but the same is true of extracellular molecules, such as collagen, elastin, amyloid, etc. The result of such slower extracellular molecular turnover not only results in such relatively harmless age-related changes as wrinkles (due to the slower turnover of collagen and elastin), but the tragic and disastrous changes of Alzheimer's disease (partially due to the slower turnover of amyloid proteins). In all cases, molecular pools within the body - whether intracellular or extracellular - are dynamic. Molecules are constantly being turned over and replaced. While different molecular pools undergo turnover at different rates, all molecular pools slow their rate of turnover with age, and the result is what we see as aging and age-related disease.

This model has not only proven consistent with all known data on aging and age-related disease, it has also proven predictively valid in trials of various interventions, such as those aimed at beta amyloid metabolism (e.g., Solanezumab, Aducanumab, etc.) mitochondrial function, or other interventional trials in the case of Alzheimer's disease. Finally, this model has not only withstood the tests of time but is eminently testable. When tested in animals (as cited above), interventions aimed at resetting gene expression - specifically, using telomerase expression - have been consistently shown to be effective in addressing age-related changes.

Several cautions remain. The first is that one common misunderstanding of the model is to assume that cell aging is a matter of telomere length, rather than relative telomere length. The critical variable is not telomere length but change in length. While some mice have telomeres that are 10x those of most humans, their lifespans are approximately one fortieth of most humans. This is entirely consistent with the model, which predicts that cell aging is a product of relative telomere loss, not absolute telomere length. More importantly, the key feature is the alteration that occurs in gene expression - epigenetic change - which is driven by telomere loss.

A second caution is the all-too-common use of LTL's (lymphocyte telomere lengths) as a valid measure of organismal aging, which is questionable, if not unwarranted $[20,21]$. While lymphocytic telomere loss is correlated with age, there are several problems with using LTL's as a dependent variable. The first problem should be obvious: lymphocytes are only one cell type among many and are scarcely the best indicator of what is happening in other cells, tissues, and organs. If, for example, we wish to get an indication of the risk of age-related vascular disease, the valid measure would be the telomere lengths of vascular endothelial cells, not the telomere lengths of lymphocytes. LTL's reflect the telomere lengths of such endothelial cells only roughly, in the sense that all somatic cells that divide are showing telomere loss correlating with age, and hence with one another. While true, humans die from specific age-related diseases and the status of one organ is not always an indication of the status of another. For example, your risk of heart attack may correlate with your risk of osteoarthritis (in that both correlate with chronologic age), but the best biomarker of heart attack risk is not the state of your chondrocytes, but the state of your arterial endothelial cells. A second problem with LTL's is that circulating lymphocytes represent only a small percent of the body's lymphocyte population, most of which are not in circulation. Worse yet, and depending on lymphocyte type (e.g., B cell, T cell, etc.) the major part of the loss of telomere length may vary in location from marrow to circulation depending upon stress to the organism. In practice, this means that there may be only minimal difference between the telomere lengths of the hematopoetic stem cells and circulating lymphocytes when there is 
little or no stress to the immune system, but a great deal of difference between stem cells and circulating lymphocytes when the immune system is stressed. The practical upshot is that LTL's do not provide an accurate indication of the "age" of the hematopoetic system except transiently when peripheral turnover is the exception.

Putting it simplistically, the telomere lengths of hematopoetic stem cells (which are rarely measured) show a very gradual and predictable decline with age, while peripheral lymphocytes are more likely to show a variable and unpredictable change over time, depending upon the needs of and the environment of the organism (see Figure 2). As LTL's may (and do) vary due to multiple factors, they are neither valid nor reliable indicators of the physiological age of the organism. They may be useful, but only when interpreted with great caution and then only in context. A final problem is that cell function (and the increasing dysfunction of senescing cells) correlates well with the minimal, rather than the mean telomere length, yet most studies (even when they identify their methods clearly) rely upon the cheaper, but less relevant mean telomere length, thus invalidating their conclusions.

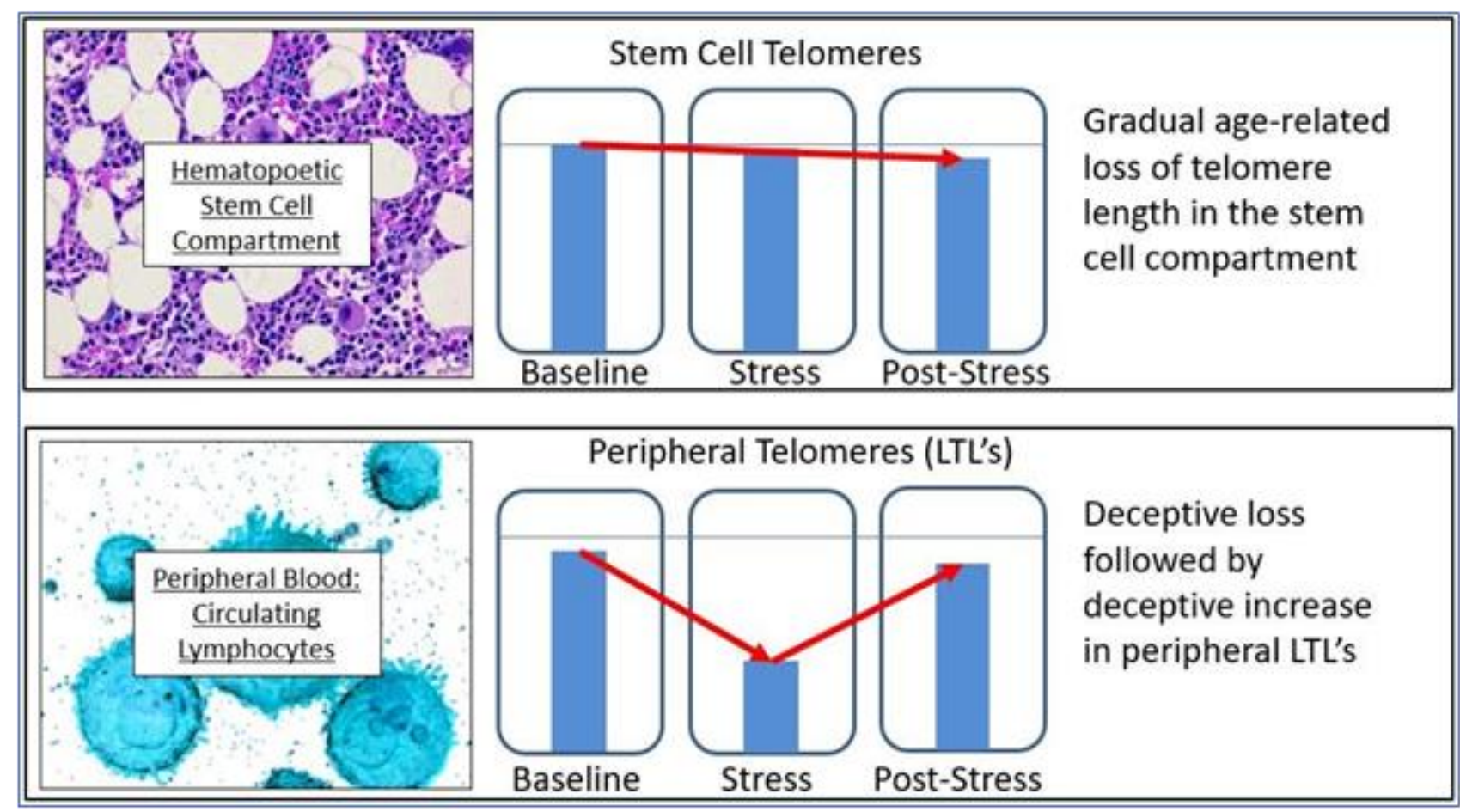

Figure 2 The problem with using LTL's.

The upshot of these several issues is that most current studies that purport to evaluate telomere lengths and assess potential variables or interventions, measure an invalid and unreliable variable and do so in an invalid and unreliable manner. While the conclusions of such studies may (perchance) be correct, the data used in these studies is insufficient support for the stated conclusions. As examples, the use of dietary improvements, exercise programs, meditation, or medication have all been suggested as potentially able to elongate telomeres (and improve health via this mechanism), but there is no adequate data supporting any of these conclusions.

The current state of the field - the importance to telomeres and telomerase in aging and agerelated disease - is confused and open to question, but the potential remains as enormous as it was suggested to be 20 years ago. Given what we do know about cell senescence, telomeres, and 
age-related disease, we might list both the obvious variables that affect cell aging ("upstream") and the dependent variables that result from cell aging ("downstream") (Figure 3). What is immediately apparent is that in almost all cases, current therapeutic approaches (and experimental trials) are targeted at downstream biomarkers, rather than targeting upstream variables or telomeres themselves. As all of the upstream variables affect telomere length (and gene expression) and as we are now capable of elongating telomeres in vivo, the single most effective point of intervention is not the dozens of upstream variables and certainly not the downstream biomarkers, but telomere length per se. Attempts to treat age-related diseases by addressing downstream biomarkers - such as targeting amyloid, tau proteins, mitochondria, or inflammation in the case of Alzheimer's disease - have not only proven uniformly ineffective, but we can now see why these approaches have been (and will continue to be) ineffective. Such approaches are essentially treating symptoms rather than treating the disease itself.

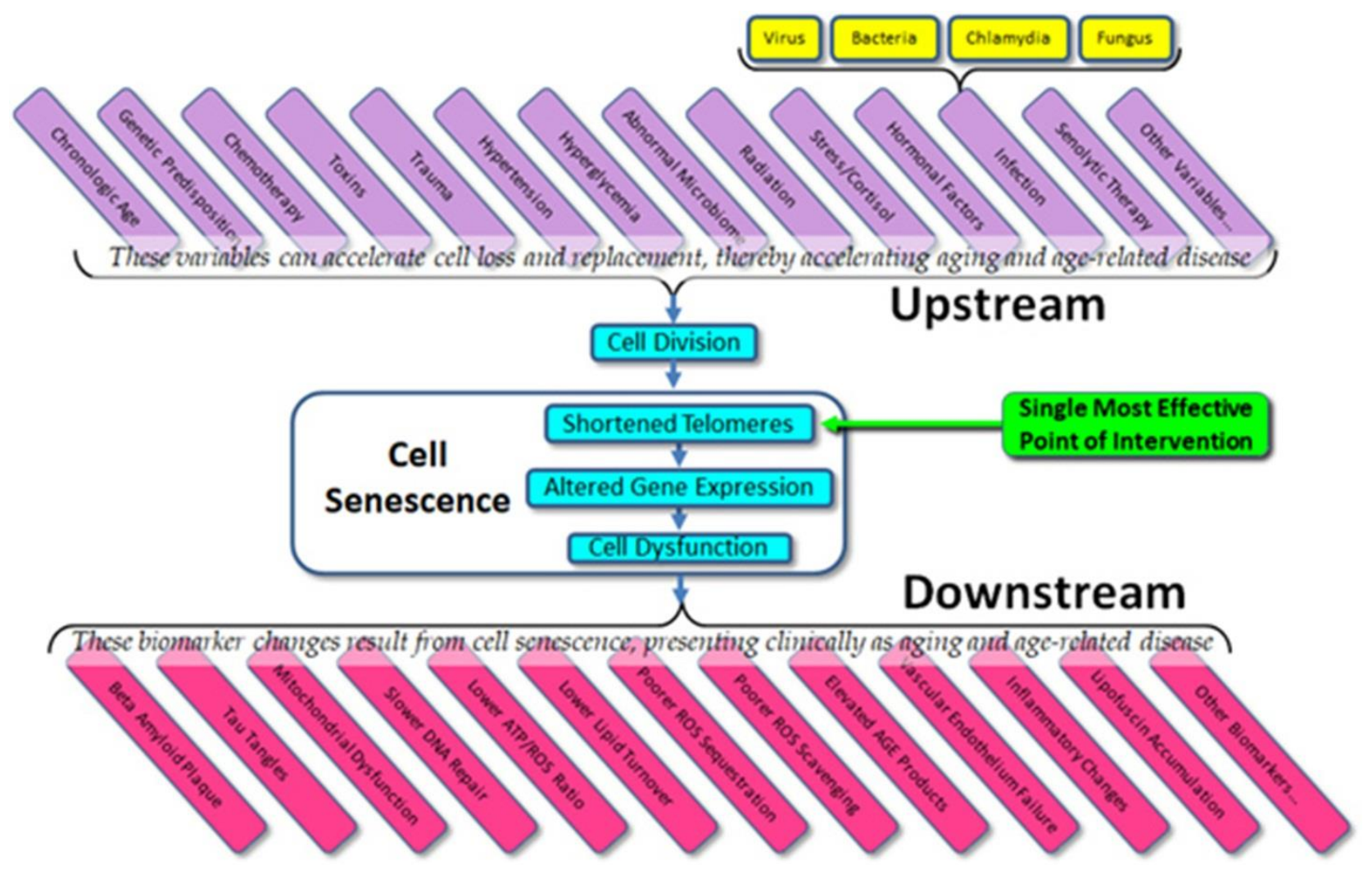

Figure 3 Upstream variables and downstream biomarkers in aging and disease.

Approaches that do not target telomere length, such as ablation of senescing cells will likewise prove futile, if not actually damaging (see related article in this issue). In cases in which we have targeted telomere length directly, not only do we find reliable improvement in all measured downstream biomarkers as well as general organismal health (and lifespan), but despite early concerns in the literature, there has been no evidence of cancer or other adverse effects $[19,22]$.

Understanding the role of cell senescence, telomeres, and telomerase in aging and age-related disease remains the most promising avenue for therapeutic intervention. The clinical potential of telomerase gene therapy is without any equal in current therapeutic approaches. 


\section{Author Contributions}

The author wrote this paper.

\section{Competing Interests}

The author has declared that no competing interests exist.

\section{References}

1. Hayflick L, Moorhead PS. The serial cultivation of human diploid strains. Exp Cell Res. 1961; 25: 585-621.

2. Hayflick L. The limited in vitro lifetime of human diploid cell strains. Exp Cell Res. 1965; 37 : 614-636.

3. Harley $\mathrm{CB}$, Futcher $\mathrm{AB}$, Greider $\mathrm{CW}$. Telomeres shorten during aging of human fibroblasts. Nature. 1990; 345: 458-460.

4. Allsopp RC, Vaziri H, Patterson C, Goldstein S, Younglai EV, Futcher AB, et al. Telomere length predicts replicative capacity of human fibroblasts. Proc Natl Acad Sci USA. 1992; 89: 1011410118.

5. Allsopp RC, Chang E, Kashefi Aazam M, Rogaev El, Piatyszek MA, Shay JW, et al. Telomere shortening is associated with cell division in vitro and in vivo. Exp Cell Res. 1995; 220: 194200.

6. Chang E, Harley CB. Telomere length and replicative aging in human vascular tissues. Proc Nat Acad Sci USA. 1995; 92: 1190-11194.

7. Bodnar AG, Chiu C, Frolkis M, Harley CB, Holt SE, Lichtsteiner S, et al. Extension of life-span by introduction of telomerase into normal human cells. Science. 1998; 279: 349-352.

8. Funk WD, Wang CK, Shelton DN, Harley CB, Pagon GD, Hoeffler WK. Telomerase expression restores dermal integrity to in vitro-aged fibroblasts in a reconstituted skin model. Exp Cell Res. 2000; 258: 270-278.

9. Yudoh K, Matsuno H, Nakazawa F, Katayama R, Kimura T. Reconstituting telomerase activity using the telomerase catalytic subunit prevents the telomere shortening and replicative senescence in human osteoblasts. J Bone Miner Res. 2001; 16: 1453-1464.

10. Matsushita H, Chang E, Glassford AJ, Cooke JP, Chiu CP, Tsao P. eNOS activity is reduces in senescent human endothelial cells. Preservation by hTERT immortalization. Circ Res. 2001; 89: 793-798.

11. Fossel M. Reversing Human Aging. New York, William Morrow \& Co. 1996.

12. Banks DA, Fossel M. Telomeres, cancer, and aging; Altering the human lifespan. JAMA. 1997; 278: 1345-1348.

13. Fossel M. Telomerase and the aging cell; Implications for human health. JAMA. 1998; 279 : 1732-1735.

14. Fossel M. Cell senescence and human aging: A review of the theory. In Vivo. 2000; 14: 29-34.

15. Fossel M. Cells, Aging, and Human Disease. New York: Oxford University Press; 2004.

16. Fossel M. Cell senescence in human aging and disease. Annals NY Acad Sci. 2006; 959: 14-23.

17. Fossel M. The Telomerase Revolution. Dallas: BenBella Books; 2015.

18. Jaskelioff M, Muller FL, Paik JH, Thomas E, Jiang S, Adams A, et al. Telomerase reactivation reverses tissue degeneration in aged telomerase deficient mice. Nature. 2011; 469: 102-106. 
19. de Jesus BB, Vera E, Schneeberger K, Tejera AM, Ayuso E, Bosch F, et al. Telomerase gene therapy in adult and old mice delays aging and increases longevity without increasing cancer. EMBO Mol Med. 2012; 4: 691-704.

20. Fossel M. Use of telomere length as a biomarker for aging and age-related disease. Curr Tran Geriatr Gerontol Rep. 2012; 1: 121-127.

21. Mensa E, Latini S, Ramini D, Storci G, Bonafe M, Olivieri F. The telomere world and aging: Analytical challenges and future perspectives. Ageing Res Rev. 2019; 50: 27-42.

22. Muñoz-Lorente MA, Martinez P, Tejera A, Whittemore K, Moises-Silva AC, Bosch F, et al. AAV9mediated telomerase activation does not accelerate tumorigenesis in the context of oncogenic K-Ras-induced lung cancer. PLoS Genet. 2018; 14: e1007562.

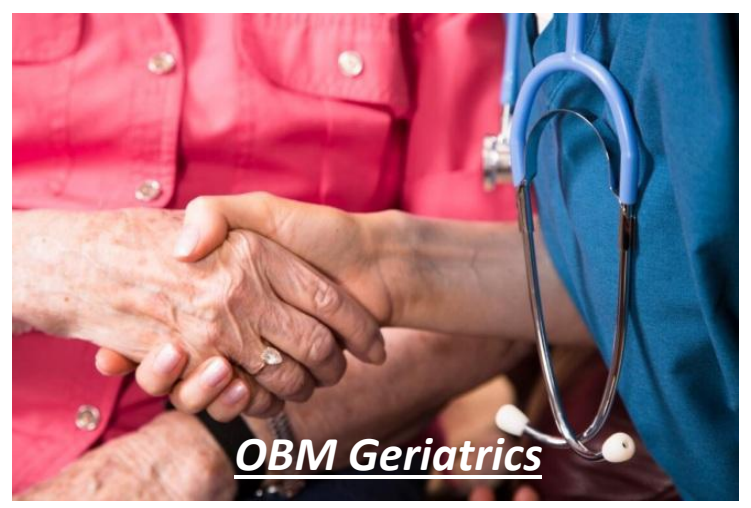

Enjoy OBM Geriatrics by:

1. Submitting a manuscript

2. Joining in volunteer reviewer bank

3. Joining Editorial Board

4. Guest editing a special issue

For more details, please visit: http://www.lidsen.com/journals/geriatrics 
Research Article

\title{
An Examination of How the 'Household Model' of Care Can Contribute to Positive Ageing for Residents in the 'Fourth Age'
}

\author{
Anya Ahmed *, Paula Ormandy, Maaike L. Seekles
}

School of Health and Society, University of Salford, Manchester, United Kingdom; E-Mails: a.ahmed@salford.ac.uk, p.ormandy@salford.ac.uk,m.l.seekles1@salford.ac.uk

* Correspondence: Anya Ahmed; E-Mail: a.ahmed@salford.ac.uk

Academic Editor: Lisa Hollis-Sawyer

Special Issue: Got Aging? Examining Later-life Development from a Positive Aging Perspective

\section{OBM Geriatrics}

2019, volume 3, issue 1

doi:10.21926/obm.geriatr.1901030
Received: November 15, 2018

Accepted: January 21, 2019

Published: January 25, 2019

\begin{abstract}
Background: Promoting a good quality of life for the oldest members of society has become a top priority as evidenced in UK policy. The 'household' model is a departure from traditional approaches to care provision since it offers person-centred support - combing health and social care - to older people in specially-designed, small, homelike environments. Having gained increasing popularity in care homes across developed countries, the impact of this model of service delivery on residents' quality of life and its contribution to positive ageing is of increasing interest. Belong is a not-for-profit, UK care organisation currently operating several villages under the household model. The villages comprise independent living apartments (bought or rented) and residential/nursing care households offering 24 hours personalised, on-site care for residents. In each village there is a range of facilities open to the public (including a Bistro, hairdressers and gym facilities) and a domiciliary community service.
\end{abstract}

Methods: In this paper we present new data generated from qualitative interviews with a sample of household residents in the 'Fourth Age' and relatives across two villages in the North West of England, UK.

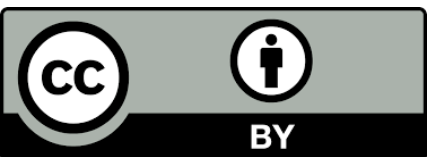

(C) 2019 by the author. This is an open access article distributed under the conditions of the Creative Commons by Attribution License, which permits unrestricted use, distribution, and reproduction in any medium or format, provided the original work is correctly cited. 
Results: We examine how the household model as operated at Belong facilitates the maintenance of autonomy and independence -which underpins positive ageing and quality of life in the fourth age - among residents.

Conclusion: We show how the household model contributes to positive ageing and quality of life as defined by residents.

\section{Keywords}

Positive ageing; Fourth Age; household model; care home; Belong; UK

\section{Introduction}

Over the last half century, the UK has undergone significant structural change in that the number of people living into very old age has risen quite remarkably [1]. This demographic shift is largely due to medical advances impacting on life expectancy and, as a result, the number of people in England aged 60 years and over is predicted to rise by almost 33\% between 2014 and 2030 [2]. Within this cohort, the number of very elderly people aged 85 and above is projected to increase at the fastest rate, rising by $60 \%$ [2].

A division of later life has since been recognised which challenges the assumption that just one "old age" exists [3], and, instead of old age representing an entirely chronological phenomenon, it is often thought of in terms of two distinct phases; the third and fourth age [4].

The third age - or "young old age" - typically represents the post-retirement period when older people, who at this stage have lived out their career and family-rearing responsibilities, can exercise greater agency and choice and are faced with opportunities for self-fulfilment and purposeful engagement [5]. This age is generally characterised by good health and physical functioning, attributions of wisdom, social participation, and adaptive flexibility in daily living [6-9]. Contrastingly, it is the loss of agency and choice which differentiates the fourth age from the third age $[7,10]$. Whilst there is no definitive chronological onset, the term "fourth age" - or "old old/deep old age" [3, 7] - denotes those people in the very later years of life, the "oldest-old", from approximately 85 onwards [7]. More defining of the fourth age is the associated physical, cognitive and psychosocial decline - what Baltes [11] referred to as the "negative biological trajectory of the life course" (p. 368). This is associated with the onset of bodily decline and a loss of independence and mobility [7] and an increased dependence on formal care services including long-term care in residential or nursing homes [12]. However, there has recently been a recalibration of how ageing is constructed and perceived by wider society in gerontological research and among policy circles and this has had significant impact on the models and ethos of care home provision. The contemporary focus is on 'ageing well' and positive ageing which represents a new paradigm in gerontological research and a departure from more negative aspects of ageing [13].

Over the past two decades in the UK there has been continued policy emphasis on personalised care and support for older people whereby service users exercise their right to choice and control. In this way, older people are no longer perceived as passive recipients of care, but rather as active agents. This has also meant that service providers are expected to encourage people to maintain 
autonomy and independence in order to promote a good quality of life whilst simultaneously offloading pressure from public funding streams [14].

\subsection{Aims of Paper}

This paper is drawn from research undertaken as part of an ongoing wider Knowledge Exchange project between The University of Salford and Belong Ltd. which took place between February 2017 and January 2019. The wider project aims to identify how Belong - which operates a household model of care - impacts on residents' quality of life, staff, and the wider community to identify and develop ways of measuring and monitoring this over time. This paper aims to examine how living in a household in Belong contributes to positive ageing for residents in the fourth age. Here we take account of what positive ageing and a continuation of quality of life mean to residents themselves. The paper is structured in the following way: first, we introduce Belong; then ageing in conceptual, theoretical and policy contexts is discussed; third, the culture change movement in the care home sector is outlined to further contextualise the Belong model; fourth, the methodology and methods underpinning the paper are presented; fifth, the findings from the interviews with residents and relatives are analysed and discussed; finally, we offer a conclusion, stating our contribution to knowledge.

\subsection{The Belong Model}

Belong is a values-driven, not-for-profit care organisation, with the aim of creating community 'villages' which provide a range of accommodation and support services to people living in the village and in the wider community. The Belong brand was launched in 2007 and since then, seven villages have opened across the North West of England, with two more set to open in 2019 and 2020. The villages are comprised of apartments available for rent or sale, attached to a care home facility in which care is provided in 'households'; typically, six households are included in each village. The vision underpinning Belong's model of service delivery is one of 'providing a home for life, where older people have the right to enjoy the same community belonging and quality of life that they have always known' [15].

Guided by their vision, Belong has set the following commitments to its customers:

- Belonging to a vibrant community

- Enjoying a home for life

- Living an active lifestyle

- Offering choices and independence

- Nurturing relationships

- $\quad$ Giving peace of mind [16]

These commitments are translated into practice in several ways. First, within the main building of the villages, a community environment is created through the presence of a Bistro, hairdressing salon, therapy room, exercise studio -with personalised exercise programmes-, internet café and venue for hire. These facilities are all open to the public. Residents and members of the community can also access a programme of events and activities inside or outside of Belong, with opportunities for interaction with animals, children and the outdoors. Also, as a different approach to specialist day care, Belong organises so-called 'Experience Days', for people from outside of Belong who would like to be supported in their use of the facilities. During such a day, a 
member of staff helps customers to co-ordinate their day to enjoy meaningful activities and be part of the village community. In addition, Belong offers a variety of services which enable users to experience holistic support as their needs change. These range from: independent living in Belong apartments, to domiciliary care for people in the wider community, through to residential or nursing care within the 'households' in the home, which includes end-of-life support.

The households are small-scale, dementia-friendly domestic environments in which 24-hour health and social care is provided to residents. The small-scale domestic settings which characterise Belong reduce the amount of potentially overwhelming stimuli and risk of disorientation which could negatively impact on people with dementia. The ethos underpinning the design is the importance of promoting stability and familiarity with surroundings. Households contain a mix of residents, with people living with dementia, or nursing care needs, living together with those who do not. 71.8 per cent of all household residents in Belong have a diagnosis of dementia and staff are trained in dementia awareness and an Admiral Nurse ${ }^{1}$ works across the village to specifically support residents with dementia and their relatives. The model recognises that needs often fluctuate and accordingly, care can be tailored to residents as required. Each household has a dedicated support team which always works with the same residents, enabling staff to get to know them well.

The households are designed to be an 'extended family sized community' for 10 to 12 residents, with ensuite bedrooms that lead directly to an open-plan, shared communal space. At the heart of each household is a kitchen, which is central to generating shared experiences between residents and staff. In the mornings, staff often bake a cake in the kitchen and throughout the day, a host helps to deliver 'marvellous mealtimes'. As part of 'marvellous mealtimes', residents are offered menu options, and can assist with meal preparation or table setting as they wish. Meals are prepared and cooked in the household kitchen to simulate the sight, smell and sounds of cooking which are commonly associated with home. While mealtimes as such are flexible around a resident's preferred schedule - and residents are free to decide when and where they would like to eat - Belong aims to promote a family mealtime atmosphere to encourage and facilitate interaction between residents and staff. Residents, relatives and friends are free to help themselves and others to refreshments throughout the day.

Many care homes operate a 'medical model' of care, which encourages a 'sick' definition of self in residents. Such residential facilities optimise structures of rigid routine, excessive 'risk' management and use of chemical restraints to reinforce passive or dependent behaviour in residents which is then more easily controlled by staff [17]. However, in Belong, this kind of institutionalised ethos is specifically avoided. Indeed, in Belong, residents need not adhere to a specific waking routine and there are no tea trolleys, bathing or medication rounds, as residents or 'customers', as they are known - are supported holistically in a place and at a time they choose. The use of anti-psychotic medication and sedatives is kept to an absolute minimum for people with complex needs related to dementia; instead, staff take time to observe and address causes of distress. In addition, Belong's vocabulary avoids the use of such terms as units, patients, and 'sufferers', instead encouraging the use of positive language around households (rather than

\footnotetext{
${ }^{1}$ Admiral Nurses are specialist dementia nurses who give expert practical, clinical and emotional support to families living with dementia
} 
residential care units), Experience Days (for day care) and independent apartment living (not extra care).

Exercise is high on the agenda at Belong. A dedicated gym instructor, with both care and fitness experience, aims to create a personalised exercise plan for each household resident to improve mobility and wellbeing. The instructor will either support residents in the exercise studio, or in the household. In addition, chair-based exercise classes are offered. Belong also has a large garden area and residents are encouraged and supported to use this.

The staff to resident ratio in Belong is higher than average, with three staff per household and a floating nurse on shift, which allows staff to spend more time with residents. In the UK, minimum hours of care or ratios of care are not specified in national regulations. However, the UK Royal College of Nursing published guidelines for nursing homes with a patient to staff to resident ratio of 1:5 for early shifts, 1:6 for late shifts, and 1:10 for nights (with an average of $35 \%$ registered nurses and $65 \%$ care assistants). Belong aim for a ratio of 1:4 staff to residents in the households between $8 \mathrm{am}$ and $10 \mathrm{pm}$ plus at least one nurse on duty $24 / 7$. There is at least one nurse on duty $24 / 7$ who is responsible for directing the care of residents who have nursing needs. One of the senior team is on call out of hours. During the night, there is a dedicated waking support worker per household, in addition to a floating support worker and the floating nurse.

Staff work closely together with primary care teams and hospitals, in order to keep hospital admissions and stays to a minimum. Wherever possible, people are cared for at home in Belong, right up until the end of their lives. Staff are provided with learning and development opportunities as Belong aims for all staff to hold an accredited qualification. In addition, a Practice Development Facilitator works across each village to ensure that staff have the skills, confidence and support to work effectively, have undertaken core training and to identify any additional training needs. In April 2017, Belong chose to substantially increase the pay for all hourly paid staff on the National Living Wage (NLW) with $17.4 \%$. Those members of staff are now remunerated in line with the Living Wage, which is an hourly rate that is calculated according to the basic cost of living in the UK. This increase has meant that a care worker in Belong is now paid more than the average care worker in the UK, according to data from the National Minimum Dataset for Social Care as presented by Skills for Care.

Belong's approach to care and management of its staff has been recognised as a good practice by several organisations providing relevant performance and quality standards and all Belong villages have received a Care Quality Commission (CQC) rating of 'good' or 'outstanding'. The CQC, as a regulator, investigates whether the services provided are safe, effective, caring, responsive to people's needs, and well-led. In addition, Belong is part of the National Gold Standards Framework (GSF) in Care Homes Programme for palliative care. This programme aims to improve the quality of care, inter-agency collaboration, and support for individuals who wish to die at home, for example by promoting choice and control at end-of-life through advance care planning. Most of the existing villages have received GSF accreditation, while the newer villages are working towards achieving it. Belong was awarded the international award of 'Excellence in the Third Sector' of Investors in People in 2018 for its investment in 'class-leading recruitment, training and development opportunities'. Finally, Belong received the Investors in People Gold Standard accreditation in 2016 [18]. 


\subsection{Ageing in Conceptual, Theoretical and Policy Contexts}

'Ageing well' is the lay term for a positive trajectory of ageing, encompassing labels such as healthy, successful, competent, optimal, vital, active, productive (and positive) ageing. These terms can be understood as constituting a 'semantic network' representing a shift to a positive paradigm in gerontology research [19]. Healthy and successful ageing are used most commonly in biomedical research, with healthy ageing denoting an absence of illness and functional independence, while successful ageing integrates additional biomedical and psychosocial conditions including a low probability of illness and disability, cognitive fitness, positive effect and control and social participation [19]. However, a purely biomedical approach to successful ageing neglects the psychological and social complexities associated with growing older [20], since it does not take account of older people's perceptions of what constitutes successful ageing for themselves [21]. For example, previous research has shown that many people subjectively rate themselves as "successful agers" despite not conforming to the objective "disability/illness-free" definition [22]. Often, older adults perceive successful ageing more in terms of psychological and emotional wellbeing, activity participation, enjoyment of leisure and experience shared with others, than in terms of their physical health [23]. By this definition, successful ageing is more commonly associated with the third age which then implies that those in the fourth age are "unsuccessful". Historically, adopting this approach has led to models of care predominantly focused on the treatment and management of physiological ailments [24]. Such a stance led to a social construction of ageing as a "medical problem" encouraging the view that ageing is abnormal [24]. Applying this 'problem' of ageing to long-term care provision historically raised the misconception that older people are passive recipients of care practices "based on hygiene, pressure area care, medications and food" (p. 955) [25].

There are issues in operationalising concepts of ageing, there are different meanings associated with different terms [19] and we do not treat these as being synonymous. Although the focus of this paper (and the special issue) is on positive ageing, active ageing principles underpin the policy framework which shapes care home provision (and therefore the Belong model, also influenced by the culture change in the care home movement). Therefore, there are some relevant overlaps and we identify these below, particularly in relation to how both terms are concerned with quality of life. Autonomy and independence are recognised as factors that are associated with active ageing, an approach which challenges the passive and dependent stereotypes commonly synonymous with older age [21]. Defined as "the process of optimising opportunities for health, participation and security in order to enhance quality of life as people age [26], active ageing is regarded as a mechanism through which to address the challenges of population ageing [21]. The responsibility of active ageing lies both at a societal (structural) level in that policy action should afford empowerment and opportunity for activity amongst older people, and at an individual level (agency) with older people exercising their personal freedom to engage with these opportunities [21]. Potential outcomes for the individual include social inclusion, enhanced wellbeing and quality of life, and at a societal level, reduced pressures on public spending on pensions, health and social care $[21,27]$. According to the World Health Organisation, which introduced the term [26]:

"Active ageing policies and programmes are needed to enable people to continue to work according to their capacities and preferences as they grow older, and to prevent or delay disabilities and chronic diseases that are costly to individuals, families and the health care 
system... People who remain healthy as they age face fewer impediments to continued work... This would help to offset the rising costs in pensions and income security schemes as well as those related to medical and social care costs." (p. 9).

In practice, however, active ageing policy across Europe has been criticised for focusing too narrowly on labour productivity, which excludes and potentially stigmatises those who are unable to work, in particular the oldest-old in the fourth age [28]. Active ageing then, should not be entirely focused on physical activity and participation in the labour market but should be also be inclusive of social, cultural, spiritual and civic affairs [26]. Such "activity" should be meaningful and contribute positively to the wellbeing of older people and that it should be inclusive of all older people, including frail and dependent older people in the fourth age [29]. The goal of active ageing in the fourth age therefore, should be on maximising participation and autonomy. In this sense, a broader approach to active ageing supports the notion that it maintains relevance throughout the ageing process as a whole, as opposed to being applicable only to those in the third age [30]. A commonality between positive, active and successful ageing is that they emerged as opposing responses to disengagement perspectives of ageing [29]. The largely discredited body of disengagement theories of ageing propose that as people experience the transition from the third to fourth age, they experience a lessening of social interactions and an inevitable withdrawal from society occurs [31,32]. However, these assumptions are incompatible with empirical evidence which shows many older people continue to engage with employment, political and social domains of life in particular [33].

It is also important to take account of older people's interpretations and expectations of 'ageing well' in their own lives [28]. A previous study of active ageing in a care home context [30] found that key determinants were reported as: a welcoming, homelike environment and culture; having choice and control over decisions and care; the opportunity to participate in meaningful activity; a sense of usefulness; and the promotion of independence, neighbourhood integration and social participation. Thus, despite a physical and mental decline in this age, it seems clear that active ageing can both useful and applicable to people in the fourth age who are living in a care home context [30] and that there is a role for care provision in facilitating this. Those elements related to the maintenance of quality of life encompassing positive emotional states and social integration (included in definitions of positive ageing) are key to our analysis.

Multidimensional concepts of positive ageing encompassing 'objective indicators' (including fitness/ health and optimal cognitive functioning) and 'subjective indicators' (including positive emotional states and social investment) have been proposed and these have methodological implications [13]. In this paper, our analysis is undergirded by an interpretive epistemology, based on residents' subjective accounts of their own experiences of positive ageing, as the importance of understanding such experiences from a residents' perspective are key (42). In this way, we focus on residents' recounting of such 'subjective indicators' within the context of health and cognitive function in the fourth age, rather than focusing on biomedical factors per se, in part since in this stage of life the applicability of the objective indicators reduces [13]. The fourth age is not a continuation of the third age since it is largely characterised by pathology - health status and functionality - so healthy and successful ageing has limits from a biomedical perspective when understanding people's experiences of positive ageing in the fourth age (Baltes and Smith, 2003 cited in Fernandez- Ballesteros et al, 2013 [19]). 
Thus, we apply positive ageing as a conceptual framework, yet we conceptually engage with active ageing as the policy framework though which positive ageing is delivered. In this way active ageing can be understood as the policy mechanism through which to address the challenges of ageing. Our focus is on subjective indicators (positive emotional states and social investment) of positive ageing. In other words, this is not a focus on the number of illnesses or measures of cognitive and physical function instead we address residents' subjective interpretations of wellbeing (positive emotional state) and the maintenance of social investment (or social interaction). We focus on positive ageing and therefore how this can be facilitated into the fourth age in terms of such subjective indicators and how this is inextricably linked with quality of life and can continue in spite of physical and cognitive decline [13]. We posit that the household model as operated by Belong facilitates this since it focuses on people as active agents, rather than passive recipients of care, and the promotion of positive emotional states and social investment: representing continued quality of life [13].

Clearly then, quality of life is a broad and multifaceted concept encompassing physical, social and psychological aspects of life [34]. However, despite its complexity, the core element to quality of life is that it signifies what makes life meaningful, enjoyable and worth living as defined by the individual [35]. Any such commitment to promoting quality of life in care homes needs to be driven by what matters most to the individual and how they wish to be supported, as opposed to staff assumptions about what older people want [35].When moving to long-term care facilities, it is important for quality of life to be preserved in terms of 'feeling at home' for older people. Cooney [36] identifies four core features of finding a home, including: 'continuity', 'preserving personal identity', 'belonging' and 'being active and working'. Continuity in this respect relates to older people's continued engagement in their normal, everyday activities and maintenance of their usual routines, for example, waking and going to bed when they choose and dressing how they like. Preserving identity relates to older people having privacy, having their own personal space with their belongings around them, and feeling known and valued as an individual. Belonging is associated with feeling part of a group and experienced as a sense of solidarity, companionship, relaxation and fun. Finally, 'being active and working' relates to the opportunity to engage in both meaningful activities, as well as those that constitute daily activities such as socialising, watching television and domestic activities of daily living [36]. These findings are also consistent with the work of Nolan et al [37], who developed a "senses" framework intended to inform best practice in care home environments. The framework highlights that where a service affords good quality of care, users will experience a sense of security, belonging, continuity, purpose, achievement and significance.

Many of the discussed practices, behaviours and attitudes associated with a good quality of life - continuity, maintenance of personal identity, meaningful relationships, personalised care, autonomy, choice, control and involvement in decision-making, a positive living environment, safety, and meaningful daily and community life - feature as themes in Blood's [38] review relating to what makes for a 'better life' for older adults. The evidence suggests then, that models of care for older people should adopt a holistic approach considering health, well-being, physical, cognitive and social functioning, as well as continued engagement with social life and activities if they are to promote positive outcomes/experiences for older people: in other words, facilitate the maintenance of quality of life into the fourth age. 


\subsection{The Emergence of The Culture Change Movement: Towards The Household Model of Care Provision}

The recurring themes throughout the above literature and policy review: choice, empowerment, autonomy, independence, activity, identity, personalisation, relationships, and community, reflect how developments in research and policy have transformed the image of old age from one that is negative and fixated with illness and disease, to one that reflects positivity, wellbeing and quality of life. In doing so, research and policy initiatives have facilitated a culture change within care home settings, evolving innovative new models which diverge from traditional care home practice through the reconceptualisation of the structure, roles, and processes of care [39]. Having provided the policy context within which models of care are currently operating, we now contextualise the culture change movement and review a number of models to contextualise Belong.

As meeting the psychosocial needs of older people has become a priority in the context of long term care, it has become increasingly recognised among consumers, policy makers, and providers that the traditional, institutional model of care limits autonomy and is unconducive to this aim [40]. As such, throughout the past three decades innovative philosophical developments have emerged which have sought to transform institutionalisation towards care which puts older people at the forefront of all decisions and practices; a change which makes "long-term care less about care tasks and more about caring for people and the relationships between people"(p.xiii) [41].

As highlighted above, policy and legislative initiatives across developed countries that have mandated an individualised, person-centred approach to care and this has helped to inspire the grassroots culture change movement in care homes [42]. The birth of this movement is generally attributed to developments in the USA during the late 1990s when a group of care service providers, researchers and consumer advocates collaborated to determine the common principles that featured in their vision for culture change in care homes [43]. This collective group, known as the Pioneer Network, articulated thirteen values that characterise the underlying ethos of culture change which include: Know each person; Each person can and does make a difference; Relationship is the fundamental building block of a transformed culture; Respond to spirit, as well as mind and body; Risk taking is a normal part of life; Put person before task; All elders are entitled to self-determination wherever they live; Community is the antidote to institutionalization; Do unto others as you would have them do unto you; Promote the growth and development of all; Shape and use the potential of the environment in all its aspects: physical, organizational, psycho/social/spiritual; Practice self-examination, searching for new creativity and opportunities for doing better; Recognize that culture change and transformation are not destinations but a journey, always a work in progress [43]. The overarching goal of culture change is to offer homelike environments to older people in which maximum quality of life can be achieved for both residents and staff $[44,45]$.

To better illustrate the philosophies that underpin resident-directed practices inherent to culture change in care homes, it is now useful to review some of the contemporary models in operation. The Eden Alternative (EA), founded by Dr William Thomas, was one of the first culture change models in the early 1990s. Thomas rejected the medical models "fixation on diagnosis and treatment" believing this fosters care of low quality [46]. The EA model premised that residents 
should be treated with dignity and respect, valued as individuals and be the active decisionmakers when determining their daily lives. The EA model stipulates that care settings should be homelike, social and vibrant environments including the presence of plants, animals and children. Early evaluation studies of the EA approach returned conflicting results about improvements in physical health $[47,48]$. However, evidence suggests that resident and family satisfaction and residents' independence improved $[49,50]$.

The culture change movement in care homes is viewed as a method of continuous quality improvement [51]. Thus, with early research yielding conflicting findings relating to the benefits of an EA, it became clear that more systemic and structural changes within the structure of care homes were necessary $[52,53]$. Indeed, pioneers of the culture change movement recognise that in order to facilitate positive outcomes for older care home residents, radical physical reconfiguration of traditional care home environments is necessary to implement the principles underlying culture change [42]. A fundamental aspect within the vision of the culture change movement was that it should be more representative of a genuine "home" [54]. The fact that traditional facilities are large and unrepresentative of the average family home provided the impetus to de-scale to smaller "households" - hence, the "household model" was conceptualised.

The predominant characteristic of a care home operating a household model is that it comprised of smaller, homely units intended to house between 6-12 residents. The model has been described as "a living arrangement where all activities of daily living occur within a small scale environment, reminiscent of a large family home... an environment that is immediately understandable to residents and visitors as a setting that has been a natural part of everyday life" (p.9) [55]. The underlying philosophy of the household model is akin to that of the Eden Alternative, that service provision is customer focused and process oriented delivered by a consistent team of care workers, as opposed to a service-led, task-orientated approach operating in traditional cultures where older people are perceived as passive recipients of care $[54,56]$.

The first incarnation of the household model was in the USA in 1997 at Creekview in Evergreen Retirement Community, Wisconsin. A 'neighbourhood' of 36 residents was designed, made up of four small-scale households housing nine residents [54]. Each resident had their own private bedroom with en-suite facilities centred around an open plan, shared living, dining and kitchen area. Residents also had access to a secure garden, allowing continued exposure to the natural environment [55]. A household model of a similar structure operating in Australia, known as the ADARDS model, involves four small households of nine residents connected around a centralised room. The individual households operate independently during the day and cooperatively as one unit over the night allowing for lower staff-to-resident ratios during this quieter period [57]. Flexibility is central to the philosophy of the model, for both staff and residents [57], and staff can organise their own working rotas to accommodate family life [58]. Flexibility in residents' daily scheduling fosters an atmosphere conducive to individualised care and is intended to remove the regimented routines found in traditional institutions. Meal times are innovatively structured to create a shared and meaningful experience whereby staff members are encouraged to eat with residents and relatives, avoiding the social divide associated with the traditional care home model.

Additionally, the physical environment plays a significant role in shaping the actions of individuals and groups, by providing opportunities for the preservation of identity to be supported, as well as facilitating opportunities for social interaction through which positive relationships can be established [55]. Within traditional institutional settings preservation of identity is largely 
prohibited with shared bedrooms and bathrooms, offering limited space for residents' personal belongings. Care home environments that facilitate a sense of familiarity have the potential to offer plenty opportunity to engage with a range of activities and provide a comfortable balance of private and community spaces and are associated with a higher quality of life [59].

However to date, there is limited evidence of the impacts of the household model on residents $[60,61]$. In their systematic review, Ausserhofer et al. [60], explored the impact of homelike residential care models for residents with and without dementia on resident, family and staffrelated outcomes. They found only 14 studies, conducted between 1994 and 2014, with most studies examining behavioural or quality of life outcomes. The six studies on quality of life that were identified shared mixed findings, with some presenting improvements in some, but not all domains of quality of life; better outcomes compared to one nursing home but not the other; and/or better outcomes in some domains, but worse in others compared with traditional settings [60]. Of the studies reporting positive changes, the physical and psychosocial benefits of a household model approach to care are emphasised [62]. For example, Morgan-Brown, Newton and Ormerod [63] measured resident social and activity engagement in two Irish care homes preand post-implementation of a household design. The changes included adopting an open plan design with a unit kitchen, employing staff in a homemaker role and transforming task-based provision to person-centred care. Within both facilities, engagement with interactive occupation (defined as activity such as participating in a game or craft activity) and social interaction significantly increased following implementation of a household design [63]. Other reported positive benefits associated with small-scale living arrangements in include higher motor functioning and mobility; less anxiety, and depression [62].

\section{Materials and Methods}

\subsection{Methodology}

The methodology underpinning this paper is qualitative with commensurate methods: in-depth semi-structured, face-to-face interviews, and is congruent with our focus on exploring the subjective elements of positive ageing as explained above. A purposive sample of residents was taken to reflect a mix of gender, age, time lived in Belong and village location. Although we acknowledge that the sample is not necessarily representative of all residents in Belong, nor of older people in care home environments, we make an empirical and theoretical contribution to knowledge as outlined in the conclusion. A total of 18 interviews took place with 14 household residents $^{2}$ and three family members across two villages. A list of residents' names was obtained from management staff who were able to advise whether the resident would be able to undertake an interview. Contact details of spouses or family members were then obtained for those people who were unable to participate. Four of the residents' relatives were subsequently interviewed, three because the resident was living with dementia which impacted on their capacity to undertake the interview. The interviews took place in two Belong villages between September 2017 and October 2018.

\footnotetext{
2 'Nigel' was interviewed twice - once with his daughter in September 2017 and again on his own (both times at his request) in October 2018
} 
Participants were sent an invitation letter and information sheet via post and were asked to register their interest in being interviewed. Those who confirmed interest where then invited for an interview asked to provide consent on the day the interview took place. The interviews lasted for an hour on average and focused on: experiences of living in a Belong village; transitions to a care home environment; views on care, activities and facilities; being able to exercise control and autonomy; and interpretations and experiences of continued quality of life.

The characteristics of the residents can be found in Table 1. The average age of all participants was 85.5 years, ranging from 69 to 97 years. Most of the participants (15 out of 17) were currently living with one or more chronic long-term conditions, including, but not limited to, Dementia, Parkinson's Disease, Multiple Sclerosis, Chronic Obstructive Pulmonary Disease, Chronic Kidney Disease, Cardio-Vascular Disease, and various forms of cancer. In order to protect the identity of all individuals, pseudonyms are used and the location of the village where they live is omitted. The research was carried out to the standards set in the ESRC Research Ethics Framework and the British Sociological Society's Statement on Ethical Practice. In accordance with these guidelines, the research was conducted with the welfare of participants in mind and the overarching project received ethical approval through the University of Salford's Research Ethics Panel.

Table 1 Resident Profiles

$\begin{array}{llll}\text { Resident } & \text { Gender } & \begin{array}{l}\text { Resident's age at } \\ \text { time of interview }\end{array} & \begin{array}{l}\text { Time in Belong (at } \\ \text { time of interview) }\end{array} \\ \text { Arnold (interview with Christine, Arnold's spouse) } & \text { Male } & 74 & 41 \text { months } \\ \text { Elsie (interview with Ralph, Elsie's son) } & \text { Female } & 85 & 10 \text { months } \\ \text { Margot (interview with Don, Margot's spouse) } & \text { Female } & 79 & 53 \text { months } \\ \text { Nigel, interviewed with his daughter Sally } & \text { Male } & 86 & 30 \text { months } \\ \text { Sandra } & \text { Female } & 69 \text { (deceased } & 18 \text { months } \\ & & \text { since interview) } & \\ \text { Rose } & \text { Female } & \text { since interview) } & 5 \text { months } \\ \text { Norma } & \text { Female } & 97 & 10 \text { months } \\ \text { Alice } & \text { Female } & 80 & 8 \text { months } \\ \text { Helen } & \text { Female } & 89 & 6 \text { months } \\ \text { Joe } & \text { Male } & 88 & 3 \text { months } \\ \text { Bernice } & \text { Female } & 93 & 13 \text { months } \\ \text { Brenda } & \text { Female } & 92 & 21 \text { months } \\ \text { Patrick } & \text { Male } & 77 & 8 \text { months } \\ \text { Belinda } & \text { Female } & 93 & 2 \text { months } \\ \text { Madge } & \text { Female } & 92 & 41 \text { months } \\ \text { Annie } & \text { Female } & 92 & 5 \text { months } \\ \text { Louisa } & \text { Female } & 91 & 14 \text { months }\end{array}$

\subsection{Analytical Framework}

The interviews were transcribed, and a thematic analysis was undertaken using an iterative approach between the data and the literature. Thematic analysis is a method for identifying, 
analysing and reporting patterns within data, and a theme captures something important about the data in relation to the research question $[64,65]$. As such, theoretical thematic analysis is driven by the researcher's theoretical interests [66] - in this case positive ageing and people in the fourth age living in care home environments. We synthesise and apply the subjective indicators imbued in theories of positive ageing which highlight the factors which promote and sustain quality of later life. The premise is that a person is able to maintain a sense of self, belonging and autonomy as they age. Our thematic categories are presented under the following headings: The decision to move to a care home: exercising choice and autonomy during the transition; Living in a homelike environment: facilitating a positive emotional state; Choice and control over daily life: facilitating a positive emotional state; Interaction with others: facilitating opportunities for social investment; Activities/meaningful engagement: facilitating a positive emotional state and opportunities for social investment; Optimising health and well-being through activities: facilitating a positive emotional state; and Quality of life: residents' perspectives.

\section{Findings and Discussion}

\subsection{The Decision to Move to A Care Home: Exercising Choice and Autonomy During the Transition}

As people transition into the fourth age - when moving into long-term care in particular - they often experience drastic life changes. Leaving behind their home and neighbourhood and experiencing personal losses [67] often occurs in conjunction with a loss of independence and a decline in physical and mental health [68]. When residents talked about their lives before moving to Belong, they often focused on the agency and independence they were able to exercise and the activities they engaged with prior to needing care and support. For Bernice, this involved flying down to see her sister's children; for Joe, being active and happily retired; and for Norma being actively involved in the church. However, often an abrupt physical change impacts and means that such activities and autonomy are no longer possible. Bernice accepted and adjusted to this and now 'the telephone must do' to maintain contact with her family now she can no longer travel to see them in person. Norma remains as involved as she can be with the limitations imposed by her physical condition, and Joe 'adjusted', realising what he 'can and can't do':

I used to ... fly down to see [my sister's] children in Devon and Cornwall because we've always kept in touch. But I can't do that as much as I used to now, so the telephone must do. (Bernice, 93)

I was happily retired, I was very active, up until about four years ago I used to dance a lot. And then all of a sudden I just started to deteriorate quite quickly, and so I adjusted things you know slowing down and stuff and I think you start to realise what you can and can't do but I was alright up until about five or six weeks ago until I had a couple of falls and they really set me back. (Joe, 88)

Oh well I was very involved in the church, I used to get involved in everything there... different services and coffee mornings... I got out and about yes. You see after I'd had my fall I couldn't walk so I couldn't do that stuff, I can't go without my frame now... get involved in as much as I can... and of course I can only do certain things because I can't see. (Norma, 97) 
For many people, the actual admission to a care home is usually at the time of crisis, often following hospitalisation or a fall [69]. Such an experience then, has the potential to be traumatic and disruptive and therefore potentially unconducive with positive ageing. In all cases for the participants in this study, some health issue or crisis precipitated the move to Belong. Among those for whom the decision to move was taken by the resident themselves, or jointly with family members, it appeared to be a more positive experience as they had some control and autonomy over the process. However, that is not to say that the move was without difficulty and there was a recognition that help to maintain quality of life was needed and the ability to adapt appears to be key.

Annie made the decision to move into Belong (in conjunction with her family) after feeling that the care arrangements in her original home were no longer satisfactory. Annie adapted to her new circumstances and ultimately took control of the whole process, although her family 'all agreed' with the decision for her to move to a care facility. It was she however, who 'decided', 'rang for a brochure', 'thought it looked nice' and 'made an appointment'. In this way, Annie exercised agency and autonomy about the decision and the move to Belong, leading her to have 'no regrets at all':

'We all agreed it was good, so l've no regrets at all. I feel more settled and more organised now I'm here, you know when I was at home there were so many different people coming in and out to look after me and it just wasn't suitable... I decided that l'd better get myself into a home, and I rang here for a brochure and thought it looked nice, so I made an appointment to look around and I came, and I liked it'. (Annie, 92)

For Helen, following her diagnosis and treatment for cancer, it became apparent that she would have to alter her original plan to move into sheltered accommodation as she needed more care and support than would have been available there. It was 'obvious' to Helen that she needed additional care and she exercised agency in making the decision to move to Belong where she would receive appropriate levels of care:

I was thinking about going to into care but more like sheltered housing type of thing but then I got cancer, so I moved into a care home that was nearer [the] hospital. So, I was booked in for six weeks, four weeks treatment and two weeks recovery but I didn't recover, it took me a long time to get back on my feet. So, it was just obvious that I couldn't just go to sheltered, I needed more'. (Helen, 89)

Similarly, Alice who broke her hip following a fall recognised she could no longer manage to live independently. However, the transition for her was not easy, being unable to return to her flat was 'very disappointing'. Alice appears to be less positive about her change in circumstances and instead was reluctantly resigned to the change in circumstances - moving to a care home:

'I fell in my flat last year and broke my hip so that's why I am here, and I can't go back to my flat which is very disappointing'. (Alice, 80)

Belinda also recognised that she needed additional care and support and could no longer live independently, and like Alice she appeared to be disappointed by this. Belinda's use of - 'I know/I'm very aware/I'm not stupid - when she talks about her change in circumstances shows clear evidence of her agency, autonomy and independence of spirit. Additionally, Belinda's use of 'I can pay for it' reinforces her recognition that although she cannot continue to live independently, she remains independently minded, financially independent and therefore able to exercise a degree of control through being able to 'ask for help': 
'I know I need a lot of help. I couldn't be on my own now, I had a house, but I've sold it now, there's no point you see...I'm very aware that I will need more help soon, I'm not stupid. I can always ask for help, and I will, and I can pay for it'. (Belinda, 92)

Rose felt that move to Belong was best for her son and daughter as they thought it was the safest place for her. However, Rose did not appear to be happy with the decision to move or to feel that she had exercised control over the decision, or that it was necessarily in her best interests:

'I think my son and daughter felt this was the safest place for me, it was best for them'. (Rose, 80)

It appears that the circumstances of people moving into a care home environment are significant. Such transitions often involve moving after a crisis of some kind (illness/a fall/ loss of capacity) and in these circumstances agency and autonomy could be compromised. The most positive outcome appears to be where the transition to a care home can be managed as much as possible to help older people work adapt to their changed circumstances. In this way, care providers can play a role in countering the ramifications associated with moving into a care home. Evidence suggests that where an admission is planned and where residents are involved in the decision-making process, the adjustment to care is much easier [70]. The use of a life/care plan during the transition to a care home environment has the potential to facilitate autonomy and choice and to promote continuity regarding how life was lived before. When a resident moves into Belong, an electronic care plan is created in the Person Centred Software (PCS). This plan is created through the completion of several risk- and care assessment tools, in addition to an initial assessment during which questions are asked about a resident's emotional state and social preferences. Information is gathered about things which capture what a resident's life looked like before moving into Belong, such as important life events, social interests and hobbies, daily routines, skills, likes and dislikes. In this way, staff can easily become aware of things that residents might like to do or talk about. Members of staff can add information to the plan at any time as a situation changes and all aspects of the care plan have review cycles to ensure that the plan is kept up-to-date.

\subsection{Living in a Homelike Environment: Facilitating a Positive Emotional State}

A welcoming, homelike environment and culture in a care home context are integral to promoting positive ageing among older people [30]. Belong embodies the philosophies of the culture change movement that underpin resident-focused practice which emphasises a home for life and continuity of engagement and quality of life. Christine talked extremely positively about Belong's ethos:

'I love the philosophy of the place. I love the idea that you're still living in a community, you're not just one person sat on a chair in this great big room. People treat you like people, this is your home, you know... this philosophy and this kind of care needs to become the norm'. (Christine, Alfred's (74) wife)

Residents and their relatives reported that the smaller scale household design in Belong was more homelike than traditional care home configurations:

"I like the layout of the households with the lounge area, the television area and the dining area'. (Ralph, Elsie's (85) son)

'I like that its smaller than others, I'm not one for crowds'. (Nigel, 86) 
Care home environments that provide a comfortable balance of private and community spaces and are associated with a higher quality of life [59]. In addition to the positive culture and ethos in Belong, residents and their family members all talked very positively about how Belong felt more 'like home' rather than like an institution:

'I came here presuming it would be you know like home really and that's how I feel, like home. It's lovely'. (Sandra, 69)

'I settled in ok... I feel safe here, it's a home it's not an institution. There's no rules... it's like being part of the family'. (Belinda, 92)

Resident's family members talked about their relatives being 'settled' in Belong suggesting feeling or seeming at home. This also suggests that the transition from previous living arrangements to the care home environment was a positive one:

'Dad has settled in lovely here...it's great. (Sally, Nigel's (86) daughter)

'[My mother is] far more settled here'. (Ralph, Elsie's (85) son)

Within traditional care home settings with shared bedrooms and bathrooms, there is limited space for residents' personal belongings which negatively impacts on preservation of residents' identity [59]. Residents and family members highlighted the positive effects of having personal belongings around in terms of it being 'homely' or 'homelike':

'Yes, I do [like my room]. I'm going to get a few more things in to make it homely... it feels like my home now'. (Annie, 92)

'It's as homelike as it can be... their own rooms are their own rooms and if they want to bring their own stuff in they can... They can bring their own pictures, their own TV, music or whatever and the rooms are made as homely as possible'. (Don, Margot's (79) husband)

Feedback from household residents suggests therefore that living in a small-scale, homelike environment as operated by Belong has benefits in terms of facilitating positive impacts on emotional states, represented by reports of feeling 'at home' and 'being settled'. The design and ethos of Belong - a departure from traditional institutional arrangements - also has beneficial outcomes on experiences of positive ageing from the perspective of residents.

\subsection{Choice and Control over Daily Life: Facilitating a Positive Emotional State}

Being able to have control and autonomy also relates to older people's continued engagement in their normal, everyday activities and maintenance of their usual routines, for example, waking and going to bed when they choose and dressing how they like [36]. Residents talked very positively about being able to exercise choice over their daily lives, in terms of going to bed/waking up; mealtimes; and having their personal belongings around them. For Nigel, being able to play board games whenever he wanted was positive:

'You can get up in the middle of the night and have a game [of draughts] you know ...you can do anything you like if you want' (Nigel, 86)

Having choice over bedtime and waking was also raised as being important in maintaining continuity [38], although Nigel acknowledged that his choices were facilitated - for example he is 'put to bed':

You can go to bed at night about 7 or 8 o'clock or 9 o'clock...they put you to bed at a certain time and get you up at a certain time... you don't get up early if you don't want...you might 
be an hour later than everyone else, it doesn't matter...there's no rushing any of us...you have choice about everything' (Nigel, 86)

'She gets up when she wants to get up from what I can gather between half nine and half eleven, and then someone comes to see her after lunch'. (Ralph, Margot's son)

Flexibility and resident-led practices include the timings of meals, where residents are able to eat (in the privacy of their room or in the communal area) and the type of food available. Residents talked positively about being able to exercise choice over mealtimes, in terms of where meals are eaten, what is eaten and when meals are taken. Nigel recognised the benefits of eating in the communal area but acknowledged that he could eat in his own room if he chose, which Bernice preferred:

'You can have your [meals] in here (own room) if you're not well, but if you are well you should be out joining in...you have a good choice of food here as well'. (Nigel, 86).

'I've got shaky hands, and it's embarrassing for me, so I eat in here (her room not the communal household dining area). They don't mind, I asked ...if I could dine alone and ...that's fine'. (Bernice, 93)

Ralph, Margot's son spoke positively about how flexible the staff were regarding mealtimes, highlighting that his mother was never pressurised to eat:

'They don't force food down her, they try to encourage her to eat ...if my mum isn't hungry they don't kick up a fuss and make her eat it there and then, they just give it to her an hour later so there's no pressure'. (Ralph, Margot's son)

Similarly, Christine talked very positively about the efforts made to maintain her husband's dignity and regarding the way his food was presented and served:

'They go out of the way he's got his own menu ... he has pureed food now but it's laid out like a proper meal even though he can't see it really'. (Christine, Alfred's (76) wife)

Preserving identity also relates to older people having privacy, having their own personal space with their belongings around them, and feeling known and valued as an individual. Residents and family members acknowledged the importance of this:

'You can have your own things around you and you can pick your clothes'. (Sandra, 69)

\subsection{Interaction with Others: Facilitating Opportunities for Social Investment}

The physical space and environment of Belong appears to have a positive impact on facilitating opportunities for social interaction through which positive relationships can be developed and sustained [55] and positive ageing in a care home context also encompasses the opportunity to participate in social participation (23). Residents talked about the opportunities for social inclusion, being able to avoid loneliness and having opportunities to make friends and interact with others:

'I was lonely [before I came here] with my husband going and my mum and dad going...I enjoy the company'. (Sandra, 69)

'I eat my meals in the communal part of the household, I've got two friends I sit with. (Belinda, 92)

'I've made many many friends here. I've made many enemies as well' [laughs]... When I go downstairs they say "oh hello Nigel". Everybody knows who I am'. (Nigel, 86) 
In Belong there appear to be opportunities for continued interaction and engagement; there are more people around and again the layout of the household and village is significant in reducing isolation, encouraging companionship and interaction:

'Well I particularly like the bistro downstairs because it means when we come and see my mum, walk her down to the bistro she sees people, well people see her and they say 'Hello Elsie and how are you?' and she enjoys that. (Ralph Elsie's (85) son)

The physical space and environment of Belong also appears to have a positive impact on neighbourhood integration (23), and residents welcomed the opportunity to engage with the public:

'Yes, I think that's a good idea that the bistro and the gym are open to the public...in fact it's been nice because if my friends were around and they'd pop in downstairs they would just send a message to say they were here and I could just pop down if I wanted to'. (Brenda, 92)

Being able to exercise choice and autonomy over daily life - in other words not being regimented or restricted - was also reported as being important by residents in terms of promoting a positive emotional state and maintaining quality of life which encompasses positive ageing.

\subsection{Activities/Meaningful Engagement: Facilitating a Positive Emotional State and Opportunities for Social Investment}

Positive ageing in a care home context is also associated the opportunity to participate in meaningful activity [30] and residents talked about being able to enjoy activities, such as gardening, music, educational activities and outings if they chose to:

'I do a bit of gardening...I go to the music'. (Sandra, 69)

'The activities are very good... I go to the ones I fancy...more educational things, a variety of things really ...I went in a coach one time to a ... big lake in Yorkshire, it was a lovely day. I was with another lady in a wheelchair around the lake it was lovely'. (Bernice, 93)

'Then there are some activities, I go to some of them, and then lunch. (Helen, 89)

Again, Nigel acknowledged that being able to participate in activities is facilitated by Belong staff (they wheel us in) and he is able to continue to enjoy watching Manchester United football matches on satellite TV with his friend in the household.

'[l enjoy] watching united play on the tv. They wheel us into the bedroom you know. If they're playing a big match we can sit in that corner in that chair and then prop another chair round here, because some of the television doesn't include united does it?' (Nigel, 86)

Positive ageing in a care home context is also associated the opportunity to find a sense of usefulness [30], and some residents and their family members felt this was achieved through being involved in chosen activities:

'I've found things to do, one of the things they do is that I operate the sweet trolley. I take the trolley around twice a week, sweets, chocolates, toothpaste, lots of things. I go to the gym every day. The other thing I do is I look after the plants on the balcony here'. (Patrick, 77) 'They also let her have a go with the hoover and the duster, tidy the tables up and wash the dishes...it makes a big difference to her, she thinks she's helping out and being part of it here'. (Ralph, Elsie's (85) son) 
Being able to participate in activities and meaningful engagement appears to fulfil two purposes: the maintenance of a positive emotional state and providing opportunities for social interaction - both associated with positive ageing. Even if participation in activities and engagement was facilitated by Belong - as part of their customer commitments and personcentred ethos, the benefits were reported by residents.

\subsection{Optimising Health and Well-Being through Activities: Facilitating a Positive Emotional State}

Optimising health and well-being are important features of positive ageing and Helen and Annie talked positively about how living in Belong helped them to remain active. For both women this appeared to centre on exercise and physical activity which they acknowledged was beneficial to their health and emotional well-being. For Helen, this involved rehabilitation after a fall and for Annie taking exercise for the first time:

'They encourage you not to give up on, to keep going which is good...Like keeping active but also resting if you want to rest'. (Helen, 89)

'I go on the bike, it's a good challenge for me... yes because I wasn't doing any exercise beforehand so that's good'. (Annie, 92)

\subsection{Quality of Life: Residents' Perspectives}

As discussed above, the overarching goal of culture change is to offer homelike environments to older people in which maximum quality of life can be achieved for residents [44, 45]. Quality of life is linked to positive ageing as we are using it - being able to maintain a positive emotional state and opportunities for social interaction. This is best understood when taking account of older people's interpretations, expectations and experiences of positive ageing in their own lives [28]. Most residents talked positively about the impacts on their quality of life since moving to Belong and indicated that it had improved since moving from their previous living arrangements. Feeling safe, being looked after and having their needs met, being well-fed and having company were all cited as enhancing quality of life by residents, including Joe:

'Well I'd say I've got a very good quality of life, I'm looked after, I'm safe and I'm well fed in good staff company.' (Joe, 88)

For Alice, since moving to Belong she is able to do more than she could while living alone as she felt 'safe', was no longer 'scared of falling' as help is on hand if she needed it. Through feeling safe, Alice felt that her dignity is preserved which positively impacts on her quality of life:

'It's about maintaining my dignity, you see I can't walk without my frame and I'm always scared of falling...Also, what's important is feeling safe because if I do fall I know someone will help me'. (Alice, 80)

Similarly, for Helen, being in Belong enables her 'to cope' - in other words exercise some control and autonomy over her life- which she equates with a good quality of life:

'I think quality of life for me is that I'm able to cope sort of thing, and that I feel safe and I

think I do here really'. (Helen, 89)

Annie was very clear that her quality of life had improved because she had moved to Belong. She recognised that she was 'struggling' in her previous home and now this is facilitated, illustrated by Annie's comment 'all that stuff I can do because I'm here'. 
'I do feel as though I have a quality of life now that I'm here...so yes, all that stuff I can do because I'm here ... than if I was at home struggling'. (Annie, 92)

\section{Conclusions}

In this paper we presented new data generated from qualitative interviews with a sample of household residents in the fourth age living in Belong. From the evidence presented above - drawn from the subjective accounts of residents' experiences - it is evident that the household model as operated at Belong does facilitate the maintenance of a sense of self and personal identity, promoting positive emotional states and facilitating social investment, which are subjective indicators of positive ageing. Residents and their family members talked about being known as an individual and feeling that choice and autonomy are to a very large extent - and within the context of physical and sometimes cognitive impairment - facilitated and maintained, again contributing to positive ageing.

We have shown that the household model as operated at Belong facilitates the maintenance of autonomy and independence among residents and that this contributes to positive ageing and quality of life as they define it. However, it should be acknowledged that for people in the Fourth Age, often, autonomy and independence must be enabled or facilitated alongside physical and bodily decline and needs to be understood within the context of such changing circumstances. There are key junctures where these adaptations can be made, with the decision to move to a care facility being particularly significant. It seems that those people who exercised a degree of control over this process were the happiest with the decision taken, and the most likely to report experiences of positive ageing. For service providers, understanding the life of the person before the need for care is crucial in facilitating quality of life and positive ageing. Additionally, assisting with the transition to a household - maintaining as much independence and autonomy as possible - while also facilitating an understanding of how changed circumstances impact on the ability to maintain continuity are key interventions for service providers who subscribe to the culture change ethos.

Living in small scale households with a resident-led ethos as operated under the Belong model appears to have significant positive impacts on older people's quality of life and wellbeing. Not feeling 'institutionalised' and living in a homelike environment can also make the transition to a care home context easier to navigate. Higher staff ratios where residents feel known by staff also seems to contribute to this, as does the design of the households which facilitates a homelike atmosphere and provides opportunities for interaction with others. Encouraging choice and control over meals, daily activities and opportunities for household, village and neighbourhood interaction all appear to have positive impacts on residents' quality of life and ability to age positively. Thus, despite a physical and mental decline in this age, it seems clear that positive/active ageing can both be useful and applicable to people in the fourth age who are living in a care home context and there is a significant role for the household model of care provision in facilitating this. 


\section{Acknowledgments}

The authors would like to thank the residents and family members who participated in the research underpinning the paper. Thanks also to Belong staff who assisted with access to the research participants.

\section{Author Contributions}

Ahmed is the lead author and was responsible for leading the writing of the paper and data analysis. Ormandy contributed to writing the paper and analysis of the data. Seekles contributed to writing the paper and the literature review.

\section{Funding}

The wider study upon which this paper is based is a Knowledge Exchange, jointly funded by Belong Ltd and the University of Salford, UK.

\section{Competing Interests}

The authors have declared that no competing interests exist.

\section{References}

1. Office of National Statistics. Overview of the UK population - Office for National Statistics. 2017.

2. Office for National Statistics. Life Expectancy at Birth and at Age 65 by Local Areas in England and Wales: 2011- 2013. 2014.

3. Higgs P, Gilleard CJ. Rethinking old age: Theorising the fourth age. Basingstoke: Palgrave. 2015.

4. Laslett P. The Third Age, The Fourth Age and The Future. Ageing Soc. 1994; 14: 436-447.

5. Barnes SF. Third Age-The Golden Years of Adulthood. 2011.

6. Ahmed A. Retiring to Spain women's narratives of nostalgia, belonging and community. Bristol: Policy Press. 2015; 209.

7. Ahmed A, Hall K. Negotiating the Challenges of Aging as a British Migrant in Spain. GeroPsych (Bern). 2016; 29: 105-114.

8. Riediger M, Freund AM, Baltes PB. Managing life through personal goals: Intergoal facilitation and intensity of goal pursuit in younger and older adulthood. J Gerontol B Psychol Sci Soc Sci. 2005; 60: P84-91.

9. Singer $T$, Verhaeghen $P$, Ghisletta $P$, Lindenberger $U$, Baltes $P B$. The fate of cognition in very old age: Six-year longitudinal findings in the Berlin Aging Study (BASE). Psychol Aging. 2003; 18: 318-331.

10. Gilleard C, Higgs P. Aging without agency: Theorizing the fourth age. Aging Ment Health. 2010; 14: 121-128.

11. Baltes PB. On the incomplete architecture of human ontogeny. Am Psychol. 1997; 52: 366-380.

12. Baltes PB, Smith J. New frontiers in the future of aging: From successful aging of the young old to the dilemmas of the fourth age. Gerontology. 2003; 49: 123-135. 
13. Fernandez-Ballesteros R. Positive ageing: Objective, subjective, and combined outcomes. Electron J Appl Psychol. 2011; 7: 22-30.

14. Glasby J, Miller R, Needham C. Adult Social Care. In: Foster L, Brunton A, Deeming C, Haux T, editors. In Defence of Welfare. 2nd ed. Social Policy Association; 2015 p. 94-97.

15. Belong. Belong Villages: The professional dimension. A guide for those with a professional interest in social care. 2017.

16. Belong Itd. Our Vision | Belong Villages. 2018.

17. Jilek R. Residential aged care: A sociological perspective. Geriaction. 2000; 18: 16-20.

18. Belong Itd. Our awards | Belong Villages. 2018.

19. Fernández-Ballesteros R, Molina MA, Schettini R, Santacreu M. The semantic network of aging well. In: Robine J, Jagger C, Crimmings E, editors. Annual review of gerontology and geriatrics. Springer Publishing Company; 2013. p. 79-107.

20. Franklin NC, Tate CA. Lifestyle and Successful Aging: An Overview. Am J Lifestyle Med. 2009; 3: 6-11.

21. Foster L, Walker A. Active and Successful Aging: A European Policy Perspective. Gerontologist. 2015; 55: 83-90.

22. Strawbridge WJ, Wallhagen MI. Self-Reported successful aging: correlates and predictors. In: Poon LW, Gueldner SH, Sprouse B, editors. Succesful aging and adaptation with chronic diseases in older adulthood. New York: Springer; 2003. p. 1-24.

23. Strawbridge WJ, Wallhagen MI, Cohen RD. Successful aging and well-being: Self-rated compared with Rowe and Kahn. Gerontologist. 2002; 42: 727-733.

24. Estes CL, Binney EA. The biomedicalization of aging: dangers and dilemmas. Gerontologist. 1989; 29: 587-596.

25. Koch T, Webb C. The biomedical construction of ageing: Implications for nursing care of older people. J Adv Nurs. 1996; 23: 954-959.

26. World Health Organization (WHO). Active Ageing: a policy framework. Geneva; 2002.

27. Economic \& Social Research Council. Growing older: Research and Policy. 2001.

28. Walker A, Foster L. Active ageing: Rhetoric, theory and practice. In: Ervik R, Skogedal-Linden T, editors. The Making of Ageing Policy: Theory and Practice. Cheltenham: Edward Elgar; 2013. p. 27-53.

29. Walker A. A strategy for active ageing. Int Soc Secur Rev. 2002; 55: 121-139.

30. Van Malderen L, Mets T, De Vriendt P, Gorus E. The active ageing-concept translated to the residential long-term care. Qual Life Res. 2013; 22: 929-937.

31. McDonald L. Theorising about ageing, family and immigration. Ageing and Society. Cambridge University Press; 2011; 31: p. 1180-1201.

32. Rowe J, Kahn R. Succesful Aging. Gerontologist. 1997; 37: 433-440.

33. Johnson KJ, Mutchler JE. The emergence of a positive gerontology: From disengagement to social involvement. Gerontologist. 2014; 54: 93-100.

34. Gerritsen DL, Steverink N, Ooms ME, Ribbe MW. Finding a useful conceptual basis for enhancing the quality of life of nursing home residents. Qual Life Res. 2004; 13: 611-624.

35. Help the Aged. My home life quality of life in care homes. A review of the literature. London; 2007.

36. Cooney A. 'Finding home': A grounded theory on how older people 'find home' in long-term care settings. Int J Older People Nurs. 2012; 7: 188-199. 
37. Nolan M, Brown J, Davies S, Keady J. The senses framework : Improving care for older people through a relationship-centred approach. University of Sheffield; 2006. 1-152 p.

38. Blood I. Older people with high support needs: How can we empower them to enjoy a better life. 2010; 1-16.

39. Grabowski DC, O'Malley AJ, Afendulis CC, Caudry DJ, Elliot A, Zimmerman S. Culture change and nursing home quality of care. Gerontologist. 2014; 54: S35-45.

40. Zimmerman S, Cohen LW. Evidence behind The Green House and similar models of nursing home care. Aging Health. 2010; 6: 717-737.

41. Weiner AS, Ronch JL. Culture change in long-term care. Binghamton: Haworth Press; 2003.

42. Koren MJ. Person-centered care for nursing home residents: The culture-change movement. Health Aff. 2010; 29: 312-317.

43. Fagan RM. Pioneer Network: Changing the culture of aging in America. J Soc Work Long-Term Care. 2003;2: 125-140.

44. Brune K. Culture change in long term care services: Eden-Greenhouse-Aging in the Community. Educ Gerontol. 2011; 37: 506-525.

45. The Pioneer Network. What is Culture Change? 2018.

46. Thomas WH. Life worth living: How someone you love can still enjoy life in a nursing home: the Eden alternative in action. VanderWyk Burnham; 1996. 208 p.

47. Coleman MT, Looney S, O'Brien J, Ziegler C, Pastorino CA, Turner C. The Eden Alternative: Findings after 1 year of implementation. J Gerontol A Biol Sci Med Sci. 2002; 57: M422-427.

48. Ransom S. Eden Alternative: The Texas Project. 2000.

49. Bergman-Evans B. Beyond the basics. Effects of the Eden Alternative model on quality of life issues. J Gerontol Nurs. 2004; 30: 27-34.

50. Rosher RB, Robinson S. Impact of the Eden Alternative on family satisfaction. J Am Med Dir Assoc. 2005; 6: 189-193.

51. White-Chu EF, Graves WJ, Godfrey SM, Bonner A, Sloane P. Beyond the medical model: The culture change revolution in long-term care. J Am Med Dir Assoc. 2009; 10: 370-378.

52. Kane RA, Lum TY, Cutler $\amalg$, Degenholtz HB, Yu TC. Resident outcomes in small-house nursing homes: A longitudinal evaluation of the Initial Green House Program. J Am Geriatr Soc. 2007; 55: 832-839.

53. Thomas WH, Johansson C. Elderhood in Eden. Top Geriatr Rehabil. 2003; 19: p. 282-290.

54. Green DA. Conceptualization and development of the household/neighborhood model for skilled nursing facilities: A case study. Front Archit Res. 2014; 3: 228-237.

55. Nelson G. Household Models for Nursing Home Environments. Nelson. Tremain Partnership; 2016.

56. Shields S, Norton L. In Pursuit of the Sunbeam: A Practical Guide to Transformation from Institution to Household. Milwaukee: Action Pact Press; 2006. 208 p.

57. Cohen-Mansfield J, Bester A. Flexibility as a management principle in dementia care: The Adards example. Gerontologist. 2006; 46: 540-544.

58. Andersen E, Smith M, Havaei F. Nursing home models and modes of service delivery: Review of outcomes. Heal Aging Res. 2014; 3: 1-11.

59. Fleming R, Goodenough B, Low LF, Chenoweth L, Brodaty H. The relationship between the quality of the built environment and the quality of life of people with dementia in residential care. Dementia. 2016; 15: 663-680. 
60. Ausserhofer D, Deschodt M, De Geest S, van Achterberg T, Meyer G, Verbeek H, et al. "There's no place like home": A scoping review on the impact of homelike residential care models on resident-, family-, and staff-related outcomes. J Am Med Dir Assoc. 2016; 17: 685-693.

61. Verbeek H, van Rossum E, Zwakhalen SM, Kempen GI, Hamers JP. Small, homelike care environments for older people with dementia: A literature review. Int Psychogeriatr. 2009; 21: 252-264.

62. Calkins MP. Ten senior living design innovations. I Advance Senior Care. 2011.

63. Morgan-Brown M, Newton R, Ormerod M. Engaging life in two Irish nursing home units for people with dementia: Quantitative comparisons before and after implementing household environments. Aging Ment Health. 2013; 17: 57-65.

64. Ahmed A, Quraishi M, Abdillahi A. Optimising rigor in Focus Group Analysis: Using content/thematic and form/structural approaches to understand British Somali' s experiences of policing in London. Int Soc Sci Rev. 2017; 93: art.3.

65. Braun V, Clarke V. Using thematic analysis in psychology. Qual Res Psychol. 2006; 3: 77-101.

66. Warr DJ. "It was fun... but we don't usually talk about these things": Analyzing Sociable Interaction in Focus Groups. Qual Inq. 2005; 11: 200-225.

67. Riedl M, Mantovan F, Them C. Being a nursing home resident: A challenge to One's Identity. Nurs Res Pract. 2013; 2013: 932381.

68. Cohen-Mansfield J, Wirtz PW. The reasons for nursing home entry in an adult day care population: Caregiver reports versus regression results. J Geriatr Psychiatry Neurol. 2009; 22: 274-281.

69. Davies S, Nolan M. "Making the best of things": Relatives experiences of decisions about carehome entry. Ageing Soc. 2003; 23: 429-450.

70. Davies SM. Relatives' experiences of nursing home entry : a constructivist inquiry. University of Sheffield; 2001.

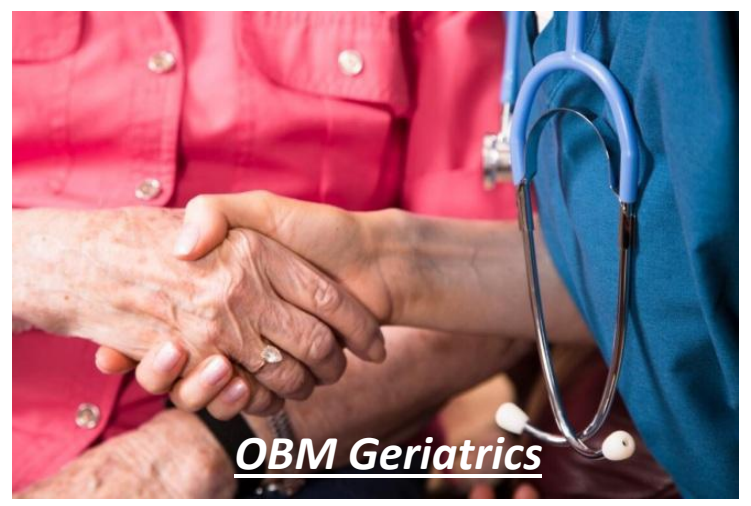

Enjoy OBM Geriatrics by:

1. Submitting a manuscript

2. Joining in volunteer reviewer bank

3. Joining Editorial Board

4. Guest editing a special issue

For more details, please visit: http://www.lidsen.com/journals/geriatrics 
Research Article

\title{
Aging and the Art of Happiness: Time Effects of A Positive Psychology Program with Older Adults
}

Elizabeth Orsega-Smith ${ }^{*}{ }^{\dagger}$, Stephen Goodwin ${ }^{\dagger}$, Melissa Ziegler, Katie Greenawalt, Jennie Turner, Erica Rathie

Department of Behavioral Health \& Nutrition, Carpenter Sports Building, Newark, Delaware, University of Delaware, United States of America; E-Mails: eosmith@udel.edu; goody@udel.edu; Mlz@udel.edu; kgreenaw@udel.edu; ilturner@udel.edu; ejrathie@udel.edu

+ These authors contributed equally to this work.

* Correspondence: Elizabeth Orsega-Smith; E-Mail: eosmith@udel.edu

Academic Editor: Lisa Hollis-Sawyer

Special Issue: Got Aging? Examining Later-life Development from a Positive Aging Perspective

OBM Geriatrics

2019, volume 3 , issue 1

doi:10.21926/obm.geriatr.1901029
Received: October 31, 2018

Accepted: January 07, 2019

Published: January 10, 2019

\begin{abstract}
Background: Research has shown that positive psychology interventions can enhance subjective well-being and reduce depression. However, the efficacy of these programs with older adult populations has been minimally studied. The present study studied the short and long term impact of an intervention enhancing happiness and overall mental well-being in older adults.

Methods: The Art of Happiness is an 8-week program conducted at 2 senior centers in the state of Delaware. Each 90-minute class examined a different topic; (1) defining happiness, (2) stress management, (3) reflecting on happiness, (4) compassion and human connection, (5) forgiveness, (6) transforming suffering, (7) mindfulness, and (8) humor. Pre, post, and 6 months post program questionnaires assessed participant subjective happiness, stress, life satisfaction, depression, mindfulness, arousal states, and general demographic and health information.
\end{abstract}

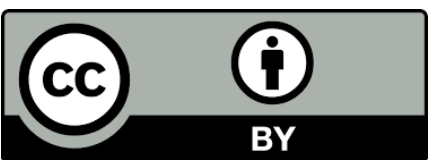

(C) 2019 by the author. This is an open access article distributed under the conditions of the Creative Commons by Attribution License, which permits unrestricted use, distribution, and reproduction in any medium or format, provided the original work is correctly cited. 
Results: 32 participants completed the course and were mostly married (43.8\%), female (87.5\%) and Caucasian (90.6\%), with an age range of 53-84 years (70.91 +.70 years). After controlling for age, gender, and health issues, there was a significant time effect over 6 months post program for happiness, mood state of tiredness, and mindfulness constructs of non-judging and non-reactivity, stress, and satisfaction with life $(p<.05)$. Conclusions: These results suggest that changes in positive mental health have the potential to be maintained in older adults after a positive psychology program ends. It may be that individuals have learned techniques and incorporated them into their lifestyle. Programs emphasizing these aspects of well-being may have potential to buffer the older adult population against poor mental health by improving subjective happiness and mental wellbeing.

\section{Keywords}

Aging; happiness; well-being; positive psychology

\section{Introduction}

The Dalai Lama has stated that the purpose of every human being is to seek happiness. Further, the Dalai Lama believes it is possible to train one's mind to develop those traits that lead to greater happiness. In order to achieve happiness the Buddhist philosophy teaches us that compassion for others, forgiveness, and perspective are central to any happy and fulfilling life. This attitude is also reflected in our western philosophy as almost every parent will express one of their main goals is for their child to be happy [1].

Lyubomirsky [2] has stated $50 \%$ of our happiness is due to genetics, $10 \%$ to our life circumstances, but $40 \%$ is within our control. What we do with that $40 \%$ can have a significant impact on our overall happiness. With that in mind we wanted to examine if we could see any long-term effects on the happiness of older adults after participating in an 8-week course. The course was based upon the teachings of the Dalai Lama as well as positive psychology developed by Dr. Martin Seligman and Dr. Mihaly Csikszentmihalyi. A previous study had shown these lessons had immediate positive effects on the happiness of older adults [3]. The purpose of this study was to see if those initial gains would still be apparent 6 months post intervention.

With approximately 46.2 million Americans currently over the age of 65 , older adults currently represent $14.5 \%$ of the United States population [4]. Furthermore, by 2030 , older adults will comprise $20 \%$ of the population and by 2050 , the number of Americans aged 65 and older will more than double its current number $[4,5]$. As this population suffers from multiple declines in functional abilities and independence, aging is often characterized as a time of loss [6]. Cognitive ability, mental well-being, and physical functioning, all may decline throughout the aging process $[7,8]$. Additionally, chronic diseases which often plague older adults leading to psychological health outcomes $[9,10]$ can result in decreased overall quality of life and sense of well-being and in the older adult population [11].

As the United States population ages, there is an increased demand for mental and behavioral health services. Furthermore, 15 to 20 percent of U.S. adults older than age 65 have experienced 
depression [5, 12]. Depression is not only correlated with increased risk of morbidity, but also a heightened risk of suicide, and declines in physical, cognitive, and social functioning, all of which can lead to increased mortality [13].

Evidence-based health promotion programs targeting older adults are well established, although programming focused on mental health promotion is lacking [14]. The focus of previous evidenced based mental health interventions for older adults have been on those who have been diagnosed with severe depression or dysthymia, are chronically ill, or are home bound under the care of social services. Four evidence-based mental health programs; Healthy IDEAS (Identifying Depression, Empowering Activities for Seniors), PEARLS (Programs to Encourage Active, Rewarding Lives), and IMPACT (Improving Mood-Promoting Access to Collaborative Treatment) program; have been recommended on the National Council on Aging website. Overall, the focus of these programs is to connect frail older adults who are home-bound or clinically depressed, or their care providers to services to assist in improving their quality of life. These programs highlight managing depression or other negative mental health disorders through education of ways to avoid substance abuse or promotion of services and treatment.

Although age related declines may occur, positive traits and characteristics attained through life experience may be protective against mental illness as well as increase positive mental wellbeing in older adults [15-18]. However, we can look to incorporate positive psychological characteristics into programs and treatments to impact aging process. There is some evidence that positive psychology interventions can promote well-being in older adults. In a recent review of positive psychology interventions and older adults, Sutipan and colleagues [18] found that reminiscing seemed to have the greatest effect on quality of life. However, there is no conclusion about a set intervention length or specific types of activities that make the most impact in this population.

Positive psychology is defined as the scientific study of positive experiences and positive individual traits. This concept stems from the belief that human strengths and virtues may act as buffers against mental illness [16, 19]. This discipline emphasizes prevention of illness by fostering positive emotions extending beyond the focus of clinical psychology that may focus on treatment of mental illness to alleviate suffering [16]. Previous research has concluded that positive psychology interventions can develop overall well-being through enhancements in subjective wellbeing, psychological well-being, and happiness and reduce depressive symptoms and negative affect $[21,22]$. While research in the field of positive psychology is growing, few studies have specifically targeted the older adult population; those that have, showed promising results. In a longitudinal study, optimism has predicted lower risk of stroke [23] and participation in positive psychology programs has shown reduction in depressive symptoms and improvements in life satisfaction [17, 24]. In order to foster positive aging and mental well-being in older adult populations further research is suggested.

One particular aspect of positive mental well-being involves the concept of happiness. Happiness can be described as "the experience of joy, contentment, or positive well-being, combined with a sense that one's life is good, meaningful, and worthwhile" [2]. Research in the field of positive psychology and happiness often define a happy person as someone who experiences frequent positive emotions like joy, interest, and pride, and infrequent (though not absent) negative emotions such as sadness, anxiety, and anger. Often, happiness is used 
interchangeably with subjective well-being, and has also been said to be closely related to life satisfaction, appreciation of life, and moments of pleasure [25].

Multiple studies have found that happiness and health go hand-in-hand. Research has found that happiness can improve heart health [26, 27], enhance the immune system [28], reduce disease and disability [29], increase longevity [30,31], and can alleviate pain in the context of disease [32]. Happiness and positive emotions can have a protective effect on health.

Mindfulness is another aspect of positive mental health and can be defined as being in the moment, having non-judgmental awareness, and being non-reactive and non-judgmental [33]. As a practice, mindfulness has been around for many years, but researchers have only recently begun to study its effect on health and well-being. Studies have shown that in older adults mindfulness training can decrease anxiety, improve depressive symptoms [34], improve sleep, reduce pain, and improve well-being [35]. Not only can mindfulness help an individual reach an inner peace but can also bring about changes in mental health, physical pain, and overall happiness resulting in positive effect on older adults.

Another construct that has been shown to have a significant impact on one's happiness is forgiveness. While we did not measure forgiveness it was an important topic discussed in the course. According to the Greater Good Science Center at UC Berkely [36], those who are able to forgive others have more happiness and more positive health outcomes. Forgiveness is significant in that when one is able to forgive another person it allows them to move on with their life and in some cases rebuild a relationship. Since having strong relationships with others is one of the strongest indicators of happiness [37] the ability to forgive and move on is crucial to many people's happiness.

The concept of mood is multifaceted and challenging to define. In the domain of cognitive psychology, mood results from the appraisal of an emotion, and is thought of as a collection of enduring feelings related to evaluative and cognitive states, which effect all subsequent evaluations, feelings, and actions [38]. Mood has been defined and measured in the literature in terms of positive and negative affect. Positive and negative affect can each be described in terms of bipolar "poles", for example, adjectives describing positive affect such as "active," "energetic," and "lively" represent the high pole, while items like "tired," "sleepy," and "sluggish" represent the low pole [39]. Positive affect can be defined as pleasurable engagement with the environment resulting in happiness, joy, excitement, enthusiasm, and contentment [40]. Individuals who score high in positive affect are more likely to describe themselves as enthusiastic, confident, and motivated than people who score low in positive affect [41]. Happy people have skills and resources that they have acquired over time while experiencing positive moods, making success again more likely [25]. Hence, positive affect or positive mood is associated with accomplishments and overall happiness.

According to Martin Seligman, well-being is the basis for positive psychology, a construct required for personal flourishment. Well-being as a construct is operationalized by five measurable concepts: (1) positive emotion (2) engagement (3) meaning (4) positive relationships and (5) accomplishments (PERMA) [42]. Positive emotions are an individual's subjective views on his or her life, and are often measured by life satisfaction. Engagement is the subjective experience of flow state. In flow, an individual becomes absorbed in their task entirely, time seemingly stops or slows and cognitive thought is absent. Meaning is conceptualized by the notion that one is serving something greater than oneself (a higher purpose). Accomplishment is defined 
by the pursuit of success, achievement and/or mastery for one's own personal gain. Positive relationships are the pursuit of meaningful, healthy relationships in one's life. Together, these five elements provide support for personal well-being [42]. PERMA has previously been utilized in positive psychology research, however a lack of experimental application among older adults in the United States warrants further investigation [24]. The Art of Happiness program development was guided by the five measurable concepts of PERMA and the teachings of the Dalai Lama. As the principles of Positive Psychology and those espoused by the Dalai Lama are similar in many respects the course easily blends the science of happiness with the Buddhist philosophy. Martin Seligman's theory of well-being and the 5 concepts has guided our "Art of Happiness" curriculum development.

We were interested in studying the impact of participation in a course based on positive psychology. Our research question was: What is the effect of time (pre-post- 6 months after intervention) controlling for age, gender and health conditions on outcomes of happiness, mindfulness, mood, depression, stress, and satisfaction with life in a population participating in an 8 week positive psychology program.

\section{Materials and Methods}

\subsection{Participants}

Participants were recruited from two local senior centers via word of mouth and distribution of flyers at the centers. Convenience sampling was used to gain participants. A week prior to program implementation, researchers attended three popular senior center events in order to promote the Art of Happiness program. Program participant inclusion criteria included the following: (1) participants were required to be 50 years of age and older, (2) be community dwelling residents, (3) be independently living (without the reliance on another individual to assist with the day to day activities), (4) have the ability to ambulate, (5) could commit to attending the 8 weekly sessions (6) have no severe cognitive impairment (as per self report), (7) no current clinically diagnosed depression, and (8) not reliant on a caregiver. There was no restriction on class size. Participants who completed the pre and post questionnaires and met the minimum requirements of attending six out of the eight classes received a $\$ 25.00$ Visa gift card.

A total of 34 participants agreed to participate and completed the pre and post intervention assessment with a mean age of $70.9 \pm 7.7$ years. The majority of the participants were female (85.3\%), Caucasian (91.2\%), who were mostly married (47.1\%) with some education beyond a high school degree (53.0\%). Over 70\% self-reported that they had high cholesterol or hypertension (Table 1). Due to attrition, not all participants completed assessments at the 6 month follow-up.

The study was approved by the University of Delaware's Institutional Review Board on January, 2016. Prior to beginning the program, all participants were given an informed consent agreement which was then signed. 
Table 1 Descriptive statistics of intervention participants at baseline.

\begin{tabular}{|l|l|}
\hline & Intervention ( $n=34)$ \\
\hline Age, years (mean \pm SD) & $70.91 \pm 7.70$ \\
\hline Sex, \%(n) & $85.3(29)$ \\
Female & $14.7(5)$ \\
Male & \\
\hline Race/Ethnicity, \%(n) & $91.2(31)$ \\
Caucasian & $8.8(3)$ \\
African American & \\
\hline Marital Status, \%(n) & $17.6(6)$ \\
Single & $47.1(16)$ \\
Married & $14.7(5)$ \\
Separated/divorced & $20.6(7)$ \\
Widowed & \\
\hline Highest Level of Education, \%(n) & $44.1(15)$ \\
High school diploma or less & $17.6(6)$ \\
Some college/did not graduate & $11.8(4)$ \\
Technical/vocational school & $17.7(6)$ \\
Associates/bachelor's degree & $5.9(2)$ \\
Graduate school & $2.9(1)$ \\
Other & $51.6(16) n=31$ \\
\hline Health Problems, \%(n) & $18.5(5) n=27$ \\
Overweight/obese & $70.0(21) n=30$ \\
Diabetes & $77.4(24) n=31$ \\
Hypertension & $55.2(16) n=29$ \\
High Cholesterol & \\
Other & \\
\hline
\end{tabular}

\subsection{Measures}

\subsubsection{Demographics}

Participants' demographic information related to gender, age, education level, marital status, ethnicity, and health status was collected. In terms of health information, participants reported if they had ever been told by a doctor they were overweight or obese, had diabetes, had hypertension, had high cholesterol, or had any other illness.

\subsubsection{Subjective Happiness}

The 4 item Subjective Happiness Scale [43] was used to assess subjective happiness. Participants choose one of 7 options such as "In general, I consider myself" (1=not a very happy person, $7=$ a very happy person) or "Some people are generally not very happy. Although they are not depressed, they seem as happy as they might be. To what extent does this characterization 
describe you" (1=not at all, 7=a great deal). The 4 items are summed to create an indicator of level of overall happiness.

\subsubsection{Mindfulness}

Mindfulness was measured using the Five Facet Mindfulness Questionnaire [44]. Thirty-nine items measured 5 components of mindfulness; non-judge, describe, non-react, observe, and act with awareness. Participants respond to the statements using a 5 point Likert scale where $1=$ never or very rarely true and 5= very often or always true. Example items for the subscales are as follows: observe "I notice smells and aromas of things." Describe: "My natural tendency is to put my experiences into words." Act with awareness: "I find myself doing things without paying attention." Non-judge: "I criticize myself for having irrational or inappropriate emotions." Non-react: "When I have distressing thoughts or images, I feel calm soon after." Negatively worded items are reverse scored. A sum of the items is calculated for each subscale. High levels of each specific mindfulness subscale are considered by high scores on each subscale.

\subsubsection{Satisfaction with Life}

The Diener Satisfaction with Life Scale [45] assessed satisfaction with life (SWL). In this 5 item scale participants respond on a 7 point Likert Scale where 1= strongly disagree and $7=$ strongly agree. Example statements include "If I could live my life over, I would change almost nothing", "The conditions of my life are excellent", or "'In most ways my life is ideal". The 5 items are summed and the score indicates one's satisfaction with their life overall. Alpha coefficients have ranged from .79-.87 [46].

\subsubsection{Mood}

To measure mood states, the Activation Deactivation Checklist $[47,48]$ was used. Participants respond to states using a 4 point scale from "definitely feel" to "definitely do not feel". The 4 subscales are: Tired (sleepy, wide-awake, drowsy, wakeful, tired,); Calmness (placid, calm, at-rest, still, quiet); Energetic (active, vigorous, energetic, lively, full-of-pep); and Tension (fearful, jittery, clutched-up, intense, tense); which have been validated via factor analysis [48]. After reverse scoring items, total sum of items for each subscale are calculated.

\subsubsection{Perceived Stress}

Stress was assessed by the 10 item Perceived Stress Scale (PSS) [49]. Participants are presented with potential life situations that they may have encountered over the past month and asked about their thoughts and feelings pertaining to them. Participants respond to how these events are perceived as stressful with a 5 point Likert scale where $0=$ "Never" and 4= "Very often". Example situations include "felt nervous or stressed", "felt that you were on top of things", and "been upset because of something that happened unexpectedly". After reverse scoring some of the positively worded items, and then summing all 10 items, high scores are indicators of high stress. Chronbach alpha has been reported as $>.70$ [50]. 


\subsubsection{Depression}

Depression was assessed by the Patient Health Questionnaire (PhQ-9) [51]. Participants were asked to assess how frequently in the past two weeks they have been experiencing symptoms in 9 different scenarios. Responses were on a 4 point scale from $0=$ not at all and $3=$ nearly every day. Example scenarios are "Feeling tired or having little energy", "Feeling bad about yourself - or that you're a failure or have let yourself or your family down", "Little interest or pleasure in doing things", and "Feeling down, depressed or hopeless". Based on a sum score, minor depression is referred as a score of 10-14, moderate 15-19 and major depression, severe >20 [52].

\subsection{Procedures}

The Art of Happiness is an 8-week program guided by Seligman's PERMA principles and using concepts taught by the Dalai Lama with the goals of enhancing happiness levels and overall mental well-being in older adults. This program implemented at 2 senior centers. The program consisted of once per week 90 minute classes that examined a different weekly topic including; (1) defining happiness, (2) stress management, (3) reflecting on happiness, (4) compassion and human connection, (5) forgiveness, (6) transforming suffering, (7) mindfulness, and (8) humor [2]. Each class session incorporated didactic material via lecture, discussion on homework assignments and readings, and class activities. Participants used the homework activity as a means to reflect on the topic of the week. For example, following the introduction on happiness, participants were asked to complete three unselfish acts of kindness each day for three different people. Similarly, following the lecture on compassion, participants were asked to write down three things for which they were grateful. "The Art of Happiness: A Handbook for Living," 10th anniversary edition by the Dalai Lama and Howard C. Cutler was given to each participant along with a folder for hand-outs and a journal to utilize for homework reflections and class notes. Because weekly homework reflections were not collected, each participant determined the level of disclosure of their thoughts during class discussions. The researchers did not collect these participant journals. Each participant received a handout containing weekly lecture points to take home at the conclusion of every class session. See Table 2 for a week-by-week summary of the intervention. This was previously pilot tested [2] and we then expanded this project.

Table 2 Weekly Intervention Description.

\begin{tabular}{|l|l|l|l|}
\hline & Theme & Purpose & Week \\
\hline 1 & $\begin{array}{l}\text { Introduction } \\
\text { to happiness }\end{array}$ & $\begin{array}{l}\text { Introductions, class structure, } \\
\text { and define happiness. }\end{array}$ & $\begin{array}{l}\text { Activity: Icebreaker-Human Bingo } \\
\text { Homework: Complete three unselfish acts of } \\
\text { kindness each day for three different people } \\
\text { before next class. }\end{array}$ \\
\hline 2 & $\begin{array}{l}\text { Reflecting on } \\
\text { happiness }\end{array}$ & $\begin{array}{l}\text { Identify personal sources of } \\
\text { happiness. The difference } \\
\text { between happiness and } \\
\text { pleasure }\end{array}$ & $\begin{array}{l}\text { Activity: Photo voice- Each participant } \\
\text { provides photos that make them happy } \\
\text { Homework: Each morning, write down 3 } \\
\text { things that you have to look forward to that } \\
\text { day. Write down 3 things at the end of the day }\end{array}$ \\
\hline
\end{tabular}




\begin{tabular}{|c|c|c|c|}
\hline & & & that were good. \\
\hline 3 & $\begin{array}{l}\text { Compassion \& } \\
\text { human } \\
\text { connection }\end{array}$ & $\begin{array}{l}\text { Impact of compassion and } \\
\text { gratitude on happiness. The } \\
\text { importance of human } \\
\text { connection, intimacy, } \\
\text { vulnerability, and sharing }\end{array}$ & $\begin{array}{l}\text { Activity: Each day, write } 3 \text { things for which } \\
\text { you are grateful. } \\
\text { Homework: Hand-write a letter expressing } \\
\text { why someone is important to you }\end{array}$ \\
\hline 4 & $\begin{array}{l}\text { Stress } \\
\text { management }\end{array}$ & $\begin{array}{l}\text { Definition of stress, individual } \\
\text { causes of stress, , and stress } \\
\text { management techniques. }\end{array}$ & $\begin{array}{l}\text { Activity: Therapy Puppies- therapy puppies } \\
\text { are brought in } \\
\text { Homework: Identify life stressors and match } \\
\text { which of Segal's } 5 \text { causes is creating the stress. } \\
\text { How can you restructure your thinking to } \\
\text { reduce your stress? }\end{array}$ \\
\hline 5 & Forgiveness & $\begin{array}{l}\text { Why being able to forgive is } \\
\text { crucial to one's happiness } \\
\text {. }\end{array}$ & $\begin{array}{l}\text { Activity: Group discussion on forgiveness } \\
\text { Homework: Write } 3 \text { letters: } \\
\text { 1. Write a letter apologizing to someone who } \\
\text { you treated unfairly (send) } \\
\text { 2. Write a letter of apology to yourself for } \\
\text { something you regret doing in the past } \\
\text { 3. Write a letter of forgiveness to someone } \\
\text { who has hurt you. (do not send unless you } \\
\text { want) }\end{array}$ \\
\hline 6 & $\begin{array}{l}\text { Transforming } \\
\text { suffering }\end{array}$ & $\begin{array}{l}\text { Dealing with guilt and learning } \\
\text { how our negative experiences } \\
\text { help us grow. }\end{array}$ & $\begin{array}{l}\text { Activity: Savoring- Participants were given a } \\
\text { Saltine cracker and asked to let the cracker } \\
\text { dissolve while noticing flavors that go } \\
\text { unnoticed when eaten quickly } \\
\text { Homework: Reflect on a negative experience } \\
\text { and identify how you have grown from it. }\end{array}$ \\
\hline 7 & $\begin{array}{l}\text { Living in the } \\
\text { present/mindf } \\
\text { ulness }\end{array}$ & $\begin{array}{l}\text { Mindfulness and how living in } \\
\text { the present can increase } \\
\text { happiness. }\end{array}$ & $\begin{array}{l}\text { Activity: Body scan- participants were led } \\
\text { through a meditation that brought their } \\
\text { attention to every part or their body. } \\
\text { Zen Garden- Create a Zen garden. } \\
\text { Homework: Practice mindfulness throughout } \\
\text { the week \& reflect in journal. }\end{array}$ \\
\hline 8 & $\begin{array}{l}\text { Humor \& } \\
\text { Closing }\end{array}$ & $\begin{array}{l}\text { How humor improves one's } \\
\text { wellbeing. Summary and share } \\
\text { those experiences that were } \\
\text { most helpful. }\end{array}$ & $\begin{array}{l}\text { Activity: Participants share funny stories/jokes } \\
\text { with the class }\end{array}$ \\
\hline
\end{tabular}

Participants completed a set of valid and reliable survey instruments before the first class session and then again after the conclusion of the program (after $8^{\text {th }}$ week). These instruments were administered to the participants after they signed the informed consent as required by our human subjects committee. The entire questionnaire took approximately 30 minutes to complete 
and subjects were asked to wait until everyone was done prior to submitting them to the researchers. Additionally, 6 months after completion of the program, participants were asked to return for a booster session. The purpose of holding the booster session was to discuss which lessons have continued to impact their daily lives and to assess the program's continued impact on participant subjective happiness, depressive symptoms, life satisfaction, stress levels, mindfulness, and overall mood. This questionnaire also contained additional open-ended questions including; 1 ) How do you define your happiness? Has your definition changed since taking the course? If so, how is it different? 2) What skills and practices that you learned from the class do you find yourself still using? 3) What are the biggest barriers you face to increasing your own happiness? 4) Do you notice any differences in your overall happiness levels after taking the class? 5) Looking back, is there a topic you wish would have been covered in the course that was not discussed? 6) Do you have any additional comments? Once all participants completed the survey instruments, the 90 minute, "booster" session was conducted.

\subsection{Statistical Analysis}

A repeated measures linear mixed effects model was used to examine changes in each outcome variable across the three study time points (pre-intervention, post-intervention and at the 6-month follow-up) accounting for the correlation between measures on the same subject by directly modeling the covariance structure of the residuals as unstructured. Model assumptions were assessed via graphical examination of the residuals. Tests for mean differences were conducted using the Kenward-Roger approximation for the degrees of freedom and $p$-values comparing the mean response between time points were adjusted for multiple comparisons using the Tukey-Kramer method. All statistical analyses were performed using SAS software, Version 9.4 (SAS Institute, Cary, NC).

\section{Results}

\subsection{Repeated Measures Linear Modeling}

After controlling for age, gender, and health issues, there was a significant time effect for happiness $(F=7.33 ; p<.01)$, mood state of tiredness $(F=18.95 ; p<0.0001)$, and mindfulness constructs of non-judging $(F=5.15 ; p<0.05)$ and non-reactivity $(F=3.74 ; p<0.05)$, stress $(F=8.73$; $p<.01)$, and SWL ( $F=3.58 ; p<.05)$. There were non-significant effects for depression $(F=3.14 ; p=.06)$, Mood states of tension ( $F=3.16 ; p=.06)$, calmness $(F=1.55 ; p=.23)$, and energetic $(F=.92 ; p=.41)$ (see Table 3). Post hoc pairwise comparisons were conducted between time points (post-pre, post -6 months, and pre- 6 months) to examine the specific changes in the measures of interest (Table 4). These results showed that there were significant differences between pre-intervention and 6 months post program in happiness, mood construct of tiredness, and mindfulness aspects of non-judging and non- reactivity. The model adjusted means are displayed in Table 5. Examination of the means show that happiness increased from baseline $(4.82 \pm .33)$ to end of program (5.47 \pm .33 ) and then declined slightly at 6 months post program $(5.24 \pm .31)$. At 6 months, the level of happiness was still significantly greater than the baseline level. Similarly, mood aspect of tiredness was at its greatest at baseline $(13.01 \pm .62)$, declined at end of program $(9.43 \pm .83)$ and then slightly increased at 6 months after program completion $(10.49 \pm .90)$. However, that level at 6 
months post- program remained significantly better than the baseline level of tiredness. The mindful aspect of non-judging steadily increased from baseline $(25.24 \pm 1.55)$ to post- program $(27.48 \pm 1.43)$ to 6 months $(28.15 \pm 1.56)$ as did the mindful aspect of non-reactivity from $20.39 \pm$ 1.03 at baseline to $21.98 \pm .93$ at post program to $23.64 \pm 1.13$ at 6 months. These are the model adjusted means and standard errors.

Table 3 Repeated Measures Mixed Effects Model.

\begin{tabular}{|c|c|c|c|c|c|}
\hline Variable & Effect & NumDF & DenDF & FValue & ProbF \\
\hline \multirow{4}{*}{$\begin{array}{l}\text { Subjective } \\
\text { Happiness }\end{array}$} & Time & 2 & 26.01 & 7.33 & 0.0030 \\
\hline & Gender & 1 & 28.65 & 0.13 & 0.7212 \\
\hline & Age & 1 & 30.63 & 0.05 & 0.8321 \\
\hline & Health concerns & 1 & 29.57 & 1.92 & 0.1766 \\
\hline \multirow{4}{*}{$\begin{array}{l}\text { ADACL: } \\
\text { Tiredness }\end{array}$} & Time & 2 & 22.94 & 18.95 & 0.0000 \\
\hline & Gender & 1 & 28.41 & 0.29 & 0.5918 \\
\hline & Age & 1 & 28.80 & 0.23 & 0.6353 \\
\hline & Health concerns & 1 & 28.62 & 0.94 & 0.3392 \\
\hline \multirow{4}{*}{$\begin{array}{l}\text { Mindfulness: } \\
\text { Nonjudging }\end{array}$} & Time & 2 & 22.15 & 5.15 & 0.0146 \\
\hline & Gender & 1 & 32.35 & 1.51 & 0.2279 \\
\hline & Age & 1 & 30.36 & 0.03 & 0.8691 \\
\hline & Health concerns & 1 & 30.30 & 0.44 & 0.5114 \\
\hline \multirow{4}{*}{$\begin{array}{l}\text { Mindfulness: } \\
\text { Nonreactivity }\end{array}$} & Time & 2 & 18.97 & 3.74 & 0.0428 \\
\hline & Gender & 1 & 18.62 & 0.32 & 0.5804 \\
\hline & Age & 1 & 23.37 & 0.26 & 0.6179 \\
\hline & Health concerns & 1 & 20.47 & 1.23 & 0.2803 \\
\hline
\end{tabular}


Table 4 Comparisons between time points.

\begin{tabular}{|c|c|c|c|c|c|c|c|}
\hline Variable & $\begin{array}{l}\text { Time Point } \\
\text { Comparison }\end{array}$ & $\begin{array}{l}\text { Mean } \\
\text { Difference }\end{array}$ & $\begin{array}{l}\text { Standard } \\
\text { Error }\end{array}$ & $\begin{array}{l}\mathrm{K}-\mathrm{R} \\
\mathrm{DF}\end{array}$ & T Value & $\begin{array}{l}\text { Unadjusted } \\
\text { p-value }\end{array}$ & $\begin{array}{l}\text { Tukey- } \\
\text { Kramer } \\
\text { p-value }\end{array}$ \\
\hline \multirow{3}{*}{$\begin{array}{l}\text { Subjective } \\
\text { Happiness }\end{array}$} & 6 months - post & -0.23 & 0.15 & 21.8 & -1.46 & 0.1587 & 0.3294 \\
\hline & 6 months - pre & 0.42 & 0.20 & 28.7 & 2.09 & 0.0455 & 0.1095 \\
\hline & post-pre & 0.65 & 0.17 & 32.1 & 3.85 & 0.0005 & 0.0015 \\
\hline \multirow{3}{*}{ ADACL: Tiredness } & 6 months - post & 1.06 & 0.62 & 21.2 & 1.72 & 0.1001 & 0.2213 \\
\hline & 6 months - pre & -2.52 & 0.72 & 21.1 & -3.51 & 0.0021 & 0.0056 \\
\hline & post-pre & -3.58 & 0.57 & 30.8 & -6.29 & 0.0000 & 0.0000 \\
\hline \multirow{3}{*}{$\begin{array}{l}\text { Mindfulness: } \\
\text { Nonjudging }\end{array}$} & 6 months - post & 0.66 & 0.92 & 21.6 & 0.72 & 0.4811 & 0.7563 \\
\hline & 6 months - pre & 2.91 & 0.90 & 20.3 & 3.21 & 0.0043 & 0.0114 \\
\hline & post-pre & 2.24 & 0.99 & 31.5 & 2.27 & 0.0301 & 0.0749 \\
\hline \multirow{3}{*}{$\begin{array}{l}\text { Mindfulness: } \\
\text { Nonreactivity }\end{array}$} & 6 months - post & 1.66 & 0.95 & 19.9 & 1.74 & 0.0968 & 0.2144 \\
\hline & 6 months - pre & 3.24 & 1.18 & 13.9 & 2.75 & 0.0159 & 0.0395 \\
\hline & post-pre & 1.58 & 0.75 & 27.8 & 2.11 & 0.0441 & 0.1063 \\
\hline
\end{tabular}


Table 5 Model means by time point.

\begin{tabular}{cccccc}
\hline & & & \multicolumn{3}{c}{ Lower } \\
Variable & Time & & Standard & $95 \%$ & Upper \\
& Point & Mean & Error & Cl & $95 \% \mathrm{Cl}$ \\
\hline \multirow{2}{*}{$\begin{array}{c}\text { Subjective } \\
\text { Happiness }\end{array}$} & 6 months & 5.24 & 0.31 & 4.60 & 5.88 \\
\cline { 2 - 6 } & post & 5.47 & 0.33 & 4.81 & 6.13 \\
\cline { 2 - 6 } & pre & 4.82 & 0.33 & 4.15 & 5.49 \\
\hline \multirow{2}{*}{$\begin{array}{c}\text { ADACL: } \\
\text { Tiredness }\end{array}$} & post & 9.43 & 0.83 & 7.76 & 11.11 \\
\cline { 2 - 6 } & pre & 13.01 & 0.62 & 11.74 & 14.28 \\
\hline \multirow{2}{*}{$\begin{array}{c}\text { Mindfulness: } \\
\text { Non-judging }\end{array}$} & post & 27.48 & 1.43 & 24.58 & 30.39 \\
\cline { 2 - 6 } & pre & 25.24 & 1.55 & 22.11 & 28.37 \\
\hline \multirow{2}{*}{$\begin{array}{c}\text { Mindfulness: } \\
\text { Non- }\end{array}$} & 6 months & 23.64 & 1.13 & 21.31 & 25.97 \\
\cline { 2 - 6 } \begin{tabular}{c} 
poactivity \\
\cline { 2 - 6 }
\end{tabular} & post & 21.98 & 0.93 & 20.07 & 23.88 \\
\cline { 2 - 6 } & pre & 20.39 & 1.03 & 18.30 & 22.49
\end{tabular}

\section{Discussion}

The aim of this study was to examine if the immediate positive impact an intervention had on increasing the happiness of older adults would be sustained over a 6 month period. Self-reported levels of happiness and mindfulness did remain significantly higher than baseline. The topics of defining happiness and reflecting on happiness, stress management, compassion and human connection, forgiveness, mindfulness, and humor were apparently meaningful to the participants and presented in a manner so that they continued applying the lessons to their life after the course was completed. These lessons impacted the older adults in ways that promoted a sense of positive well-being. This is an important concept as happiness has been said to be closely related to life satisfaction, appreciation of life, and moments of pleasure [25]. When participants of the study were asked to define happiness, they mentioned words such as being content, joy, wellbeing, peace, and faith. One participant stated "It is a quiet feeling of joy that I feel behind the eyes and the feeling of well-being ...." Another mentioned, "A state of mind, attitude, being positive, avoiding negativity". These simple concepts of seeking positive experiences while avoiding negative ones has been identified by the Dalai Lama as being fundamental to one's happiness.

The notion that one's level of happiness can be improved and then maintained is an important finding, specifically in the older adult population as they are at higher risk of disease associated with negative psychological states. Happiness related studies have been shown to have a positive 
impact on heart health [26,27] and reduce disease and disability [29]. Furthermore, longitudinal studies have indicated that those with higher levels of happiness tend to live longer [30,31].

One finding worth noting is the impact this program had on depression. Although there was not a significant effect of time $(p=.06)$ there was a significant difference immediately post intervention $(p<0.01$; pre $=15.39 \pm 1.19$, post $=13.09 \pm 0.89)$. This indicates it may be beneficial to provide more booster sessions or skills the participants can develop and employ in their daily lives. It may also be beneficial to send emails reminding them to redo one of the assignments or provide a new assignment for them to apply to their lives. However, it was encouraging to see the levels of selfreported depression were highest at the pre-test $(15.39 \pm 1.19)$, fell at post-test $(13.09 \pm .82)$ and then rose slightly $(13.95 \pm 1.12)$. However, the depression scores are still below the pre-test level. These levels all fall within the category of mild depression [49]. The aim was not to reduce depression, but seeing this decline in self-reported depression scores during the course of the study with an associated increase in happiness scores is worthy of further study.

The significant time effect for the two identified aspects of mindfulness (non-react and nonjudge) is not surprising as the course emphasized learning how to respond to circumstances in a non-judgmental and positive manner. Additionally, we believe it is possible that participants became more mindful since mindfulness has gained a great deal of attention in the media in recent years. Due to this, participants have likely paid more attention to reports. This attention may serve as reminders to the participants and help them continue the practices they developed during the course. It may also be that by becoming more aware of one's thoughts through participation in the various lectures, discussions, and activities, the older adults in this study became more mindful. When asked about the strategies they continue to use many mentioned mindfulness techniques, changing perspectives about a situation, and purposively thinking to be more positive. This is evident by some of the thoughts expressed by the participants. For example, one of the participants discussed how just being present while washing their hands has helped them learn to be more present throughout their day. One of the directors has also indicated how she has observed people at the center are being more open to discussion and less judgmental.

Other participants provided examples of non-judging $(p<.05)$ or non-reactivity $(p<.05)$ with one discussing how they have learned to stop when they recognize they are starting to feel frustrated and then realize they have the power to change that emotion. Another participant stated how they used to feel they were less happy than most other people; however, now they recognize that it is all about how they are perceiving things and they now have the power to change those perceptions.

One of the center directors mentioned that she has heard participants in the class use language and techniques learned from the Art of Happiness and they have encouraged others to take a moment to think about what just happened and take a moment before reacting to it. This director, who is very involved in her center, has observed this type of discussion which indicates continued application of the principles taught in the course still being applied in their daily lives.

Another potential outcome of the program could be related to having built a better sense of community. The participants in the class became close with each other through sharing their experiences and thoughts on the various topics. This may have expanded to others in the senior center community. One of the senior center directors observed that they noticed how those in the class were having an impact on the overall environment of the center and had been discussing the lessons with others who had not chosen to be part of the class. She felt that these lessons were 
therefore being learned by many at the center and not just those who attended the classes. This may have helped to increase social connections in the older adults, which is an important component for their happiness [53].

There was a statistically significant effect of time on the negative affect of mood, tiredness. We know the literature suggests positive affect is associated with happiness [25] so is it possible decreases in negative affect such as tiredness may be linked to an increase in happiness. The negative mood affect of tiredness may also be more prevalent in these older adults. It is possible that being happy could decrease this negative mood affect.

Further, while forgiveness was not measured as a construct, it can have a large impact on one's happiness $[2,54,55]$. An example of this was shared by one of the directors. She shared that after one of the participants wrote a letter of forgiveness to her sister who lives over 1500 miles away and to whom she had not spoken in over 35 years was able to reconnect. They are now communicating and much happier to have put this past behind them.

The major limitation of this study is there was not a randomized control trial and there was no attempt to compare to a control group of independently living older adults. This program was also conducted in senior centers, which are community centers for older adults and was a quasiexperimental study. Therefore, further researched is needed to test the widespread implementation in senior center /community center settings. Additionally, we did not measure the impact that this program may have had on the participants in the senior center via social support or simply meeting in groups for lecture, discussions, and activities. Although we did recruit from two different senior centers in ethnically diverse communities, this sample was not ethnically diverse and was primarily comprised of females so it is difficult to generalize the findings to older adult males or other ethnic populations. Lastly, while Buddhist philosophy was a basis for some of the activities and discussions only traditional Western concepts were measured.

\section{Conclusions}

This study shows promise in the course being able to have some long-term impact on older adult's perceptions and psychological health and well-being. Of course, it also demonstrated how important booster sessions and reminders are to create long term behavior change. Changing how older adults react to situations, stressors, and their perception of things is similar to trying to impact any other aspect of their life such as diet or exercise. Habits do not change easily and once that daily reminder is removed people tend to gravitate back to the way they have always been. As the saying goes, "use it or lose it". This was apparent in this study as some of the measures that were significant immediately after the course were not significant 6 months later. We would like to add other follow-ups to the intervention where participants will receive weekly reminders, activities (new and old), and an opportunity to ask questions of the researchers.

The course also seemed to have help develop a positive sense of community thereby making the center an attractive place that may encourage greater membership. The success of the course further illustrates how neuroplasticity in older adults is still there and we can continue to learn throughout our healthy life. This also demonstrates the potential for positive reception of positive psychology programs in a population of older adults. It may be that focusing on positive aspects of mental well-being is a better avenue to approach mental health in a population of older adults who are often faced with life events or situations which can impact them. With proper training 
and facilitation, the "Art of Happiness" is one program that can be effectively implemented in any community center. We believe this approach can be effective across cultures, religious, gender, and ethnic groups. Further research is needed to bear this out, but the current findings show promise.

\section{Acknowledgments}

We would like to acknowledge the staff and members of Howard Weston Senior Center and Claymore Senior Center.

\section{Author Contributions}

Orsega-Smith performed the initial analysis and wrote the drafts of the paper; Goodwin conceptualized the study and wrote the discussion; Turner, Greenawalt, \& Rathie conducted the intervention at the senior center. They also assisted in writing the introduction; Ziegler completed the final analysis.

\section{Funding}

University of Delaware Summer Service Learning partially funded this project.

\section{Competing Interests}

The authors have declared that no competing interests exist.

\section{References}

1. Lama D, Cutler HC. The Art of Happiness: A handbook for living. New York; Riverhead Books. 1998.

2. Lyubomirsky S. The how of happiness: A scientific approach to getting the life you want. New York: The Penguin Press; 2008 (p.20-22).

3. Turner J, Greenawalt K, Goodwin S, Rathie E, Orsega-Smith E. The development and implementation of the art of happiness intervention for community dwelling older adults. J Educ Gerontol. 2017; 42: 630-640.

4. Administration on Aging, U.S. Department of Health \& Human Services. A Profile of Older Americans: 2015. 2015.

5. Centers for Disease Control and Prevention. The State of Aging and Health in America 2013.Retrievedfrom

http://www.cdc.gov/features/agingandhealth/State_of_aging_and_health_in_america_2013. pdf

6. Baird BM, Lucas RE, Donnellan MB. Life satisfaction across the lifespan: Findings from two nationally representative panel studies. Soc Indic Res. 2010; 99: 183-203.

7. Bonhote K, Romano-Egan J, Cornwell C. Altruism and creative expression in a long-term older adult psychotherapy group. Issues Ment Health Nurs. 1999; 20: 603-617.

8. Shapira N, Barak A, Gal I. Promoting older adults' well-being through Internet training and use. Aging Ment Health. 2007; 11: 477-484. 
9. Shtompel N, Whiteman K, Ruggiano N. Negative feelings and help seeking among older adults with chronic conditions. J Gerontol Soc Work. 2014; 57: 810-824.

10. Steptoe A, Deaton A, Stone AA. Subjective wellbeing, health, and ageing. The Lancet. 2015; 385: 640-648.

11. Corcoran J, Brown E, Davis M, Pineda M, Kadolph J, Bell H. Depression in older adults: a metasynthesis. J Gerontol Soc Work. 2013; 56: 509-534.

12. Centers for Disease Control and Prevention and National Association of Chronic Disease. The State of Mental Health and Aging in America Issue Brief 1: What Do the Data Tell Us? Atlanta, GA. 2008.

13. Blazer DG. Depression in late life: review and commentary. J Gerontol A Biol Sci Med Sci. 2003; 58: 249-265.

14. National Council on Aging. Higher tier evidence -based health promotion/disease prevention programs. 2017. Retrieved from: https://www.ncoa.org/resources/ebpchart/.

15. Baltes PB, Baltes MM. Psychological perspectives on successful aging: The model of selective optimization with compensation. Successful Aging: Perspectives from the Behavioral Sciences. 1990; 1: 1-34.

16. Duckworth AL, Steen TA, Seligman ME. Positive psychology in clinical practice. Ann Rev Clin Psychol. 2005; 1: 629-651.

17. Friedman E, Ruini C, Foy R, Jaros L, Sampson H, Ryff C. Lighten UP! A community -based group intervention to promote psychological well-being in older adults. Aging Ment Health. 2017; 21: 199-205.

18. Wood AM, Tarrier N. Positive clinical psychology: a new vision and strategy for integrated research and practice. Clin Psychol Rev. 2010; 30: 819-829.

19. Sutipan $P$, Intarakamhang $U$, Macaskill $A$. The impact of a positive psychological interventions on well-being in healthy elderly people. J Happiness Stud. 2017; 18: 269-291.

20. Seligman ME, Csikszentmihalyi M. Positive psychology. Am Psychol. 2000; 1: 5-183.

21. Oddone CG, Hybels CF, McQuoid DR, Steffens DC. Social support modifies the relationship between personality and depressive symptoms in older adults. Am J Geriatr Psychiat. 2011; 19: 123-131.

22. Seligman ME, Steen TA, Park N, Peterson C. Positive psychology progress: empirical validation of interventions. Am Psychol. 2005; 60: 410-421.

23. Kim ES, Park N, Peterson C. Dispositional optimism protects older adults from stroke: The health and retirement study. Stroke. 2011; 42: 2855-2859.

24. Ho HC, Yeung DY, Kwok SY. Development and evaluation of the positive psychology intervention for older adults. J Positive Psychol. 2014; 9: 187-197.

25. Lyubomirsky S, King L, Diener E. The benefits of frequent positive affect: Does happiness lead to success? Psychol Bull. 2005; 131: 803-855.

26. Davidson KW, Mostofsky E, Whang W. Don't worry, be happy: positive affect and reduced 10year incident coronary heart disease: The Canadian Nova Scotia Health Survey. Eur Heart J. 2010; 31: 1065-1070.

27. Steptoe A, Wardle J, Marmot M. Positive affect and health-related neuroendocrine, cardiovascular, and inflammatory processes. P Natl Acad Sci USA. 2005; 102: 6508-6512.

28. Cohen S, Doyle WJ, Turner R, Alper CM, Skoner DP. Sociability and susceptibility to the common cold. Psychol Sci. 2003; 14: 389-395. 
29. Ostir GV, Markides KS, Peek MK, Goodwin JS. The association between emotional well-being and the incidence of stroke in older adults. Psychosom Med. 2001; 63: 210-215.

30. Danner DD, Snowdon DA, Friesen WV. Positive emotions in early life and longevity: findings from the nun study. J Personality Soc Psychol. 2001; 80: 804.

31. Steptoe A, Wardle J. Positive affect measured using ecological momentary assessment and survival in older men and women. P Natl Acad Sci. 2011; 108: 18244-18248.

32. Zautra AJ, Johnson LM, Davis MC. Positive affect as a source of resilience for women in chronic pain. J Consulting Clin Psychol. 2005; 73: 212.

33. Kabat-Zinn J. Coming to our senses: Healing ourselves and the world through mindfulness. United Kingdom: Hachette Publishers; 2005.

34. Foulk MA, Ingersoll-Dayton B, Kavanagh J, Robinson E, Kales HC. Mindfulness-based cognitive therapy with older adults: An exploratory study. J Gerontol Soc Work. 2014; 57: 498-520.

35. Morone NE, Lynch CS, Greco CM, Tindle HA, Weiner DK. "I felt like a new person." The effects of mindfulness meditation on older adults with chronic pain: qualitative narrative analysis of diary entries. J Pain. 2008; 9: 841-848.

36. Carter C, Forgive and Feel Happier, May 12, 2008. Available from: https://greatergood.berkeley.edu/article/item/forgive_andfeel_happier. Accessed August 29, 2018.

37. Leung A, Kier C, Fung T, Fung L, Sproule R. Searching for happiness: The importance of social capital. In: Delle Fave A. (eds) The exploration of happiness. Happiness studies book series. Dordrecht: Springer, 2013 (p.247-267).

38. Amado-Boccara I, Donnet D, Olie JP. Conception of mood in psychology. Encephale. 1993; 19: 117-122.

39. Watson D, Clark LA, Tellegen A. Development and validation of brief measures of positive and negative affect: the PANAS scales. J Personality Soc Psychol. 1988; 54: 1063-1070.

40. Clark LA, Watson D, Leeka J. Diurnal variation in the positive affects. Motivation Emot.1989; 13: 205-234.

41. Berry DS, Hansen JS. Positive affect, negative affect, and social interaction. J Personality Soc Psychol.1996; 71: 796-809.

42. Seligman ME. Flourish: A visionary new understanding of happiness and well-being. New York: Simon and Schuster; 2012.

43. Lyubomirsky S, Lepper HS. A measure of subjective happiness: Preliminary reliability and construct validation. Soc Indic Res. 1999; 46: 137-155.

44. Baer RA, Smith GT, Hopkins J, Krietemeyer J, Toney L. Using self- report assessment methods to explore facets of mindfulness. Assessment. 2006; 13: 27- 45.

45. Diener ED, Emmons RA, Larsen RJ, Griffin S. The satisfaction with life scale. J Personality Assess. 1985; 49: 71-75.

46. Pavot W, Diener E. The satisfaction with life scale and the emerging construct of life satisfaction. J Positive Psychol. 2008; 3: 137-152.

47. Thayer RE. Measurement of activation through self-report. Psychol Rep. 1967; 20: 663-678.

48. Thayer RE. The biopsychology of mood and arousal, New York: Oxford University Press. 1989.

49. Cohen S, Kamarck T, Mermelstein R. A global measure of perceived stress. J Health Soc Behav. 1983; 4: 385-396. 
50. Lee EH. Review of the psychometric evidence of the perceived stress scale. Asian Nurs Res. 2012; 6: 121-127.

51. Kroenke K, Strine TW, Spitzer RL, Williams JB, Berry JT, Mokdad AH. The PHQ-8 as a measure of current depression in the general population. J Affect Disorders. 2009; 114: 163-173.

52. Arroll B, Goodyear-Smith F, Crengle S, Gunn J, Ngaire K, Fishman T, et al. Validation of PHQ-2 and PHQ-9 to screen for major depression in the primary care population. Ann Fam Med. 2010; 8: 348-353.

53. Fowler JH, Christakis NA. Dynamic spread of happiness in a large social network: longitudinal analysis over 20 years in the Framingham Heart Study. Brit Med J. 2008; 337: a2338. https://doi.org/10.1136/bmj.a2338.

54. Chan DW. Subjective well-being of Hong Kong Chinese teachers: The contribution of gratitude, forgiveness, and the orientations to happiness. Teach Teach Educ. 2013; 32: 22-30.

55. Maltby J, Day L, Barber L. Forgiveness and happiness. The differing contexts of forgiveness using the distinction between hedonic and eudaimonic happiness. J Happiness Stud. 2005; 6: $1-13$.

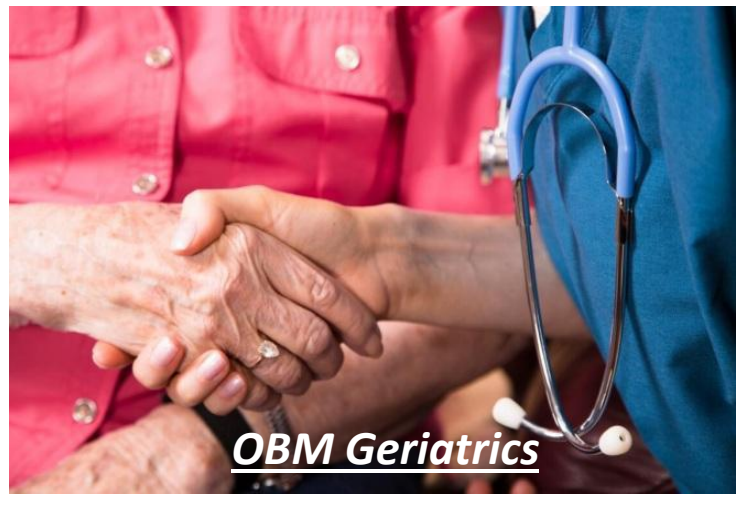

Enjoy OBM Geriatrics by:

1. Submitting a manuscript

2. Joining in volunteer reviewer bank

3. Joining Editorial Board

4. Guest editing a special issue

For more details, please visit: http://www.lidsen.com/journals/geriatrics 
Research Article

\title{
Acceptance of Information and Communication Technologies for Healthy and Active Aging: Results from Three Field Studies
}

Filomena Papa *, Bartolomeo Sapio, Enrico Nicolò

Fondazione Ugo Bordoni, Viale del Policlinico 147, 00161 Roma, Italy; E-Mails: fpapa@fub.it; bsapio@fub.it; nic@fub.it

* Correspondence: Filomena Papa; E-Mail: fpapa@fub.it

Academic Editor: Lisa Hollis-Sawyer

Special Issue: Got Aging? Examining Later-life Development from a Positive Aging Perspective

OBM Geriatrics

2019, volume 3 , issue 1

doi:10.21926/obm.geriatr.1901028
Received: October 31, 2018

Accepted: December 19, 2018

Published: January 04, 2019

\begin{abstract}
Background: Information and Communication Technologies (ICTs) have the potential to promote healthy aging and increase the quality of life of older adults. However, several barriers like access, performance, psychological, and privacy issues still exist against fully deploying ICT solutions for older adults. To determine useful methods to overcome such barriers, this work investigated the possible factors that prevent elders from directly accepting ICT services based on three field studies.

Methods: The Unified Theory of Acceptance of Use of Technology (UTAUT) was adopted as the general reference framework.

Results: Performance Expectancy (perceived usefulness) and Facilitating Conditions (provided user support) seem to be the most relevant factors in all field studies. Also, the relevance of Hedonic Motivation (pleasantness of use) emerges. The role of Effort Expectancy (ease of use) seems to be affected by the user profile, which includes education level, present technology skill, and employment status.
\end{abstract}

Conclusions: Overall, results confirm that the reference model variables represent the key factors to predict older adults' viewpoints toward ICT. The use of familiar devices (like TV sets) in the interaction with new technologies was determined to be a significant factor in

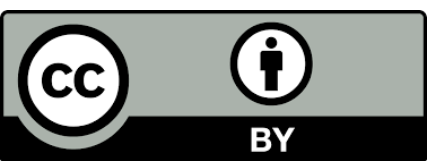

(C) 2019 by the author. This is an open access article distributed under the conditions of the Creative Commons by Attribution License, which permits unrestricted use, distribution, and reproduction in any medium or format, provided the original work is correctly cited. 
promoting ICT acceptance by older adults. Furthermore, acceptance/rejection of ICT services depends on classical socio-demographic variables and on participants' attitude towards technology, lifestyle changes, and the beliefs that individuals share with members of their social context.

\section{Keywords}

Effort expectancy; electronic health record; facilitating conditions; healthy aging; hedonic motivation; performance expectancy; technology acceptance; unified theory of acceptance and use of technology (UTAUT)

\section{Introduction}

Information and Communication Technologies (ICTs) can provide useful and effective tools to meet the expectations of people to live longer higher quality lives. Current technological trends in health care are focused on technological solutions to support disease management and medical records. ICTs may also enhance communication between participants using the technology, which plays a significant role in their social capabilities and mental well-being,

Designing ICTs for older adults requires specific understanding of their needs and comparative analysis of the generational use of new media and technology [1].

In a recent study by the Italian Statistics Institute (ISTAT) [2], statistics on Internet use show that $30.8 \%$ of people $65-74$ years old and $8.8 \%$ of people over 75 have used the Internet during the last year.

Several barriers like access, performance, psychological, and privacy issues still hinder the full deployment of ICT solutions for older adults [3]. In particular, rejection attitudes toward new ICT services and social media can arise, especially in groups with low income and poor education [4]. In order to overcome these barriers, the investigation of factors that affect older adults from accepting ICT services provides useful insight to identify adequate policies to promote acceptance. One of the most recognized models for innovative and emerging new technologies is the Unified Theory of Acceptance and Use of Technology (UTAUT), which is extensively used in various applications, such as technology use in different organisational contexts [5], consumers' use of technology, e-commerce. This model is comprised of different constructs, including intention to use ICT services, perceived usefulness, and perceived ease of using ICT services. Implementing the UTAUT model as a general reference framework, this paper discusses the factors affecting acceptance of ICT services by older adults. Results were obtained from three field studies, which focused on the acceptance by older adults through direct involvement with different ICT technologies (i.e., a TV-based technological solution for social interaction, an Electronic Health Record, Digital Television) and implemented the same reference framework.

\subsection{Field Study 1}

This study analyzed the use of ICTs to support social interactions of older adults which are defined in this case as $65+$ years of age. The virtual world via the Internet has potential to mitigate 
social isolation problems faced by seniors. This work presents a qualitative investigation of the reactions, opinions, and sentiments about a TV-based technological solution to promote social interactions of less educated older adults (i.e., older adults unskilled in ICT use with low education and low income). Experimental data were collected by extensive trials involving 40 end users in the European AAL project EasyReach. A methodology called "scenario engagement" was applied to engage participants in a live demonstration, which was mediated by a facilitator who assisted older adults to interact with the system.

\subsection{Field Study 2}

This study relates to the use of ICT solutions to maintain the physical well-being of older adults, to promote the use of self-monitoring technologies for patients with chronic illnesses, and to better prevent the loss of independence and good health. The acceptance by citizens of one of the most advanced implementations of the Electronic Health Record (EHR) in Italy is investigated in this study. The system provides applications for self-measurement, inputting drug treatments, entering examinations conducted at laboratories, downloading and viewing medical reports as soon as they are issued, online payments, virtual prescriptions. The UTAUT model and its extension UTAUT2 were adopted as general reference frameworks. The quantitative investigation was conducted using the CAWI (Computer Assisted Web Interview) method, with a total number of 15,102 users completing the web interview. Among these citizens, 5,664 were over 55 years old.

\subsection{Field Study 3}

This field study explores the interaction of older adults with new television services and new ICT services. TV services are a central source for elders and could contribute to maintaining their mental well-being. Furthermore, the use of familiar devices (like TV sets) combined with ICT services could promote the adoption of these services by older adults. This qualitative study performed in Italy is focused on the acceptance of Digital Terrestrial Television (DTT) after the analogue switch-off, that is the transition from analogue to digital terrestrial television. In order to identify the main predictors of digital television usage by older adults, the UTAUT model was applied, followed by a focus group discussion analysis.

In Section 2, the adopted reference frameworks are described (UTAUT and UTAUT2). In Sections 3-5 the three field studies are presented, and Section 6 discusses the factors affecting acceptance of ICT services by older adults.

\section{The Reference Framework}

From the point of view of the ICT users, the decision to accept new technologies is an innovative step and is based on two conditions: if the technology is useful to them, and if they have the skills, means, and situation to use the presented technology. Some reasons for innovative change include the natural curiosity to try new things and social influence by significant others who push them to innovation, such as family, friends, or bosses [6]. Understanding the social dynamics of ICT might help engineers to focus technological designs on meeting the users' demands. Previous studies have reported the role of domestication of Information and Communication Technologies in depth and its influence on usage by elders $[7,8]$. 
User experience is a very broad concept dealing with all aspects of a user's interaction with the product/service, including how it is perceived, learned, and used [9]. Behind the user experience concept, there is a holistic approach aimed at balancing the pragmatic aspects mainly related to the execution of the task- and non-task-related aspects. Examples of these include aesthetic, psychological, and emotional aspects like self-expression through technology use [10].

The evaluation of different user experience aspects can be utilized as input for user acceptance models. These models are powerful tools to explain individual acceptance and usage of a technological system. They also allow the identification of most relevant factors that facilitate and hamper usage (see, for instance, [11]).

The Unified Theory of Acceptance and Use of Technology (UTAUT) [5] is one of the most recognized acceptance models. This model, comprising different aspects of usage behavior, has been extensively implemented to explain the adoption and use of various innovative ICT products and services.

The UTAUT can explain usage behavior of an innovative technology or service starting from the central role of behavioral intention. The key dependent variable in the UTAUT model is the intention or the use of technology; hence, the primary purpose of the model is to understand and provide an explanation for the phenomenon "use of information technology" as a dependent variable. The role of intention, as an antecedent of behavior, is a critical factor in the model. In the formulation of the UTAUT model, four constructs are identified, which may play a significant role as direct determinants of user acceptance and usage behavior:

- Performance Expectancy is the degree to which an individual believes that using the system will help him/her to attain gains in job performance.

- Effort Expectancy is the degree of ease associated with the use of the system, which is strongly connected to the degree to which a person believes that using the system would be free of effort.

- Facilitating Conditions are related to the degree to which an individual believes that an organization or technical infrastructure exists to support the use of the system.

- Social Influence is the degree to which an individual perceives that significant others, such as spouses and family members, believe they should use the new system.

In the specific case of technology designed for an aging population, it is possible to measure the above-mentioned constructs using some of the following indicators:

Performance Expectancy (perceived usefulness of services):

- Degree of interest of the user towards provided services and content;

- Update of provided content;

- Service interaction and fruition modalities;

- Service capability to attract and entertain the user, avoiding banality and tediousness in the interaction;

- Satisfaction of using service in comparison to the same kind of service delivered through other channels.

Effort Expectancy (perceived ease of use): 
- Ease of access to the services;

- Ease of system use;

- Ease of navigation;

- Perception by the users of possible system dysfunctions (excessive waiting times, unclear costs, etc.).

Social Influence:

- Attitude towards technology of the persons who are considered important by the user (e.g., affective and charismatic leaders);

- Behavior towards technology of own reference groups (e.g., friends, associations).

Facilitating Conditions (provided user support):

- User manuals;

- Call center or contact center.

Variables, such as age, gender, experience, and voluntariness of use, are important moderators included in the UTAUT model. Income and education have been proposed as moderators to be validated in future studies [11].

More recently, the UTAUT2 model was proposed as an extension of the UTAUT model. UTAUT2 can be utilized in larger contexts and includes new constructs connected to habit, price value, and hedonic motivation in use [12].

Furthermore, in the particular case of e-health, it is necessary to consider an additional construct [13] concerning security perception, privacy protection, and personal data confidentiality.

\section{Field Study 1: TV Set to Overcome Barriers for Older Adults in ICT Use}

Assuming that the ability to use a TV remote control can be exploited as a sufficient factor to become effective users of new digital social environments and to reduce rejection attitudes, a qualitative investigation on the reactions of older adults toward a TV-based technological solution to promote social interaction was carried out. The investigation was developed by the Easyeach Project (AAL-2009-2-117) under the EU Seventh Framework Program (Ambient Assisted Living Joint Program, AAL Call 2). A technological solution was implemented, which aimed at fostering social interaction for individuals within the aging population who are resistant to the adoption of new technology or lack the knowledge and skills needed to use social networks. A special interactive environment was created to enhance the social contact of aged people and to respond to their needs by using a "traditional" technology that does not introduce any further resistance to the acceptance of the innovation.

The user-centered design approach [14] was adopted to directly involve less educated older adults (unskilled in ICT use, low education, and low income) in the early stages of the development process. A pilot investigation was carried out in Rome, Italy, involving real users [15].

The use of ICTs in health care could allow disadvantaged groups of citizens, such as elderly, chronically ill people, or those living with various physical and cognitive disabilities, to maintain 
vital and rich connections with families, friends, and careers. ICTs can help them keep in touch and socialize through social network applications [3].

The field of studies related to Social Network Sites (SNS) concerns the use of ICTs to support social interactions of people through SNSs. The virtual world and social networks are potentially important to meet elderly persons' needs, who can subsequently create and develop relationships with other older adults and members of different backgrounds and generations. SNSs have the potential to mitigate social isolation problems felt by seniors and to help them maintain their social networks [16].

Despite the many potential benefits for elderly socialization, a base core of knowledge and skills is needed to use social networks, which is not widespread in the community of less-educated older adults. Few studies have been developed in the area of socialization applications for unskilled older adults. For example, an investigation [17] developed in the framework of the European AAL research program utilized a Smart TV platform to support social interaction of older adults with their relatives and other people sharing the same interests. The approach was to use well-known (by older adults) existing technology to avoid rejection attitudes and to provide a solution to mitigate social isolation of older adults. Following a similar approach, another study [18] revealed that when technology is embedded in everyday objects, such as a TV set, older people can use it easily.

\subsection{The EasyReach Solution}

The main hypothesis in designing the EasyReach solution was that for less-educated aged people, who do not possess the necessary skills to use the Internet and social networks, daily experience with TV and remote control could be a key qualification to become an effective user of those services. Moreover, a TV-like system may help overcome the psychological barriers and the rejection attitudes toward new ICT services and social media. In comparison with the abovementioned studies, using similar approaches, the EasyReach technological solution utilizes a new kind of remote control that combines gesture recognition with video and audio capture capabilities.

The key idea of EasyReach is to maintain simplicity for users, in both system installation and utilization. It is based on a special social TV channel accessed by older adults through their own TV set, a set-top box and a specialized remote control unit endowed with gesture recognition and video and audio capture capabilities [19]. A hidden personal assistant (PA) elaborates user preferences in the background, allowing better focalization on his or her social interests.

After connecting the set-top box to the TV set, the user is immediately able to join the social channel and enjoy the benefits of networking.

With the EasyReach special remote control, the user can take pictures and videos of special events (e.g., a family dinner, a birthday party, etc.), share his or her knowledge and experience by creating a video message and posting it on thematic groups, and simply have conversations with friends and relatives. The remote control's gesture recognition mechanism helps the user navigate by giving him/her the possibility to scroll a personal list of users and groups through simple gestures.

Moreover, the system allows organizations and official institutions to join the social channel in order to be easily reachable by the users, send them useful information/advice, and help them 
more directly. The opportunity to make the user's own expertise available to the whole EasyReach community can be invaluable to maintain the self-perception of being socially active.

\subsection{Method}

The main requirements about elderly participants as users can be shortly summarized into factors: "poor education," "ICT unfamiliar," "low income," and "resistant to change." These four factors strongly influence the technical solutions and the way to select the potential participants. In this work the research team analyzed different possible alternatives, especially in view of the direct involvement of users in field trials and their reactions toward the proposed technical solution. Two senior centers in Rome were selected [15]. Preliminary sessions to better understand end users' reactions and behaviors were purposely organized. These early trials quickly revealed many unpredictable aspects. It was recognized that applying any quantitative metrics to collect data was an impossible task, mostly due to participants' resistance and ungovernability during the sessions. The early sessions also helped design a pilot system capable of collecting effective users' reactions, opinions, and sentiments about the EasyReach system, including ease of use and usefulness. User experience and acceptance models were taken as broad references.

Laboratory testing revealed a lack of fluidity in interaction with the system, resulting in a frustrating experience for users. Because gestures to browse the interface were not very easy to learn, the project team decided to set up a sort of "mediated experience" for the older adults during the pilot using an original methodology called "scenario engagement" [15]. This approach takes into account the strong support needed by older adults and their interaction with different technologies [20]. The choice was to analyze user testing [21], in which participants engaged in a live demonstration of the EasyReach solution with the support of a facilitator.

A "scenario engagement" methodology for the execution of the field evaluation is defined as the intent of carrying out an assessment and the "acceptance towards the idea of..." or the "what if..." This methodology was implemented after the involved older adults were engaged in a live demonstration illustrating scenarios of possible use.

Implementing scenario engagement, a live demonstration performed under certain procedural settings can be equally effective to assess the users' reactions and their own experiences in interacting with the system. Even though the users can control the system, they are guided stepby-step by a mediator supporting them in the manipulation of devices.

\subsubsection{Participants}

A screening questionnaire was administered to select participants for the qualitative investigation.

Two senior centers, representing two different geographical and cultural areas in Rome, were selected for the pilot test in cooperation with Federazione Nazionale Pensionati (FNP), the retired and elderly federation of CISL (trade union).

Presentation meetings with older adults were organized in each of the two senior centers in September 2012. During these meetings, the screening questionnaire was administered to participants. 
The criteria used in the selection process were related to age (preferably in the range 65-80), education (preferably "primary" and "secondary" education), and computer and Internet usage (preferably no more than once per month). Physical characteristics of participants, such as visual and hearing capabilities, were not considered in the selection.

Forty participants ( 21 males, 19 females) were recruited for the pilot test (30 individuals from the two senior centers, 15 for each center, plus 10 others selected from FNP-CISL as "privileged witnesses" of the elderly world).

Demographic data of participants showed a balanced distribution of genders $(52 \%$ males and $48 \%$ females) with a peak age range of 66-70 (32\% of total participants). Most of them had a low level of education ( $80 \%$ in the "primary - secondary" education range). Almost all participants did not use computers (85\%) or the Internet $(72 \%)$.

\subsubsection{Procedure}

The field trial was realized by setting up group sessions at the senior center premises involving the selected users. About 10 users were involved in each session. A total of four group sessions were realized with two sessions at each senior center in 2013.

Each session was developed through three main segments: a demonstration of the main features of the EasyReach system (remote control, user interface); scenario engagement of the older adults in tasks with the EasyReach prototype, such as planning a group activity (a "mediated user experience" was setup); and collection of the users' opinions. The duration of the overall group session was about 80 min.

The usage scenarios were carefully rehearsed to avoid time consuming or likely-to-fail actions. User profiles and user groups identified in the scenarios were created in the set-top boxes, and good quality videos and photos were uploaded offline into the "galleries" of selected users.

During the trial sessions, two simple activities were identified, which fit the usual life habits of participants from the two senior centers. Two different scenarios were demonstrated, showing the added value of EasyReach utilization in comparison to traditional communication media (e.g., phone calls).

The theme of the first scenario engagement was the organization of a group activity: "A Day to The Cinema." The theme of the second scenario was the communication of good news to friends: "Becoming a Grandmother."

One researcher conducted the session using a printed script, while a second researcher (facilitator) interacted with the system during the group session. Another researcher (recorder) took note of the most relevant issues emerging from the interview.

User opinions were collected, which concerned the following issues: perception of usefulness, pleasantness of use, and ease of use of the EasyReach solution; intention of future service usage in different environments (at home and at the senior center) when system is available; and the potential for social inclusion and improvement in quality of life.

General impressions about the system, its main features/service, and suggestions for improvement were also investigated.

In the last part of the session, a group interview was realized to collect user opinions about the EasyReach system. 
In addition to the group interview, personal interviews were administered to 10 "privileged witnesses" to analyze the potential of the EasyReach solution.

Both group and personal interviews were audio and video recorded. Transcriptions of group and personal interviews were made, then content analysis was conducted to point out the most relevant emerging topics.

\subsubsection{Ethical Considerations}

Although a formal ethical approval was not explicitly required by the AAL program, a targeted analysis of the ethical issues was included in the EasyReach work plan. After the preparation phase of the pilots, the following aspects were highlighted: privacy protection and confidentiality, informed consent/disclaimers, and risk assessment. Formal discussions taken inside the consortium produced a general agreement about the policy and ethical guidelines to be observed before, during, and after the pilots. Both the EasyReach meeting notes (Project Steering Committee, Technical Management Board) and Deliverable D6.1 "Detailed Plan for the Pilots" [22] provide proof of the work developed on the matter.

In particular, the executive team of the Rome pilot included two extra researchers who supervised the application of ethical guidelines.

The EasyReach work plan approved by the AAL program, as required, included an Ethical Issues Table. In this table, it was stated that the research involves "adult healthy volunteers" and that "all country specific provisions, specific laws and regulations will be respected." Also in the Rome pilot, the regulations for personal data protection (law no. 186, 30 June 2003) were respected. A formal ethical approval by a recognized authority was not explicitly required by the AAL program for funding this research.

As stated in the EasyReach work plan, informed consents were submitted to end users in advance. They were informed of the aim to study that specific population, the voluntariness in the participation, the possibility to withdraw at any moment, the policy for anonymity, the expected benefits/risks of this research, how long the confidential information would be kept, and the indication of a reference person to contact for any questions related to the research.

Each participant in the Rome pilot was requested to compile a disclaimer (i.e., a "release form for media recording"), in which the participant was informed that "the experimental activities and discussions will be recorded and some photos will be taken." In this form, the participants were asked to express the "agreement for the diffusion of the audio and video materials obtained during the sessions through different channels such as scientific papers, conferences, TV programs, internet, etc." All participants in the Rome pilot signed the above disclaimer as requested and returned the signed form to the research team.

\subsubsection{Data Analysis}

The user acceptance UTAUT model was adopted as the theoretical framework for investigation and data analysis [5] in association with the concepts of quality of life and perceived loneliness.

In the investigation, the perception of improved quality of life was included in the section of the UTAUT model related to the perception of usefulness of the system. We focused the group interviews on themes related to perceived usefulness (Performance Expectancy), perceived pleasantness and ease of use (Effort Expectancy), and intention of use (Behavioral Intention). The 
Facilitating Conditions in this investigation were part of the scenario engagement methodology, thus we decided not to investigate this issue. We decided not to investigate social influence since this theme was a sensitive topic to discuss with this particular class of older adults.

From the group interviews, about $100 \mathrm{~min}$ of video/audio registrations were obtained.

From the personal interviews to "privileged witnesses," about $300 \mathrm{~min}$ of video/audio registrations were obtained. Qualitative content analysis was executed using both notes and transcriptions.

This analysis was developed by researcher members of the team through three steps: (1) reading notes and transcriptions, (2) identifying relevant opinions/sentences, and (3) making comments. The results were consolidated in group discussions involving all members of the research team. More details about data analysis procedures can be found in [15].

\subsection{Results}

The investigated solution can be effectively employed to foster social interaction, particularly when it is introduced in a collective use environment (e.g., senior center). Although the focus of the investigation was on information and communication technology-enabled social environments, the end users themselves strongly suggested that future systems include extra functions, as essential opportunities for their potential digital lives, meaning medical or health services and bridges toward Public Administrations.

The pleasantness and ease of use were also carefully examined as potential factors that may affect usage. In general, the system was considered to be simple and pleasant to use, except for the dislike of the specific remote control.

The intention to use the system as a tool to enhance social communication was also investigated. A wide range of usage intentions was gathered which span from negative to neutral to enthusiastic attitudes. Future suitable scenarios of use for this solution are likely to favor the collective environment (at the senior center) rather than a solitary use from home. Technologyresistant older adults asked for strong support and help, from which an environment allowing a collective use seems to be advisable.

Despite the difficulties in direct interaction (i.e., slowness and inadequacy of the remote control), personal interviews to "privileged witnesses" showed great potential for social inclusion and the great capacity of EasyReach to improve the quality of life of aging people. The system seemed to stimulate the participants' curiosity as well to open their mental horizons to further possibilities that can facilitate their daily lives.

\section{Field Study 2: Older Adults' Acceptance of e-Health Services}

\subsection{Context}

This study introduces a quantitative investigation about the acceptance by citizens of one of the most advanced implementations of the Electronic Health Record (EHR) in Italy. From a national perspective, the EHR initiative aims at improving all care activities throughout the patient's life. Therefore, the dossier is continually fed by professionals within the Italian National Health Service (SSN) and regional services, as well as at the request of the citizens with the medical records held by them. 
The improvement of health services and care activities is perceived very important by older adults. Medical or health related services have high recurrence among elderly needs and requirements to increase their quality of life [15]. To satisfy the needs of older adults the EHR could provide potential benefits [23].

The regional EHR presented here, TreC service (literally, Citizens' Clinic Record), is promoted by the Trento Autonomous Province in Italy.

This exploratory study allows researchers to investigate factors that facilitate or hamper the acceptance of TreC services by citizens and to compare the results obtained for the overall sample of citizens containing the group of adults over 55. At the same time, it can provide useful input to define specific public policies to promote acceptance of innovative e-health solutions by older adults.

\subsection{Method}

\subsubsection{The TreC System}

The system was designed to be a configurable platform of applications and initially included a series of functions for archiving and codifying healthcare data and applications for selfmeasurement, inputting drug treatments, entering examinations conducted at laboratories outside the Region, and so on. In addition, from the outset, the infrastructure offered the opportunity to download and view medical reports as soon as they were issued by laboratories. Later, other services, such as online payments, virtual prescriptions and other minor services were made available [24].

\subsubsection{Data Collection and Participants}

From the time of its birth, the TreC service was monitored by means of quantitative and qualitative studies planned and developed by the Department of Sociology and Social Research of the University of Trento for the entire duration of the project (2008-2013).

This investigation is based on one activity in particular, a CAWI (Computer Assisted Web Interview) addressed to approximately 34,000 active users registered with the infrastructure at the end of the fourth year of operation (2016).

A specific section of the CAWI was finalized to measure acceptance constructs (see Section "Investigated issues").

A total number of 15,102 users completed the web interview, 5,664 of which were over 55 years old. Participants belonged to different classes of gender, age, education, and employment. Overall, the average participant profile was of middle age, high education (degree or post degree), skilled in technology use, and employed [25].

\subsubsection{Investigated Issues}

To investigate the factors affecting acceptance by citizens of the TreC system, the UTAUT2 model (see Section 2) was used as a broad reference. In the case of e-health and electronic health records, we considered all four constructs of the original UTAUT model (Performance Expectancy, Effort Expectancy, Social Influence, and Facilitating Conditions) and Hedonic Motivation from 
UTAUT2. Price Value was not considered because TreC users did not pay for the service, and Habit was also excluded due to the limited experience users may have with the system. The additional construct concerning Security Perception, connected to perception of privacy protection and personal data confidentiality provided by the TreC system, was included.

As described in Section 2, the construct "Behavioral Intention" was adopted to measure acceptance.

The items included in the questionnaire to measure each construct are summarized in Table 1.

Table 1 Items of the questionnaire

\begin{tabular}{ll}
\hline Construct & Item \\
\hline Performance Expectancy (PE) & PE1 The TreC system is useful in my every day life \\
& PE2 The TreC system helps me to manage my health \\
& PE3 The TreC system allows me to save time \\
Effort Expectancy (EE) & EE1 Learning how to use TreC is easy for me \\
& EE2 After the first accesses, TreC is easy to use \\
Perceived Security (PS) & PS1 TreC protects confidentiality of my personal data \\
Social Influence (SI) & SI1 My relatives/friends think that it is useful for me to use \\
Hedonic Motivation (HM) & TreC \\
Facilitating Conditions (FC) & FC1 I have the technological resources to use TreC \\
& FC2 I have the knowledge necessary to use TreC \\
& FC3 I can get the necessary help when I have difficulties using \\
& TreC \\
Behavioral Intention (BI) & BI1 I intend to continue using TreC in the future \\
& BI2 I would like to continue using TreC more and more
\end{tabular}

All items were measured using a four-point Likert scale with the anchors being "strongly disagree" and "strongly agree."

\subsubsection{Ethical Considerations}

The ethical approval must be requested for all researches foreseeing experiments involving human beings, which imply a risk for the psychological and physical well-being of the participants. As was not the case for this research program, researchers applied the art. 110 of Italian Privacy Law (DL 186/2003 - Privacy in medical, Biomedical and epidemiology research). The invitation reported that the participation in the study was voluntary. Participants were clearly informed of the aim to study the specific user's population, voluntariness in the participation, possibility to withdraw at any moment, policy for anonymity, expected benefits/risks of this research, and indication of two reference persons to contact for any questions related to the research. An email address was provided for any request of information or for any communication about the inclusion/non-inclusion in the list used for the research. 


\subsection{Results}

As the first step in data analysis [25], the reliability (internal consistency) of multi-item scales was evaluated. The Cronbach's Alpha values were all greater than 0.7 , suggesting that all adopted scales can be considered reliable.

In order to investigate the factors affecting acceptance, multiple linear regression analysis was adopted, using the assessment of Behavioral Intention (BI) as the dependent variable and the assessment of PE (Performance Expectancy), FC (Facilitating Conditions), HM (Hedonic Motivation), PS (Perceived Security), SI (Social Influence), and EE (Effort Expectancy) as predictors.

In Table 2 the results of the regression analysis are summarized.

Table 2 Results of the multiple linear regression analysis (depending variable: Behavioral Intention)

\begin{tabular}{l|lll} 
Predictors & Beta (standardized) & $t$ & $p$ \\
\hline PE (Performance Expectancy) & 0.260 & 33.152 & 0.000 \\
FC (Facilitating Conditions) & 0.226 & 28.438 & 0.000 \\
HM (Hedonic Motivation) & 0.184 & 21.711 & 0.000 \\
PS (Perceived Security) & 0.102 & 14.675 & 0.000 \\
SI (Social Influence) & 0.076 & 10.247 & 0.000 \\
EE (Effort Expectancy) & 0.016 & 2.014 & 0.044
\end{tabular}

$\mathrm{N}=15102$, Overall model: $\mathrm{R}$-Square $=0.415, \mathrm{p}<0.044$

The regression model explains $41.5 \%$ of the variance in the intention to use TreC.

As shown in Table 2, all coefficients were significant ( $p<0.000$ for PE, FC, HM, PS and SI; $p<0.044$ for EE). The positive dependencies emerging from the analysis indicate that the more subjects perceived high values of these predictors, the more positive the evaluation of the intention was to use TreC in the future. Performance Expectancy and Facilitating Conditions (Beta=0.260 for PE and Beta $=0.226$ for $\mathrm{FC}$ ) appear to be the most relevant determinants of the Behavioral Intention to use the TreC system.

Other predictors of the intention to use TreC are Hedonic Motivation (Beta=0.181) and Social Influence (Beta=0.076). Hedonic Motivation is mainly related to the pleasantness of using TreC. Social Influence is connected to the social context of the user (opinions of relatives and friends).

In predicting $\mathrm{Bl}$, Effort Expectancy (Beta $=0.016$ for $\mathrm{EE}$ ) appeared to be of minor importance.

In Table 3 the results of the multiple regression analysis for the sample of adults over 55 are shown.

In summary, all predictors are significant with the exceptions of Social Influence and Effort Expectancy. The model does not have any integration by these predictors, which is probably a consequence of the recruitment. 
Table 3 Multiple linear regression analysis (depending variable: Behavioral Intention): adults $55+$

\begin{tabular}{l|lll} 
Predictors & Beta (standardized) & $t$ & $p$ \\
\hline PE (Performance Expectancy) & 0.273 & 16.863 & 0.000 \\
FC (Facilitating Conditions) & 0.236 & 14.283 & 0.000 \\
HM (Hedonic Motivation) & 0.197 & 11.457 & 0.000 \\
PS (Perceived Security) & 0.116 & 7.618 & 0.000 \\
SI (Social Influence) & 0.028 & 1.700 & 0.089 \\
EE (Effort Expectancy) & 0.020 & 1.147 & 0.25 \\
$\mathrm{~N}=5664$, Overall model: R-Square $=0.392, \mathrm{p}<0.000$ & &
\end{tabular}

\section{Field Study 3: Older adults' Acceptance of Digital Television}

User experience during transition from analogue to digital terrestrial television was presented in [26], focusing on older adults (age range from 70 to 79).

We performed a qualitative investigation to explore and analyze user experience with digital television and, more in general, with future services made available by broadband ICTs.

The results showed that the impact of the change from analogue to digital television was very strong. The investigation suggested that older adults who extensively watch new digital television channels were the same using their explorative abilities over the Internet (reading online newspapers or looking for old friends on Facebook).

More details about the portion of the study regarding older adults are provided in the following sections.

\subsection{Context}

The analysis of the transition process to digital terrestrial television in several European countries shows that older adults are unequivocally part of the population that meets the greatest difficulties and problems in the management of Digital Terrestrial Television (DTT) [27].

Numerous studies have been conducted on the use of ICT by older people [28]. However, there are few systematic surveys of the user experience with DTT and the user experience of older people with digital TV services [11, 29].

The investigation was based on the direct involvement of older adults and was mainly carried out by using the focus group technique.

The investigation was developed in the framework of the project "Monitor DTT" (Monitoring user experience with DTT) carried out by Fondazione Ugo Bordoni for the Italian Ministry of Economic Development (Department of Communication).

In this survey, the following main aspects of the user experience were considered: 
- Need and use of DTT compared to other media (e.g., Internet, satellite, or mobile television);

- Context of use (situations of use, social dynamics, social and individual TV viewing, and multitasking);

- Interaction with DTT equipment (remote controls, technological integration, impact of the equipment at home, etc.);

- Perception of the advantages and usefulness of the DTT (for example, better image quality, more programs and content, pay TV);

- Expectations on DTT and future ICT services.

\subsection{Methodology}

A qualitative approach was adopted to explore the phenomenon and construct hypotheses to understand the user's "point of view," behaviors, emotions, representations, needs, and future needs [30].

For this survey, in-depth interviews and focus groups were carried out.

The project started in July 2010 by interviewing two experts in the field external to the research team, in order to analize the issues to be explored during the focus groups.

After the interviews, a focus group was held in September 2010 with senior users (70-79 years). This focus group lasted two hours and consisted of 10 participants ( 5 males, 5 females).

A complete transcription of each focus group was performed, then content was analyzed to highlight the most relevant emerging topics.

\subsection{Results}

In the following, the main results are summarized for each UTAUT construct. The most significant quotations stated by the older adults in the study can be found in [26].

\subsubsection{Older Adults' Needs and TV Context of Use}

There is a feeling of melancholy, it is as if they were saying: "there is a gap that I have to fill". It is a sense of isolation, not so much real (presence of spouses, children, grandchildren...) as perceived, because they are now outside the work activity. This void is filled by the continuous presence of information (obtained mainly watching news TV programs) the link with the productive world, the "real" world.

The succession of the main news bulletins marks the day in its main moments, suggesting a regular routine. The information accompanies the day: in the morning people get up and turn on the TV to listen to the first news, they are updated at lunchtime, and then at dinner they watch the evening news.

If the need for information is the basis that unites participants, the choice of the preferred channel differentiates the cultural types present in the focus group, or the different channel demands. 
The most advanced range integrates the traditional/habitual reading of the daily newspaper on the Internet. At the opposite extreme are people who experience "nostalgia" when listening to the radio at any time or during any situation and who still live in a "classical" way (for these people, the modernization of the radio never happened). Then, there is the participant who "look on the world" and use satellite information to follow the news of other countries on CNN or BBC.

Television viewing takes place in different situations and within a specific social setting, which is most frequently the family.

Before the transition to DTT, television was watched in the family, within a circumscribed and known space, convenient, simple, and passive. When it was easier to get along, there was less choice and TV was watched together.

After the transition to DTT, television is watched alone, exploring new spaces, new channels in which one can also get lost. These are new viewing modalities separating the members of the family unit, bringing them to an enjoyment that is both individual and "scattered" among the various possible channels.

\subsubsection{Effort Expectancy}

Effort Expectancy in this case relates to the perceived ease of use of DTT and of the interaction through the remote controls.

The first answers to the questions of the interviewer on DTT can be described with the word "confusion." The main causes of this sense of confusion seem to be related to the interaction with remote controls. It is hard to find remote controls in the confusion of the different devices in the various rooms of the house.

There is even confusion about the existence of a "menu" that gives the possibility of receiving immediate information on programming. Participants are so confused such that someone thinks of the culinary sense of the word "menu."

As a result, the use of digital television requires commitment. This is not in line with the intrinsic nature of the television medium, which is mainly aimed at entertainment and relaxation.

Interaction with technology seems to generate a cognitive workload in older people, probably due to age-related visual, perceptive, psychomotor, and cognitive disabilities, where too many buttons, many channels, numbers to remember, etc. cause confusion. This often produces cognitive stress with fear, a sense of inadequacy, distance, and suspicion.

When asked what features of the new TV were a surprise, before mentioning the technical characteristics (increase in potential, higher number of channels, better reception), the participants answered: "How much it costs me!"

Clearly, participants are not talking about a simple financial cost, but rather a symbolic cost. This emotional cost is paid by those who have invested a curious interest in new technologies and by those who have tried to stay updated with modern times but have felt a sense of frustration and inadequacy from failing to do so. While all participants perceived the DTT offer to have great potential, they complained about the difficulty of exploiting it, leading them to give up and return to traditional TV with disappointment.

\subsubsection{Use Behavior and Attitude Towards DTT}

In this study, Use Behavior is related to the DTT usage by older adults. 
The change produced by DTT can generate two different types of reactions: acceptance or rejection.

Participants who accepted DTT and the new TV channels available in their daily choices. These participants tend to adopt a stimulating attitude towards technology and often take appropriate expedients to overcome problems. These people are the same using their exploratory skills on the Internet (reading online newspapers or looking for old friends on Facebook).

People who refuse DTT try to eliminate the changes produced by it, for instance, by selecting traditional channels and only viewing at those as if none others exist.

\subsubsection{Performance Expectancy}

Performance Expectancy relates in this case to perceived DTT usefulness.

Some immediate answers of participants during the focus group clearly show that nothing changed or improved after the introduction of DTT.

For the older adults, there is a lack of perceived advantages of DTT compared to traditional television. After some reflection later during the focus group, a few advantages were identified, such as a wider range of programs, available content, better resolution, and image quality.

More than half of those who were present (6 out of 10) claimed to have a PC at home.

Only some people stated to use the Internet routinely to read newspapers, keep in touch with friends on Facebook, watch a favorite TV broadcasts at the most convenient hours, write/receive emails, browse among the photos published by grandchildren on the network, or check their bank accounts.

In this segment, the PC was used mostly for emailing and Facebook as sources of communication and to attain human connection; it is no coincidence that this was especially prevalent among women participants.

The Internet is structural means for contact with the world that this group manifests. Wanting to be part of the modern day world is an indicator of openness towards what we call "new technologies," even in its new digital format, in terms of the Internet, social networks, TV, and Internet broadcasts.

\subsubsection{Facilitating Conditions and Social Support}

The switch-off representing the "point of no return" indicated the milestone where elders want to either establish themselves as a membership in the digitized society or not. The introduction of the DTT represents an event that involves the context of which one is a part of, first, the region then the nation. Therefore, the use of DTT builds an emotional feeling of belonging to society with respect to which, if you are not equipped, you can feel outside.

Participants in the focus group were critical towards the support provided to users during the transition process. Older adults were able to solve problems with DTT with the support provided by relatives and friends.

The implicit request that can be traced in the discursive flow of this group seems to support participants with further resources by simplifying modalities that can guarantee a better fruition.

The improvement proposals put forward by the group in the final phase of the focus group precisely lean towards improving the quality of the DTT offer. 


\section{Discussion}

From Field Study 1, it emerges that technology-resistant older adults are aware of the opportunities that such a new technological system can offer to improve their quality of life. The EasyReach system was overall perceived as useful for social interaction (Performance Expectancy). The pleasantness (Hedonic Motivation) and ease of use (Effort Expectancy) were also carefully examined as potential factors that may affect acceptance.

In accordance with other works [17], the results of this investigation suggest that the use of traditional devices to interact with digital environments can lower the risk of rejection by technology-resistant participants and, especially for aged people, could provide a solution to mitigate social isolation. The use of familiar devices (such as TV sets) in the interaction should improve the acceptance of ICT services by older adults.

By engaging older adults in real-life scenarios that did not require direct interaction, this work shows a large collection of opinions and sentiments about a realistically perceived opportunity. Further investigations are needed to assess direct interactions in and evaluate user acceptance in a real-life environment.

The quantitative exploratory Field Study 2 allowed the authors to investigate factors facilitating or hampering the acceptance of an Electronic Health Record by senior citizens and also to compare the results obtained for the overall sample of citizens and for the group of adults over 55 .

Performance Expectancy and Facilitating Conditions appear to be the most relevant determinants of Behavioral Intention to use the TreC system. Performance Expectancy is connected to the perceived usefulness of the TreC system by senior citizens in daily life, improvements in the health management, and opportunities provided by the system to save time. Facilitating Conditions include the availability of technological resources necessary to use TreC, knowledge (skills) necessary to use TreC, and necessary help/support available when difficulties arise during usage. Minor importance appears to be devoted to Effort Expectancy, reflecting both the ease of learning how to use the system at the beginning and ease of use after the first period. In other studies, an important role was devoted to Effort Expectancy in promoting acceptance, especially in the early stages of introducing the service. The less important role of Effort Expectancy in this study, in comparison with other studies [5], can be ascribed to the characteristics of the sample, which was composed by people skilled in technology use and that utilize Internet services in their daily lives.

Overall, the investigation on acceptance of older adults over 55 years of age showed no big differences between general model variables, including all participants. Performance Expectancy scores and Facilitating Conditions seem to be the most relevant determinants of Behavioral Intention to use the TreC system.

This field study was realized by involving older adults who have a high education (degree or post degree), are skilled in technology use, and employed. Further research should focus on the acceptance of TreC services by older adults who have different characteristics, such as less technological skill and lower education.

The results of the TreC field study suggest that public policies to promote acceptance of innovative e-health solutions by older adults should be mainly focused on:

- Increasing the perceived usefulness of the new solutions (for instance, setting up adequate communication campaigns to promote awareness about services made available by EHR); 
- Providing adequate support for facilitating the utilization of health services (such as with online help, a website, or contact center).

The results of Field Study 3 on the acceptance of digital television by older adults suggest that the behavior of the elderly group is not homogeneous. The transition to DTT induced many different emotional reactions. In one way, the innovation was viewed as a menace or too much of a change in how television is traditionally watched on a daily basis, while another reaction was that the innovation was a desirable change and presented a development opportunity.

Each event, concerning the presentation of the technology, received different emotional constructions by each person who is in the same environment and acts in it. From the introduction of DTT, different groups projected their own desires and fears based on learning and acceptance the new technology. Acceptance attitudes (from participants who decided to explore new channels) or rejection attitudes (from those who tried to reset the change by reprogramming the old channels, acting ignorant towards new channels) were defined by the different and specific emotional representations, or cultures, of the analyzed event. In the moments of intense change, the cultural models were revealed with defined clarity. Reactions to the change towards DTT and the relationship with the new telecommunication technologies depend on, and simultaneously reveal, the different cultures of participants.

It was also found that the fear of learning DTT and new ICT technologies prevails against curiosity, or vice versa, to the extent that these two sub-groups are identified based on the following cultural characteristics:

- Fear: Participants fearful of DTT and new technologies changes exhibit a sense of inadequacy, incompetence, and clumsiness.

- Curiosity: Personal interests and passions of older adults in this subclass, who are unskilled in new technologies, allow them to overcome the psychological and physical barriers due to age. This group can be a powerful basis for word-of-mouth, horizontal peer-to-peer promotion and personalized help to complement the support provided by customized help groups and call centers.

\section{Limitations of the Study}

In the field studies, the cognitive impairment level of the recruited older people was not considered. Therefore, the influence of declining cognition on ICT acceptance by older people should be investigated in future studies.

Another limitation is that the factors affecting older adults to accept various ICT services (such as TV-based technology for social interaction, an Electronic Health Record, and Digital Television) were all analyzed within the same reference framework (Unified Theory of Acceptance of Use of Technology). Also, the users involved in the three studies had different socio-demographic characteristics, while different methods were adopted for data collection (i.e., quantitative or qualitative). Consequently, it is not possible to compare the results obtained in the three field studies; thus, only general considerations can be suggested about ICT acceptance by older adults.

Geographical localization of the three field studies, which were performed in Italy, is another limitation of this study. While the analyzed methods could have potential in many different populations and countries, the obtained the results can only be specified to senior citizens in Italy. 


\section{Conclusions}

In order to identify the main predictors of acceptance of ICT services by older adults, the UTAUT and UTAUT2 models were quantitatively and qualitatively employed in three field studies. Overall, results confirm that the reference model variables represent the key factors for older adults in predicting their viewpoint toward ICT.

Performance Expectancy and Facilitating Conditions seem to be the most relevant factors in all field studies. Also, the relevance of Hedonic Motivation emerged clearly. The role of ease of use seems to be affected by the user profiles, which were based on education level, experience with technology, and employment. For instance, in Field Study 2, Effort Expectancy was found to have less importance, most likely due to the characteristics of participants who were skilled in technology use and utilize daily Internet services.

Another important factor overcoming barriers preventing the acceptance of ICTs by older adults is the use of familiar devices (as TV sets) in the interaction, as suggested by Field Study 1.

From Field Study 3, it was apparent that older adults' acceptance/rejection of ICT services depends on classical socio-demographic variables (age, gender, level of education, number of family members, composition of the household, and geographical area) and on specific "cultures," including attitudes towards technology, lifestyle, and beliefs that individuals share with members of their social context.

\section{Author Contributions}

The authors contributed equally to this work.

\section{Competing Interests}

The authors have declared that no competing interests exist.

\section{References}

1. Loos E, Haddon L, Mante-Meijer E, editors. Generational Use of New Media. Ashgate; 2012

2 ISTAT, Cittadini, imprese e ICT, Report; 2017. Available from: https://www.istat.it/it/files//2017/12/ICT_Anno2017.pdf

3 Gomes G, Duarte C, Coelho J, Matos E. Designing a facebook interface for senior users. The Scientific World J. 2014; 2014: 741567. doi: 10.1155/2014/741567.

4. European Commission. Digital agenda for Europe. Luxembourg: Publications Office of the European Union; 2014.

5. Venkatesh V, Morris MG, Davis GB, Davis FD. User acceptance of information technology: Toward a unified view. MIS Quart. 2003; 27: 425-478.

6. Loos E, Haddon L, Mante-Meijer E, editors. The social dynamics of information and communication technology. Ashgate; 2008.

7. Silverstone R, Haddon L. Design and the domestication of information and communication technologies: Technical change and everyday life. Oxford University Press; 1996.

8. Haddon L. Roger silverstone's legacies: domestication. New Media and Society. 2006; 9: 25-32. 
9. Norman DA. Design of everyday things: revised and expanded. London: MIT Press (UK edition); 2013.

10. Hassenzahl M, Diefenbach S, Goritz, A. Needs, affect, and interactive products - Facets of user experience. Interact Comput. 2010; 22: 353-362.

11. Sapio B, Turk T, Cornacchia M, Papa F, Nicolò E, Livi S. Building scenarios of digital television adoption: a pilot study. Technol Anal Strat Manage. 2010; 22: 43-63.

12. Venkatesh $\mathrm{V}$, Thong J, Xu X. Consumer acceptance and use of information technology: Extending the unified theory of acceptance and use of technology. MIS Quart, 2012; 36: 157178.

13. Papa F, Nicolò E, Livi S, Sapio B, Cornacchia M. Factors affecting the usage of payment services through digital television in Italy. Proceedings of European Interactive TV and Video (EurolTV 2010) Conference; 2010 June 9-11; Tampere Finland.

14. Newell AF, Carmichael A, Gregor P, Alm N. Information technology for cognitive support. In: Jacko JA, editor, The human-computer interaction handbook: fundamentals, evolving technologies and emerging applications. Lawrence Erlbaum Associates, Inc.; 2003. 464-481.

15. Papa F, Cornacchia M, Sapio B, Nicolò E. Engaging technology-resistant older adults: Empirical evidence from an ICT-enabled social environment. Inform Health Soc Care. 2017; 42: 18. doi: 10.3109/17538157.2016.1153477.

16. Kamel Boulos MN, Lou RC, Anastasiou A, Nugent CD, Alexandersson J, Zimmermann G, et al. Connectivity for healthcare and well-being management: examples from six European projects. Int J Env Res Pub Heal. 2009; 6: 1947-1971.

17. Alaoui M, Lewkowicz M. Struggling against social isolation of the elderly - The Design of SmartTV Applications. Proceedings of the 10th International Conference on the Design of Cooperative systems; 2012 May 30-June 1; London: Springer.

18. Correia L, Costa N, Pereira A. Fighting elders' social and technological exclusion: the TV based approach. 5th International Symposium on Ambient Intelligence; 2014 04-06 June; Salamanca. London: Springer.

19. Bisiani R, Merico D, Pinardi S, Dominoni M, Cesta A, Orlandini A, et al. Fostering Social Interaction of Home-Bound Older adults: The EasyReach System. In: Ali M, Bosse K, Hindriks V, Hoogendoorn M, Jonker CM, Treur J, editors. Recent Trends in Applied Artificial Intelligence. Springer; 2013. (33-42).

20. Nielsen J. Seniors as Web Users. Nielsen Norman Group; 2013 May 28. Available from: http://www.nngroup.com/articles/usability-for-senior-citizens/.

21. Nielsen J. Authentic Behavior in User Testing. Nielsen Norman Group; 2005 February 14. Available from: http://www.nngroup.com/articles/authenticbehavior-in-user-testing/.

22. Papa F et al. Detailed Plan for the Pilots. Technical Report. EasyReach Project; 2012; Deliverable D6.1

23. Tang PC, Ash JS, Bates DW, Overhage JM, Sands DZ. Personal health records: Definitions, benefits, and strategies for overcoming barriers to adoption. J Am Med Inform Assoc. 2006; 13: 121-127.

24. Zanutto A. "Two Clicks and I'm in!" Patients as co-actors in managing health data through a personal health record infrastructure. Health Inform J. 2017; doi 10.1177/1460458217712056.

25. Papa F, Zanutto A, Nicolò E, Sapio B. Measuring citizens' acceptance of an electronic health record in Italy. Int J Healthc Technol Manage. 2019 
26. Papa F, Sapio B, Pelagalli M F.User experience of older adults with digital television. Proceedings of the 9th international conference on Interactive television (EurolTV '11); 2011 June 29 -July 1; Lisbon Portugal

27. Papa F. Analisi delle esperienze d'uso (user experience) con la televisione digitale terrestre nei principali Paesi Europei. Technical report. Fondazione Ugo Bordoni; 2010

28. Madden M. Older adults and social media: Social networking use among those ages 50 and older nearly doubled over the past year. Technical report. Pew Research Center; 2010

29. Verdegem P, Hauttekeete L, De Marez L. The analogue switch-off in a cable dominated television landscape. Implications for the transition to digital television in Flanders. Communications, the European Journal of Communication Research. 2009; 34: 87-101.

30. Carli R, Paniccia RM, Lancia F, Pelagalli MF, Nuovi modelli di comunicazione e sviluppo territoriale. Psicologia Clinica. 1997; 2: 41-64.

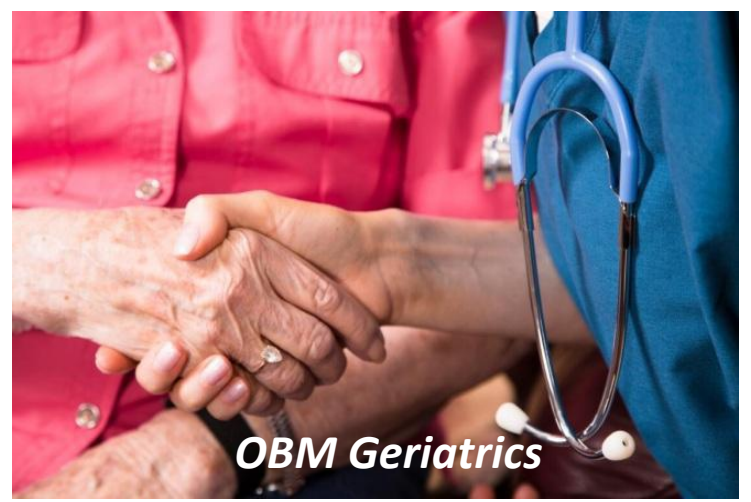

Enjoy OBM Geriatrics by:

5. Submitting a manuscript

6. Joining in volunteer reviewer bank

7. Joining Editorial Board

8. Guest editing a special issue

For more details, please visit: http://www.lidsen.com/journals/geriatrics 
OBM OBM Geriatrics Editorial Office 73 Hongkong Middle Road, Qingdao, China Tel./Fax: +86-532-8979-9572

E-Mail: geriatrics@lidsen.com http://www.lidsen.com/journals/geriatrics

LIDSEN Publishing Inc.

2000 Auburn Drive, One Chagrin Highlands, Suite 200

Beachwood, OH 44122, USA

Tel.: +1-216-370-7293

Fax: +1-216-378-7505

https://www.lidsen.com 
LIDSEN Publishing Inc.

2000 Auburn Drive, One Chagrin

Highlands, Suite 200 Beachwood

$\mathrm{OH} 44122$

USA

Tel.: +1-216-370-7293

Fax: +1-216-378-7505

$\underline{\text { https://www.lidsen.com }}$ 